\title{
Neutron $\beta^{-}-$Decay as Laboratory for Test of Standard Model
}

\author{
A. N. Ivanov, ${ }^{1}$ M. Pitschmann, ${ }^{2,3}$ and N. I. Troitskaya ${ }^{4}$ \\ ${ }^{1}$ Atominstitut, Technische Universität Wien, Stadionalle 2, A-1020 Wien, Austria \\ ${ }^{2}$ University of Wisconsin-Madison, Department of Physics, \\ 1150 University Avenue, Madison, WI 53706, USA \\ ${ }^{3}$ Physics Division, Argonne National Laboratory, Argonne, Illinois 60439, USA \\ 4 State Polytechnic University of St. Petersburg, \\ Polytechnicheskaya 29, 195251, Russian Federation *
}

(Dated: November 6, 2018)

\begin{abstract}
We analyse the sensitivity of all experimentally observable asymmetries and energy distributions for the neutron $\beta^{-}$-decay with a polarized neutron and unpolarised decay proton and electron and the lifetime of the neutron to contributions of order $10^{-4}$ of interactions beyond the Standard model (SM). Since the asymmetries and energy distributions are expressed in terms of the correlation coefficients of the neutron $\beta^{-}$-decay, in order to obtain a theoretical background for the analysis of contributions beyond the SM we revise the calculation of the correlation coefficients within the SM. We take into account a complete set of contributions, induced to next-to-leading order in the large proton mass expansion by the "weak magnetism" and the proton recoil, and the radiative corrections of order $(\alpha / \pi)$, calculated to leading order in the large proton mass expansion. We confirm the results, obtained in literature. The contributions of interactions beyond the SM we analyse in the linear approximation with respect to the Herczeg phenomenological coupling constants, introduced at the hadronic level. Such an approximation is good enough for the analysis of contributions of order $10^{-4}$ of interactions beyond the SM. We show that in such an approximation the correlation coefficients depend only on the axial coupling constant, which absorbs the contributions of the Herczeg left-left and left-right lepton-nucleon current-current interactions (vector and axial-vector interactions beyond the SM), and the Herczeg scalar and tensor coupling constants. In the lifetime of the neutron in addition to the axial coupling constant the contributions of the Herczeg left-left and left-right lepton-nucleon current-current interactions (vector and axial-vector interactions beyond the SM) are absorbed by the Cabibbo-Kobayashi-Maskawa (CKM) matrix element.
\end{abstract}

PACS numbers: 12.15.Ff, 13.15.+g, 23.40.Bw, 23.50.+z

\section{INTRODUCTION}

In this paper we propose a consistent analysis of the sensitivity of all observable asymmetries and energy distributions of the neutron $\beta^{-}$-decay $n \rightarrow p+e^{-}+\bar{\nu}_{e}$ with a polarized neutron and unpolarised decay proton and electron and the lifetime of the neutron [1, 2] to contributions of order $10^{-4}$ of interactions beyond the Standard model (SM), described at the phenomenological level. Such an order of corrections beyond the SM has been pointed out by Ramsey-Musolf and Su 3] within the Minimal Supersymmetric extension of the SM (MSSM). As has been shown in [4]-7], at the phenomenological level the neutron $\beta^{-}$-decay may be described by eight complex coupling constants determining the strength of interactions beyond the SM. As a result possible deviations from the SM, causing the contributions of order $10^{-4}$ to the correlation coefficients of the neutron $\beta^{-}$-decay and the lifetime of the neutron, may be determined in terms of vector, axial-vector, scalar and tensor lepton-baryon weak coupling constants 8].

It is well-known [1, 2] that in the non-relativistic approximation to leading order in the large proton mass expansion, which is equivalent to the leading order of the heavy-baryon approximation, and in the rest frame of the neutron the SM with weak $V-A$ interactions [9] describes the $\beta^{-}$-decay of the neutron in terms of two coupling constants $G_{V}$ and $G_{A}$ [1, 2] (see also 10] ). The coupling constant $G_{V}$ is defined by the product $G_{V}=G_{F} V_{u d}$ of the Fermi coupling constant $G_{F}=g_{W}^{2} / 8 M_{W}^{2}[9]$, where $g_{W}$ and $M_{W}$ are the electroweak coupling constant and the $W$-boson mass, and $V_{u d}$ is the matrix element of the Cabibbo-Kobayashi-Maskawa (CKM) quark mixing matrix [9]. The coupling constant $G_{A}$ is equal to $G_{A}=\lambda G_{V}$, where $\lambda=G_{A} / G_{V}$ is the axial coupling constant, induced by renormalisation

*Electronic address: ivanov@kph.tuwien.ac.at 
of the axial hadronic current by strong low-energy interactions [11]. For the weak interactions, invariant under time reversal, the coupling constant $\lambda$ is real.

The observables of the neutron $\beta^{-}$-decay with unpolarised particles are the lifetime of the neutron $\tau_{n}$ and the correlation coefficient $a_{0}$, describing correlations between 3-momenta of the electron and antineutrino to leading order in the large proton mass expansion in the rest frame of the neutron. Experimentally the correlation coefficient $a_{0}$ can be determined, for example, by measuring either the electron-proton energy distribution $a\left(E_{e}, T_{p}\right)$, where $E_{e}$ and $T_{p}$ are the total and kinetic energies of the electron and proton, respectively, or the proton-energy spectrum $a\left(T_{p}\right)$. As a function of the electron energy $E_{e}$ the correlation coefficient of correlations between the electron and antineutrino $3-$ momenta we denote as $a\left(E_{e}\right)$.

The neutron $\beta^{-}$-decay with a polarized neutron and unpolarised decay proton and electron is characterized also by two additional observable correlation coefficients $A_{0}$ and $B_{0}$, describing to leading order in the large proton mass expansion correlations between the neutron spin and 3-momenta of the electron and antineutrino, respectively. As functions of the electron energy $E_{e}$ the correlation coefficients of correlations between the neutron spin and 3momenta of the electron and antineutrino we denote as $A\left(E_{e}\right)$ and $B\left(E_{e}\right)$, respectively. The lifetime of the neutron and the correlation coefficients under consideration are of order $\tau_{n} \sim 880 \mathrm{~s}, a_{0} \sim a\left(E_{e}\right) \sim A_{0} \sim A\left(E_{e}\right) \sim-0.1$ and $B_{0} \sim B\left(E_{e}\right) \sim 1[9]$. The coupling constants $G_{V}$ and $\lambda$ define the main contributions to the lifetime of the neutron and the correlation coefficients [1, 2] (see also [10]).

However for the description of the neutron $\beta^{-}$-decay at the modern level of experimental accuracies when the experimental data on the axial coupling constant $\lambda=-1.2750(9)$ [1] and the lifetime of the neutron $\tau_{n}^{(\exp )}=878.5(8) \mathrm{s}$ 12] have been obtained with accuracies $0.07 \%$ and $0.09 \%$, respectively, two coupling constants $G_{V}$ and $\lambda$ are not enough for the correct description of the properties of the neutron $\beta^{-}$-decay. Indeed, it is well-known that the radiative corrections to the lifetime of the neutron [13]-[30], calculated to leading order in the large proton mass expansion within the SM with the $V-A$ weak interactions and Quantum Electrodynamics (QED), give the contribution of about $3.9 \%$ 28] (see also [1]). This allows to describe correctly the lifetime of the neutron [10]. The radiative corrections are very important also for the correct determination of the Fermi coupling constant $G_{F}$. It may be extracted from the experimental data on the weak coupling constant $G_{\mu}$ of the $\mu^{-}$-decay $\mu^{-} \rightarrow e^{-}+\nu_{\mu}+\bar{\nu}_{e}$ with the account for the radiative corrections [13]-[16]. The contributions of the radiative corrections to the ratios of the correlation coefficients $a\left(E_{e}\right) / a_{0}$ and $A\left(E_{e}\right) / A_{0}$ are by order of magnitude smaller compared with the contribution to the lifetime of the neutron, whereas the correlation coefficient $B\left(E_{e}\right)$ has no radiative corrections to order $\alpha / \pi[31$, 32].

Another type of corrections, which may be calculated within the SM and should be taken into account for the description of the neutron $\beta^{-}$-decay on the same footing as the radiative corrections, are the contributions of the "weak magnetism" and the proton recoil [33, 34] (see also [32]), calculated to next-to-leading order in the large proton mass expansion.

The radiative corrections and the corrections from the "weak magnetism" and the proton recoil, calculated to leading order and to next-to-leading order in the large proton mass expansion, respectively, define a complete set of corrections to the observables of the neutron $\beta^{-}$-decay, which should be taken into account within the SM as a background for the analysis of contributions of order $10^{-4}$ of interactions beyond the SM.

Since the observable asymmetries and energy distributions are defined in terms of the correlation coefficients [1], for the aim of the paper to analyse the sensitivity of the observables of the neutron $\beta^{-}$-decay to contributions of order $10^{-4}$ of interactions beyond the SM we revise the calculation of the correlation coefficients of the neutron $\beta^{-}-$decay with a polarized neutron and unpolarised decay proton and electron and the lifetime of the neutron within the SM with $V-A$ weak interactions. In addition to the non-relativistic terms $a_{0}, A_{0}$ and $B_{0}$, calculated to leading order in the large proton mass expansion, we take into account 1) the contributions of the "weak magnetism" and the proton recoil to next-to-leading order in the large $M$ expansion, which provide a complete set of corrections of order $1 / M$, where $M$ is an average mass of the neutron and proton $M=\left(m_{n}+m_{p}\right) / 2$, and 2) the radiative corrections of order $\alpha / \pi$, calculated to leading order in the large $M$ expansion, where $\alpha=1 / 137.036$ is the fine-structure constant [9]. The small parameter of the large $M$ expansion is $E_{0} / M \sim 10^{-3}$, where $E_{0} \sim 1 \mathrm{MeV}$ is the end-point energy of the electron-energy spectrum. The parameter $E_{0} / M \sim 10^{-3}$ is commensurable with the parameter of the radiative corrections $\alpha / \pi \sim 10^{-3}$. As we show below due to the strong dependence on the axial coupling constant $\lambda$ the numerical values of the $1 / M$ corrections vary from $10^{-5}$ to $10^{-1}$. In turn the contributions of the radiative corrections do not depend on the axial coupling constant $\lambda$. Following Sirlin [18] we show that the contributions of the radiative corrections, depending on the axial coupling constant $\lambda$, may be absorbed by renormalisation of the Fermi $G_{F}$ and axial $\lambda$ coupling constants. The contributions of radiative corrections to the ratios $a\left(E_{e}\right) / a_{0}$ and $A\left(E_{e}\right) / A_{0}$ are of order $10^{-3}$. The correlation coefficients, calculated within the SM to order $1 / M$ and $\alpha / \pi$, determine the theoretical background for the analysis of contributions of order $10^{-4}$ beyond the SM.

A phenomenological analysis of contributions of interactions beyond the SM model shows that these interactions induce only 1) the energy independent contributions and 2) the contributions proportional to $m_{e} / E_{e}$, where $m_{e}$ is the electron mass. We show below that the contributions of the "weak magnetism" and the proton recoil into the terms 
proportional to $m_{e} / E_{e}$ of the correlation coefficients $a\left(E_{e}\right), A\left(E_{e}\right)$ and $B\left(E_{e}\right)$ are of order $10^{-3}-10^{-4}$. Thus, the obtained theoretical expressions for the correlation coefficients should be taken into account for a correct experimental determination of contributions of order $10^{-4}$ beyond the SM.

The radiative corrections to the correlation coefficients $a\left(E_{e}\right) / a_{0}$ and $A\left(E_{e}\right) / A_{0}$ determine the most important and complicated part of the corrections of order $10^{-3}$. In the neutron $\beta^{-}$-decay the radiative corrections are defined by 1 ) the contributions to the continuum-state $\beta^{-}$-decay mode from one-virtual photon exchanges, $W$-boson and $Z$-boson exchanges and QCD corrections 24, 27]-30] and 2) the contribution of the radiative $\beta^{-}-$decay mode $n \rightarrow p+e^{-}+\bar{\nu}_{e}+\gamma$ with emission of a real photon [13]-30] (see also [31, 32]). The sum of the electron-energy and angular distributions of these two decay modes does not suffer from infrared divergences of virtual and real photons.

The radiative corrections to the $\beta^{-}$-decay of the neutron we calculate within the standard finite-photon mass (FPM) regularization of infrared divergences [13] - 30]. As has been shown by Marciano and Sirlin [22], the FPM regularization is equivalent to the dimensional regularization [35].

The radiative corrections to the lifetime of the neutron, calculated within the FPM regularization, obey the Kinoshita-Lee-Nauenberg (KLN) theorem [36]. According to the KLN theorem, the radiative corrections to the lifetime of the neutron, integrated over the phase volume of the final state of the neutron $\beta^{-}-$decay in the limit of the massless electron $m_{e} \rightarrow 0$, should not depend on the electron mass $m_{e}$. For the first time such an independence of the electron mass in the limit $m_{e} \rightarrow 0$ has been demonstrated by Kinoshita and Sirlin [15].

We reproduce fully the radiative corrections to the lifetime of the neutron and the correlation coefficients $a\left(E_{e}\right)$ and $A\left(E_{e}\right)$, calculated in [16]-30] and [31, 32], respectively (see Eq.(17) and Appendix D). We show also that the correlation coefficient $B\left(E_{e}\right)$ has no radiative corrections to order $\alpha / \pi$, calculated to leading order in the large $M$ expansion. This agrees well with the results, obtained by Gudkov et al. 32]. The corrections of the "weak magnetism" and the proton recoil we calculate in complete agreement with the results, obtained in [32] (see also [34]). Above the background of these corrections the contributions of interactions beyond the SM are calculated to leading order in the large $M$ expansion and in the linear approximation with respect to the Herczeg phenomenological coupling constants, introduced at the hadronic level in terms of the lepton-nucleon current-current interactions (see Appendix G). Such an approximation is good enough for the analysis of the sensitivity of the observables of the neutron $\beta^{-}-$decay to contributions of order $10^{-4}$ beyond the SM.

The obtained results are applied to the theoretical analysis of 1$)$ the asymmetries $A_{\exp }\left(E_{e}\right), B_{\exp }\left(E_{e}\right)$, the electronproton energy distribution $a\left(E_{e}, T_{p}\right)$, the proton-energy spectrum $a\left(T_{p}\right)$ and the proton recoil asymmetry $C_{\text {exp }}$, used for the experimental determination of the axial coupling constant $\lambda$ and the correlation coefficients $A_{0}, B_{0}, a_{0}$ and $C_{0}=-x_{C}\left(A_{0}+B_{0}\right)$, where $x_{C}=0.27591$ is a theoretical numerical factor (see section VII), respectively, 2) the lifetime of the neutron and 3) the sensitivity of the electron-proton energy distribution $a\left(E_{e}, T_{p}\right)$, the proton-energy spectrum $a\left(T_{p}\right)$, the asymmetries $A_{\exp }\left(E_{e}\right), B_{\exp }\left(E_{e}\right)$ and $C_{\exp }$ and the lifetime of the neutron $\tau_{n}$ to contributions of order $10^{-4}$ of interactions beyond the SM. The experimental analysis of the asymmetries $A_{\exp }\left(E_{e}\right), B_{\exp }\left(E_{e}\right)$, the proton-energy spectrum $a\left(T_{p}\right)$ and the proton recoil asymmetry $C_{\exp }$ has been carried out in [1, 37], [38, 39], [40] (see also [41]) and [42]), respectively.

\section{ORGANIZATION OF PAPER}

The paper is organized as follows. In section III we calculate the electron-energy and angular distribution of the neutron $\beta^{-}$-decay with a polarized neutron and unpolarised decay proton and electron. We take into account a complete set of corrections of order $1 / M$, caused by the "weak magnetism" and the proton recoil, and the radiative corrections of order $\alpha / \pi$. Then we analyse 1$)$ in section IV the electron asymmetry $A_{\exp }\left(E_{e}\right)$, which has been used in [1, 37] for the experimental determination of the axial coupling constant $\lambda=-1.2750(9)$ and the correlation coefficient $\left.A_{0}^{(\exp )}=-0.11933(34), 2\right)$ in section $\mathrm{V}$ the antineutrino asymmetry $B_{\exp }\left(E_{e}\right)$, which has been used for the experimental determination of the correlation coefficient $B_{0}^{(\exp )}=0.9802(50)$ in 38 , 39], 3) in section VI the electron-proton energy distribution $a\left(E_{e}, T_{p}\right)$ and the proton-energy spectrum $a\left(T_{p}\right)$, which may be used for the experimental determination of the axial coupling constant $\lambda$ and the correlation coefficient $a_{0}$, 4) in section VII the proton recoil asymmetry $C_{\text {exp }}$, which has been used for the experimental determination of the correlation coefficient $C_{0}^{(\exp )}=-x_{C}\left(A_{0}+B_{0}\right)=-0.2377(26)$ [42], and 5) in section VIII the lifetime of the neutron. In section IX] we propose the theoretical analysis of the sensitivity of the electron-proton energy distribution $a\left(E_{e}, T_{p}\right)$, the protonenergy spectrum $a\left(T_{p}\right)$, the asymmetries $A_{\exp }\left(E_{e}\right), B_{\exp }\left(E_{e}\right)$ and $C_{\exp }$ and the lifetime of the neutron to contributions of order $10^{-4}$ of interactions beyond the SM. We show that to linear approximation with respect to the Herczeg phenomenological coupling constants the Herczeg coupling constants $a_{L L}^{h}$ and $a_{L R}^{h}$ of the left-left and left-right leptonnucleon current-current interactions (vector and axial-vector interactions beyond the SM) can be fully absorbed by the axial coupling constants and cannot be determined from the experimental data on the electron-proton energy 
distribution $a\left(E_{e}, T_{p}\right)$, the proton-energy spectrum $a\left(T_{p}\right)$ and the asymmetries $A_{\exp }\left(E_{e}\right), B_{\exp }\left(E_{e}\right)$ and $C_{\exp }\left(E_{e}\right)$. This agrees well with the results obtained in [43]-[45]. In the lifetime of the neutron the contributions of the Herczeg left-left and left-right lepton-nucleon current-current interactions (vector and axial-vector interactions beyond the $\mathrm{SM}$ ) become unobservable after the renormalisation of the CKM matrix element $V_{u d} \rightarrow\left(V_{u d}\right)_{\mathrm{eff}}=V_{u d}\left(1+a_{L L}^{h}+a_{L R}^{h}\right)$ (see section [X] in agreement with [43]- 45]. In the conclusion $\mathrm{X}$ we summarized the obtained results. In Appendix A we give a detailed calculation of the amplitude, the electron-energy and angular distribution of the continuumstate $\beta^{-}$-decay of the neutron to next-to-leading order in the large $M$ expansion. In Appendix B we calculate the rate, the electron-energy and angular distribution of the radiative $\beta^{-}$-decay of the neutron with a polarized neutron and unpolarised decay particles and their contributions to the rate, the electron-energy and angular distribution of the neutron $\beta^{-}$-decay. We compare our results for the branching ratios of the radiative $\beta^{-}-$decay of the neutron with the experimental data, obtained by Nico et al. [46] and Cooper et al. 47] (see also [48]), and the theoretical results, obtained in [49, 50]. In Appendix C within the FPM regularization [13] - 30] we analyse the infrared divergent contributions of one-virtual photon exchanges to the continuum-state $\beta^{-}$-decay mode of the neutron. In Appendix $\mathrm{D}$ we give a detailed calculation of the radiative corrections, caused by one-virtual photon exchanges in the continuumstate $\beta$-decay of the neutron and the radiative $\beta^{-}$-decay of the neutron. We define the radiative corrections to the lifetime of the neutron and the correlation coefficients by the functions $g_{n}\left(E_{e}\right)$ and $f_{n}\left(E_{e}\right)$, respectively. We show also that in the rest frame of the neutron and in the non-relativistic approximation for the proton one--virtual photon exchanges induce effective scalar and tensor weak lepton-baryon couplings of order $\alpha G_{F} / \pi$ and $\alpha \lambda G_{F} / \pi$, respectively, depending on the electron energy $E_{e}$. In Appendix $\mathrm{E}$ we show that the radiative corrections to the lifetime of the neutron, defined by the function $g_{n}\left(E_{e}\right)$, satisfy the KLN theorem. This agrees with the analysis of the radiative corrections, carried out in [15]. In Appendix F we calculate the radiative corrections to the lifetime of the neutron, using the procedure proposed by Sirlin [18]. We confirm that unambiguity of observable radiative corrections to the lifetime of the neutron is caused by gauge invariance of the amplitude of one-virtual photon exchanges in the continuum-state $\beta^{-}$-decay of the neutron [18]. In Appendix $\mathrm{G}$ we calculate the contributions to the correlation coefficients and the lifetime of the neutron from phenomenological vector, axial-vector, scalar and tensor interactions beyond the SM [4] - 7]. In Appendix H we calculate the proton recoil corrections, caused by the electronproton Coulomb interaction in the final state of the neutron $\beta^{-}$-decay. We show that in the experimentally used electron energy region $250 \mathrm{keV} \leq T_{e}=E_{e}-m_{e} \leq 455 \mathrm{keV}[1]$ the contributions of these corrections to the correlation coefficients are of order $10^{-6}-10^{-5}$ and may be neglected for the analysis of contributions of order $10^{-4}$. In Appendix I we give a detailed calculation of the electron-proton energy-momentum and angular distribution of the neutron $\beta^{-}$-decay with a polarized neutron and unpolarised decay proton and electron. We calculate the electron-proton energy distribution $a\left(E_{e}, T_{p}\right)$, the proton-energy spectrum $a\left(T_{p}\right)$ and the correlation coefficient $C$ of the proton recoil asymmetry by taking into account the $1 / M$ corrections from the "weak magnetism" and the proton recoil and the radiative corrections of order $\alpha / \pi$, calculated to leading order in the large $M$ expansion.

\section{ELECTRON-ENERGY AND ANGULAR DISTRIBUTION OF NEUTRON $\beta^{-}-$DECAY IN STANDARD MODEL}

For the analysis of the electron-energy and angular distribution of the continuum-state $\beta^{-}$-decay of the neutron we use the Hamiltonian of $V-A$ interactions with a real axial coupling constant $\lambda$ and the contribution of the "weak magnetism" 35,51$]$

$$
\mathcal{H}_{W}(x)=\frac{G_{F}}{\sqrt{2}} V_{u d}\left\{\left[\bar{\psi}_{p}(x) \gamma_{\mu}\left(1+\lambda \gamma^{5}\right) \psi_{n}(x)\right]+\frac{\kappa}{2 M} \partial^{\nu}\left[\bar{\psi}_{p}(x) \sigma_{\mu \nu} \psi_{n}(x)\right]\right\}\left[\bar{\psi}_{e}(x) \gamma^{\mu}\left(1-\gamma^{5}\right) \psi_{\nu}(x)\right]
$$

invariant under time reversal, where $\psi_{p}(x), \psi_{n}(x), \psi_{e}(x)$ and $\psi_{\nu}(x)$ are the field operators of the proton, neutron, electron and antineutrino, respectively, $\gamma^{\mu}, \gamma^{5}$ and $\sigma^{\mu \nu}=\frac{i}{2}\left(\gamma^{\mu} \gamma^{\nu}-\gamma^{\nu} \gamma^{\mu}\right)$ are the Dirac matrices [35] and $\kappa=$ $\kappa_{p}-\kappa_{n}=3.7058$ is the isovector anomalous magnetic moment of the nucleon, defined by the anomalous magnetic moments of the proton $\kappa_{p}=1.7928$ and the neutron $\kappa_{n}=-1.9130$ and measured in nuclear magneton [9].

For numerical calculations we use $G_{F}=1.1664 \times 10^{-11} \mathrm{MeV}^{-2}$ and $\left|V_{u d}\right|=0.97427(15)$ [9]. The value of the CKM matrix element $\left|V_{u d}\right|=0.97427(15)$ agrees well with $\left|V_{u d}\right|=0.97425(22)$, measured from the superallowed $0^{+} \rightarrow 0^{+}$ nuclear $\beta^{-}$-decays [52]. It satisfies also well the unitarity condition $\left|V_{u d}\right|^{2}+\left|V_{u s}\right|^{2}+\left|V_{u b}\right|^{2}=0.99999(41)$ for the CKM matrix elements [9]. The error $\Delta_{\mathrm{U}}= \pm 0.00041$ of the unitarity condition is determined by the errors of the CKM matrix elements $\left|V_{u d}\right|=0,97427 \pm 0.00015,\left|V_{u s}\right|=0.22534 \pm 0.00065$ and $\left|V_{u b}\right|=0.00351_{-0.00014}^{+0.00015}$ (see Eq.(11.27) in p. 162 of Ref. [9] ). As a result the error of the unitarity condition is equal to

$$
\Delta_{\mathrm{U}}=\sqrt{\sum_{q}\left|2 V_{u q} \Delta V_{u q}\right|^{2}}=0.00041,
$$


where $q=d, s, b$ and $\Delta V_{u d}=0.00015, \Delta V_{u s}=0.00065$ and $\Delta V_{u b}=0.00015$, respectively.

The amplitude of the continuum-state $\beta^{-}$-decay of the neutron, calculated in the rest frame of the neutron and to next-to-leading order in the large $M$ expansion taking into account the contributions of the "weak magnetism" and the proton recoil, is (see Appendix A)

$$
\begin{aligned}
& M\left(n \rightarrow p e^{-} \bar{\nu}_{e}\right)=-2 m_{n} \frac{G_{F}}{\sqrt{2}} V_{u d}\left\{\left[\varphi_{p}^{\dagger} \varphi_{n}\right]\left[\bar{u}_{e} \gamma^{0}\left(1-\gamma^{5}\right) v_{\bar{\nu}}\right]-\tilde{\lambda}\left[\varphi_{p}^{\dagger} \vec{\sigma} \varphi_{n}\right] \cdot\left[\bar{u}_{e} \vec{\gamma}\left(1-\gamma^{5}\right) v_{\bar{\nu}}\right]\right. \\
& \left.-\frac{m_{e}}{2 M}\left[\varphi_{p}^{\dagger} \varphi_{n}\right]\left[\bar{u}_{e}\left(1-\gamma^{5}\right) v_{\bar{\nu}}\right]+\frac{\tilde{\lambda}}{2 M}\left[\varphi_{p}^{\dagger}\left(\vec{\sigma} \cdot \vec{k}_{p}\right) \varphi_{n}\right]\left[\bar{u}_{e} \gamma^{0}\left(1-\gamma^{5}\right) v_{\bar{\nu}}\right]-i \frac{\kappa+1}{2 M}\left[\varphi_{p}^{\dagger}\left(\vec{\sigma} \times \vec{k}_{p}\right) \varphi_{n}\right] \cdot\left[\bar{u}_{e} \vec{\gamma}\left(1-\gamma^{5}\right) v_{\bar{\nu}}\right]\right\},
\end{aligned}
$$

where $\varphi_{p}$ and $\varphi_{n}$ are the Pauli spinorial wave functions of the proton and neutron and $u_{e}$ and $v_{\bar{\nu}}$ are the Dirac bispinor wave functions of the electron and antineutrino, respectively. Then, $\vec{k}_{p}$ is a $3-$ momentum of the proton related to 3-momenta of the electron $\vec{k}_{e}$ and antineutrino $\vec{k}$ as $\vec{k}_{p}=-\vec{k}_{e}-\vec{k}, \tilde{\lambda}=\lambda\left(1-E_{0} / 2 M\right)$, where $E_{0}=\left(m_{n}^{2}-m_{p}^{2}+\right.$ $\left.m_{e}^{2}\right) / 2 m_{n}=1.2927 \mathrm{MeV}$ is the end-point energy of the electron-energy spectrum, calculated for $m_{n}=939.5654 \mathrm{MeV}$, $m_{p}=938.2720 \mathrm{MeV}$ and $m_{e}=0.5110 \mathrm{MeV}$ [9]. From Eq. (2) one may see that the parameter of the large $M$ expansion or the $1 / M$ corrections to the amplitude of the $\beta^{-}$-decay of the neutron is $k_{p} / M \sim E_{0} / M \sim 10^{-3}$. The detailed calculation of the amplitude Eq.(2) is given in Appendix A.

The electron-energy and angular distribution of the neutron $\beta^{-}$-decay takes the form [1, 32.

$$
\begin{aligned}
& \frac{d^{5} \lambda_{n}\left(E_{e}, \vec{k}_{e}, \vec{k}, \vec{\xi}_{n}\right)}{d E_{e} d \Omega_{e} d \Omega}=\left(1+3 \lambda^{2}\right) \frac{G_{F}^{2}\left|V_{u d}\right|^{2}}{32 \pi^{5}}\left(E_{0}-E_{e}\right)^{2} \sqrt{E_{e}^{2}-m_{e}^{2}} E_{e} F\left(E_{e}, Z=1\right) \Phi_{\beta_{c}^{-}}\left(\vec{k}_{e}, \vec{k}\right) \tilde{\zeta}\left(E_{e}\right) \\
& \times\left(1+\tilde{a}\left(E_{e}\right) \frac{\vec{k}_{e} \cdot \vec{k}}{E_{e} E}+\tilde{A}\left(E_{e}\right) \frac{\vec{\xi}_{n} \cdot \vec{k}_{e}}{E_{e}}+\tilde{B}\left(E_{e}\right) \frac{\vec{\xi}_{n} \cdot \vec{k}}{E}+\tilde{K}_{n}\left(E_{e}\right) \frac{\left(\vec{\xi}_{n} \cdot \vec{k}_{e}\right)\left(\vec{k}_{e} \cdot \vec{k}\right)}{E_{e}^{2} E}+\tilde{Q}_{n}\left(E_{e}\right) \frac{\left(\vec{\xi}_{n} \cdot \vec{k}\right)\left(\vec{k}_{e} \cdot \vec{k}\right)}{E_{e} E^{2}}\right. \\
& \left.+\tilde{D}\left(E_{e}\right) \frac{\vec{\xi}_{n} \cdot\left(\vec{k}_{e} \times \vec{k}\right)}{E_{e} E}\right)
\end{aligned}
$$

where $E=E_{0}-E_{e}$ is the antineutrino energy and $d \Omega_{e}$ and $d \Omega$ are the infinitesimal elements of the solid angles of the electron and antineutrino 3-momenta relative to the neutron spin, respectively.

The function $\Phi_{\beta_{c}^{-}}\left(\vec{k}_{e}, \vec{k}\right)$ is defined by the contribution of the proton recoil. To next-to-leading order in the large $M$ expansion it is equal to (see Eqs. A-19 - A-23 in Appendix A)

$$
\Phi_{\beta_{c}^{-}}\left(\vec{k}_{e}, \vec{k}\right)=1+\frac{3}{M}\left(E_{e}-\frac{\vec{k}_{e} \cdot \vec{k}}{E}\right) .
$$

The function $F\left(E_{e}, Z=1\right)$ is the relativistic Fermi function [59, 60] (see also [34])

$$
F\left(E_{e}, Z=1\right)=\left(1+\frac{1}{2} \gamma\right) \frac{4\left(2 r_{p} m_{e} \beta\right)^{2 \gamma}}{\Gamma^{2}(3+2 \gamma)} \frac{e^{\pi \alpha / \beta}}{\left(1-\beta^{2}\right)^{\gamma}}\left|\Gamma\left(1+\gamma+i \frac{\alpha}{\beta}\right)\right|^{2},
$$

where $\beta=k_{e} / E_{e}=\sqrt{E_{e}^{2}-m_{e}^{2}} / E_{e}$ is the electron velocity, $\gamma=\sqrt{1-\alpha^{2}}-1, r_{p}$ is the electric radius of the proton and $\alpha=1 / 137.036$ is the fine-structure constant. In numerical calculations we will use $r_{p}=0.841 \mathrm{fm}[53$ ]

Following [32] we transcribe the r.h.s. of Eq.(3) into the form

$$
\begin{aligned}
& \frac{d^{5} \lambda_{n}\left(E_{e}, \vec{k}_{e}, \vec{k}, \vec{\xi}_{n}\right)}{d E_{e} d \Omega_{e} d \Omega}=\left(1+3 \lambda^{2}\right) \frac{G_{F}^{2}\left|V_{u d}\right|^{2}}{32 \pi^{5}}\left(E_{0}-E_{e}\right)^{2} \sqrt{E_{e}^{2}-m_{e}^{2}} E_{e} F\left(E_{e}, Z=1\right) \zeta\left(E_{e}\right) \\
& \times\left\{1+a\left(E_{e}\right) \frac{\vec{k}_{e} \cdot \vec{k}}{E_{e} E}+A\left(E_{e}\right) \frac{\vec{\xi}_{n} \cdot \vec{k}_{e}}{E_{e}}+B\left(E_{e}\right) \frac{\vec{\xi}_{n} \cdot \vec{k}}{E}+K_{n}\left(E_{e}\right) \frac{\left(\vec{\xi}_{n} \cdot \vec{k}_{e}\right)\left(\vec{k}_{e} \cdot \vec{k}\right)}{E_{e}^{2} E}+Q_{n}\left(E_{e}\right) \frac{\left(\vec{\xi}_{n} \cdot \vec{k}\right)\left(\vec{k}_{e} \cdot \vec{k}\right)}{E_{e} E^{2}}\right. \\
& \left.+D\left(E_{e}\right) \frac{\vec{\xi}_{n} \cdot\left(\vec{k}_{e} \times \vec{k}\right)}{E_{e} E}-3 \frac{E_{e}}{M} \frac{1-\lambda^{2}}{1+3 \lambda^{2}}\left(\frac{\left(\vec{k}_{e} \cdot \vec{k}\right)^{2}}{E_{e}^{2} E^{2}}-\frac{1}{3} \frac{k_{e}^{2}}{E_{e}^{2}}\right)\right\} .
\end{aligned}
$$



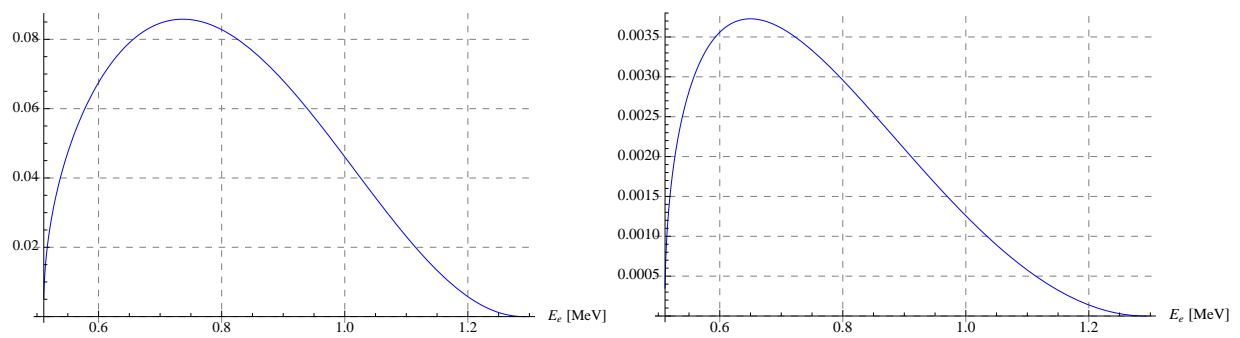

FIG. 1: The densities $(\alpha / \pi) g_{n}\left(E_{e}\right) \rho_{\beta_{c}^{-}}\left(E_{e}\right)$ (left) and $(\alpha / \pi) f_{n}\left(E_{e}\right) \rho_{\beta_{c}^{-}}\left(E_{e}\right)$ (right), measured in $\mathrm{MeV}^{-1}$, of the radiative corrections to the lifetime of the neutron and the correlation coefficients $a\left(E_{e}\right)$ and $A\left(E_{e}\right)$, where $\rho_{\beta_{c}^{-}}\left(E_{e}\right)$ is the electronenergy spectrum density Eq. (D-59).

The correlation coefficients are given by (see Appendix A, B, C and D)

$$
\begin{aligned}
& \zeta\left(E_{e}\right)=\left(1+\frac{\alpha}{\pi} g_{n}\left(E_{e}\right)\right)+\frac{1}{M} \frac{1}{1+3 \lambda^{2}}\left[-2 \lambda(\lambda-(\kappa+1)) E_{0}+\left(10 \lambda^{2}-4(\kappa+1) \lambda+2\right) E_{e}\right. \\
& \left.-2 \lambda(\lambda-(\kappa+1)) \frac{m_{e}^{2}}{E_{e}}\right] \\
& \zeta\left(E_{e}\right) a\left(E_{e}\right)=a_{0}\left(1+\frac{\alpha}{\pi} g_{n}\left(E_{e}\right)+\frac{\alpha}{\pi} f_{n}\left(E_{e}\right)\right)+\frac{1}{M} \frac{1}{1+3 \lambda^{2}}\left[2 \lambda(\lambda-(\kappa+1)) E_{0}-4 \lambda(3 \lambda-(\kappa+1)) E_{e}\right], \\
& \zeta\left(E_{e}\right) A\left(E_{e}\right)=A_{0}\left(1+\frac{\alpha}{\pi} g_{n}\left(E_{e}\right)+\frac{\alpha}{\pi} f_{n}\left(E_{e}\right)\right)+\frac{1}{M} \frac{1}{1+3 \lambda^{2}}\left[\left(\lambda^{2}-\kappa \lambda-(\kappa+1)\right) E_{0}\right. \\
& \left.-\left(5 \lambda^{2}-(3 \kappa-4) \lambda-(\kappa+1)\right) E_{e}\right], \\
& \zeta\left(E_{e}\right) B\left(E_{e}\right)=B_{0}\left(1+\frac{\alpha}{\pi} g_{n}\left(E_{e}\right)\right)+\frac{1}{M} \frac{1}{1+3 \lambda^{2}}\left[-2 \lambda(\lambda-(\kappa+1)) E_{0}\right. \\
& \left.+\left(7 \lambda^{2}-(3 \kappa+8) \lambda+(\kappa+1)\right) E_{e}-\left(\lambda^{2}-(\kappa+2) \lambda+(\kappa+1)\right) \frac{m_{e}^{2}}{E_{e}}\right] \\
& \zeta\left(E_{e}\right) K_{n}\left(E_{e}\right)=\frac{1}{M} \frac{1}{1+3 \lambda^{2}}\left(5 \lambda^{2}-(\kappa-4) \lambda-(\kappa+1)\right) E_{e}, \\
& \zeta\left(E_{e}\right) Q_{n}\left(E_{e}\right)=\frac{1}{M} \frac{1}{1+3 \lambda^{2}}\left[\left(\lambda^{2}-(\kappa+2) \lambda+(\kappa+1)\right) E_{0}-\left(7 \lambda^{2}-(\kappa+8) \lambda+(\kappa+1)\right) E_{e}\right], \\
& \zeta\left(E_{e}\right) D\left(E_{e}\right)=0,
\end{aligned}
$$

where the correlation coefficients $a_{0}, A_{0}$ and $B_{0}$ are determined by [1] (see also [10])

$$
a_{0}=\frac{1-\lambda^{2}}{1+3 \lambda^{2}} \quad, \quad A_{0}=-2 \frac{\lambda(1+\lambda)}{1+3 \lambda^{2}} \quad, \quad B_{0}=-2 \frac{\lambda(1-\lambda)}{1+3 \lambda^{2}} .
$$

The radiative corrections are determined by the functions $g_{n}\left(E_{e}\right)$ and $f_{n}\left(E_{e}\right)$, which are given in Eq. (D-58) of Appendix D.

The functions $(\alpha / \pi) g_{n}\left(E_{e}\right)$ and $(\alpha / \pi) f_{n}\left(E_{e}\right)$ describe the radiative corrections to the lifetime of the neutron and the correlation coefficients $a\left(E_{e}\right)$ and $A\left(E_{e}\right)$, respectively. They are equal to the radiative corrections, calculated in [18]-32]. We show in Appendix D that the correlation coefficient $B\left(E_{e}\right)$ has no radiative corrections to order $\alpha / \pi$. This agrees also well with the results, obtained in [32]. The densities of the radiative corrections $(\alpha / \pi) g_{n}\left(E_{e}\right) \rho_{\beta_{c}^{-}}\left(E_{e}\right)$ and $(\alpha / \pi) f_{n}\left(E_{e}\right) \rho_{\beta_{c}^{-}}\left(E_{e}\right)$, where $\rho_{\beta_{c}^{-}}\left(E_{e}\right)$ is the electron-energy spectrum density Eq.(D-59), are plotted in Fig. 1.

The coefficients $K_{n}\left(E_{e}\right)$ and $Q_{n}\left(E_{e}\right)$ have been introduced in 31, 32] and calculated within the effective quantum field theory, based on the heavy-baryon Chiral Perturbation Theory (HBChPT). Our results of the calculation of the correlation coefficients, carried out to next-to-leading order in the large $M$ expansion (see Appendix A), agree fully with the expressions, calculated in [32].

The correlation coefficient $D\left(E_{e}\right)$ relates to a violation of time reversal invariance. In the SM a non-vanishing correlation coefficient $D\left(E_{e}\right)$ may appear due to long-range [54]-[57] (see also [33]) and short-range [58] mechanisms of time reversal violation. In the long-range mechanism of time reversal violation the correlation coefficient $D\left(E_{e}\right)$ is induced by the electron-proton interaction in the final-state of the decay due to the distortion of the electron wave function in the Coulomb field of the proton [59, 60], the "weak magnetism" and the proton recoil. In the short-range 
mechanism of time reversal violation the correlation coefficient $D\left(E_{e}\right)$ takes a contribution from the CP-violating phase $\delta$ of the CKM quark mixing matrix [9]. According to [58], the contribution of the long-range mechanism of time reversal violation dominates by many orders of magnitude in comparison with the contribution of the short-range one. As has been shown in [54]-57] the correlation coefficient $D\left(E_{e}\right)$ is a function of the electron energy $E_{e}$. Using the results obtained in [54], for the electron kinetic energies $250 \mathrm{keV} \leq T_{e} \leq 455 \mathrm{keV}$ [1, 37, 39] and the axial coupling constant $\lambda=-1.2750$ we obtain that $D\left(E_{e}\right) \sim 10^{-5}$. Hence the contribution of the long-range mechanism of time reversal violation to the correlation coefficient $D\left(E_{e}\right)$ is smaller compared with contributions of order $10^{-4}$, which may be induced by interactions beyond the SM. Recently the correlation coefficient $D\left(E_{e}\right)$ has been calculated within heavy-baryon effective field theory by Ando et al. [61]. The authors have reproduced the result, obtained by Callan and Treiman [54], and have found a correction, which is smaller compared with $10^{-7}$ in the experimental region of electron kinetic energies $250 \mathrm{keV} \leq T_{e} \leq 455 \mathrm{keV}[1,37,39]$.

From Eq.(7) the correlation coefficients under consideration, taking into account the contributions of order $1 / M$ and $\alpha / \pi$, we define as follows

$$
\begin{aligned}
a\left(E_{e}\right) & =a_{0}\left(1+\frac{\alpha}{\pi} f_{n}\left(E_{e}\right)\right)+\frac{1}{M}\left[\frac{2 \lambda(\lambda-(\kappa+1))}{1+3 \lambda^{2}} E_{0}-\frac{4 \lambda(3 \lambda-(\kappa+1))}{1+3 \lambda^{2}} E_{e}\right]-a_{0} \delta \zeta\left(E_{e}\right), \\
A\left(E_{e}\right) & =A_{0}\left(1+\frac{\alpha}{\pi} f_{n}\left(E_{e}\right)\right)+\frac{1}{M}\left[\frac{\lambda^{2}-\kappa \lambda-(\kappa+1)}{1+3 \lambda^{2}} E_{0}-\frac{5 \lambda^{2}-(3 \kappa-4) \lambda-(\kappa+1)}{1+3 \lambda^{2}} E_{e}\right]-A_{0} \delta \zeta\left(E_{e}\right), \\
B\left(E_{e}\right) & =B_{0}+\frac{1}{M}\left[-\frac{2 \lambda(\lambda-(\kappa+1))}{1+3 \lambda^{2}} E_{0}+\frac{7 \lambda^{2}-(3 \kappa+8) \lambda+(\kappa+1)}{1+3 \lambda^{2}} E_{e}-\frac{\lambda^{2}-(\kappa+2) \lambda+(\kappa+1)}{1+3 \lambda^{2}} \frac{m_{e}^{2}}{E_{e}}\right] \\
& -B_{0} \delta \zeta\left(E_{e}\right), \\
K_{n}\left(E_{e}\right) & =\frac{1}{M} \frac{5 \lambda^{2}-(\kappa-4) \lambda-(\kappa+1)}{1+3 \lambda^{2}} E_{e}, \\
Q_{n}\left(E_{e}\right) & =\frac{1}{M}\left[\frac{\lambda^{2}-(\kappa+2) \lambda+(\kappa+1)}{1+3 \lambda^{2}} E_{0}-\frac{7 \lambda^{2}-(\kappa+8) \lambda+(\kappa+1)}{1+3 \lambda^{2}} E_{e}\right] .
\end{aligned}
$$

Using the following expansion

$$
\begin{aligned}
\frac{1}{\zeta\left(E_{e}\right)} & =\left(1-\frac{\alpha}{\pi} g_{n}\left(E_{e}\right)\right)-\delta \zeta\left(E_{e}\right) \\
\delta \zeta\left(E_{e}\right) & =\frac{1}{M} \frac{1}{1+3 \lambda^{2}}\left(\zeta_{1} E_{0}+\zeta_{2} E_{e}+\zeta_{3} \frac{m_{e}^{2}}{E_{e}}\right) \\
\zeta_{1} & =-2 \lambda(\lambda-(\kappa+1)) \\
\zeta_{2} & =10 \lambda^{2}-4(\kappa+1) \lambda+2 \\
\zeta_{3} & =-2 \lambda(\lambda-(\kappa+1))
\end{aligned}
$$

we transcribe the correlation coefficients $a\left(E_{e}\right), A\left(E_{e}\right)$ and $B\left(E_{e}\right)$ into the form, which is similar to that proposed by Wilkinson for the correlation coefficient $A^{(W)}\left(E_{e}\right)$ (see Eq. (20)). We get

$$
\begin{aligned}
& a\left(E_{e}\right)=a_{0}\left\{1+\frac{1}{M} \frac{1}{\left(1-\lambda^{2}\right)\left(1+3 \lambda^{2}\right)}\left(a_{1} E_{0}+a_{2} E_{e}+a_{3} \frac{m_{e}^{2}}{E_{e}}\right)\right\}\left(1+\frac{\alpha}{\pi} f_{n}\left(E_{e}\right)\right), \\
& a_{1}=4 \lambda\left(\lambda^{2}+1\right)(\lambda-(\kappa+1)), \\
& a_{2}=-26 \lambda^{4}+8(\kappa+1) \lambda^{3}-20 \lambda^{2}+8(\kappa+1) \lambda-2, \\
& a_{3}=-2 \lambda\left(\lambda^{2}-1\right)(\lambda-(\kappa+1))
\end{aligned}
$$

and

$$
\begin{aligned}
& A\left(E_{e}\right)=A_{0}\left\{1-\frac{1}{M} \frac{1}{2 \lambda(1+\lambda)\left(1+3 \lambda^{2}\right)}\left(A_{1} E_{0}+A_{2} E_{e}+A_{3} \frac{m_{e}^{2}}{E_{e}}\right)\right\}\left(1+\frac{\alpha}{\pi} f_{n}\left(E_{e}\right)\right), \\
& A_{1}=-\lambda^{4}+\kappa \lambda^{3}+(\kappa+2) \lambda^{2}-\kappa \lambda-(\kappa+1), \\
& A_{2}=5 \lambda^{4}+\kappa \lambda^{3}-(5 \kappa+6) \lambda^{2}+3 \kappa \lambda+(\kappa+1), \\
& A_{3}=-4 \lambda^{2}(\lambda+1)(\lambda-(\kappa+1))
\end{aligned}
$$


and

$$
\begin{aligned}
& B\left(E_{e}\right)=B_{0}\left\{1-\frac{1}{M} \frac{1}{2 \lambda(1-\lambda)\left(1+3 \lambda^{2}\right)}\left(B_{1} E_{0}+B_{2} E_{e}+B_{3} \frac{m_{e}^{2}}{E_{e}}\right)\right\}, \\
& B_{1}=-2 \lambda(\lambda+1)^{2}(\lambda-(\kappa+1)), \\
& B_{2}=\lambda^{4}-(\kappa-4) \lambda^{3}-(5 \kappa+2) \lambda^{2}-(3 \kappa+4) \lambda+(\kappa+1), \\
& B_{3}=\left(\lambda^{2}-1\right)(\lambda-1)(\lambda-(\kappa+1)) .
\end{aligned}
$$

For the derivation of Eq.(11), Eq.(12) and Eq.(13) we have neglected the terms of order $(\alpha / \pi)\left(E_{0} / M\right) \sim 10^{-6}$, which are smaller compared with contributions of order $10^{-4}$ beyond the SM.

In order to estimate the values of the obtained $1 / M$ corrections we calculate them at $\lambda=-1.2750$. This gives

$$
\begin{aligned}
\delta \zeta\left(E_{e}\right) & =\frac{1}{M} \frac{1}{1+3 \lambda^{2}}\left(\zeta_{1} E_{0}+\zeta_{2} E_{e}+\zeta_{3} \frac{m_{e}^{2}}{E_{e}}\right)=-3.57 \times 10^{-3}+9.90 \times 10^{-3} \frac{E_{e}}{E_{0}}-1.41 \times 10^{-3} \frac{m_{e}}{E_{e}}, \\
\frac{\delta a\left(E_{e}\right)}{a_{0}} & =\frac{1}{M} \frac{1}{\left(1-\lambda^{2}\right)\left(1+3 \lambda^{2}\right)}\left(a_{1} E_{0}+a_{2} E_{e}+a_{3} \frac{m_{e}^{2}}{E_{e}}\right)=-3.00 \times 10^{-2}+8.59 \times 10^{-2} \frac{E_{e}}{E_{0}}+1.41 \times 10^{-3} \frac{m_{e}}{E_{e}}, \\
\frac{\delta A\left(E_{e}\right)}{A_{0}} & =-\frac{1}{M} \frac{1}{2 \lambda(1+\lambda)\left(1+3 \lambda^{2}\right)}\left(A_{1} E_{0}+A_{2} E_{e}+A_{3} \frac{m_{e}^{2}}{E_{e}}\right)=3.44 \times 10^{-4}+1.46 \times 10^{-2} \frac{E_{e}}{E_{0}}+1.41 \times 10^{-3} \frac{m_{e}}{E_{e}}, \\
\frac{\delta B\left(E_{e}\right)}{B_{0}} & =-\frac{1}{M} \frac{1}{2 \lambda(1-\lambda)\left(1+3 \lambda^{2}\right)}\left(B_{1} E_{0}+B_{2} E_{e}+B_{3} \frac{m_{e}^{2}}{E_{e}}\right)=-4.66 \times 10^{-5}-2.97 \times 10^{-4} \frac{E_{e}}{E_{0}}+1.36 \times 10^{-4} \frac{m_{e}}{E_{e}}, \\
K_{n}\left(E_{e}\right) & =7.14 \times 10^{-4} \frac{E_{e}}{E_{0}}, \\
Q_{n}\left(E_{e}\right) & =3.19 \times 10^{-3}-7.27 \times 10^{-3} \frac{E_{e}}{E_{0}} .
\end{aligned}
$$

Due to strong dependence on the axial coupling constant $\lambda$ the numerical values of the contributions of the "weak magnetism" and the proton recoil, calculated at $\lambda=-1.2750$, vary from $10^{-5}$ to $10^{-1}$ for energy independent and energy dependent terms.

Summary. The correlation coefficients of the electron-energy and angular distribution of the neutron $\beta^{--}$-decay with a polarized neutron and unpolarised decay proton and electron are calculated by taking into account a complete set of the $1 / M$ corrections, caused by the "weak magnetism" and the proton recoil, and the radiative corrections of order $\alpha / \pi$, calculated to leading order in the large $M$ expansion. The obtained expressions for the correlation coefficients should be used as a theoretical background for contributions of order $10^{-4}$ of interactions beyond the SM, calculated in Appendix G (see Eq. G-11 and Eq. G-12). Contributions of order $10^{-4}$ of interactions beyond the SM may be determined by measuring the asymmetries $A_{\exp }\left(E_{e}\right), B_{\exp }\left(E_{e}\right)$ and $C_{\exp }$ between the neutron spin and the 3 -momenta of the decay particles, the electron-proton energy distribution $a\left(E_{e}, T_{p}\right)$ and the proton-energy spectrum $a\left(T_{p}\right)$, related to correlations between the $3-$ momenta of the electron and proton, and the lifetime of the neutron $\tau_{n}$ (see section [X]).

\section{STANDARD MODEL ANALYSIS OF EXPERIMENTAL DETERMINATION OF CORRELATION COEFFICIENT $A_{0}$. ELECTRON ASYMMETRY $A_{\exp }\left(E_{e}\right)$}

For the experimental determination of the correlation coefficient $A_{0}$, defining correlations between the neutron spin and the electron 3-momentum in the SM to leading order in the large $M$ expansion [1], the directions of the emission of the antineutrino are not fixed and one has to integrate over the antineutrino $3-$ momentum $\vec{k}$. As a result we arrive at the following electron-energy and angular distribution [1, 37]

$$
\begin{aligned}
\frac{d^{2} \lambda_{n}\left(E_{e}, \vec{k}_{e}, \vec{\xi}_{n}\right)}{d E_{e} d \Omega_{e}}= & \left(1+3 \lambda^{2}\right) \frac{G_{F}^{2}\left|V_{u d}\right|^{2}}{8 \pi^{4}}\left(E_{0}-E_{e}\right)^{2} \sqrt{E_{e}^{2}-m_{e}^{2}} E_{e} F\left(E_{e}, Z=1\right) \zeta\left(E_{e}\right) \\
& \times\left(1+A^{(W)}\left(E_{e}\right)\left(1+\frac{\alpha}{\pi} f_{n}\left(E_{e}\right)\right) \vec{\xi}_{n} \cdot \vec{\beta}\right)
\end{aligned}
$$

where we have denoted

$$
A\left(E_{e}\right)+\frac{1}{3} Q_{n}\left(E_{e}\right)=A^{(W)}\left(E_{e}\right)\left(1+\frac{\alpha}{\pi} f_{n}\left(E_{e}\right)\right),
$$


$d \Omega_{e}=2 \pi \sin \theta_{e} d \theta_{e}$ is an infinitesimal solid angle of the electron 3 -momentum with respect to the neutron spin and $\vec{\xi}_{n} \cdot \vec{\beta}=P \beta \cos \theta_{e}$ with the neutron polarization $P=\left|\vec{\xi}_{n}\right| \leq 1$. The correlation coefficients $A\left(E_{e}\right)$ and $Q_{n}\left(E_{e}\right)$ are given in Eq.(12) and Eq.(9), respectively. The contribution, proportional to $Q_{n}\left(E_{e}\right)$, with structure $\vec{\xi}_{n} \cdot \vec{k}_{e} / E_{e}$ we obtain having integrated the term with the structure $\left(\vec{\xi}_{n} \cdot \vec{k}\right)\left(\overrightarrow{k_{e}} \cdot \vec{k}\right) / E_{e} E^{2}$ in Eq. (6) over directions of the antineutrino 3-momentum $\vec{k}$.

The asymmetry, which may be used for the experimental determination of the axial coupling constant $\lambda$ and the correlation coefficient $A_{0}$, takes the form

$$
A_{\exp }\left(E_{e}\right)=\frac{N^{+}\left(E_{e}\right)-N^{-}\left(E_{e}\right)}{N^{+}\left(E_{e}\right)+N^{-}\left(E_{e}\right)}=\frac{1}{2} A^{(W)}\left(E_{e}\right)\left(1+\frac{\alpha}{\pi} f_{n}\left(E_{e}\right)\right) P \beta\left(\cos \theta_{1}+\cos \theta_{2}\right),
$$

where $N^{ \pm}\left(E_{e}\right)$ are the numbers of events of the emission of the electron forward $(+)$ and backward $(-)$ with respect to the neutron spin into the solid angle $\Delta \Omega_{12}=2 \pi\left(\cos \theta_{1}-\cos \theta_{2}\right)$ with $0 \leq \varphi \leq 2 \pi$ and $\theta_{1} \leq \theta_{e} \leq \theta_{2}$. They are determined by 62 ]

$$
\begin{aligned}
& N^{+}\left(E_{e}\right)=2 \pi N\left(E_{e}\right) \int_{\theta_{1}}^{\theta_{2}}\left(1+A^{(W)}\left(E_{e}\right)\left(1+\frac{\alpha}{\pi} f_{n}\left(E_{e}\right)\right) P \beta \cos \theta_{e}\right) \sin \theta_{e} d \theta_{e}= \\
& =2 \pi N\left(E_{e}\right)\left(1+\frac{1}{2} A^{(W)}\left(E_{e}\right)\left(1+\frac{\alpha}{\pi} f_{n}\left(E_{e}\right)\right) P \beta\left(\cos \theta_{1}+\cos \theta_{2}\right)\right)\left(\cos \theta_{1}-\cos \theta_{2}\right), \\
& N^{-}\left(E_{e}\right)=2 \pi N\left(E_{e}\right) \int_{\pi-\theta_{1}}^{\pi-\theta_{2}}\left(1+A^{(W)}\left(E_{e}\right)\left(1+\frac{\alpha}{\pi} f_{n}\left(E_{e}\right)\right) P \beta \cos \theta_{e}\right) \sin \theta_{e} d \theta_{e}= \\
& =2 \pi N\left(E_{e}\right)\left(1-\frac{1}{2} A^{(W)}\left(E_{e}\right)\left(1+\frac{\alpha}{\pi} f_{n}\left(E_{e}\right)\right) P \beta\left(\cos \theta_{1}+\cos \theta_{2}\right)\right)\left(\cos \theta_{1}-\cos \theta_{2}\right),
\end{aligned}
$$

where $N\left(E_{e}\right)$ is the normalization factor equal to

$$
N\left(E_{e}\right)=\left(1+3 \lambda^{2}\right) \frac{G_{F}^{2}\left|V_{u d}\right|^{2}}{8 \pi^{4}}\left(E_{0}-E_{e}\right)^{2} \sqrt{E_{e}^{2}-m_{e}^{2}} E_{e} F\left(E_{e}, Z=1\right) \zeta\left(E_{e}\right)
$$

The correlation coefficient $A^{(W)}\left(E_{e}\right)$ is

$$
\begin{aligned}
& A^{(W)}\left(E_{e}\right)=A_{0}\left\{1-\frac{1}{M} \frac{1}{2 \lambda(1+\lambda)\left(1+3 \lambda^{2}\right)}\left(A_{1}^{(W)} E_{0}+A_{2}^{(W)} E_{e}+A_{3}^{(W)} \frac{m_{e}^{2}}{E_{e}}\right)\right\} \\
& A_{1}^{(W)}=\frac{2}{3}\left(-3 \lambda^{3}+(3 \kappa+5) \lambda^{2}-(2 \kappa+1) \lambda-(\kappa+1)\right)=-2(\lambda-(\kappa+1))\left(\lambda^{2}-\frac{2}{3} \lambda-\frac{1}{3}\right), \\
& A_{2}^{(W)}=\frac{2}{3}\left(-3 \lambda^{4}+(3 \kappa+12) \lambda^{3}-(9 \kappa+14) \lambda^{2}+(5 \kappa+4) \lambda+(\kappa+1)\right)=-2(\lambda-(\kappa+1))\left(\lambda^{3}-3 \lambda^{2}+\frac{5}{3} \lambda+\frac{1}{3}\right), \\
& A_{3}^{(W)}=-4 \lambda^{2}(\lambda+1)(\lambda-(\kappa+1)) .
\end{aligned}
$$

It agrees well with the result, obtained by Wilkinson [34, 63]. We note that the correlation coefficient $A\left(E_{e}\right)+\frac{1}{3} Q_{n}\left(E_{e}\right)$ differs from the Wilkinson correlation coefficient $A^{(W)}\left(E_{e}\right)$ by the contribution of the radiative corrections, described by the function $(\alpha / \pi) f_{n}\left(E_{e}\right)$. In the replacement $A\left(E_{e}\right)+\frac{1}{3} Q_{n}\left(E_{e}\right) \rightarrow A^{(W)}\left(E_{e}\right)\left(1+(\alpha / \pi) f_{n}\left(E_{e}\right)\right)$ we have neglected the contributions of order $(\alpha / \pi)\left(E_{0} / M\right) \sim 10^{-6}$, which are smaller compared with contributions of order $10^{-4}$ of our interest. The contribution to the correlation coefficient $A^{(W)}\left(E_{e}\right)$ of the "weak magnetism" and the proton recoil, calculated at $\lambda=-1.2750$, is equal to

$$
\begin{aligned}
\frac{\delta A^{(W)}\left(E_{e}\right)}{A_{0}} & =-\frac{1}{M} \frac{1}{2 \lambda(1+\lambda)\left(1+3 \lambda^{2}\right)}\left(A_{1}^{(W)} E_{0}+A_{2}^{(W)} E_{e}+A_{3}^{(W)} \frac{m_{e}^{2}}{E_{e}}\right)= \\
& =-8.56 \times 10^{-3}+3.49 \times 10^{-2} \frac{E_{e}}{E_{0}}+1.41 \times 10^{-3} \frac{m_{e}}{E_{e}}
\end{aligned}
$$

In the experimentally used region of electron kinetic energies $250 \mathrm{keV} \leq T_{e} \leq 455 \mathrm{keV}$ [1, 37, 39] the radiative corrections $(\alpha / \pi) f_{n}\left(E_{e}\right)$ vary over the region $1.53 \times 10^{-3} \geq(\alpha / \pi) f_{n}\left(E_{e}\right) \geq 1.04 \times 10^{-3}$ and increase the absolute value of the correlation coefficient $A^{(W)}\left(E_{e}\right)$.

Summary. The Wilkinson expression for the correlation coefficient $A^{(\mathrm{W})}\left(E_{e}\right)$ (see Eq. (20)), taking into account a complete set of the $1 / M$ corrections from the "weak magnetism" and the proton recoil, is improved by the account for 
the radiative corrections, described by the function $2.81 \times 10^{-3} \geq(\alpha / \pi) f_{n}\left(E_{e}\right) \geq 0.62 \times 10^{-3}$ for the electron energies $m_{e} \leq E_{e} \leq E_{0}$ (see Eq.(D-58)). The contribution of the radiative corrections increases the absolute value of the correlation coefficient $A^{(\mathrm{W})}\left(E_{e}\right)$. The results, obtained in this section, should be used as a theoretical background for the experimental determination of contributions of order $10^{-4}$ of interactions beyond the SM from the experimental data on the electron asymmetry $A_{\exp }\left(E_{e}\right)$ (see section [X).

\section{STANDARD MODEL ANALYSIS OF EXPERIMENTAL DETERMINATION OF CORRELATION COEFFICIENT $B_{0}$. ANTINEUTRINO ASYMMETRY $B_{\exp }\left(E_{e}\right)$}

In the SM to leading order in the large $M$ expansion the correlations between the neutron spin and the antineutrino 3-momentum are defined by the correlation coefficient $B_{0}$ [1], which may be determined from the experimental data on the asymmetry $B_{\exp }\left(E_{e}\right)$, defined by 38 ]

$$
B_{\exp }\left(E_{e}\right)=\frac{N^{--}\left(E_{e}\right)-N^{++}\left(E_{e}\right)}{N^{--}\left(E_{e}\right)+N^{++}\left(E_{e}\right)} .
$$

It defines the asymmetry of the emission of the antineutrinos into the forward and backward hemisphere with respect to the neutron spin, where $N^{\mp \mp}\left(E_{e}\right)$ is the number of events of the emission of the electron-proton pairs as functions of the electron energy $E_{e}$. The signs $(++)$ and $(--)$ show that the electron-proton pairs were emitted parallel $(++)$ and antiparallel $(--)$ to a direction of the neutron spin. This means that antineutrinos were emitted antiparallel $(++)$ and parallel $(--)$ to a direction of the neutron spin. The number of events $N^{--}\left(E_{e}\right)$ and $N^{++}\left(E_{e}\right)$ are defined by the electron-energy and angular distribution of the neutron $\beta^{-}$-decay, integrated over the forward and backward hemisphere relative to the neutron spin, respectively.

The integration region for the electron-proton pairs, emitted parallel to a direction $(++)$ of the neutron spin, is defined by the constraints [64]: $\vec{\xi}_{n} \cdot \vec{k}_{e}=P k_{e} \cos \theta_{e}>0$ and $\vec{\xi}_{n} \cdot \vec{k}_{p}=\vec{\xi}_{n} \cdot\left(-\vec{k}_{e}-\vec{k}\right)=P E\left(-r \cos \theta_{e}-\cos \theta\right)>0$ or $-r \cos \theta_{e}>\cos \theta$, where $r=k_{e} / E=\sqrt{E_{e}^{2}-m_{e}^{2}} /\left(E_{0}-E_{e}\right)$ and $P=\left|\vec{\xi}_{n}\right| \leq 1$ is the neutron polarization. For $N^{++}\left(E_{e}\right)$ and $r<1$ we obtain the following expression

$$
\begin{aligned}
& N^{++}\left(E_{e}\right)=2 \pi N\left(E_{e}\right)\left\{\left(1-\frac{1}{4} a \beta+\frac{1}{2} P \beta\left(A-\frac{1}{3} K_{n} \beta\right)-\frac{1}{2} P\left(B-\frac{1}{3} Q_{n} \beta\right)\right)\right. \\
& \left.-\frac{1}{2} r\left(1+\frac{2}{3} P A \beta\right)+\frac{1}{8} r^{2}\left(a \beta+\frac{4}{3} P B+\frac{4}{5} P K_{n} \beta^{2}\right)-\frac{1}{15} r^{3} P Q_{n} \beta-\frac{1}{8} a_{0} \beta^{2} r\left(1-r^{2}\right) \frac{E_{e}}{M}\right\}
\end{aligned}
$$

where $N\left(E_{e}\right)$ is the normalization factor Eq.(19). For $r>1$ the upper limit of the integration over $\cos \theta_{e}$ is restricted by $\cos \theta_{e} \leq 1 / r$. The result of the interaction is

$N^{++}\left(E_{e}\right)=2 \pi N\left(E_{e}\right)\left\{\frac{1}{2} \frac{1}{r}\left(1-\frac{2}{3} P B\right)-\frac{1}{8} \frac{1}{r^{2}}\left(a \beta-\frac{4}{3} P A \beta-\frac{4}{5} P Q_{n} \beta\right)-\frac{1}{15} \frac{1}{r^{3}} P K_{n} \beta^{2}+\frac{1}{8} a_{0} \beta^{2} \frac{1}{r}\left(1-\frac{1}{r^{2}}\right) \frac{E_{e}}{M}\right\}$.

For the calculation of $N^{--}\left(E_{e}\right)$ we have to integrate over the region [64] : $\vec{\xi}_{n} \cdot \vec{k}_{e}=P k_{e} \cos \theta_{e}<0$ and $\vec{\xi}_{n} \cdot \vec{k}_{p}=$ $P E\left(-r \cos \theta_{e}-\cos \theta\right)<0$ or $\cos \theta>-r \cos \theta_{e}$. The number of events $N^{--}\left(E_{e}\right)$ for $r<1$ is given by

$$
\begin{aligned}
& N^{--}\left(E_{e}\right)=2 \pi N\left(E_{e}\right)\left\{\left(1-\frac{1}{4} a \beta-\frac{1}{2} P \beta\left(A-\frac{1}{3} K_{n} \beta\right)+\frac{1}{2} P\left(B-\frac{1}{3} Q_{n} \beta\right)\right)\right. \\
& \left.-\frac{1}{2} r\left(1-\frac{2}{3} P A \beta\right)+\frac{1}{8} r^{2}\left(a \beta-\frac{4}{3} P B-\frac{4}{5} P K_{n} \beta^{2}\right)+\frac{1}{15} r^{3} P Q_{n} \beta-\frac{1}{8} a_{0} \beta^{2} r\left(1-r^{2}\right) \frac{E_{e}}{M}\right\} .
\end{aligned}
$$

For $r>1$ the lower limit of the integration over $\cos \theta_{e}$ is restricted by $\cos \theta_{e}>-1 / r$. The number of events $N^{--}\left(E_{e}\right)$, calculated for $r>1$, is equal to

$N^{--}\left(E_{e}\right)=2 \pi N\left(E_{e}\right)\left\{\frac{1}{2} \frac{1}{r}\left(1+\frac{2}{3} P B\right)-\frac{1}{8} \frac{1}{r^{2}}\left(a \beta+\frac{4}{3} P A \beta+\frac{4}{5} P Q_{n} \beta\right)+\frac{1}{15} \frac{1}{r^{3}} P K_{n} \beta^{2}+\frac{1}{8} a_{0} \beta^{2} \frac{1}{r}\left(1-\frac{1}{r^{2}}\right) \frac{E_{e}}{M}\right\}$.

Using our formulas for the numbers of events we calculate the asymmetry $B_{\exp }\left(E_{e}\right)$. For $r \leq 1$ or $0 \leq T_{e} \leq$ $\left(E_{0}-m_{e}\right)^{2} / 2 E_{0}=236 \mathrm{keV}$ and for $r \geq 1$ or $\left(E_{0}-m_{e}\right)^{2} / 2 E_{0}=236 \mathrm{keV} \leq T_{e} \leq E_{0}-m_{e}$ the asymmetry $B_{\exp }\left(E_{e}\right)$ is 


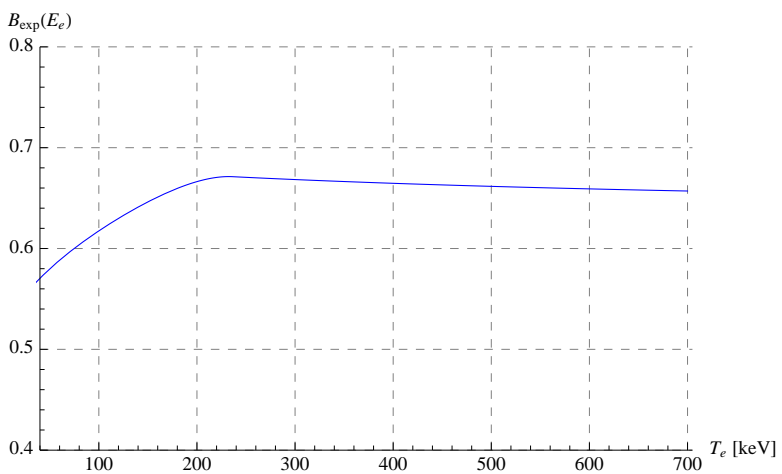

FIG. 2: The antineutrino asymmetry $B_{\exp }\left(E_{e}\right)$, including a complete set of the $1 / M$ corrections, caused by the "weak magnetism" and the proton recoil, and radiative corrections of order $\alpha / \pi$, calculated to leading order in the large $M$ expansion; $T_{e}=E_{e}-m_{e}$ is the electron kinetic energy.

equal to

$$
B_{\exp }^{(r<1)}\left(E_{e}\right)=\frac{2 P}{3} \frac{\left(3-r^{2}\right) B-(3-2 r) A \beta+\left(1-\frac{3}{5} r^{2}\right) K_{n} \beta^{2}-\left(1-\frac{2}{5} r^{3}\right) Q_{n} \beta}{(4-2 r)-\left(1-\frac{1}{2} r^{2}\right) a \beta-\frac{1}{2} a_{0} \beta^{2} r\left(1-r^{2}\right) \frac{E_{e}}{M}}
$$

and

$$
B_{\exp }^{(r>1)}\left(E_{e}\right)=\frac{2 P}{3} \frac{B-\frac{1}{2}\left(A+\frac{3}{5} Q_{n}\right) \frac{\beta}{r}+\frac{1}{5} K_{n} \frac{\beta^{2}}{r^{2}}}{1-a \frac{\beta}{4 r}+\frac{1}{4} a_{0} \beta^{2}\left(1-\frac{1}{r^{2}}\right) \frac{E_{e}}{M}},
$$

respectively. At $r=1$ or $T_{e}=\left(E_{0}-m_{e}\right)^{2} / 2 E_{0}=236 \mathrm{keV}$ the asymmetry $B_{\exp }\left(E_{e}\right)$ is continuous. To leading order in the large $M$ expansion the asymmetry $B_{\exp }\left(E_{e}\right)$ reduces to the form

$$
\left.B_{\exp }\left(E_{e}\right)\right|_{M \rightarrow \infty}=\frac{2 P}{3}\left\{\begin{array}{c}
\frac{B\left(\frac{3}{2}-\frac{1}{2} r^{2}\right)-\left(\frac{3}{2}-r\right) A \beta}{(2-r)-\frac{1}{2}\left(1-\frac{1}{2} r^{2}\right) a \beta}, r \leq 1 \\
\frac{B-\frac{1}{2} A \frac{\beta}{r}}{1-\frac{1}{4} a \frac{\beta}{r}}, r \geq 1,
\end{array}\right.
$$

where the correlation coefficients $B, A$ and $a$ are equal to

$$
B=B_{0} \quad, \quad A=A_{0}\left(1+\frac{\alpha}{\pi} f_{n}\left(E_{e}\right)\right) \quad, \quad a=a_{0}\left(1+\frac{\alpha}{\pi} f_{n}\left(E_{e}\right)\right) .
$$

In Fig. 2 we plot the asymmetry $B_{\exp }\left(E_{e}\right)$, given by Eq.(27) and Eq.(28) and obtained in the SM with the account for the contributions of the $1 / M$ corrections, caused by the "weak magnetism" and the proton recoil and the radiative corrections of order $\alpha / \pi$, calculated to leading order in the large $M$ expansion and described by the function $(\alpha / \pi) f_{n}\left(E_{e}\right)$. In [38, 39] for the experimental determination of the correlation coefficient $B_{0}$ the experimental data on the asymmetry $B_{\exp }\left(E_{e}\right)$ were fitted by Eq.(29) with the replacement $B \rightarrow B_{0}, A \rightarrow A_{0}$ and $a \rightarrow a_{0}$, respectively. Such an asymmetry was calculated in [64]. The "weak magnetism", the proton recoil and the radiative corrections to Eq. (29) with the replacement $B \rightarrow B_{0}, A \rightarrow A_{0}$ and $a \rightarrow a_{0}$ have been calculated numerically in 65. They are positive and negative for $T_{e}<470 \mathrm{keV}$ and $T_{e}>470 \mathrm{keV}$, respectively. The corrections, calculated in this paper, are positive and of order of magnitude larger compared with the absolute values of the corrections, calculated in [65].

The theoretical value $B_{0}=0.9871(1)$ of the correlation coefficient $B_{0}$, calculated for $\lambda=-1.2750(9)$, agrees within 1.5 standard deviations with the experimental values $B_{0}^{(\exp )}=0.9802(50), B_{0}^{(\exp )}=0.9821(40)$ and $B_{0}^{(\exp )}=$ 0.9894(83), obtained in [38, 39] (see also [1]), 66] and [67], respectively, and within two standard deviations with the experimental one $B_{0}^{(\exp )}=0.967(12)$, obtained in [68]. 
Summary. The antineutrino asymmetry $B_{\exp }\left(E_{e}\right)$, calculated in [64] and used in [38, 39] for the experimental determination of the correlation coefficient $B_{0}^{(\exp )}=0.9802(50)$, is improved by the contributions of a complete set of the $1 / M$ corrections, caused by the "weak magnetism" and the proton recoil, and the radiative corrections $(\alpha / \pi) f_{n}\left(E_{e}\right)$, calculated to leading order in the large $M$ expansion. The obtained expression of the antineutrino asymmetry $B_{\exp }\left(E_{e}\right)$ should be used as a theoretical background for the experimental determination of contributions of order $10^{-4}$ of interactions beyond the SM (see section IX).

\section{STANDARD MODEL ANALYSIS OF EXPERIMENTAL DETERMINATION OF CORRELATION COEFFICIENT $a_{0}$. ELECTRON-PROTON ENERGY DISTRIBUTION $a\left(E_{e}, T_{p}\right)$ AND PROTON-ENERGY SPECTRUM $a\left(T_{p}\right)$}

For the experimental determination of the correlation coefficient $a_{0}$ the correlation coefficient $a\left(E_{e}\right)$, given by Eq. (11), can be hardly used, since the antineutrino is hard to detect. Thus, for the determination of $a_{0}$ and the contributions of interactions beyond the SM one should measure correlations of the 3-momenta of the decay charged particles. Using the results, obtained in Appendix I, the electron-proton energy spectrum for the neutron $\beta^{-}-$decay with unpolarised particles can be given in the following form

$$
\frac{d^{2} \mathrm{BR}_{\beta_{c}^{-}}\left(E_{e}, T_{p}\right)}{d E_{e} d T_{p}}=\left(\tau_{n}\right)_{\mathrm{SM}} M\left(1+3 \lambda^{2}\right) \frac{G_{F}^{2}\left|V_{u d}\right|^{2}}{4 \pi^{3}} a\left(E_{e}, T_{p}\right)\left(1+\frac{\alpha}{\pi} g_{n}\left(E_{e}\right)\right) F\left(E_{e}, Z=1\right) E_{e}
$$

where $\left(\tau_{n}\right)_{\mathrm{SM}}$ is the theoretical lifetime of the neutron, calculated in the SM (see section VIII) and $T_{p}$ is the kinetic energy of the proton varying from zero to its maximal value $\left(T_{p}\right)_{\max }=\left(m_{n}-m_{p}\right)^{2}-m_{e}^{2} / 2 m_{n}=\left(E_{0}^{2}-m_{e}^{2}\right) / 2 M=$ $0.751 \mathrm{keV}$, i.e. $0 \leq T_{p} \leq 0.751 \mathrm{keV}$. The limits of the integration over the electron energy $E_{e}$, i.e. $\left(E_{e}\right)_{\min } \leq E_{e} \leq$ $\left(E_{e}\right)_{\max }$, are the functions of the proton kinetic energy $T_{p}$. They are adduced in Appendix I. The electron-proton energy distribution $a\left(E_{e}, T_{p}\right)$ is defined by (see Appendix I)

$$
a\left(E_{e}, T_{p}\right)=\zeta_{1}\left(E_{e}, T_{p}\right)+a_{0}\left(1+\frac{1}{1-\lambda^{2}} \frac{E_{0}}{M}\right)\left(1+\frac{\alpha}{\pi} f_{n}\left(E_{e}\right)\right) \zeta_{2}\left(E_{e}, T_{p}\right) .
$$

The functions $\zeta_{1}\left(E_{e}, T_{p}\right)$ and $\zeta_{2}\left(E_{e}, T_{p}\right)$ are given by in Appendix I. They are calculated to leading order in the large $M$ and do not contain the radiative corrections. In the electron energy region $\left(E_{e}\right)_{\min } \leq E_{e} \leq\left(E_{e}\right)_{\max }$ the contribution of the radiative corrections $(\alpha / \pi) f_{n}\left(E_{e}\right)$ to the electron-proton energy distribution $a\left(E_{e}, T_{p}\right)$ relative to the correlation coefficient $a_{0}$ is of order $10^{-3}$. The account for the radiative corrections is very important for a correct experimental determination of contributions of order $10^{-4}$ of interactions beyond the SM.

Integrating the electron-proton energy spectrum Eq.31) over the electron energy $\left(E_{e}\right)_{\min } \leq E_{e} \leq\left(E_{e}\right)_{\max }$ we obtain the proton-energy spectrum

$$
\frac{d \mathrm{BR}_{\beta_{c}^{-}}\left(T_{p}\right)}{d T_{p}}=\left(\tau_{n}\right)_{\mathrm{SM}} M\left(1+3 \lambda^{2}\right) \frac{G_{F}^{2}\left|V_{u d}\right|^{2}}{4 \pi^{3}} a\left(T_{p}\right)
$$

where $a\left(T_{p}\right)$ is defined by

$$
a\left(T_{p}\right)=g_{1}\left(T_{p}\right)+a_{0}\left(1+\frac{1}{1-\lambda^{2}} \frac{E_{0}}{M}\right) g_{2}\left(T_{p}\right)
$$

The functions $g_{1}\left(T_{p}\right)$ and $g_{2}\left(T_{p}\right)$ are given in Appendix I. Recently the correlation coefficient $a_{0}$ and the axial coupling constant $\lambda$ have been determined from the proton-energy spectrum by Byrne et al. [40]. The obtained value $a_{0}^{(\exp )}=$ $-0.1054(55)$ can be fitted by the axial coupling constant $\lambda=-1.271(18)$. In turn, the experimental value $a_{0}^{(\exp )}=$ $-0.1017(51)$, obtained in [41], defines the axial coupling constant equal to $\lambda=-1.259(17)$. These experimental values agree with the theoretical value of the correlation coefficient $a_{0}=-0.1065(3)$, calculated at $\lambda=-1.2750(9)$, and with the axial coupling constant $\lambda=-1.2750(9)$ within one standard deviation.

From the point of view of the experimental determination of contributions of order $10^{-4}$ of interactions beyond the SM (see a discussion in section VII) the experimental accuracy of the determination of the axial coupling constant $\lambda$ by measuring the proton-energy spectrum as well as the electron-proton energy distribution $a\left(E_{e}, T_{p}\right)$ should be improved by more than two orders of magnitude in comparison with the accuracy of Byrne's experiment [40]. There are three major, funded experiments, which are currently attempting to do this. They are i) aSPECT experiment at Institute Laue-Langevin (ILL) in Grenoble, invented to perform precise measurements of the correlation coefficient $a_{0}$ by measuring the proton-energy spectrum in the decay of unpolarised neutrons, ii) aCORN at the National Institute 
of Standards and Technology (NIST) and iii) Nab at the new Spallation Neutron Source (SNS) in Oak Ridge at Tennessee [69]. The expected experimental accuracy of the determination of the correlation coefficient $a_{0}$ in the aCORN and Nab experiments is better than $1 \%$ (see also [70]).

We have discussed the experimental determination of the correlation coefficient $a_{0}$ by measuring the electronproton energy distribution $a\left(E_{e}, T_{p}\right)$ and the proton-energy spectrum $a\left(T_{p}\right)$. However, there are another possibilities to determine experimentally the correlation coefficient $a_{0}$. It can be extracted from the experimental data on the $\left(T_{e}, \cos \theta_{e \bar{\nu}}\right)$ and $\left(T_{e}, \cos \theta_{e p}\right)$ distributions [71], where $\theta_{e \bar{\nu}}$ and $\theta_{e p}$ are angles of the electron-antineutrino and electronproton correlations, respectively.

Summary. The electron-proton energy distribution $a\left(E_{e}, T_{p}\right)$ is calculated by taking into account a complete set of the $1 / M$ corrections, caused by the "weak magnetism" and the proton recoil, and the radiative corrections of order $\alpha / \pi$, calculated to leading order in the large $M$ expansion. The proton-energy spectrum is improved in comparison with that, used in [40], by the account for the $1 / M$ and radiative corrections. The obtained expression for the electron-proton energy distribution $a\left(E_{e}, T_{p}\right)$ as well as the proton-energy spectrum $a\left(T_{p}\right)$ should provide a theoretical background for the experimental determination of contributions of order $10^{-4}$ of interactions beyond the SM (see section IX). For the analysis of contributions of interactions beyond the SM we propose to multiply the electron-proton energy spectrum of the rate of the neutron $\beta^{-}$-decay by the lifetime of the neutron $\tau_{n}$, calculated with the account for the contributions of interactions beyond the SM (see Appendix G). As a result the analysis of the contributions of vector and axial-vector interactions beyond the SM by means of the electron-proton energy distribution $\tau_{n} a\left(E_{e}, T_{p}\right)$ and the proton-energy spectrum $\tau_{n} a\left(T_{p}\right)$ reduces to the analysis of these contributions to the axial coupling constant $\lambda$ only (see section $\amalg$ (X).

\section{STANDARD MODEL ANALYSIS OF EXPERIMENTAL DETERMINATION OF CORRELATION COEFFICIENT $C_{0}$. PROTON ASYMMETRY $C_{\exp }$}

The correlations between the neutron spin and the proton 3-momentum are described by the correlation coefficient $C$ [72]-76]. It defines the proton recoil asymmetry $C_{0}=-x_{C}\left(A_{0}+B_{0}\right)$, where $x_{C}$ is the theoretical numerical factor, calculated within the SM to leading order in the large $M$ expansion. The first measurement of the correlation coefficient $C_{0}^{(\exp )}=-0.2377(26)$ has been performed by Schumann et al. [42]. The angular distribution of the probability of the neutron $\beta^{-}$-decay, related to the proton recoil asymmetry, is given by 72$]$

$$
4 \pi \frac{d W\left(\theta_{p}\right)}{d \Omega_{p}}=1+2 P C \cos \theta_{p}
$$

where $d \Omega_{p}=2 \pi \sin \theta_{p} d \theta_{p}$ is the infinitesimal solid angle of the proton 3 -momentum with respect to the neutron polarization $\vec{\xi}_{n}$, i.e. $\vec{\xi}_{n} \cdot \vec{k}_{p}=P k_{p} \cos \theta_{p}$ and $P=\left|\vec{\xi}_{n}\right|$ is the neutron polarization. The correlation coefficient $C$, calculated at the account for the $1 / M$ and $\alpha / \pi$-radiative corrections, is equal to (see Appendix I Eq. (I-30)

$$
\begin{aligned}
& C=-\frac{1}{2} \frac{X_{8}}{X_{1}}\left(A_{0}+B_{0}\right)+\frac{1}{2} \frac{X_{9}}{X_{1}} A_{0}+\frac{\alpha}{\pi} \frac{1}{2} \frac{X_{10}}{X_{1}} A_{0}-\frac{\alpha}{\pi} \frac{1}{2} \frac{X_{11}}{X_{1}} B_{0}+\frac{1}{M} \frac{1}{1+3 \lambda^{2}}\left(\lambda \frac{1}{2} \frac{X_{12}}{X_{1}}-(\kappa+1) \lambda \frac{1}{2} \frac{X_{13}}{X_{1}}\right. \\
& \left.-(2 \kappa+1) \lambda \frac{1}{2} \frac{X_{14}}{X_{1}}-\lambda(1+\lambda) \frac{1}{2} \frac{X_{15}+Y_{3}}{X_{1}}+\lambda(1-\lambda) \frac{1}{2} \frac{X_{16}+Y_{4}}{X_{1}}\right)+\left(A_{0}+B_{0}\right) \frac{X_{8}}{X_{1}}\left\{\frac{\alpha}{\pi} \frac{1}{2} \frac{X_{2}}{X_{1}}+\frac{1}{M} \frac{1}{1+3 \lambda^{2}}\right. \\
& \left.\times\left(\frac{1}{2} \frac{X_{3}}{X_{1}}+\left(1+3 \lambda^{2}\right) \frac{1}{2} \frac{X_{4}+Y_{1}}{X_{1}}-\left(1-\lambda^{2}\right) \frac{1}{2} \frac{X_{5}+Y_{2}}{X_{1}}+\left(\lambda^{2}+2(\kappa+1) \lambda\right) \frac{1}{2} \frac{X_{6}}{X_{1}}-\left(\lambda^{2}-2(\kappa+1) \lambda\right) \frac{1}{2} \frac{X_{7}}{X_{1}}\right)\right\},
\end{aligned}
$$

where the numerical factors $X_{j}(j=1, \ldots, 14)$ and $Y_{j}(j=1,2,3,4)$ are calculated in Appendix I (see Eq.(I-26) and Eq.([-27)). The factor $x_{C}=X_{8} / 2 X_{1}=0.27591$ agrees well with the factor $x_{C}=0.27594$, calculated by Glück [73]. As a result the correlation coefficient $C_{0}$ is equal to

$$
C_{0}=-0.27591\left(A_{0}+B_{0}\right)
$$

The numerical value $C_{0}=-0.2386$, calculated at $\lambda=-1.2750$, agrees well with the experimental one $C_{0}^{\text {exp }}=$ $-0.2377(26)$ [42]. Defining the proton recoil asymmetry $C_{\exp }$ as we have defined the asymmetry $A_{\text {exp }}\left(E_{e}\right)(\operatorname{see}$ Eq. (17)) we obtain

$$
C_{\exp }=P C\left(\cos \vartheta_{1}+\cos \vartheta_{2}\right)
$$

where the polar angles $\theta_{1}$ and $\theta_{2}$ define the solid angle $\Delta \Omega_{12}=2 \pi\left(\cos \theta_{1}-\cos \theta_{2}\right)$ of the proton emission to the forward and backward hemisphere with respect to the neutron spin. 
The account for the contributions of the proton-photon correlations to the proton recoil energy and angular distribution of the radiative $\beta^{-}$-decay of the neutron [77, 78] changes the proton recoil asymmetry $C$ as follows [78] (see also Appendix I and Eq.([-33)

$$
\begin{aligned}
& C=-\left(x_{C}+\frac{\alpha}{\pi} x_{\mathrm{eff}}\right)\left(A_{0}+B_{0}\right)+\frac{1}{2} \frac{X_{9}}{X_{1}} A_{0}+\frac{1}{M} \frac{1}{1+3 \lambda^{2}}\left(\lambda \frac{1}{2} \frac{X_{12}}{X_{1}}-(\kappa+1) \lambda \frac{1}{2} \frac{X_{13}}{X_{1}}-(2 \kappa+1) \lambda \frac{1}{2} \frac{X_{14}}{X_{1}}\right. \\
& \left.-\lambda(1+\lambda) \frac{1}{2} \frac{X_{15}+Y_{3}}{X_{1}}+\lambda(1-\lambda) \frac{1}{2} \frac{X_{16}+Y_{4}}{X_{1}}\right)+\left(A_{0}+B_{0}\right) \frac{X_{8}}{X_{1}}\left\{\frac{\alpha}{\pi} \frac{1}{2} \frac{X_{2}}{X_{1}}+\frac{1}{M} \frac{1}{1+3 \lambda^{2}}\left(\frac{1}{2} \frac{X_{3}}{X_{1}}+\left(1+3 \lambda^{2}\right)\right.\right. \\
& \left.\left.\times \frac{1}{2} \frac{X_{4}+Y_{1}}{X_{1}}-\left(1-\lambda^{2}\right) \frac{1}{2} \frac{X_{5}+Y_{2}}{X_{1}}+\left(\lambda^{2}+2(\kappa+1) \lambda\right) \frac{1}{2} \frac{X_{6}}{X_{1}}-\left(\lambda^{2}-2(\kappa+1) \lambda\right) \frac{1}{2} \frac{X_{7}}{X_{1}}\right)\right\},
\end{aligned}
$$

where $x_{\text {eff }}=X_{\text {eff }} / 2 X_{1}=4.712120$ and $X_{\text {eff }}=2.215111 \mathrm{MeV}^{5}$ [78]. One may see that the contributions of the protonphoton correlations make the radiative corrections to the proton recoil asymmetry $C$ symmetric with respect to a change $A_{0} \longleftrightarrow B_{0}$ as well as the main term $C_{0}=-x_{C}\left(A_{0}+B_{0}\right)$.

Summary. The correlation coefficient $C$, describing correlations between the neutron spin and the proton $3-$ momentum, is calculated by taking into account a complete set of the $1 / M$ corrections, caused by the "weak magnetism" and the proton recoil, and the radiative corrections of order $\alpha / \pi$, calculated to leading order in the large $M$ expansion. The obtained result should be used as a theoretical background for the experimental determination of contributions of order $10^{-4}$ of interactions beyond the SM (see section [X]).

\section{STANDARD MODEL ANALYSIS OF LIFETIME OF NEUTRON}

Having integrated the electron-energy and angular distribution Eq. (6) over the directions of the electron 3momentum $\vec{k}_{e}$, the antineutrino 3 -momentum $\vec{k}$ and the electron energy $E_{e}$ within the limits $m_{e} \leq E_{e} \leq E_{0}$, we obtain the rate of the neutron $\beta^{-}$-decay. It is equal to [10]

$$
\left(\lambda_{n}\right)_{\mathrm{SM}}=\left(1+3 \lambda^{2}\right) \frac{G_{F}^{2}\left|V_{u d}\right|^{2}}{2 \pi^{3}} f_{n}\left(E_{0}, Z=1\right),
$$

where the Fermi integral $f_{n}\left(E_{0}, Z=1\right)$, given by

$$
\begin{aligned}
& f_{n}\left(E_{0}, Z=1\right)=\int_{m_{e}}^{E_{0}}\left(E_{0}-E_{e}\right)^{2} \sqrt{E_{e}^{2}-m_{e}^{2}} E_{e} F\left(E_{e}, Z=1\right)\left(1+\frac{\alpha}{\pi} g_{n}\left(E_{e}\right)\right) \\
& \times\left\{1+\frac{1}{M} \frac{1}{1+3 \lambda^{2}}\left[\left(10 \lambda^{2}-4(\kappa+1) \lambda+2\right) E_{e}-2 \lambda(\lambda-(\kappa+1))\left(E_{0}+\frac{m_{e}^{2}}{E_{e}}\right)\right]\right\} d E_{e}
\end{aligned}
$$

contains the contributions of the "weak magnetism", the proton recoil and the radiative corrections, described by the function $g_{n}\left(E_{e}\right)$. The calculation of the lifetime of the neutron with the Fermi function, determined by Eq.(5), the axial coupling constant $\lambda=-1.2750(9)$ and the CKM matrix element $V_{u d}=0.97427(15)$, gives $\left(\tau_{n}\right)_{\mathrm{SM}}=879.6(1.1) \mathrm{s}$. The error bars $\pm 1.1 \mathrm{~s}$ are defined by the error bars of the experimental value of the coupling constant and the CKM matrix element. The theoretical value of the lifetime of the neutron $\left(\tau_{n}\right)_{\mathrm{SM}}=879.6(1.1) \mathrm{s}$ agrees well with the experimental values $\tau_{n}^{(\exp )}=878.5(8) \mathrm{s}$ and $\tau_{n}^{(\exp )}=880.7(1.8) \mathrm{s}$ and $\tau_{n}^{(\exp )}=881.6(2.1) \mathrm{s}$, measured by Serebrov et al. [12], Pichlmaier et al. [79] and Arzumanov et al. [80], respectively. It agrees also well with the new world average values (w.a.v.) of the neutron lifetime $\tau_{n}^{\text {(w.a.v.) }}=880.1(1.1) \mathrm{s}[9], \tau_{n}^{\text {(w.a.v. })}=880.0(9) \mathrm{s}[81]$ and $\tau_{n}^{\text {(w.a.v.) }}=881.9(1.3) \mathrm{s}$ [82], respectively.

Summary. The lifetime of the neutron is calculated by taking into account the radiative corrections by Sirlin et al., calculated to leading order in the large $M$ expansion, and a complete set of the $1 / M$ corrections, caused by the "weak magnetism" and the proton recoil. The obtained result should be used as a theoretical background for the experimental determination of contributions of order $10^{-4}$ of interactions beyond the SM (see section IX). The numerical value of the lifetime of the neutron $\left(\tau_{n}\right)_{\mathrm{SM}}=879.6(1.1) \mathrm{s}$, calculated in this section, is left unchanged after the absorption of the Herczeg phenomenological coupling constants $a_{L L}^{h}$ and $a_{L R}^{h}$ of the left-left and left-right leptonnucleon current-current interactions (vector and axial-vector interactions beyond the SM) by the axial coupling constant $\lambda \rightarrow \lambda_{\text {eff }}=\left(\lambda-a_{L L}^{h}+a_{L R}^{h}\right) /\left(1+a_{L L}^{h}+a_{L R}^{h}\right)=\lambda-a_{L L}^{h}+a_{L R}^{h}-\lambda\left(a_{L L}^{h}+a_{L R}^{h}\right)$ and the CKM matrix element $V_{u d} \rightarrow\left(V_{u d}\right)_{\mathrm{eff}}=V_{u d}\left(1+a_{L L}^{h}+a_{L R}^{h}\right)($ see section [X) 


\section{SENSITIVITY OF ELECTRON-PROTON ENERGY DISTRIBUTION, ASYMMETRIES $A_{\exp }\left(E_{e}\right)$, $B_{\exp }\left(E_{e}\right)$ AND $C_{\exp }$ AND LIFETIME OF NEUTRON $\tau_{n}$ TO CONTRIBUTIONS OF ORDER $10^{-4}$ OF INTERACTIONS BEYOND STANDARD MODEL}

In this section we propose a theoretical analysis of the sensitivity of the electron-proton energy distribution $a\left(E_{e}, T_{p}\right)$, the proton-energy spectrum $a\left(T_{p}\right)$, the asymmetries $A_{\exp }\left(E_{e}\right), B_{\exp }\left(E_{e}\right)$ and $C_{\exp }$ and the lifetime of the neutron $\tau_{n}$ to contributions of order $10^{-4}$ of interactions beyond the SM.

The electron-proton energy distribution $a\left(E_{e}, T_{p}\right)$ and the correlation coefficients $a\left(E_{e}\right), A\left(E_{e}\right)$ and $B\left(E_{e}\right)$, including the contributions of interactions beyond the SM, can be written in the form

$$
\begin{aligned}
a\left(E_{e}, T_{p}\right) & =\zeta_{1}\left(E_{e}, T_{p}\right)_{\mathrm{eff}}+\bar{a}_{\mathrm{eff}}\left(E_{e}\right)\left(1+\frac{\alpha}{\pi} f_{n}\left(E_{e}\right)\right) \zeta_{2}\left(E_{e}, T_{p}\right) \\
a\left(E_{e}\right) & =a_{\mathrm{eff}}\left(E_{e}\right)\left(1+\frac{\alpha}{\pi} f_{n}\left(E_{e}\right)\right) \\
A\left(E_{e}\right) & =A_{\mathrm{eff}}\left(E_{e}\right)\left(1+\frac{\alpha}{\pi} f_{n}\left(E_{e}\right)\right) \\
B\left(E_{e}\right) & =B_{\mathrm{eff}}\left(E_{e}\right)
\end{aligned}
$$

where $\zeta_{1}\left(E_{e}, T_{p}\right)_{\mathrm{eff}}, \bar{a}_{\mathrm{eff}}\left(E_{e}\right), a_{\mathrm{eff}}\left(E_{e}\right), A_{\mathrm{eff}}\left(E_{e}\right)$ and $B_{\mathrm{eff}}\left(E_{e}\right)$ are the electron-proton energy distribution and the correlation coefficients, defined by the contributions of the "weak magnetism", the proton recoil and interactions beyond the SM only. They are given by

$$
\begin{aligned}
& \zeta_{1}\left(E_{e}, T_{p}\right)_{\mathrm{eff}}=\left(1-\frac{a_{4}}{a_{0}}\left(\frac{m_{e}}{E_{e}}-\left\langle\frac{m_{e}}{E_{e}}\right\rangle_{\mathrm{SM}}\right)\right) \zeta_{1}\left(E_{e}, T_{p}\right), \\
& \bar{a}_{\mathrm{eff}}\left(E_{e}\right)=\left(a_{0}\right)_{\mathrm{eff}}+\frac{1}{M} \frac{1}{1+3 \lambda^{2}} E_{0}+a_{4}\left\langle\frac{m_{e}}{E_{e}}\right\rangle_{\mathrm{SM}}, \\
& a_{\mathrm{eff}}\left(E_{e}\right)=\left(a_{0}\right)_{\mathrm{eff}}+\frac{1}{M} \frac{1}{\left(1+3 \lambda^{2}\right)^{2}}\left(a_{1} E_{0}+a_{2} E_{e}+a_{3} \frac{m_{e}^{2}}{E_{e}}\right)+a_{4} \frac{m_{e}}{E_{e}} \\
& A_{\mathrm{eff}}\left(E_{e}\right)=\left(A_{0}\right)_{\mathrm{eff}}+\frac{1}{M} \frac{1}{\left(1+3 \lambda^{2}\right)^{2}}\left(A_{1} E_{0}+A_{2} E_{e}+A_{3} \frac{m_{e}^{2}}{E_{e}}\right)+A_{4} \frac{m_{e}}{E_{e}} \\
& B_{\mathrm{eff}}\left(E_{e}\right)=\left(B_{0}\right)_{\mathrm{eff}}+\frac{1}{M} \frac{1}{\left(1+3 \lambda^{2}\right)^{2}}\left(B_{1} E_{0}+B_{2} E_{e}+B_{3} \frac{m_{e}^{2}}{E_{e}}\right)+B_{4} \frac{m_{e}}{E_{e}}
\end{aligned}
$$

where $\left\langle m_{e} / E_{e}\right\rangle_{\mathrm{SM}}$ is the average value, calculated in the SM with the electron-energy spectrum density Eq. (D-59). The terms, proportional to $\left\langle m_{e} / E_{e}\right\rangle_{\mathrm{SM}}$, come from the lifetime of the neutron, calculated at the account for the contributions of interactions beyond the SM (see Appendix G). The coefficients $a_{j}, A_{j}$ and $B_{j}$ for $j=1,2,3$ are given in Eq.(11), Eq.(12) and Eq.(13), respectively. They are calculated to next-to-leading order in the large $M$ expansion and include the contributions of the "weak magnetism" and the proton recoil only. The numerical values of these corrections are estimated in Eq.(14) at $\lambda=-1.2750$. The terms $\left(a_{0}\right)_{\text {eff }},\left(A_{0}\right)_{\text {eff }}$ and $\left(B_{0}\right)_{\text {eff }}$ are the sums of the correlation coefficients $a_{0}, A_{0}$ and $B_{0}$ and energy independent contributions of interactions beyond the SM (see Appendix G). They read

$$
\begin{aligned}
& \left(a_{0}\right)_{\mathrm{eff}}=a_{0}+\frac{1}{\left(1+3 \lambda^{2}\right)^{2}}\left(4 \lambda^{2} \operatorname{Re}\left(\delta C_{V}-\delta \bar{C}_{V}\right)+4 \lambda \operatorname{Re}\left(\delta C_{A}-\delta \bar{C}_{A}\right)\right), \\
& \left(A_{0}\right)_{\mathrm{eff}}=A_{0}+\frac{1}{\left(1+3 \lambda^{2}\right)^{2}}\left(-\lambda\left(3 \lambda^{2}-2 \lambda-1\right) \operatorname{Re}\left(\delta C_{V}-\delta \bar{C}_{V}\right)-\left(3 \lambda^{2}-2 \lambda-1\right) \operatorname{Re}\left(\delta C_{A}-\delta \bar{C}_{A}\right)\right), \\
& \left(B_{0}\right)_{\mathrm{eff}}=B_{0}+\frac{1}{\left(1+3 \lambda^{2}\right)^{2}}\left(-\lambda\left(3 \lambda^{2}+2 \lambda-1\right) \operatorname{Re}\left(\delta C_{V}-\delta \bar{C}_{V}\right)-\left(3 \lambda^{2}+2 \lambda-1\right) \operatorname{Re}\left(\delta C_{A}-\delta \bar{C}_{A}\right)\right) .
\end{aligned}
$$

The coefficients $a_{4}, A_{4}$ and $B_{4}$ are induced by interactions beyond the SM only. They are equal to

$$
\begin{aligned}
& a_{4}=-\frac{1-\lambda^{2}}{\left(1+3 \lambda^{2}\right)^{2}}\left(\operatorname{Re}\left(C_{S}-\bar{C}_{S}\right)+3 \lambda \operatorname{Re}\left(C_{T}-\bar{C}_{T}\right)\right) \\
& A_{4}=2 \frac{\lambda(1+\lambda)}{\left(1+3 \lambda^{2}\right)^{2}}\left(\operatorname{Re}\left(C_{S}-\bar{C}_{S}\right)+3 \lambda \operatorname{Re}\left(C_{T}-\bar{C}_{T}\right)\right) \\
& B_{4}=\frac{(1+\lambda)(1-3 \lambda)}{\left(1+3 \lambda^{2}\right)^{2}}\left(\lambda \operatorname{Re}\left(C_{S}-\bar{C}_{S}\right)-\operatorname{Re}\left(C_{T}-\bar{C}_{T}\right)\right) .
\end{aligned}
$$


For the derivation of Eqs. (44) and (45) we have used Eq. G-11) and Eq. (G-12 in Appendix G with the coupling constants $C_{V}=1+\delta C_{V}, \bar{C}_{V}=-1+\delta \bar{C}_{V}, C_{A}=-\lambda+\delta C_{A}$ and $\bar{C}_{A}=\lambda+\delta \bar{C}_{A}$ and have kept only the linear contributions of the deviations from the coupling constants of the SM. By assumption the axial coupling constant $\lambda$ in Eqs. (44) and (45) is determined by all interactions within the SM only.

The differences of the phenomenological coupling constants $\delta C_{V}-\delta \bar{C}_{V}, \delta C_{A}-\delta \bar{C}_{A}, C_{S}-\bar{C}_{S}$ and $C_{T}-\bar{C}_{T}$, calculated in terms of the Herczeg phenomenological lepton-nucleon coupling constants [6] (see Appendix G), take the form

$$
\begin{aligned}
\delta C_{V}-\delta \bar{C}_{V} & =2\left(a_{L L}^{h}+a_{L R}^{h}\right), \\
\delta C_{A}-\delta \bar{C}_{A} & =2\left(a_{L L}^{h}-a_{L R}^{h}\right), \\
C_{S}-\bar{C}_{S} & =2\left(A_{L L}^{h}+A_{L R}^{h}\right)=4 a_{S}, \\
C_{T}-\bar{C}_{T} & =4 \alpha_{L L}^{h}=4 a_{T} .
\end{aligned}
$$

This means that the phenomenological coupling constants $\delta C_{V}-\delta \bar{C}_{V}, \delta C_{A}-\delta \bar{C}_{A}, C_{S}-\bar{C}_{S}$ and $C_{T}-\bar{C}_{T}$ describe interactions beyond the SM of left-handed leptonic currents and left(right)-handed hadronic currents, i.e. $L \otimes L$ and $L \otimes R$, respectively.

The experimental determination of the correlation coefficients $a_{0}, A_{0}$ and $B_{0}$ runs as follows. Using the theoretical expressions for the electron-proton energy distribution $a\left(E_{e}, T_{p}\right)$ and the asymmetries $A_{\exp }\left(E_{e}\right)$ and $B_{\exp }\left(E_{e}\right)$ the experimental data on them are being fitted by a tuning of the axial coupling constant [1]. Such a procedure gives the axial-coupling constants $\lambda_{a}, \lambda_{A}$ and $\lambda_{B}$, obtained from the fit of the experimental data on $a\left(E_{e}, T_{p}\right), A_{\exp }\left(E_{e}\right)$ and $B_{\exp }\left(E_{e}\right)$, respectively. In terms of these axial coupling constants one may define the correlation coefficients $\left(a_{0}\right)_{\mathrm{eff}}^{(\exp )}$, $\left(A_{0}\right)_{\mathrm{eff}}^{(\exp )}$ and $\left(B_{0}\right)_{\mathrm{eff}}^{(\exp )}$, respectively, as follows

$$
\left(a_{0}\right)_{\mathrm{eff}}^{(\exp )}=\frac{1-\lambda_{a}^{2}}{1+3 \lambda_{a}^{2}} \quad, \quad\left(A_{0}\right)_{\mathrm{eff}}^{(\exp )}=-2 \frac{\lambda_{A}\left(1+\lambda_{A}\right)}{1+3 \lambda_{A}^{2}} \quad, \quad\left(B_{0}\right)_{\mathrm{eff}}^{(\exp )}=-2 \frac{\lambda_{B}\left(1-\lambda_{B}\right)}{1+3 \lambda_{B}^{2}}
$$

The theoretical expressions for the axial coupling constants $\lambda_{a}, \lambda_{A}$ and $\lambda_{B}$ in terms of the axial coupling constant $\lambda$, defined by interactions within the SM only, and the contributions of interactions beyond the SM are

$$
\begin{aligned}
& \lambda_{a}=\lambda-\frac{1}{2}\left(\lambda \operatorname{Re}\left(\delta C_{V}-\delta \bar{C}_{V}\right)+\operatorname{Re}\left(\delta C_{A}-\delta \bar{C}_{A}\right)\right), \\
& \lambda_{A}=\lambda-\frac{1}{2}\left(\lambda \operatorname{Re}\left(\delta C_{V}-\delta \bar{C}_{V}\right)+\operatorname{Re}\left(\delta C_{A}-\delta \bar{C}_{A}\right)\right), \\
& \lambda_{B}=\lambda-\frac{1}{2}\left(\lambda \operatorname{Re}\left(\delta C_{V}-\delta \bar{C}_{V}\right)+\operatorname{Re}\left(\delta C_{A}-\delta \bar{C}_{A}\right)\right) .
\end{aligned}
$$

Thus, in the linear approximation with respect to the deviations of the phenomenological coupling constants of interactions beyond the SM from the coupling constants of the SM we obtain that $\lambda_{a}=\lambda_{A}=\lambda_{B}$. This agrees well with the results obtained in [43]- 45]. Replacing $\lambda_{a}, \lambda_{A}$ and $\lambda_{B}$ by $\lambda_{\text {eff }}$, which includes the contributions of vector and axial-vector interactions beyond the SM in addition to the contributions of interactions within the SM, and denoting

$$
b_{F}=\frac{1}{1+3 \lambda_{\text {eff }}^{2}}\left(\operatorname{Re}\left(C_{S}-\bar{C}_{S}\right)+3 \lambda_{\text {eff }} \operatorname{Re}\left(C_{T}-\bar{C}_{T}\right)\right)=\frac{4}{1+3 \lambda_{\text {eff }}^{2}}\left(\operatorname{Re}\left(a_{S}\right)+3 \lambda_{\text {eff }} \operatorname{Re}\left(a_{T}\right)\right)
$$

and

$$
c_{S T}=\frac{1}{1+3 \lambda_{\mathrm{eff}}^{2}}\left(\lambda_{\mathrm{eff}} \operatorname{Re}\left(C_{S}-\bar{C}_{S}\right)-\operatorname{Re}\left(C_{T}-\bar{C}_{T}\right)\right)=\frac{4}{1+3 \lambda_{\mathrm{eff}}^{2}}\left(\lambda_{\mathrm{eff}} \operatorname{Re}\left(a_{S}\right)-\operatorname{Re}\left(a_{T}\right)\right),
$$

where $b_{F}$ is the Fierz term (see Appendix $\mathrm{G}$ ), we obtain the electron-proton energy distribution $a\left(E_{e}, T_{p}\right)$ and the correlation coefficients $a\left(E_{e}\right), A\left(E_{e}\right)$ and $B\left(E_{e}\right)$ in the following form

$$
\begin{aligned}
a\left(E_{e}, T_{p}\right) & =\left(1-b_{F}\left\langle\frac{m_{e}}{E_{e}}\right\rangle_{\mathrm{SM}}\right)\left\{\left(1+b_{F} \frac{m_{e}}{E_{e}}\right) \zeta_{1}\left(E_{e}, T_{p}\right)+a_{0}\left(1+\frac{1}{1-\lambda_{\text {eff }}^{2}} \frac{E_{0}}{M}\right)\left(1+\frac{\alpha}{\pi} f_{n}\left(E_{e}\right)\right) \zeta_{2}\left(E_{e}, T_{p}\right)\right\}, \\
a\left(E_{e}\right) & =a_{0}\left(1-b_{F} \frac{m_{e}}{E_{e}}\right)\left(1+\frac{\alpha}{\pi} f_{n}\left(E_{e}\right)\right)\left\{1+\frac{1}{M} \frac{1}{\left(1-\lambda_{\text {eff }}^{2}\right)\left(1+3 \lambda_{\text {eff }}^{2}\right)}\left(a_{1} E_{0}+a_{2} E_{e}+a_{3} \frac{m_{e}^{2}}{E_{e}}\right)\right\}, \\
A\left(E_{e}\right) & =A_{0}\left(1-b_{F} \frac{m_{e}}{E_{e}}\right)\left(1+\frac{\alpha}{\pi} f_{n}\left(E_{e}\right)\right)\left\{1-\frac{1}{M} \frac{1}{2 \lambda_{\text {eff }}\left(1+\lambda_{\text {eff }}\right)\left(1+3 \lambda_{\text {eff }}^{2}\right)}\left(A_{1} E_{0}+A_{2} E_{e}+A_{3} \frac{m_{e}^{2}}{E_{e}}\right)\right\} \\
B\left(E_{e}\right) & =B_{0}\left(1-\frac{\left(1+\lambda_{\text {eff }}\right)\left(1-3 \lambda_{\text {eff }}\right)}{2 \lambda_{\text {eff }}\left(1-\lambda_{\text {eff }}\right)} c_{S T} \frac{m_{e}}{E_{e}}\right)\left\{1-\frac{1}{M} \frac{1}{2 \lambda_{\text {eff }}\left(1-\lambda_{\text {eff }}\right)\left(1+3 \lambda_{\text {eff }}^{2}\right)}\left(B_{1} E_{0}+B_{2} E_{e}+B_{3} \frac{m_{e}^{2}}{E_{e}}\right)\right\},(51
\end{aligned}
$$


where the coefficients $a_{j}, A_{j}$ and $B_{j}$ for $j=1,2,3$ are defined in Eq.(11), Eq.(12) and Eq. (13), respectively, with the replacement $\lambda \rightarrow \lambda_{\text {eff }}$. The same replacement defines the correlation coefficients $a_{0}, A_{0}$ and $B_{0}$ in terms of $\lambda_{\text {eff }}$ (see Eq.(8) $)$.

The correlation coefficients $a\left(E_{e}\right), A\left(E_{e}\right)$ and $B\left(E_{e}\right)$, extended by the contributions of interactions beyond the SM, together with the correlation coefficients $K_{n}\left(E_{e}\right)$ and $Q_{n}\left(E_{e}\right)$, caused by the contributions of the $1 / M$ corrections from the "weak magnetism" and the proton recoil only, determine the asymmetries $A_{\exp }\left(E_{e}\right)$ and $B_{\exp }\left(E_{e}\right)$. For the calculation of the electron asymmetry $A_{\exp }\left(E_{e}\right)$, taking into account the contributions of interactions beyond the SM, we have to replace the correlation coefficient $A^{(\mathrm{W})}\left(E_{e}\right)$ in Eq. (17) by the expression

$$
A^{(\mathrm{W})}\left(E_{e}\right)=A_{0}\left(1-b_{F} \frac{m_{e}}{E_{e}}\right)\left\{1-\frac{1}{M} \frac{1}{2 \lambda_{\text {eff }}\left(1+\lambda_{\text {eff }}\right)\left(1+3 \lambda_{\text {eff }}^{2}\right)}\left(A_{1}^{(\mathrm{W})} E_{0}+A_{2}^{(\mathrm{W})} E_{e}+A_{3}^{(\mathrm{W})} \frac{m_{e}^{2}}{E_{e}}\right)\right\},
$$

where the coefficients $A_{j}^{(\mathrm{W})}$ are given in Eq.(20) with the replacement $\lambda \rightarrow \lambda_{\text {eff }}$.

The antineutrino asymmetry $B_{\exp }\left(E_{e}\right)$ is defined by Eq.(27) and Eq.(28) for $r \leq 1$ and $r \geq 1$, respectively. For the account for the contributions of interactions beyond the SM the correlation coefficients $a\left(E_{e}\right), A\left(E_{e}\right)$ and $B\left(E_{e}\right)$ should be taken in the form, given by Eq. (51).

The proton recoil asymmetry $C_{\text {exp }}$ completes the set of asymmetries, which can be measured in the neutron $\beta^{--}$ decay with a polarized neutron and unpolarised proton and electron. The correlation coefficient $C_{\text {eff }}$ (see Appendix I), defining the asymmetry $C_{\exp }$ and extended by the contributions of interactions beyond the SM, takes the form

$$
C_{\text {eff }}=-x_{C}\left(A_{0}+B_{0}\right)+\frac{\lambda_{\text {eff }}\left(1+\lambda_{\text {eff }}\right)}{1+3 \lambda_{\text {eff }}^{2}} b_{F} \frac{X_{17}}{X_{1}}-\frac{1}{2} \frac{\left(1+\lambda_{\text {eff }}\right)\left(1-3 \lambda_{\text {eff }}\right)}{1+3 \lambda_{\text {eff }}^{2}} c_{S T} \frac{X_{18}}{X_{1}}+C_{\mathrm{SM}},
$$

where $C_{\mathrm{SM}}=C+x_{C}\left(A_{0}+B_{0}\right)$ and $x_{C}=0.27591$ (see Eq.(39) and Eq.(37)). The numerical factors $X_{17} / X_{1}=-0.90187$ and $X_{18} / X_{1}=0.39806$ are calculated in Appendix I (see Eq. [-26) ).

Using the results, obtained in Appendix G, the theoretical expression for the rate of the neutron $\beta^{-}-$decay, including the contributions of interactions beyond the SM, is

$$
\begin{aligned}
& \lambda_{n}=\left(\lambda_{n}\right)_{\mathrm{SM}}\left(1+\frac{1}{1+3 \lambda^{2}}\left(\operatorname{Re}\left(\delta C_{V}-\delta \bar{C}_{V}\right)-3 \lambda \operatorname{Re}\left(\delta C_{A}-\delta \bar{C}_{A}\right)\right)+b_{F}\left\langle\frac{m_{e}}{E_{e}}\right\rangle_{\mathrm{SM}}\right)= \\
& =\frac{G_{F}^{2}\left|V_{u d}\right|^{2}}{2 \pi^{3}} f_{n}\left(E_{0}, Z=1\right)\left(1+3 \lambda^{2}\right)\left(1+\frac{1}{1+3 \lambda^{2}}\left(\operatorname{Re}\left(\delta C_{V}-\delta \bar{C}_{V}\right)-3 \lambda \operatorname{Re}\left(\delta C_{A}-\delta \bar{C}_{A}\right)\right)+b_{F}\left\langle\frac{m_{e}}{E_{e}}\right\rangle_{\mathrm{SM}}\right),
\end{aligned}
$$

where $\left(\lambda_{n}\right)_{\mathrm{SM}}$ is the lifetime of the neutron, calculated within the SM (see Eq.(40) and Eq. (411)), and $\left\langle m_{e} / E_{e}\right\rangle_{\mathrm{SM}}$ is the average value, calculated with the electron-energy spectrum density Eq.(D-59). Now we have to define the rate of the neutron $\beta^{-}$-decay Eq.(54) in terms of the axial coupling constant $\lambda_{\text {eff, }}$ which is related to the axial coupling constant $\lambda$ as

$$
\lambda=\lambda_{\text {eff }}+\frac{1}{2}\left(\lambda_{\text {eff }} \operatorname{Re}\left(\delta C_{V}-\delta \bar{C}_{V}\right)+\operatorname{Re}\left(\delta C_{A}-\delta \bar{C}_{A}\right)\right)
$$

Substituting Eq.(55) into Eq.(54) and keeping only the linear terms in power of $\operatorname{Re}\left(\delta C_{V}-\delta \bar{C}_{V}\right)$ and $\operatorname{Re}\left(\delta C_{A}-\delta \bar{C}_{A}\right)$ one may show that

$$
\begin{aligned}
& \left(1+3 \lambda^{2}\right)\left(1+\frac{1}{1+3 \lambda^{2}}\left(\operatorname{Re}\left(\delta C_{V}-\delta \bar{C}_{V}\right)-3 \lambda \operatorname{Re}\left(\delta C_{A}-\delta \bar{C}_{A}\right)\right)+b_{F}\left\langle\frac{m_{e}}{E_{e}}\right\rangle_{\mathrm{SM}}\right)= \\
& =\left(1+\operatorname{Re}\left(\delta C_{V}-\delta \bar{C}_{V}\right)\right)\left(1+3 \lambda_{\text {eff }}^{2}\right)\left(1+b_{F}\left\langle\frac{m_{e}}{E_{e}}\right\rangle_{\mathrm{SM}}\right) .
\end{aligned}
$$

This gives the rate of the neutron $\beta^{-}$-decay equal to

$$
\lambda_{n}=\frac{G_{F}^{2}\left|V_{u d}\right|^{2}}{2 \pi^{3}} f_{n}\left(E_{0}, Z=1\right)\left(1+\operatorname{Re}\left(\delta C_{V}-\delta \bar{C}_{V}\right)\right)\left(1+3 \lambda_{\mathrm{eff}}^{2}\right)\left(1+b_{F}\left\langle\frac{m_{e}}{E_{e}}\right\rangle_{\mathrm{SM}}\right),
$$

where the Fermi integral is given by Eq.(41) with the replacement $\lambda \rightarrow \lambda_{\text {eff }}$.

If we introduce again $\left(\lambda_{n}\right)_{\mathrm{SM}}$, defined by Eq.(40) and Eq.410 with the replacement $\lambda \rightarrow \lambda_{\text {eff }}$, we get the following expression for the rate of the neutron $\beta^{-}-$decay, corrected by the contributions of interactions beyond the SM taken to linear approximation with respect to the Herczeg phenomenological coupling constants

$$
\lambda_{n}=\left(\lambda_{n}\right)_{\mathrm{SM}}\left(1+2 \operatorname{Re}\left(a_{L L}^{h}+a_{L R}^{h}\right)\right)\left(1+b_{F}\left\langle\frac{m_{e}}{E_{e}}\right\rangle_{\mathrm{SM}}\right)
$$


where we have set $\operatorname{Re}\left(\delta C_{V}-\delta \bar{C}_{V}\right)=2 \operatorname{Re}\left(a_{L L}^{h}+a_{L R}^{h}\right)$. Thus, at first glimpse a deviation of the experimental values $\tau_{n}=1 / \lambda_{n}$ of the lifetime of the neutron considered relative to the theoretical value of the lifetime of the neutron $\left(\tau_{n}\right)_{\mathrm{SM}}=1 /\left(\lambda_{n}\right)_{\mathrm{SM}}$, calculated in the SM at zero Herczeg coupling constants, may give an information about the contribution of the Herczeg left-left and left-right lepton-nucleon current-current interactions (vector and axial-vector interactions beyond the SM) by using the experimental value of the Fierz term $b_{F}$, determined from the experimental data on the electron-proton energy distribution $a\left(E_{e}, T_{p}\right)$, the proton-energy spectrum $a\left(T_{p}\right)$ and the asymmetries $A_{\exp }\left(E_{e}\right), B_{\exp }\left(E_{e}\right)$ and $C_{\text {exp }}$. However, the problem is that the Herczeg phenomenological interactions beyond the $\mathrm{SM}$, if they exist, should exist always together with the interactions of the SM, and a separation of these interactions is rather artificial.

Hence, using the Hamilton Eq. (G-1) and Eq. G-2 we may redefine the axial coupling constant and the CKM matrix element as follows $\lambda_{\mathrm{eff}}=\left(\lambda-a_{L L}^{h}+a_{L R}^{h}\right) /\left(1+a_{L L}^{h}+a_{L R}^{h}\right)$ and $\left(V_{u d}\right)_{\mathrm{eff}}=V_{u d}\left(1+a_{L L}^{h}+a_{L R}^{h}\right)$ as it has been proposed in 43] [45]. After such a change one may show that the rate of the neutron $\beta^{-}$-decay may contain only the contribution of the Fierz term

$$
\lambda_{n}=\left(\lambda_{n}\right)_{\mathrm{SM}}\left(1+b_{F}\left\langle\frac{m_{e}}{E_{e}}\right\rangle_{\mathrm{SM}}\right)
$$

where $\left(\lambda_{n}\right)_{\mathrm{SM}}$ is given by Eq.400 and Eq. (41) with the replacement $\lambda \rightarrow \lambda_{\text {eff }}$ and $V_{u d} \rightarrow\left(V_{u d}\right)_{\mathrm{eff}}$.

The definition of the effective coupling constant $\lambda_{\text {eff }}=\left(\lambda-a_{L L}^{h}+a_{L R}^{h}\right) /\left(1+a_{L L}^{h}+a_{L R}^{h}\right)$ and the CKM matrix element $\left(V_{u d}\right)_{\text {eff }}=V_{u d}\left(1+a_{L L}^{h}+a_{L R}^{h}\right)$ at the Hamiltonian level introduces the imaginary parts to the axial coupling constant and the CKM matrix element

$$
\begin{aligned}
\lambda_{\mathrm{eff}} & =\operatorname{Re} \lambda_{\mathrm{eff}}+i \operatorname{Im} \lambda_{\mathrm{eff}}, \\
\left(V_{u d}\right)_{\mathrm{eff}} & =V_{u d}\left(1+\operatorname{Re}\left(a_{L L}^{h}+a_{L R}^{h}\right)\right)\left(1+i \operatorname{Im}\left(a_{L L}^{h}+a_{L R}^{h}\right)\right)
\end{aligned}
$$

where $\operatorname{Im} \lambda_{\text {eff }}$ is equal to

$$
\operatorname{Im} \lambda_{\text {eff }}=-\left(1+\operatorname{Re} \lambda_{\text {eff }}\right) \operatorname{Im}\left(a_{L L}^{h}\right)+\left(1-\operatorname{Re} \lambda_{\text {eff }}\right) \operatorname{Im}\left(a_{L R}^{h}\right) .
$$

An information about the imaginary part of the axial coupling constant $\lambda_{\text {eff }}$ one may obtain by measuring the correlation coefficient $D\left(E_{e}\right)$, describing a violation of time reversal invariance. From Eq. (G-11), Eq. (G-12) and Eq.61) we obtain

$$
\begin{aligned}
& D\left(E_{e}\right)=D_{\mathrm{SM}}\left(E_{e}\right)-\frac{2}{1+3 \lambda_{\text {eff }}^{2}}\left(\lambda_{\text {eff }} \operatorname{Im}\left(a_{L L}^{h}+a_{L R}^{h}\right)+\operatorname{Im}\left(a_{L L}^{h}-a_{L R}^{h}\right)\right)= \\
& =D_{\mathrm{SM}}\left(E_{e}\right)-\frac{2}{1+3 \lambda_{\text {eff }}^{2}}\left(\left(1+\lambda_{\text {eff }}\right) \operatorname{Im}\left(a_{L L}^{h}\right)-\left(1-\lambda_{\text {eff }}\right) \operatorname{Im}\left(a_{L R}^{h}\right)\right)=D_{\mathrm{SM}}\left(E_{e}\right)+\frac{2 \operatorname{Im} \lambda_{\text {eff }}}{1+3 \lambda_{\text {eff }}^{2}},
\end{aligned}
$$

where we have replaced $\operatorname{Re} \lambda_{\text {eff }}$ by $\lambda_{\text {eff }}$, having neglected the contribution of the imaginary part, and $D_{\mathrm{SM}}\left(E_{e}\right)$ is the contribution to the correlation coefficient $D\left(E_{e}\right)$, calculated within the SM [54]-61]. As we have estimated in section III such a contribution, caused by the electron-proton interaction in the final state [54]-57, 61, is of order $10^{-5}$ for the electron kinetic energies $250 \mathrm{keV} \leq T_{e} \leq 455 \mathrm{keV}$. Thus, the correlation coefficient $D\left(E_{e}\right)$, defined in the same energy region, should be sensitive to the contributions beyond the SM of $10^{-4}$. Recently the experimental value $D_{\exp }\left(E_{e}\right)=(-4 \pm 6) \times 10^{-4}$ of the correlation coefficient $D\left(E_{e}\right)$ [1, 9] has been substantially improved with a result $D_{\exp }\left(E_{e}\right)=\left(-0.96 \pm 1.89_{\text {stat }} \pm 1.01_{\text {syst }}\right) \times 10^{-4}[83$. However, the new experimental value as well as the old one still implies that to order $10^{-4}$ the correlation coefficient $D\left(E_{e}\right)$ is commensurable with zero. Hence, to order $10^{-4}$ the imaginary part of the axial coupling constant $\lambda_{\text {eff }}$ is also commensurable with zero, i.e. $\operatorname{Im} \lambda_{\text {eff }}=0$. Nevertheless, setting $\lambda_{\text {eff }}=\operatorname{Re} \lambda_{\text {eff }}=-1.2750$ and using the relation $\operatorname{Im} \lambda_{\text {eff }}=0$ we may obtain that $\operatorname{Im}\left(a_{L R}^{h}\right)=-0.12 \operatorname{Im}\left(a_{L L}^{h}\right)$. This adds an additional phase shift $e^{i 0.88 \operatorname{Im}\left(a_{L L}^{h}\right)}$ to the CKM matrix element $\left(V_{u d}\right)_{\mathrm{eff}}$.

The axial coupling constant $\lambda_{a}$ and the correlation coefficient $a_{0}$ may be also determined by measuring the protonenergy spectrum Eq.(33) (see [40]). The proton-energy spectrum, taking into account the contributions of interactions beyond the SM, is

$$
a_{\mathrm{eff}}\left(T_{p}\right)=\left(1-b_{F}\left\langle\frac{m_{e}}{E_{e}}\right\rangle_{\mathrm{SM}}\right)\left\{g_{1}\left(T_{p}\right)_{\mathrm{eff}}+a_{0}\left(1+\frac{1}{1-\lambda^{2}} \frac{E_{0}}{M}\right) g_{2}\left(T_{p}\right)\right\},
$$

where the functions $g_{1}\left(T_{p}\right)_{\text {eff }}$ and $g_{2}\left(T_{p}\right)$ are defined by the integrals

$$
\begin{aligned}
& g_{1}\left(T_{p}\right)_{\mathrm{eff}}=\int_{\left(E_{e}\right)_{\min }}^{\left(E_{e}\right)_{\max }}\left(1+b_{F} \frac{m_{e}}{E_{e}}\right)\left(1+\frac{\alpha}{\pi} g_{n}\left(E_{e}\right)\right) \zeta_{1}\left(E_{e}, T_{p}\right) F\left(E_{e}, Z=1\right) E_{e} d E_{e}, \\
& g_{2}\left(T_{p}\right)=\int_{\left(E_{e}\right)_{\min }}^{\left(E_{e}\right)_{\max }}\left(1+\frac{\alpha}{\pi} g_{n}\left(E_{e}\right)+\frac{\alpha}{\pi} f_{n}\left(E_{e}\right)\right) \zeta_{2}\left(E_{e}, T_{p}\right) F\left(E_{e}, Z=1\right) E_{e} d E_{e},
\end{aligned}
$$


where the limits of integration $\left(E_{e}\right)_{\max / \min }$ are given in Appendix I.

Summary. We have analysed the sensitivity of the electron-proton energy distribution $a\left(E_{e}, T_{p}\right)$, the proton-energy spectrum $a\left(T_{p}\right)$, the asymmetries $A_{\exp }\left(E_{e}\right), B_{\exp }\left(E_{e}\right), C_{\exp }$ and the lifetime of the neutron $\tau_{n}$ to contributions of order $10^{-4}$ of interactions beyond the SM, taken to linear approximation with respect to the Herczeg phenomenological coupling constants of weak lepton-nucleon current-current interactions. We have shown that in such an approximation the axial coupling constant $\lambda_{\text {eff }}$ and the CKM matrix element $\left(V_{u d}\right)_{\text {eff }}$ absorb the contributions of the Herczeg leftleft and left-right lepton-nucleon current-current interactions with the coupling constants $a_{L L}^{h}$ and $a_{L R}^{h}$. In this approximation the axial coupling constant does not acquire an imaginary part to order $10^{-4}$, but the CKM matrix element becomes an additional phase $e^{i \operatorname{Im}\left(a_{L L}^{h}+a_{L R}^{h}\right)}$. Thus, after the measurements of the electron-proton energy spectrum $a\left(E_{e}, T_{p}\right)$, the proton-energy spectrum $a\left(T_{p}\right)$, the asymmetries $A_{\exp }\left(E_{e}\right), B_{\exp }\left(E_{e}\right)$ and $C_{\exp }$ and the lifetime of the neutron $\tau_{n}$ one may determine the axial coupling constant $\lambda_{\text {eff }}$ and the real parts of the scalar and tensor coupling constants $a_{S}$ and $a_{T}$, defined in Eq.(46),

$$
\begin{aligned}
\operatorname{Re}\left(a_{S}\right)+3 \lambda_{\text {eff }} \operatorname{Re}\left(a_{T}\right) & =\frac{1+3 \lambda_{\text {eff }}^{2}}{4} b_{F} \\
\lambda_{\text {eff }} \operatorname{Re}\left(a_{S}\right)-\operatorname{Re}\left(a_{T}\right) & =\frac{1+3 \lambda_{\text {eff }}^{2}}{4} c_{S T},
\end{aligned}
$$

where in the r.h.s. of the algebraical equations $\lambda_{\text {eff }}, b_{F}$ and $c_{S T}$ are the experimental values with their experimental errors.

\section{CONCLUSION}

We have analysed the sensitivity of the electron-proton energy distribution $a\left(E_{e}, T_{p}\right)$, the proton-energy spectrum $a\left(T_{p}\right)$, and the asymmetries $A_{\exp }\left(E_{e}\right), B_{\exp }\left(E_{e}\right)$ and $C_{\exp }$ of the correlations between the neutron spin and 3-momenta of the decay electron, antineutrino and proton, respectively, for the neutron $\beta^{-}$-decay with a polarized neutron and unpolarised proton and electron to contributions of order $10^{-4}$ of interactions beyond the SM. For the analysis of contributions of order $10^{-4}$ we have used the linear approximation for the correlation coefficient with respect to the Herczeg phenomenological coupling constants of weak lepton-nucleon current-current interactions. We have shown that in such an approximation the Herczeg right-left and right-right lepton-nucleon current-current interactions with the coupling constants $a_{R L}^{h}$ and $a_{R R}^{h}$ give no contributions to the correlation coefficients of the neutron $\beta^{-}-$decay and the lifetime of the neutron. Then, the contributions of the Herczeg left-left and left-right lepton-nucleon currentcurrent interactions with the coupling constants $a_{L L}^{h}$ and $a_{L R}^{h}$ may be absorbed by the axial coupling constant, which we denote as $\lambda_{\text {eff }}=\lambda-\operatorname{Re}\left(a_{L L}^{h}-a_{L R}^{h}\right)+\lambda \operatorname{Re}\left(a_{L L}^{h}+a_{L R}^{h}\right)$ and the CKM matrix element $\left(V_{u d}\right)_{\text {eff }}=V_{u d}\left(1+\operatorname{Re}\left(a_{L L}^{h}+a_{L R}^{h}\right)\right)$. We have shown that the Herczeg coupling constants $a_{L L}^{h}$ and $a_{L R}^{h}$ in the effective Hamiltonian of weak interactions may be absorbed by the axial coupling constant $\lambda_{\text {eff }}=\left(\lambda-a_{L L}^{h}+a_{L R}^{h}\right) /\left(1+a_{L L}^{h}+a_{L R}^{h}\right)$ and the CKM matrix element $\left(V_{u d}\right)_{\mathrm{eff}}=V_{u d}\left(1+a_{L L}^{h}+a_{L R}^{h}\right)$. Using the experimental data on the correlation coefficient $D\left(E_{e}\right)$ we have shown that at the level of $10^{-4}$ the imaginary part of the axial coupling constant $\lambda_{\text {eff }}$ is equal to zero, whereas the CKM matrix element acquires an additional phase $e^{i \operatorname{Im}\left(a_{L L}^{h}+a_{L R}^{h}\right)}$. This shows that in addition to the background, calculated in the SM, the correlation coefficients of the neutron $\beta^{-}$-decay and the lifetime of the neutron, calculated to linear approximation with respect to the Herczeg coupling constants of lepton-nucleon current-current interactions, depend on the contributions of the scalar and tensor interactions only. This agrees with recent results, obtained in [43]- 45].

We have shown that the contributions of the scalar and tensor interactions beyond the SM are described by the Fierz term $b_{F}$ and the coupling constant $c_{S T}$. The Fierz term may be determined from the experimental data on the asymmetry $A_{\exp }\left(E_{e}\right)$ and the electron-proton energy distribution $a\left(E_{e}, T_{p}\right)$ (or the proton-energy spectrum $a\left(T_{p}\right)$ ). The coupling constant $c_{S T}$ may be determined from the experimental data on the asymmetry $B_{\exp }\left(E_{e}\right)$ and the proton recoil asymmetry $C_{\text {exp. }}$. This allows to determine the scalar $\operatorname{Re}\left(a_{S}\right)$ and tensor $\operatorname{Re}\left(a_{T}\right)$ coupling constant by solving the system of algebraical equations (see Eq.(65)

$$
\begin{aligned}
\operatorname{Re}\left(a_{S}\right)+3 \lambda_{\text {eff }} \operatorname{Re}\left(a_{T}\right) & =\frac{1+3 \lambda_{\mathrm{eff}}^{2}}{4} b_{F} \\
\lambda_{\text {eff }} \operatorname{Re}\left(a_{S}\right)-\operatorname{Re}\left(a_{T}\right) & =\frac{1+3 \lambda_{\mathrm{eff}}^{2}}{4} c_{S T},
\end{aligned}
$$

The lifetime of the neutron is defined by the background, calculated in the SM, and the Fierz term (see Eq.(59))

$$
\tau_{n}=\left(\tau_{n}\right)_{\mathrm{SM}}\left(1-b_{F}\left\langle\frac{m_{e}}{E_{e}}\right\rangle_{\mathrm{SM}}\right),
$$


where $\left(\tau_{n}\right)_{\mathrm{SM}}$ and $\left\langle m_{e} / E_{e}\right\rangle_{\mathrm{SM}}$ are calculated in the SM.

We would like to note that the experimental analysis of the neutron $\beta^{-}$-decay with a polarized neutron and unpolarised proton and electron may be carried out in terms the electron-proton energy distribution $a\left(E_{e}, T_{p}\right)$, the proton-energy spectrum $a\left(T_{p}\right)$, the asymmetries $A_{\exp }\left(E_{e}\right), B_{\exp }\left(E_{e}\right), C_{\exp }$ and the lifetime of the neutron $\tau_{n}$. From the fit of the experimental data on these energy distributions, asymmetries and the lifetime we determine three parameters, i.e. the axial coupling constant $\lambda_{\text {eff }}$, the Fierz term $b_{F}$ and the coupling constant $c_{S T}$. In order to determine these parameters without correlations between them it suffices to use experimental data, obtained only in three out of five independent experiments. This means that experimental data, obtained from other two independent experiments, should be described well by the parameters $\left(\lambda_{\text {eff }}, b_{F}, c_{S T}\right)$, determined from the first three experiments. The deviations from the predicted values should be much smaller compared with $10^{-4}$, since they may be explained only by the contributions of higher powers of the Herczeg coupling constants.

We have to note that the theoretical analysis of the sensitivity of the electron-proton energy $\operatorname{distribution} a\left(E_{e}, T_{p}\right)$, the proton-energy spectrum $a\left(T_{p}\right)$, the asymmetries $A_{\exp }\left(E_{e}\right), B_{\exp }\left(E_{e}\right)$ and $C_{\exp }$ of the neutron $\beta^{-}$-decay and the lifetime of the neutron to contributions of order $10^{-4}$ of interactions beyond the SM we have carried out above the background, calculated within the SM. We have taken into account a complete set of the $1 / M$ corrections, caused by the "weak magnetism" and the proton recoil, calculated to next-to-leading order in the large $M$ or the large proton mass expansion, and the radiative corrections of order $\alpha / \pi$, calculated to leading order in the large $M$ or the large proton mass expansion.

The corrections, caused by the "weak magnetism" and the proton recoil are calculated in analytical agreement with the results, obtained by Wilkinson [34] and Gudkov et al. [32. The radiative corrections to the lifetime of the neutron and the correlation coefficients of the neutron $\beta^{-}$-decay with a polarized neutron and unpolarised decay proton and electron, calculated in this paper, we have given in terms of two functions $(\alpha / \pi) g_{n}\left(E_{e}\right)$ and $(\alpha / \pi) f_{n}\left(E_{e}\right)$. The analytical expressions of these functions are given in Eq.(D-58) of Appendix D. They are in analytical agreement with the radiative corrections, calculated in [18] [30] and in [31, 32], respectively. We have confirmed Sirlin's assertion that an unambiguous definition of the observable radiative corrections to the lifetime of the neutron is fully caused by the requirement of gauge invariance of the amplitude of one--virtual photon exchanges of the continuum-state $\beta^{-}-$decay of the neutron [18].

We have improved the theoretical expressions for the asymmetries $A_{\exp }\left(E_{e}\right), B_{\exp }\left(E_{e}\right)$ and $C_{\exp }$ with respect to the expressions, used in [1, 37], 38, 39] and [42] for the experimental determination of the axial coupling constant $\lambda=$

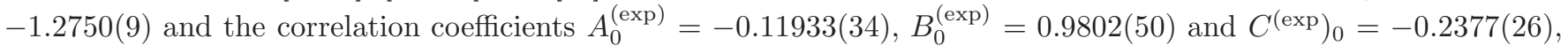
respectively. We have added the radiative corrections to the electron asymmetry $A_{\exp }\left(E_{e}\right)$ and the $1 / M$ and radiative corrections to the antineutrino $B_{\exp }\left(E_{e}\right)$ and proton $C_{\exp }$ asymmetries. In connection with the experimental analysis of contributions of order $10^{-4}$ of interactions beyond the SM to the neutron $\beta^{-}$-decay we have calculated the electronproton energy distribution $a\left(E_{e}, T_{p}\right)$ and the proton-energy spectrum $a\left(T_{p}\right)$ by taking into account the complete set of the $1 / M$ corrections, caused by the "weak magnetism" and the proton recoil, and the radiative corrections of order $\alpha / \pi$.

As has been pointed out by Glück [7], the contributions of the radiative $\beta^{-}-$decay of the neutron to the protonenergy spectrum and angular distribution demand a detailed analysis of the proton-photon correlations, which appear in the proton recoil energy and angular distribution of the radiative $\beta^{-}$-decay of the neutron. For the aim of a consistent calculation of the contributions of the nucleus-photon and hadron-photon correlations in the radiative nuclear and hadronic $\beta$-decays Glück has used the Monte Carlo simulation method. Recently the calculation of the proton-photon correlations in the radiative $\beta^{-}$-decay of the neutron has been performed in [78]. There it has been shown that the contributions of the proton-photon correlations to the lifetime of the neutron $\tau_{n}$, the proton-energy spectrum $a\left(T_{p}\right)$ and the electron-proton energy distribution $a\left(E_{e}, T_{p}\right)$ are smaller compared with the contributions of the radiative corrections, described by the functions $g_{n}\left(E_{e}\right)$ and $f_{n}\left(E_{e}\right)$. At the level of $10^{-5}$ accuracy the contributions of the proton-photon correlations to part of the proton recoil angular distribution, independent of $\cos \theta_{p}$, can be neglected. In turn, the contributions of the proton-photon correlations to the proton recoil asymmetry $C$, i.e. in part of the proton recoil angular distribution proportional to $\cos \theta_{p}$, are of order $10^{-4}$. The account for these contributions makes the radiative corrections to the proton recoil angular distribution and the proton recoil asymmetry $C$ symmetric with respect to a change $A_{0} \longleftrightarrow B_{0}$ as well as the main term $C_{0}=-x_{C}\left(A_{0}+B_{0}\right)$. A detailed analysis of the proton-energy spectrum $a\left(T_{p}\right)$ has been recently carried out in [78]. In addition to the protonenergy spectrum $a\left(T_{p}\right)$, calculated in this paper, the authors of the paper [78] have included the contributions of the proton-photon correlations and analysed the proton energy regions, convenient for measurements of the Fierz term $b_{F}$, caused by scalar and tensor interactions beyond the SM.

We have also shown that at the present level of the experimental accuracy the lifetime of the neutron is described well by the SM with the account for a complete set of the $1 / M$ corrections, caused by the "weak magnetism" and the proton recoil, calculated to next-to-leading order in the large proton mass expansion, and the radiative corrections of order $\alpha / \pi$, calculated to leading order in the large proton mass expansion. The theoretical value 
of the lifetime of the neutron $\left(\tau_{n}\right)_{\mathrm{SM}}=879.6(1.1) \mathrm{s}$, where the error bars are defined by the error bars of the axial coupling constant $\lambda=-1.2750(9)$ and the CKM matrix element $V_{u d}=0.97427(15)$, agrees well with the experimental values $\tau_{n}^{(\exp )}=878.5(8) \mathrm{s}, \tau_{n}^{(\exp )}=880.7(1.8) \mathrm{s}$ and $\tau_{n}^{(\exp )}=881.6(2.1) \mathrm{s}$, measured by Serebrov et al. [12], Pichlmaier et al. [79] and Arzumanov et al. [80], respectively, and the world average values of the neutron lifetime $\tau_{n}^{\text {(w.a.v.) }}=880.1(1.1) \mathrm{s}, \tau_{n}^{\text {(w.a.v.) }}=880.0(9) \mathrm{s}$ and $\tau_{n}^{\text {(w.a.v.) }}=881.9(1.3) \mathrm{s}$, obtained in $[9$, 81, 82, respectively.

In order to demonstrate the sensitivity of the lifetime of the neutron, calculated in the SM, to the contributions of the radiative and $1 / M$ corrections we propose to rewrite the Fermi integral $f_{n}\left(E_{0}, Z=1\right)$, given by Eq. (41), in the following form

$$
\begin{aligned}
& f_{n}\left(E_{0}, Z=1\right)=\int_{m_{e}}^{E_{0}}\left(E_{0}-E_{e}\right)^{2} \sqrt{E_{e}^{2}-m_{e}^{2}} E_{e} F\left(E_{e}, Z=1\right)\left\{\left(1+k_{1} \frac{\alpha}{\pi} g_{n}\left(E_{e}\right)\right)\right. \\
& \left.+k_{2} \frac{1}{M} \frac{1}{1+3 \lambda^{2}}\left[\left(10 \lambda^{2}-4(\kappa+1) \lambda+2\right) E_{e}-2 \lambda(\lambda-(\kappa+1))\left(E_{0}+\frac{m_{e}^{2}}{E_{e}}\right)\right]\right\} d E_{e}
\end{aligned}
$$

where the coefficients $k_{j}$ for $j=1,2$ are equal to $k_{j}=0$ or $k_{j}=1$ that means that without $k_{j}=0$ and with $k_{j}=1$ corresponding corrections. The numerical values of the lifetime of the neutron for different $k_{j}$ are adduced in Table I. It is seen that the most important contributions come from the radiative corrections.

\begin{tabular}{|c|c|c|}
\hline$\tau_{n}$ & $k_{1}$ & $k_{2}$ \\
\hline $915.3 \mathrm{~s}$ & 0 & 0 \\
\hline $913.7 \mathrm{~s}$ & 0 & 1 \\
\hline $881.0 \mathrm{~s}$ & 1 & 0 \\
\hline $879.6 \mathrm{~s}$ & 1 & 1 \\
\hline
\end{tabular}

TABLE I: The neutron lifetime, calculated for $\lambda=-1.2750$, the radiative corrections $k_{1}=0,1$ and the $1 / M$ corrections $k_{2}=0,1$, caused by the "weak magnetism" and the proton recoil.

For the comparison of the theoretical lifetime of the neutron, defined by Eq. (440), with the expression, which is usually used for the measurement of the CKM matrix element $V_{u d}$ [28] (see also [84]), we transcribe Eq. (40) into the form 28,84

$$
\frac{1}{\tau_{n}}=C_{n}\left|V_{u d}\right|^{2}\left(1+3 \lambda^{2}\right) f(1+\mathrm{RC})
$$

where we have denoted $C_{n}=G_{F}^{2} m_{e}^{5} / 2 \pi^{3}=1.1614 \times 10^{-4} \mathrm{~s}^{-1}$ and $\mathrm{RC}=\left\langle(\alpha / \pi) g_{n}\left(E_{e}\right)\right\rangle=0.03886$, defining the radiative corrections [1, 28] integrated over the phase volume with the account for the proton-electron final-state Coulomb interaction. Then, the phase-space factor $f$, including the $1 / M$ corrections from the "weak magnetism" and the proton recoil, is determined by

$$
\begin{aligned}
& f=\frac{1}{m_{e}^{5}} \int_{m_{e}}^{E_{0}}\left(E_{0}-E_{e}\right)^{2} \sqrt{E_{e}^{2}-m_{e}^{2}} E_{e} F\left(E_{e}, Z=1\right)\left\{1+\frac{1}{M} \frac{1}{1+3 \lambda^{2}}\left[\left(10 \lambda^{2}-4(\kappa+1) \lambda+2\right) E_{e}\right.\right. \\
& \left.\left.-2 \lambda(\lambda-(\kappa+1))\left(E_{0}+\frac{m_{e}^{2}}{E_{e}}\right)\right]\right\} d E_{e}=1.6894
\end{aligned}
$$

The numerical value agrees well with the value $f=1.6887$, calculated in [28] (see also [84]). The factor $1+\mathrm{RC}$ we may represent in the following form $1+\mathrm{RC}=\left(1+\delta_{R}\right)\left(1+\Delta_{R}\right)$, where $\delta_{R}=\left\langle(\alpha / \pi)\left(g_{n}\left(E_{e}\right)-C_{W Z}\right)\right\rangle=0.01505$ is defined by one-photon exchanges and emission only [28, 29] and $\Delta_{R}=(\alpha / \pi) C_{W Z}=0.02381$ is the part of the radiative corrections, induced by $W$-boson and $Z$-boson exchanges and QCD corrections [28, 29] (see also [32]). The phase-space factor $f_{R}$, including the contributions of the radiative corrections, caused by one-photon exchanges and emission only, is equal to $f_{R}=f\left(1+\delta_{R}\right)=1.71483$. It does not contradict the value $f_{R}=1.71385(34)$, used in [84] (see also [85]).

Currently the lifetime of the neutron is proposed to measuring in TU München within the project PENeLOPE, using a superconducting magneto-gravitational trap of ultracold neutrons (UCN) for a precise neutron lifetime measurement [86]. In this experiment the UCN are trapped in a multipole field of a flux density up to $2 \mathrm{~T}$ and bound by a gravitational force at the top. This makes the extraction and detection of the protons possible and allows a direct measurement of neutron decay. A planing accuracy of $0.1 \mathrm{~s}$ and better demands high storage times and good knowledge of systematic errors, which could result from neutron spin flip and high energetic UCN which leave the storage volume only slowly. Therefore, the neutron spectrum is cleaned by an absorber. The big storage volume of $800 \mathrm{dm}^{3}$ and the 
expected high neutron flux of FRMII give more than $10^{7}$ neutrons per filling of the storage volume and meet statistical demands. Of course, the experimental data on the lifetime of the neutron, which should be obtained within this project with a planning accuracy better than $0.1 \mathrm{~s}$, should place new constraints on contributions of interactions beyond the SM.

For the completeness of our analysis we have calculated (see Appendix $\mathrm{H}$ ) the contributions of the proton recoil corrections of order $\alpha / M$, caused by the electron-proton Coulomb interaction in the final state of the neutron $\beta^{--}$ decay. We have shown that these corrections to the lifetime of the neutron and the correlation coefficients are of order $10^{-6}-10^{-5}$. This allows to neglect them for the analysis of contributions of order $10^{-4}$ of interactions beyond the SM.

We would like to note that we have used the experimental value of the axial coupling constant $\lambda=-1.2750(9)$, determined from the experimental data on the electron asymmetry $A_{\exp }\left(E_{e}\right)$ [1, 37]. Such an experimental value of the axial coupling constant has been obtained with an unprecedented accuracy of about $0.07 \%$. The axial coupling constant $\lambda=-1.2750(9)$ agrees well with the axial coupling constants $\lambda=-1.2761_{-17}^{+14}, \lambda=-1.2759_{-44.5}^{+40.9}$ and $\lambda=-1.2756(30)$, obtained recently by the PERKEO (PERKEO II) Collaboration [84] and the UCNA (Ultracold Neutron Asymmetry) Collaboration [87, 88], respectively, the accuracies of which are large compared with the accuracy of the axial coupling constant $\lambda=-1.2750(9)$. The lifetimes of the neutron $\tau_{n}=879(2) \mathrm{s}, \tau_{n}=879(6) \mathrm{s}$ and $\tau_{n}=879(4) \mathrm{s}$, calculated for the axial coupling constants $\lambda=-1.2761_{-17}^{+14}, \lambda=-1.2759_{-44.5}^{+40.9}$ and $\lambda=-1.2756(30)$, respectively, agree with the experimental data $\tau_{n}^{(\exp )}=878.5(8) \mathrm{s}, \tau_{n}^{(\exp )}=880.7(1.8) \mathrm{s}$ and $\tau_{n}^{(\exp )}=881.6(2.1) \mathrm{s}$, measured by Serebrov et al. [12], Pichlmaier et al. [79] and Arzumanov et al. [80], respectively, and the world average values of the neutron lifetime $\tau_{n}^{\text {(w.a.v.) }}=880.1(1.1) \mathrm{s}, \tau_{n}^{\text {(w.a.v.) }}=880.0(9) \mathrm{s}$ and $\tau_{n}^{\text {(w.a.v. })}=881.9(1.3) \mathrm{s}$, obtained in [9, 81, 82, respectively.

The other experimental values of the axial coupling constant $\lambda=-1.266(4), \lambda=-1.2594(38)$ and $\lambda=-1.262(5)$, obtained in [89, 90] and [91], respectively, and cited by [9], lead to the lifetimes of the neutron $\tau_{n}=890(5) \mathrm{s}$, $\tau_{n}=898(5) \mathrm{s}$ and $\tau_{n}=895(7) \mathrm{s}$, which do not agree with the world average values of the neutron lifetime $\tau_{n}^{(\text {w.a.v. })}=$ $880.1(1.1) \mathrm{s}, \tau_{n}^{\text {(w.a.v.) }}=880.0(9) \mathrm{s}$ and $\tau_{n}^{\text {(w.a.v.) }}=881.9(1.3) \mathrm{s}$, obtained in [9, 81, 82], respectively. Moreover, the experimental methods, used in [89, 90] and [91] for the measurements of the electron asymmetry $A_{\exp }\left(E_{e}\right)$, has been recently criticized in [84]. As has been pointed out by Mund et al. [84], in the experiments [89, 90] and [91] large corrections of about $15 \%-30 \%$ should be applied to 1) neutron polarization, 2) magnetic mirror effects, 3) solid angle and 4) background.

For a long time [13]-30] (see also [31, 32]) due to infrared divergences the calculation of the radiative $\beta^{-}-$decay of the neutron has been associated with the calculation of the radiative corrections to the neutron $\beta^{-}-$decay. As has been shown already in [13], the sum of the rates as well as the electron-energy and angular distributions of the continuum-state and radiative $\beta^{-}$-decay modes of the neutron does not suffer from infrared divergences, caused by one-virtual photon exchanges in the continuum-state $\beta^{-}$-decay mode and by the emission of real photons in radiative $\beta^{-}$-decay mode of the neutron.

Nevertheless, the radiative $\beta^{-}$-decay of the neutron $n \rightarrow p+e^{-}+\bar{\nu}_{e}+\gamma$ may be treated as a physical process, which may be observed separately from the neutron $\beta^{-}$-decay $n \rightarrow p+e^{-}+\bar{\nu}_{e}$. For the first time, the theoretical analysis of the radiative $\beta^{-}$-decay of the neutron $n \rightarrow p+e^{-}+\bar{\nu}_{e}+\gamma$ as a physical observable process has been carried out in [49, 50]. First reliable experimental data on the branching ratio of the radiative $\beta^{-}-$decay of the neutron $\mathrm{BR}_{\beta_{c}^{-} \gamma}^{(\exp )}=3.13(35) \times 10^{-3}$, measured for the photon energy region $\omega_{\min }=15 \mathrm{keV} \leq \omega \leq \omega_{\max }=340 \mathrm{keV}$, have been reported by Nico et al. [46]. Then this result has been updated by Cooper et al. 47, 48], who have obtained $\mathrm{BR}_{\beta_{c}^{-} \gamma}^{(\exp )}=3.09(32) \times 10^{-3}$. These experimental values agree well with the theoretical value $\mathrm{BR}_{\beta_{c}^{-} \gamma}=2.85 \times 10^{-3}$, calculated by Gardner within $\mathrm{HB} \chi \mathrm{PT}$ for the same photon energy region [46, 47, 50]. In Appendix B we have carried out the calculation of the rate, the electron-photon energy and photon-energy spectra and angular distributions of the radiative $\beta^{-}$-decay of the neutron with a polarized neutron and unpolarised decay particles. Our results for the branching ratios $\mathrm{BR}_{\beta_{c}^{-} \gamma}=2.87 \times 10^{-3}$ and $\mathrm{BR}_{\beta_{c}^{-} \gamma}=4.45 \times 10^{-3}$, calculated for the photon energy regions $\omega_{\text {min }}=$ $15 \mathrm{keV} \leq \omega \leq 350 \mathrm{keV}$ and $\omega_{\min }=5 \mathrm{keV} \leq \omega \leq E_{0}-m_{e}$, respectively, agree well with the results $\mathrm{BR}_{\beta_{c}^{-} \gamma}=2.85 \times 10^{-3}$ and $\mathrm{BR}_{\beta_{c}^{-} \gamma}=4.41 \times 10^{-3}$, obtained by Gardner [46, 47] and Bernard et al. [50], respectively. Within one standard deviation the branching ratio $\mathrm{BR}_{\beta_{c}^{-} \gamma}=2.87 \times 10^{-3}$ agrees also with the experimental data [46, 47].

The rate of the radiative $\beta^{-}$-decay of the neutron, depending on a photon polarization, has been calculated in [50]. We argue that the more precise theoretical and experimental analysis of the energy spectra and angular distributions of the radiative $\beta^{-}$-decay of the neutron, depending on the polarisations of the neutron and photon, should be of great deal of importance for a test of the SM. We are planning to perform such a theoretical analysis in our forthcoming publication. 


\section{A. Universality of radiative corrections to order $\alpha / \pi$}

The radiative corrections of $\alpha / \pi \sim 10^{-3}$ to the electron-energy spectrum of the neutron $\beta^{-}$-decay, described by the function $g_{n}\left(E_{e}\right)$, are universal for the electron (positron) energy spectra of nuclear and neutron $\beta$-decays 92 , 93. A universality of the radiative corrections to order $\alpha / \pi \sim 10^{-3}$ to neutrino (antineutrino) reactions, induced by weak charged currents, has been pointed out by Kurylov, Ramsey-Musolf and Vogel 94 by example of the neutrino (antineutrino) disintegration of the deuteron with the electron (positron) in the final state. Such a universality has been confirmed in [95] for the cross section for the inverse $\beta$-decay. As has been shown in [95] the radiative corrections, calculated in [94], can be described by the function $f_{A}\left(E_{\bar{\nu}}\right)$ of the antineutrino energy $E_{\bar{\nu}}$, calculated by Vogel [96], Fayans [97], Fukugita and Kubota [98], and Raha, Myhrer and Kudobera [99] (see also [95]) and caused by one-virtual photon exchanges and the radiative inverse $\beta$-decay, and the constant part, caused by the electroweak boson exchanges. In turn, as has been shown by Sirlin [100], the radiative corrections, caused by one-virtual photon exchanges and the bremsstrahlung, to neutrino (antineutrino) energy spectra of the $\beta$-decays are also described by the function $f_{A}\left(E_{\bar{\nu}}\right)$ (see also 95]). A nice review of the radiative corrections in precision electroweak physics has been recently written by Sirlin and Ferroglia [101].

\section{ACKNOWLEDGEMENTS}

We are very grateful to H. Abele and A. P. Serebrov for numerous discussions of the results, obtained in this paper, and advices on the content and structure of the paper. We thank G. Konrad for discussions of the measurements of the correlation coefficient $a_{0}$ by means of the measurements of the electron-proton energy distribution and the proton-energy spectrum of the neutron $\beta^{-}$-decay. We acknowledge fruitful discussions with M. Ramsey-Musolf and M. González-Alonso. We are grateful to W. Marciano for the discussions of the radiative corrections to the $\beta^{-}-$decay of the neutron. We thank S. Gardner and V. Gudkov for the discussions of their results on the analysis of the $\beta^{-}-$ decay modes of the neutron, which were put in the ground of our paper as well as the results, obtained by Sirlin et al..

The theoretical analysis of the sensitivity of 1) the electron-proton energy distribution and the proton-energy spectrum, 2) the electron, antineutrino and proton asymmetries of correlations between the neutron spin and the 3-momenta of the decay particles and 3) the lifetime of the neutron is carried out according to the experimental program on the contract I534-N20 PERC, the theoretical program on the contract I689-N16, supported both by the Austrian "Fonds zur Förderung der Wissenschaftlichen Forschung" (FWF), and the experimental program of the experimental group of the Petersburg Nuclear Physics Institute (PNPI), headed by A. P. Serebrov, on the contract No. 11-02-91000 -ANF_a, supported by the Russian Foundation for Basic Research.

This work was supported by the Austrian "Fonds zur Förderung der Wissenschaftlichen Forschung" (FWF) under the contracts I689-N16, I534-N20 PERC and I862-N20 and by the Russian Foundation for Basic Research under the contract No. 11-02-91000-ANF_a and in part by the U.S. Department of Energy contract No. DE-FG02-08ER41531, No. DE-AC02-06CH11357 and the Wisconsin Alumni Research Foundation.

Appendix A: Amplitude of continuum-state $\beta^{-}$-decay of neutron with "weak magnetism" and proton recoil corrections to order $1 / M$

The amplitude of the continuum-state $\beta^{-}$-decay of the neutron we rewrite as follows

$$
M\left(n \rightarrow p e^{-} \bar{\nu}_{e}\right)=-\frac{G_{F}}{\sqrt{2}} V_{u d} \mathcal{M}_{\beta_{c}^{-}}
$$

where $\mathcal{M}_{\beta_{c}^{-}}=\left[\bar{u}_{p} O_{\mu} u_{n}\right]\left[\bar{u}_{e} \gamma^{\mu}\left(1-\gamma^{5}\right) v_{\bar{\nu}}\right]$ and the matrix $O_{\mu}$ takes the form

$$
O_{\mu}=\gamma_{\mu}\left(1+\lambda \gamma^{5}\right)+i \frac{\kappa}{2 M} \sigma_{\mu \nu}\left(k_{p}-k_{n}\right)^{\nu}
$$

In terms of the time and space components of the matrix $O_{\mu}=\left(O^{0},-\vec{O}\right)$ the amplitude $\mathcal{M}_{\beta_{c}^{-}}$is defined by

$$
\mathcal{M}_{\beta_{c}^{-}}=\left[\bar{u}_{p} O^{0} u_{n}\right]\left[\bar{u}_{e} \gamma^{0}\left(1-\gamma^{5}\right) v_{\bar{\nu}}\right]-\left[\bar{u}_{p} \vec{O} u_{n}\right] \cdot\left[\bar{u}_{e} \vec{\gamma}\left(1-\gamma^{5}\right) v_{\bar{\nu}}\right]
$$


The time $O^{0}$ and spacial $\vec{O}$ components of the matrix $O_{\mu}$ we determine to first in the large $M$ expansion. They read

$$
O^{0}=\left(\begin{array}{cc}
1 & \lambda+\frac{\kappa}{2 M}\left(\vec{\sigma} \cdot \vec{k}_{p}\right) \\
-\lambda+\frac{\kappa}{2 M}\left(\vec{\sigma} \cdot \vec{k}_{p}\right) & -1
\end{array}\right)
$$

and

$$
\vec{O}=\left(\begin{array}{cc}
\lambda \vec{\sigma}+i \frac{\kappa}{2 M}\left(\vec{\sigma} \times \vec{k}_{p}\right) & \vec{\sigma}\left(1-\frac{\kappa}{2 M} E_{0}\right) \\
-\vec{\sigma}\left(1+\frac{\kappa}{2 M} E_{0}\right) & -\lambda \vec{\sigma}+i \frac{\kappa}{2 M}\left(\vec{\sigma} \times \vec{k}_{p}\right)
\end{array}\right)
$$

where we have kept the terms of order $1 / M$ only. For the calculation of the amplitude of the $\beta^{-}-$decay of the neutron we use the Dirac bispinorial wave functions of the neutron and the proton

$$
u_{n}\left(\overrightarrow{0}, \sigma_{n}\right)=\sqrt{2 m_{n}}\left(\begin{array}{c}
\varphi_{n} \\
0
\end{array}\right) \quad, \quad u_{p}\left(\vec{k}_{p}, \sigma_{p}\right)=\sqrt{E_{p}+m_{p}}\left(\begin{array}{c}
\varphi_{p} \\
\frac{\vec{\sigma} \cdot \vec{k}_{p}}{E_{p}+m_{p}} \varphi_{p}
\end{array}\right)
$$

where the Pauli spinorial wave functions $\varphi_{n}$ and $\varphi_{p}$ depend on the polarisations $\sigma_{n}$ and $\sigma_{p}$, respectively. The matrix elements $\left[\bar{u}_{p} O^{0} u_{n}\right]$ and $\left[\bar{u}_{p} \vec{O} u_{n}\right]$ are equal to

$$
\left[\bar{u}_{p} O^{0} u_{n}\right]=\sqrt{2 m_{n}\left(E_{p}+m_{p}\right)}\left\{\left[\varphi_{p}^{\dagger} \varphi_{n}\right]+\frac{\lambda}{2 M}\left[\varphi_{p}^{\dagger}\left(\vec{\sigma} \cdot \vec{k}_{p}\right) \varphi_{n}\right]\right\}
$$

and

$$
\left[\bar{u}_{p} \vec{O} u_{n}\right]=\sqrt{2 m_{n}\left(E_{p}+m_{p}\right)}\left\{\lambda\left[\varphi_{p}^{\dagger} \vec{\sigma} \varphi_{n}\right]+i \frac{\kappa}{2 M}\left[\varphi_{p}^{\dagger}\left(\vec{\sigma} \times \vec{k}_{p}\right) \varphi_{n}\right]+\frac{1}{2 M}\left[\varphi_{p}^{\dagger}\left(\vec{\sigma} \cdot \vec{k}_{p}\right) \vec{\sigma} \varphi_{n}\right]\right\}
$$

where in curly brackets we have kept the contributions of the terms of order $1 / M$ only. Using the relation $\left(\vec{\sigma} \cdot \vec{k}_{p}\right) \vec{\sigma}=$ $\vec{k}_{p}+i\left(\vec{\sigma} \times \vec{k}_{p}\right)$ we rewrite the r.h.s. of Eq. (A-8) as follows

$$
\left[\bar{u}_{p} \vec{O} u_{n}\right]=\sqrt{2 m_{n}\left(E_{p}+m_{p}\right)}\left\{\lambda\left[\varphi_{p}^{\dagger} \vec{\sigma} \varphi_{n}\right]+i \frac{\kappa+1}{2 M}\left[\varphi_{p}^{\dagger}\left(\vec{\sigma} \times \vec{k}_{p}\right) \varphi_{n}\right]+\frac{\vec{k}_{p}}{2 M}\left[\varphi_{p}^{\dagger} \varphi_{n}\right]\right\} .
$$

Thus, the amplitude $\mathcal{M}_{\beta_{c}^{-}}$is given by

$$
\begin{aligned}
& \mathcal{M}_{\beta_{c}^{-}}=\sqrt{2 m_{n}\left(E_{p}+m_{p}\right)}\left\{\left[\varphi_{p}^{\dagger} \varphi_{n}\right]\left[\bar{u}_{e} \gamma^{0}\left(1-\gamma^{5}\right) v_{\bar{\nu}}\right]-\lambda\left[\varphi_{p}^{\dagger} \vec{\sigma} \varphi_{n}\right] \cdot\left[\bar{u}_{e} \vec{\gamma}\left(1-\gamma^{5}\right) v_{\bar{\nu}}\right]+\frac{\lambda}{2 M}\left[\varphi_{p}^{\dagger}\left(\vec{\sigma} \cdot \vec{k}_{p}\right) \varphi_{n}\right]\right. \\
& \left.\times\left[\bar{u}_{e} \gamma^{0}\left(1-\gamma^{5}\right) v_{\bar{\nu}}\right]-i \frac{\kappa+1}{2 M}\left[\varphi_{p}^{\dagger}\left(\vec{\sigma} \times \vec{k}_{p}\right) \varphi_{n}\right] \cdot\left[\bar{u}_{e} \vec{\gamma}\left(1-\gamma^{5}\right) v_{\bar{\nu}}\right]-\frac{\vec{k}_{p}}{2 M}\left[\varphi_{p}^{\dagger} \varphi_{n}\right] \cdot\left[\bar{u}_{e} \vec{\gamma}\left(1-\gamma^{5}\right) v_{\bar{\nu}}\right]\right\} .
\end{aligned}
$$

For the transformation of the last term we use the following identity

$$
-\frac{\vec{k}_{p}}{2 M}\left[\varphi_{p}^{\dagger} \varphi_{n}\right] \cdot\left[\bar{u}_{e} \vec{\gamma}\left(1-\gamma^{5}\right) v_{\bar{\nu}}\right]=\frac{E_{0}}{2 M}\left[\varphi_{p}^{\dagger} \varphi_{n}\right]\left[\bar{u}_{e} \gamma^{0}\left(1-\gamma^{5}\right) v_{\bar{\nu}}\right]-\frac{m_{e}}{2 M}\left[\varphi_{p}^{\dagger} \varphi_{n}\right]\left[\bar{u}_{e}\left(1-\gamma^{5}\right) v_{\bar{\nu}}\right]
$$

based on the Dirac equation for the electron and antineutrino. Substituting Eq.A-11 into Eq.A-10 we obtain

$$
\begin{aligned}
& \mathcal{M}_{\beta_{c}^{-}}=\sqrt{2 m_{n}\left(E_{p}+m_{p}\right)}\left\{\left(1+\frac{E_{0}}{2 M}\right)\left[\varphi_{p}^{\dagger} \varphi_{n}\right]\left[\bar{u}_{e} \gamma^{0}\left(1-\gamma^{5}\right) v_{\bar{\nu}}\right]-\lambda\left[\varphi_{p}^{\dagger} \vec{\sigma} \varphi_{n}\right] \cdot\left[\bar{u}_{e} \vec{\gamma}\left(1-\gamma^{5}\right) v_{\bar{\nu}}\right]+\frac{\lambda}{2 M}\left[\varphi_{p}^{\dagger}\left(\vec{\sigma} \cdot \vec{k}_{p}\right) \varphi_{n}\right]\right. \\
& \left.\times\left[\bar{u}_{e} \gamma^{0}\left(1-\gamma^{5}\right) v_{\bar{\nu}}\right]-i \frac{\kappa+1}{2 M}\left[\varphi_{p}^{\dagger}\left(\vec{\sigma} \times \vec{k}_{p}\right) \varphi_{n}\right] \cdot\left[\bar{u}_{e} \vec{\gamma}\left(1-\gamma^{5}\right) v_{\bar{\nu}}\right]-\frac{m_{e}}{2 M}\left[\varphi_{p}^{\dagger} \varphi_{n}\right]\left[\bar{u}_{e}\left(1-\gamma^{5}\right) v_{\bar{\nu}}\right]\right\} .
\end{aligned}
$$

The next step of the calculation is to expand the normalization factor $\sqrt{E_{p}+m_{p}}$ of the bispinorial wave function of the proton. This gives

$$
\sqrt{2 m_{n}\left(E_{p}+m_{p}\right)}=2 m_{n}\left(1-\frac{E_{0}}{2 M}\right),
$$


where we have kept the next-to-leading terms in the large $M$ expansion.

Thus the amplitude $\mathcal{M}_{\beta_{c}^{-}}$, calculated to next-to-leading order order in the large $M$ expansion, is

$$
\begin{aligned}
& \mathcal{M}_{\beta_{c}^{-}}=2 m_{n}\left\{\left[\varphi_{p}^{\dagger} \varphi_{n}\right]\left[\bar{u}_{e} \gamma^{0}\left(1-\gamma^{5}\right) v_{\bar{\nu}}\right]-\tilde{\lambda}\left[\varphi_{p}^{\dagger} \vec{\sigma} \varphi_{n}\right] \cdot\left[\bar{u}_{e} \vec{\gamma}\left(1-\gamma^{5}\right) v_{\bar{\nu}}\right]+\frac{\tilde{\lambda}}{2 M}\left[\varphi_{p}^{\dagger}\left(\vec{\sigma} \cdot \vec{k}_{p}\right) \varphi_{n}\right]\right. \\
& \left.\times\left[\bar{u}_{e} \gamma^{0}\left(1-\gamma^{5}\right) v_{\bar{\nu}}\right]-i \frac{\kappa+1}{2 M}\left[\varphi_{p}^{\dagger}\left(\vec{\sigma} \times \vec{k}_{p}\right) \varphi_{n}\right] \cdot\left[\bar{u}_{e} \vec{\gamma}\left(1-\gamma^{5}\right) v_{\bar{\nu}}\right]-\frac{m_{e}}{2 M}\left[\varphi_{p}^{\dagger} \varphi_{n}\right]\left[\bar{u}_{e}\left(1-\gamma^{5}\right) v_{\bar{\nu}}\right]\right\},
\end{aligned}
$$

where we have denoted $\tilde{\lambda}=\lambda\left(1-E_{0} / 2 M\right)$. The hermitian conjugate amplitude takes the form

$$
\begin{aligned}
& \mathcal{M}_{\beta_{c}^{-}}^{\dagger}=2 m_{n}\left\{\left[\varphi_{n}^{\dagger} \varphi_{p}\right]\left[\bar{v}_{\bar{\nu}} \gamma^{0}\left(1-\gamma^{5}\right) u_{e}\right]-\tilde{\lambda}^{*}\left[\varphi_{n}^{\dagger} \vec{\sigma} \varphi_{p}\right] \cdot\left[\bar{v}_{\bar{\nu}} \vec{\gamma}\left(1-\gamma^{5}\right) u_{e}\right]+\frac{\tilde{\lambda}^{*}}{2 M}\left[\varphi_{n}^{\dagger}\left(\vec{\sigma} \cdot \vec{k}_{p}\right) \varphi_{p}\right]\right. \\
& \left.\times\left[\bar{v}_{\bar{\nu}} \gamma^{0}\left(1-\gamma^{5}\right) u_{e}\right]+i \frac{\kappa+1}{2 M}\left[\varphi_{n}^{\dagger}\left(\vec{\sigma} \times \vec{k}_{p}\right) \varphi_{p}\right] \cdot\left[\bar{v}_{\bar{\nu}} \vec{\gamma}\left(1-\gamma^{5}\right) u_{e}\right]-\frac{m_{e}}{2 M}\left[\varphi_{n}^{\dagger} \varphi_{p}\right]\left[\bar{v}_{\bar{\nu}}\left(1+\gamma^{5}\right) u_{e}\right]\right\} .
\end{aligned}
$$

Substituting Eq.A-14 into Eq.A-1 we arrive at the amplitude of the continuum-state $\beta^{-}$-decay of the neutron, taking into account the contributions of the "weak magnetism" and the proton recoil, calculated to next-to-leading order in the $1 / M$ expansion.

The electron-energy and angular distribution is proportional to $\frac{1}{2} \sum_{\text {pol }}\left|\mathcal{M}_{\beta_{c}^{-}}\right|^{2}$, where we sum over all polarisations of the interacting particles. Recall that the antineutrino is polarized in the direction parallel to its $3-$ momentum. The quantity $\frac{1}{2} \sum_{\text {pol }}\left|\mathcal{M}_{\beta_{c}^{-}}\right|^{2}$ is equal to

$$
\begin{aligned}
& \sum_{\text {pol }} \frac{\left|\mathcal{M}_{\beta_{c}^{-}}\right|^{2}}{4 m_{n}^{2}}=\operatorname{tr}\left\{1+\vec{\xi}_{n} \cdot \vec{\sigma}\right\} \operatorname{tr}\left\{\left(\hat{k}_{e}+m_{e}\right) \gamma^{0} \hat{k} \gamma^{0}\left(1-\gamma^{5}\right)\right\}-\tilde{\lambda} \operatorname{tr}\left\{\left(1+\vec{\xi}_{n} \cdot \vec{\sigma}\right) \vec{\sigma}\right\} \cdot \operatorname{tr}\left\{\left(\hat{k}_{e}+m_{e}\right) \vec{\gamma} \hat{k}^{0}\left(1-\gamma^{5}\right)\right\} \\
& -\tilde{\lambda}^{*} \operatorname{tr}\left\{\left(1+\vec{\xi}_{n} \cdot \vec{\sigma}\right) \vec{\sigma}\right\} \cdot \operatorname{tr}\left\{\left(\hat{k}_{e}+m_{e}\right) \gamma^{0} \hat{k} \vec{\gamma}\left(1-\gamma^{5}\right)\right\}+|\tilde{\lambda}|^{2} \operatorname{tr}\left\{\left(1+\vec{\xi}_{n} \cdot \vec{\sigma}\right) \sigma^{i} \sigma^{j}\right\} \operatorname{tr}\left\{\left(\hat{k}_{e}+m_{e}\right) \gamma^{j} \hat{k} \gamma^{i}\left(1-\gamma^{5}\right)\right\} \\
& -\frac{m_{e}}{2 M} \operatorname{tr}\left\{\left(1+\vec{\xi}_{n} \cdot \vec{\sigma}\right)\right\} \operatorname{tr}\left\{\left(\hat{k}_{e}+m_{e}\right) \gamma^{0} \hat{k}\left(1+\gamma^{5}\right)\right\}-\frac{m_{e}}{2 M} \operatorname{tr}\left\{\left(1+\vec{\xi}_{n} \cdot \vec{\sigma}\right)\right\} \operatorname{tr}\left\{\left(\hat{k}_{e}+m_{e}\right) \hat{k} \gamma^{0}\left(1-\gamma^{5}\right)\right\} \\
& +\tilde{\lambda} \frac{m_{e}}{2 M} \operatorname{tr}\left\{\left(1+\vec{\xi}_{n} \cdot \vec{\sigma}\right) \vec{\sigma}\right\} \cdot \operatorname{tr}\left\{\left(\hat{k}_{e}+m_{e}\right) \vec{\gamma} \hat{k}\left(1+\gamma^{5}\right)\right\}+\tilde{\lambda}^{*} \frac{m_{e}}{2 M} \operatorname{tr}\left\{\left(1+\vec{\xi}_{n} \cdot \vec{\sigma}\right) \vec{\sigma}\right\} \cdot \operatorname{tr}\left\{\left(\hat{k}_{e}+m_{e}\right) \hat{k} \vec{\gamma}\left(1-\gamma^{5}\right)\right\} \\
& +\frac{\tilde{\lambda}}{2 M} \operatorname{tr}\left\{\left(1+\vec{\xi}_{n} \cdot \vec{\sigma}\right)\left(\vec{\sigma} \cdot \vec{k}_{p}\right)\right\} \operatorname{tr}\left\{\left(\hat{k}_{e}+m_{e}\right) \gamma^{0} \hat{k} \gamma^{0}\left(1-\gamma^{5}\right)\right\} \\
& +\frac{\tilde{\lambda}^{*}}{2 M} \operatorname{tr}\left\{\left(1+\vec{\xi}_{n} \cdot \vec{\sigma}\right)\left(\vec{\sigma} \cdot \vec{k}_{p}\right)\right\} \operatorname{tr}\left\{\left(\hat{k}_{e}+m_{e}\right) \gamma^{0} \hat{k} \gamma^{0}\left(1-\gamma^{5}\right)\right\} \\
& -\frac{|\tilde{\lambda}|^{2}}{2 M} \operatorname{tr}\left\{\left(1+\vec{\xi}_{n} \cdot \vec{\sigma}\right)\left(\vec{\sigma} \cdot \vec{k}_{p}\right) \vec{\sigma}\right\} \cdot \operatorname{tr}\left\{\left(\hat{k}_{e}+m_{e}\right) \vec{\gamma} \hat{k} \gamma^{0}\left(1-\gamma^{5}\right)\right\} \\
& -\frac{|\tilde{\lambda}|^{2}}{2 M} \operatorname{tr}\left\{\left(1+\vec{\xi}_{n} \cdot \vec{\sigma}\right) \vec{\sigma}\left(\vec{\sigma} \cdot \vec{k}_{p}\right)\right\} \cdot \operatorname{tr}\left\{\left(\hat{k}_{e}+m_{e}\right) \gamma^{0} \hat{k} \vec{\gamma}\left(1-\gamma^{5}\right)\right\} \\
& -i \frac{\kappa+1}{2 M} \operatorname{tr}\left\{\left(1+\vec{\xi}_{n} \cdot \vec{\sigma}\right)\left(\vec{\sigma} \times \vec{k}_{p}\right)\right\} \cdot \operatorname{tr}\left\{\left(\hat{k}_{e}+m_{e}\right) \vec{\gamma} \hat{k} \gamma^{0}\left(1-\gamma^{5}\right)\right\} \\
& +i \frac{\kappa+1}{2 M} \operatorname{tr}\left\{\left(1+\vec{\xi}_{n} \cdot \vec{\sigma}\right)\left(\vec{\sigma} \times \vec{k}_{p}\right)\right\} \cdot \operatorname{tr}\left\{\left(\hat{k}_{e}+m_{e}\right) \gamma^{0} \hat{k} \vec{\gamma}\left(1-\gamma^{5}\right)\right\} \\
& +i \tilde{\lambda}^{*} \frac{\kappa+1}{2 M} \operatorname{tr}\left\{(1+\vec{\xi} \vec{n} \cdot \vec{\sigma}) \sigma^{j}\left(\vec{\sigma} \times \vec{k}_{p}\right)^{\ell}\right\} \operatorname{tr}\left\{\left(\hat{k}_{e}+m_{e}\right) \gamma^{\ell} \hat{k} \gamma^{j}\left(1-\gamma^{5}\right)\right\} \\
& -i \tilde{\lambda} \frac{\kappa+1}{2 M} \operatorname{tr}\left\{\left(1+\vec{\xi}_{n} \cdot \vec{\sigma}\right)\left(\vec{\sigma} \times \vec{k}_{p}\right)^{\ell} \sigma^{j}\right\} \operatorname{tr}\left\{\left(\hat{k}_{e}+m_{e}\right) \gamma^{j} \hat{k} \gamma^{\ell}\left(1-\gamma^{5}\right)\right\} .
\end{aligned}
$$

Since in our analysis the axial coupling constant $\lambda$ is real, below we set $\lambda^{*}=\lambda$. Calculating the traces with the real axial coupling constant we obtain the following result

$$
\begin{aligned}
& \sum_{\text {pol }} \frac{\left|\mathcal{M}_{\beta_{c}^{-}}\right|^{2}}{32 m_{n}^{2}}=\left(1+3 \tilde{\lambda}^{2}\right) E_{e} E+\left(1-\tilde{\lambda}^{2}\right)\left(\vec{k}_{e} \cdot \vec{k}\right)-2 \tilde{\lambda}(1+\tilde{\lambda})\left(\vec{\xi}_{n} \cdot \vec{k}_{e}\right) E-2 \tilde{\lambda}(1-\tilde{\lambda})\left(\vec{\xi}_{n} \cdot \vec{k}\right) E_{e} \\
& +\frac{1}{M}\left\{-m_{e}^{2} E+\tilde{\lambda} m_{e}^{2}\left(\vec{\xi}_{n} \cdot \vec{k}\right)-\tilde{\lambda}\left(\vec{\xi}_{n} \cdot \vec{k}_{e}+\vec{\xi}_{n} \cdot \vec{k}\right)\left(E_{e} E+\vec{k}_{e} \cdot \vec{k}\right)+\tilde{\lambda}^{2}\left[\left(E_{e}-\vec{\xi}_{n} \cdot \vec{k}_{e}\right)\left(\vec{k}_{e} \cdot \vec{k}+E^{2}\right)\right.\right. \\
& \left.+\left(E+\vec{\xi}_{n} \cdot \vec{k}\right)\left(k_{e}^{2}+\vec{k}_{e} \cdot \vec{k}\right)\right]-(\kappa+1)\left[\left(\vec{\xi}_{n} \cdot \vec{k}_{e}\right)\left(\vec{k}_{e} \cdot \vec{k}+E^{2}\right)-\left(\vec{\xi}_{n} \cdot \vec{k}\right)\left(k_{e}^{2}+\vec{k}_{e} \cdot \vec{k}\right)\right] \\
& -2(\kappa+1) \tilde{\lambda}\left[E\left(k_{e}^{2}+\vec{k}_{e} \cdot \vec{k}\right)-E_{e}\left(\vec{k}_{e} \cdot \vec{k}+E^{2}\right)\right]+2(\kappa+1) \tilde{\lambda}\left(\vec{\xi}_{n} \cdot \vec{k}_{e}+\vec{\xi}_{n} \cdot \vec{k}\right) E_{e} E
\end{aligned}
$$




$$
\left.-(\kappa+1) \tilde{\lambda}\left[\left(\vec{\xi}_{n} \cdot \vec{k}_{e}\right)\left(\vec{k}_{e} \cdot \vec{k}+E^{2}\right)+\left(\vec{\xi}_{n} \cdot \vec{k}\right)\left(k_{e}^{2}+\vec{k}_{e} \cdot \vec{k}\right)\right]\right\} .
$$

Taking into account the contribution of the phase volume Eq.(4) (see also Eq.A-23) and keeping only the terms of order $1 / M$ we have

$$
\begin{aligned}
& \Phi_{\beta_{c}^{-}}\left(\vec{k}_{e}, \vec{k}\right) \sum_{\mathrm{pol}} \frac{\left|\mathcal{M}_{\beta_{c}^{-}}\right|^{2}}{32 m_{n}^{2}}=\left(1+3 \lambda^{2}\right) E_{e} E+\left(1-\lambda^{2}\right)\left(\vec{k}_{e} \cdot \vec{k}\right)-2 \lambda(1+\lambda)\left(\vec{\xi}_{n} \cdot \vec{k}_{e}\right) E-2 \lambda(1-\lambda)\left(\vec{\xi}_{n} \cdot \vec{k}\right) E_{e} \\
& +\frac{1}{M}\left\{\left[-3 \lambda^{2} E_{e} E+\lambda^{2}\left(\vec{k}_{e} \cdot \vec{k}\right)+\lambda(2 \lambda+1)\left(\vec{\xi}_{n} \cdot \vec{k}_{e}\right) E-\lambda(2 \lambda-1)\left(\vec{\xi}_{n} \cdot \vec{k}\right) E_{e}\right] E_{0}+\left[3\left(1+3 \lambda^{2}\right) E_{e} E\right.\right. \\
& \left.+3\left(1-\lambda^{2}\right)\left(\vec{k}_{e} \cdot \vec{k}\right)-6 \lambda(1+\lambda)\left(\vec{\xi}_{n} \cdot \vec{k}_{e}\right) E-6 \lambda(1-\lambda)\left(\vec{\xi}_{n} \cdot \vec{k}\right) E_{e}\right] E_{e}+\left[-3\left(1+3 \lambda^{2}\right)\left(\vec{k}_{e} \cdot \vec{k}\right) E_{e}-3\left(1-\lambda^{2}\right)\right. \\
& \left.\times \frac{\left(\vec{k}_{e} \cdot \vec{k}\right)^{2}}{E}+6 \lambda(1+\lambda)\left(\vec{\xi}_{n} \cdot \vec{k}_{e}\right)\left(\vec{k}_{e} \cdot \vec{k}\right)+6 \lambda(1-\lambda)\left(\vec{\xi}_{n} \cdot \vec{k}\right)\left(\vec{k}_{e} \cdot \vec{k}\right) \frac{E_{e}}{E}\right]-m_{e}^{2} E+\lambda m_{e}^{2}\left(\vec{\xi}_{n} \cdot \vec{k}\right) \\
& -\lambda\left(\vec{\xi}_{n} \cdot \vec{k}_{e}+\vec{\xi}_{n} \cdot \vec{k}\right)\left(E_{e} E+\vec{k}_{e} \cdot \vec{k}\right)+\lambda^{2}\left[\left(E_{e}-\vec{\xi}_{n} \cdot \vec{k}_{e}\right)\left(\vec{k}_{e} \cdot \vec{k}+E^{2}\right)+\left(E+\vec{\xi}_{n} \cdot \vec{k}\right)\left(k_{e}^{2}+\vec{k}_{e} \cdot \vec{k}\right)\right] \\
& -(\kappa+1)\left[\left(\vec{\xi}_{n} \cdot \vec{k}_{e}\right)\left(\vec{k}_{e} \cdot \vec{k}+E^{2}\right)-\left(\vec{\xi}_{n} \cdot \vec{k}\right)\left(k_{e}^{2}+\vec{k}_{e} \cdot \vec{k}\right)\right]-2(\kappa+1) \lambda\left[E\left(k_{e}^{2}+\vec{k}_{e} \cdot \vec{k}\right)-E_{e}\left(\vec{k}_{e} \cdot \vec{k}+E^{2}\right)\right] \\
& \left.+2(\kappa+1) \lambda\left(\vec{\xi}_{n} \cdot \vec{k}_{e}+\vec{\xi}_{n} \cdot \vec{k}\right) E_{e} E-(\kappa+1) \lambda\left[\left(\vec{\xi}_{n} \cdot \vec{k}_{e}\right)\left(\vec{k}_{e} \cdot \vec{k}+E^{2}\right)+\left(\vec{\xi}_{n} \cdot \vec{k}\right)\left(k_{e}^{2}+\vec{k}_{e} \cdot \vec{k}\right)\right]\right\} \cdot
\end{aligned}
$$

The function $\Phi_{\beta_{c}^{-}}\left(\vec{k}_{e}, \vec{k}\right)$ is defined by the integral over the antineutrino energy $E$

$$
\Phi_{\beta_{c}^{-}}\left(\vec{k}_{e}, \vec{k}\right)=\int_{0}^{\infty} \delta(f(E)) \frac{m_{n}}{E_{p}} \frac{E^{2} d E}{\left(E_{0}-E_{e}\right)^{2}},
$$

where the function $f(E)$ is $f(E)=m_{n}-E_{p}-E_{e}-E$ and $E_{p}=\sqrt{m_{p}^{2}+\left(\vec{k}_{e}+\vec{k}\right)^{2}}$ is the proton energy after the integration over the $3-$ momentum of the proton, giving $\vec{k}_{p}=-\vec{k}_{e}-\vec{k}$. Using the properties of the $\delta$-function the result of the integration over $E$ is equal to

$$
\Phi_{\beta_{c}^{-}}\left(\vec{k}_{e}, \vec{k}\right)=\left.\frac{m_{n}}{E_{p}} \frac{E^{2}}{\left(E_{0}-E_{e}\right)^{2}} \frac{1}{\left|\frac{d f(E)}{d E}\right|}\right|_{E=E_{r}},
$$

where $E_{r}$ is the root of the equation $f\left(E_{r}\right)=0$. To next-to-leading order in the large $M$ expansion the root $E_{r}$ is equal to

$$
E_{r}=\left(E_{0}-E_{e}\right)\left(1+\frac{1}{M}\left(E_{e}-k_{e} \cos \vartheta_{e \bar{\nu}}\right)\right)
$$

where $\cos \vartheta_{e \bar{\nu}}=\vec{k}_{e} \cdot \vec{k} / k_{e} E$. Since

$$
\left.\frac{m_{n}}{E_{p}} \frac{1}{\left|\frac{d f(E)}{d E}\right|}\right|_{E=E_{r}}=\frac{m_{n}}{m_{n}-E_{e}+k_{e} \cos \vartheta_{e \bar{\nu}}}=1+\frac{1}{M}\left(E_{e}-k_{e} \cos \vartheta_{e \bar{\nu}}\right),
$$

where we have kept the terms of order $1 / M$ only, using Eq.A-21 and Eq. (A-22) for the function $\Phi_{\beta_{c}^{-}}\left(\vec{k}_{e}, \vec{k}\right)$ we obtain the following expression

$$
\Phi_{\beta_{c}^{-}}\left(\vec{k}_{e}, \vec{k}\right)=1+\frac{3}{M}\left(E_{e}-k_{e} \cos \vartheta_{e \bar{\nu}}\right)=1+\frac{3}{M}\left(E_{e}-\frac{\vec{k}_{e} \cdot \vec{k}}{E}\right) .
$$

We have used this expression for the calculation of Eq.(A-18), which defines the correlation coefficients of the continuum-state $\beta^{-}$-decay of the neutron, calculated to next-to-leading order in the large $M$ expansion (see Eq.(6) ).

\section{Appendix B: Radiative $\beta^{-}$-decay of neutron}

In this Appendix we calculate the amplitude, the rate, the photon-electron and photon energy and angular distributions of the radiative $\beta^{-}$-decay $n \rightarrow p+e^{-}+\bar{\nu}_{e}+\gamma$ of the neutron. The Hamilton operator of weak interactions 
is defined by Eq.(11), whereas the Hamilton operator of electromagnetic interactions is

$$
\mathcal{H}_{\mathrm{em}}(x)=e\left[\bar{\psi}_{p}(x) \gamma^{\mu} \psi_{p}(x)-\bar{\psi}_{e}(x) \gamma^{\mu} \psi_{e}(x)\right] A_{\mu}(x),
$$

where $e$ is the electric charge of the proton and $A_{\mu}(x)=(0,-\vec{A}(x))$ is the electromagnetic vector potential, taken in the Coulomb gauge $\operatorname{div} \vec{A}(x)=035$.

For the calculation of the amplitude of the radiative $\beta^{-}$-decay $n \rightarrow p+e^{-}+\bar{\nu}_{e}+\gamma$ we take into account the contributions of the intermediate proton and electron states and drop the "weak magnetism" and proton recoil corrections. This gives

$$
\begin{aligned}
M\left(n \rightarrow p e^{-} \bar{\nu}_{e} \gamma\right) & =e \frac{G_{F}}{\sqrt{2}} V_{u d}\left[\bar{u}_{p} \gamma^{\mu}\left(1+\lambda \gamma^{5}\right) u_{n}\right] \frac{1}{2 q \cdot k_{e}}\left[\bar{u}_{e}\left(2 \varepsilon^{*} \cdot k_{e}+\hat{\varepsilon}^{*} \hat{q}\right) \gamma_{\mu}\left(1-\gamma^{5}\right) v_{\bar{\nu}}\right] \\
& -e \frac{G_{F}}{\sqrt{2}} V_{u d} \frac{1}{2 q \cdot k_{p}}\left[\bar{u}_{p}\left(2 \varepsilon^{*} \cdot k_{p}+\hat{\varepsilon}^{*} \hat{q}\right) \gamma^{\mu}\left(1+\lambda \gamma^{5}\right) u_{n}\right]\left[\bar{u}_{e} \gamma_{\mu}\left(1-\gamma^{5}\right) v_{\bar{\nu}}\right]
\end{aligned}
$$

where $\varepsilon=(0, \vec{\varepsilon})$ and $q=(\omega, \vec{q})$ are the polarization vector and 4-momentum of the photon, obeying the constraint $\varepsilon \cdot q=0$.

We calculate the amplitude of the radiative $\beta^{-}$-decay of the neutron to leading order in the large proton mass (or large $M)$ expansion. This agrees well with 1) the neglect of the contributions of order $(\alpha / \pi)\left(E_{0} / M\right) \sim 10^{-6}($ see the discussion below Eq.(20) ) and 2) the assertion [47, 48], that the electron-photon energy spectrum, the photon polarization observables and the rate of the radiative $\beta^{-}$-decay of the neutron are dominated by the electron emission of photons. In such an approximation the amplitude of the radiative $\beta^{-}-$decay takes the form

$$
M\left(n \rightarrow p e^{-} \bar{\nu}_{e} \gamma\right)=e \frac{G_{F}}{\sqrt{2}} V_{u d} \frac{m_{n}}{\omega} \frac{\mathcal{M}_{\beta_{c}^{-} \gamma}}{E_{e}-\vec{n} \cdot \vec{k}_{e}}
$$

where $\vec{n}=\vec{q} / \omega$. The amplitude $\mathcal{M}_{\beta_{c}^{-} \gamma}$ and its hermitian conjugate are determined by

$$
\mathcal{M}_{\beta_{c}^{-} \gamma}=\left[\varphi_{p}^{\dagger} \varphi_{n}\right]\left[\bar{u}_{e} Q \gamma^{0}\left(1-\gamma^{5}\right) v_{\bar{\nu}}\right]-\lambda\left[\varphi_{p}^{\dagger} \vec{\sigma} \varphi_{n}\right] \cdot\left[\bar{u}_{e} Q \vec{\gamma}\left(1-\gamma^{5}\right) v_{\bar{\nu}}\right],
$$

and

$$
\mathcal{M}_{\beta_{c}^{-} \gamma}^{\dagger}=\left[\varphi_{n}^{\dagger} \varphi_{p}\right]\left[\bar{v}_{\bar{\nu}} \gamma^{0} \bar{Q}\left(1-\gamma^{5}\right) u_{e}\right]-\lambda\left[\varphi_{n}^{\dagger} \vec{\sigma} \varphi_{p}\right] \cdot\left[\bar{v}_{\bar{\nu}} \vec{\gamma} \bar{Q}\left(1-\gamma^{5}\right) u_{e}\right]
$$

where $Q=2\left(\varepsilon^{*} \cdot k_{e}\right)+\hat{\varepsilon}^{*} \hat{q}$ and $\bar{Q}=\gamma^{0} Q^{\dagger} \gamma^{0}=2\left(\varepsilon \cdot k_{e}\right)+\hat{q} \hat{\varepsilon}$.

The squared absolute value of the amplitude Eq.(B-4), summed up over the polarisations of the proton and the electron in the final state accounting for the polarization of the neutron is given by

$$
\begin{aligned}
& \sum_{\text {pol. }}\left|\mathcal{M}_{\beta_{c}^{-} \gamma}\right|^{2}=\operatorname{tr}\left\{\left(1+\vec{\xi}_{n} \cdot \vec{\sigma}\right)\right\} \operatorname{tr}\left\{\hat{k}_{e} Q \gamma^{0} \hat{k} \gamma^{0} \bar{Q}\left(1-\gamma^{5}\right)\right\}-\lambda \operatorname{tr}\left\{\left(1+\vec{\xi}_{n} \cdot \vec{\sigma}\right) \vec{\sigma}\right\} \cdot \operatorname{tr}\left\{\hat{k}_{e} Q \gamma^{0} \hat{k} \vec{\gamma} \bar{Q}\left(1-\gamma^{5}\right)\right\} \\
& -\lambda \operatorname{tr}\left\{\left(1+\vec{\xi}_{n} \cdot \vec{\sigma}\right) \vec{\sigma}\right\} \cdot \operatorname{tr}\left\{\hat{k}_{e} Q \vec{\gamma} \hat{k} \gamma^{0} \bar{Q}\left(1-\gamma^{5}\right)\right\}+\lambda^{2} \operatorname{tr}\left\{\left(1+\vec{\xi}_{n} \cdot \vec{\sigma}\right) \sigma^{m} \sigma^{n}\right\} \operatorname{tr}\left\{\hat{k}_{e} Q \gamma^{n} \hat{k} \gamma^{m} \bar{Q}\left(1-\gamma^{5}\right)\right\}
\end{aligned}
$$

Calculating the traces over the nucleon degrees of freedom and using the properties of the Dirac matrices

$$
\gamma^{\alpha} \gamma^{\nu} \gamma^{\mu}=\gamma^{\alpha} g^{\nu \mu}-\gamma^{\nu} g^{\mu \alpha}+\gamma^{\mu} g^{\alpha \nu}+i \varepsilon^{\alpha \nu \mu \beta} \gamma_{\beta} \gamma^{5}
$$

where $\varepsilon^{\alpha \nu \mu \beta}$ is the Levi-Civita tensor defined by $\varepsilon^{0123}=1$ and $\varepsilon_{\alpha \nu \mu \beta}=-\varepsilon^{\alpha \nu \mu \beta}$ [35], we transcribe the r.h.s. of Eq. (B-6) into the form

$$
\begin{aligned}
\sum_{\text {pol. }}\left|\mathcal{M}_{\beta_{c}^{-} \gamma}\right|^{2} & =2 E\left[\left(\left(1+3 \lambda^{2}\right)-2 \lambda(1-\lambda) \frac{\vec{\xi}_{n} \cdot \vec{k}}{E}\right) \operatorname{tr}\left\{\hat{k}_{e} Q \gamma^{0} \bar{Q}\left(1-\gamma^{5}\right)\right\}\right. \\
& \left.+\left(\left(1-\lambda^{2}\right) \frac{\vec{k}}{E}-2 \lambda(1+\lambda) \vec{\xi}_{n}\right) \cdot \operatorname{tr}\left\{\hat{k}_{e} Q \vec{\gamma} \bar{Q}\left(1-\gamma^{5}\right)\right\}\right] .
\end{aligned}
$$

The traces in Eq.(B-8) are equal to

$$
\begin{aligned}
& \frac{1}{16} \operatorname{tr}\left\{\hat{k}_{e} Q \gamma^{\mu} \bar{Q}\left(1-\gamma^{5}\right)\right\}=\left(\varepsilon^{*} \cdot k_{e}\right)\left(\varepsilon \cdot k_{e}\right)\left(k_{e}+q\right)^{\mu}-\frac{1}{2}\left(\varepsilon^{*} \cdot \varepsilon\right)\left(\left(k_{e} \cdot q\right) q^{\mu}-\frac{1}{2} q^{2} k_{e}^{\mu}\right) \\
& \left.-\frac{1}{2}\left(\varepsilon^{*} \cdot k_{e}\right) \varepsilon^{\mu}+\left(\varepsilon \cdot k_{e}\right) \varepsilon^{* \mu}\right)\left(k_{e} \cdot q+\frac{1}{2} q^{2}\right)+\frac{1}{2} i \varepsilon^{\mu \alpha \beta \nu}\left(\left(\varepsilon^{*} \cdot k_{e}\right) \varepsilon_{\alpha}-\left(\varepsilon \cdot k_{e}\right) \varepsilon_{\alpha}^{*}\right) q_{\beta} k_{e \nu} \\
& +\frac{1}{2} i\left(q^{\mu} q_{\rho}-\frac{1}{2} q^{2} g_{\rho}^{\mu}\right) \varepsilon^{\rho \alpha \beta \nu} \varepsilon_{\alpha}^{*} \varepsilon_{\beta} k_{e \nu},
\end{aligned}
$$


where $q^{2}=0$ for a real transverse photon. Summing up over the photon polarisations we obtain the following photon-electron energy and angular distribution of the radiative $\beta^{-}$-decay of the neutron

$$
\begin{aligned}
& \frac{d^{8} \lambda_{\beta_{c}^{-} \gamma}\left(E_{e}, \omega, \vec{k}_{e}, \vec{k}, \vec{q}, \vec{\xi}_{n}\right)}{d \omega d E_{e} d \Omega_{\vec{k}_{e}} d \Omega_{\vec{k}} d \Omega_{\vec{n}}}=\frac{\alpha}{2 \pi}\left(1+3 \lambda^{2}\right) \frac{G_{F}^{2}\left|V_{u d}\right|^{2}}{(2 \pi)^{6}} \sqrt{E_{e}^{2}-m_{e}^{2}} E_{e} F\left(E_{e}, Z=1\right)\left(E_{0}-E_{e}-\omega\right)^{2} \frac{1}{\omega} \\
& \times\left\{\left(1+B_{0} \frac{\vec{\xi}_{n} \cdot \vec{k}}{E}\right)\left(\frac{k_{e}^{2}-\left(\vec{n} \cdot \vec{k}_{e}\right)^{2}}{\left(E_{e}-\vec{n} \cdot \vec{k}_{e}\right)^{2}}\left(1+\frac{\omega}{E_{e}}\right)+\frac{1}{E_{e}-\vec{n} \cdot \vec{k}_{e}} \frac{\omega^{2}}{E_{e}}\right)\right. \\
& \left.\left.+\left(a_{0} \frac{\vec{k}}{E}+A_{0} \vec{\xi}_{n}\right) \cdot\left[\left(\frac{k_{e}^{2}-\left(\vec{n} \cdot \vec{k}_{e}\right)^{2}}{\left(E_{e}-\vec{n} \cdot \vec{k}_{e}\right)^{2}}+\frac{\omega}{E_{e}-\vec{n} \cdot \vec{k}_{e}}\right) \frac{\vec{k}_{e}}{E_{e}}+\left(-\frac{m_{e}^{2}}{\left(E_{e}-\vec{n} \cdot \vec{k}_{e}\right)^{2}}+\frac{E_{e}+\omega}{E_{e}-\vec{n} \cdot \vec{k}_{e}}\right) \frac{\vec{q}}{E_{e}}\right)\right]\right\} .
\end{aligned}
$$

For the unpolarised neutron Eq.(B-10) coincides with the spectrum, adduced in [47] (see Eq.(3) of Ref.[47] and a comment in [102]).

After the integration over the directions of the photon momentum the photon-electron energy and angular distribution takes the form

$$
\begin{aligned}
\frac{d^{6} \lambda_{\beta_{c}^{-} \gamma}\left(E_{e}, \omega, \vec{k}_{e}, \vec{k}, \vec{q}, \vec{\xi}_{n}\right)}{d \omega d E_{e} d \Omega_{\vec{k}_{e}} d \Omega_{\vec{k}}}= & \frac{\alpha}{\pi}\left(1+3 \lambda^{2}\right) \frac{G_{F}^{2}\left|V_{u d}\right|^{2}}{(2 \pi)^{5}} \sqrt{E_{e}^{2}-m_{e}^{2}} E_{e} F\left(E_{e}, Z=1\right)\left(E_{0}-E_{e}-\omega\right)^{2} \frac{1}{\omega} \\
& \times\left\{\left(1+B_{0} \frac{\vec{\xi}_{n} \cdot \vec{k}}{E}\right)\left\{\left(1+\frac{\omega}{E_{e}}+\frac{1}{2} \frac{\omega^{2}}{E_{e}^{2}}\right)\left[\frac{1}{\beta} \ln \left(\frac{1+\beta}{1-\beta}\right)-2\right]+\frac{\omega^{2}}{E_{e}^{2}}\right\}\right. \\
& \left.+\left(a_{0} \frac{\vec{k} \cdot \vec{k}_{e}}{E E_{e}}+A_{0} \frac{\vec{\xi}_{n} \cdot \vec{k}_{e}}{E_{e}}\right)\left[1+\frac{1}{\beta^{2}} \frac{\omega}{E_{e}}\left(1+\frac{1}{2} \frac{\omega}{E_{e}}\right)\right]\left[\frac{1}{\beta} \ln \left(\frac{1+\beta}{1-\beta}\right)-2\right]\right\} .
\end{aligned}
$$

Integrating over the phase volume of the final state we obtain the rate of the radiative $\beta^{-}$-decay of the neutron

$$
\begin{aligned}
& \lambda_{\beta_{c}^{-} \gamma}\left(\omega_{\min }\right)=\frac{\alpha}{\pi}\left(1+3 \lambda^{2}\right) \frac{G_{F}^{2}\left|V_{u d}\right|^{2}}{2 \pi^{3}} \int_{m_{e}}^{E_{0}-\omega_{\min }} d E_{e} \sqrt{E_{e}^{2}-m_{e}^{2}} E_{e} F\left(E_{e}, Z=1\right) \\
& \times \int_{\omega_{\min }}^{E_{0}-E_{e}} \frac{d \omega}{\omega}\left(E_{0}-E_{e}-\omega\right)^{2}\left\{\left(1+\frac{\omega}{E_{e}}+\frac{1}{2} \frac{\omega^{2}}{E_{e}^{2}}\right)\left[\frac{1}{\beta} \ln \left(\frac{1+\beta}{1-\beta}\right)-2\right]+\frac{\omega^{2}}{E_{e}^{2}}\right\} .
\end{aligned}
$$

The lowest photon energy $\omega_{\min }$ may be treated as the photon energy threshold of the detector.

For the photon energy interval $\omega_{\min } \leq \omega \leq \omega_{\max }$ the rate of the radiative $\beta^{-}$-decay of the neutron reads

$$
\begin{aligned}
\lambda_{\beta_{c}^{-} \gamma}\left(\omega_{\max }, \omega_{\min }\right)= & \frac{\alpha}{\pi}\left(1+3 \lambda^{2}\right) \frac{G_{F}^{2}\left|V_{u d}\right|^{2}}{2 \pi^{3}} \int_{\omega_{\min }}^{\omega_{\max }} \frac{d \omega}{\omega} \int_{m_{e}}^{E_{0}-\omega} d E_{e} \sqrt{E_{e}^{2}-m_{e}^{2}} E_{e} F\left(E_{e}, Z=1\right)\left(E_{0}-E_{e}-\omega\right)^{2} \\
& \times\left\{\left(1+\frac{\omega}{E_{e}}+\frac{1}{2} \frac{\omega^{2}}{E_{e}^{2}}\right)\left[\frac{1}{\beta} \ln \left(\frac{1+\beta}{1-\beta}\right)-2\right]+\frac{\omega^{2}}{E_{e}^{2}}\right\} .
\end{aligned}
$$

For the region of photon energies $15 \mathrm{keV} \leq \omega \leq 340 \mathrm{keV}$, used in the experiments by [46, 47] , the branching ratio is equal to $\mathrm{BR}_{\beta_{c}^{-} \gamma}=2.87 \times 10^{-3}$. This result agrees well with the experimental values $\mathrm{BR}_{\beta_{c}^{-} \gamma}=3.13(35) \times 10^{-3}$ and $\mathrm{BR}_{\beta_{c}^{-} \gamma}=3.09(32) \times 10^{-3}$ and the result $\mathrm{BR}_{\beta_{c}^{-} \gamma}=2.85 \times 10^{-3}$, calculated by Gardner [46, 47].

For the comparison with the analysis of the radiative $\beta^{-}$-decay, carried out by Bernard et al. [50], we calculate the branching ratio for the photon energy region $5 \mathrm{keV} \leq \omega \leq E_{0}-m_{e}$. The result $\mathrm{BR}_{\beta_{c}^{-} \gamma}=4.45 \times 10^{-3}$ is in a agreement with $\mathrm{BR}_{\beta_{c}^{-} \gamma}=4.41 \times 10^{-3}$, calculated in [50].

For the electron-energy and angular distribution of the radiative $\beta^{-}$-decay of the neutron we obtain the following expression

$$
\begin{aligned}
& \frac{d^{5} \lambda_{\beta_{c}^{-} \gamma}\left(E_{e}, \vec{k}_{e}, \vec{k}, \vec{\xi}_{n}, \omega_{\min }\right)}{d E_{e} d \Omega_{\vec{k}_{e}} d \Omega_{\vec{k}}}=\frac{\alpha}{\pi}\left(1+3 \lambda^{2}\right) \frac{G_{F}^{2}\left|V_{u d}\right|^{2}}{(2 \pi)^{5}}\left(E_{0}-E_{e}\right)^{2} \sqrt{E_{e}^{2}-m_{e}^{2}} E_{e} F\left(E_{e}, Z=1\right) \\
& \times\left\{\left(1+B_{0} \frac{\vec{\xi}_{n} \cdot \vec{k}}{E}\right) g_{\beta_{c}^{-} \gamma}^{(1)}\left(E_{e}, \omega_{\min }\right)+\left(a_{0} \frac{\vec{k} \cdot \vec{k}_{e}}{E E_{e}}+A_{0} \frac{\vec{\xi}_{n} \cdot \vec{k}_{e}}{E_{e}}\right) g_{\beta_{c}^{-} \gamma}^{(2)}\left(E_{e}, \omega_{\min }\right)\right\},
\end{aligned}
$$

where the functions $g_{\beta_{c}^{-} \gamma}^{(1)}\left(E_{e}, \omega_{\min }\right)$ and $g_{\beta_{c}^{-} \gamma}^{(2)}\left(E_{e}, \omega_{\min }\right)$ are defined by

$$
g_{\beta_{c}^{-} \gamma}^{(1)}\left(E_{e}, \omega_{\min }\right)=\int_{\omega_{\min }}^{E_{0}-E_{e}} \frac{d \omega}{\omega} \frac{\left(E_{0}-E_{e}-\omega\right)^{2}}{\left(E_{0}-E_{e}\right)^{2}}\left\{\left(1+\frac{\omega}{E_{e}}+\frac{1}{2} \frac{\omega^{2}}{E_{e}^{2}}\right)\left[\frac{1}{\beta} \ln \left(\frac{1+\beta}{1-\beta}\right)-2\right]+\frac{\omega^{2}}{E_{e}^{2}}\right\}=
$$




$$
\begin{aligned}
& =\left[2 \ln \left(\frac{E_{0}-E_{e}}{\omega_{\min }}\right)-3+\frac{2}{3} \frac{E_{0}-E_{e}}{E_{e}}\left(1+\frac{1}{8} \frac{E_{0}-E_{e}}{E_{e}}\right)\right]\left[\frac{1}{2 \beta} \ln \left(\frac{1+\beta}{1-\beta}\right)-1\right]+\frac{1}{12} \frac{\left(E_{0}-E_{e}\right)^{2}}{E_{e}^{2}}, \\
& g_{\beta_{c}^{-} \gamma}^{(2)}\left(E_{e}, \omega_{\min }\right)=\int_{\omega_{\min }}^{E_{0}-E_{e}} \frac{d \omega}{\omega} \frac{\left(E_{0}-E_{e}-\omega\right)^{2}}{\left(E_{0}-E_{e}\right)^{2}}\left[1+\frac{1}{\beta^{2}} \frac{\omega}{E_{e}}\left(1+\frac{1}{2} \frac{\omega}{E_{e}}\right)\right]\left[\frac{1}{\beta} \ln \left(\frac{1+\beta}{1-\beta}\right)-2\right]= \\
& =\left[2 \ln \left(\frac{E_{0}-E_{e}}{\omega_{\min }}\right)-3+\frac{2}{3} \frac{E_{0}-E_{e}}{\beta^{2} E_{e}}\left(1+\frac{1}{8} \frac{E_{0}-E_{e}}{E_{e}}\right)\right]\left[\frac{1}{2 \beta} \ln \left(\frac{1+\beta}{1-\beta}\right)-1\right] .
\end{aligned}
$$

We would like to note that as we show below the functions $g_{\beta_{c}^{-} \gamma}^{(1)}\left(E_{e}, \omega_{\min }\right)$ and $g_{\beta_{c}^{-} \gamma}^{(2)}\left(E_{e}, \omega_{\min }\right)$, calculated by means of the infrared cut-off regularization $\omega_{\text {min }}$, have energy dependencies different in comparison with the functions, calculated in the FPM regularization (see also [25]).

The discrepancy between the electron-energy and angular distributions, obtained for a real photon emission with the infrared cut-off and FPM regularization, respectively, we discuss first in terms of the logarithmically divergent integral, which defines the infrared divergent contribution to the amplitude of the radiative $\beta^{-}-$decay of the neutron. This integral is

$$
J(\beta)=\int \frac{d^{3} q}{4 \pi q_{0}} \frac{\beta^{2}-(\vec{v} \cdot \vec{\beta})^{2}}{\left(q_{0}-\vec{q} \cdot \vec{\beta}\right)^{2}},
$$

where $q_{0}=\sqrt{\vec{q}^{2}+\mu^{2}}$ is an energy of a photon with mass $\mu, \vec{\beta}=\vec{k}_{e} / E_{e}$ and $\vec{v}=\vec{q} / q_{0}$ are the velocities of the electron and massive photon, respectively. The region of the integration in Eq. (B-16) is restricted by $q_{1} \leq q \leq q_{2}$, where $q=|\vec{q}|$.

For the first time the integral Eq.(B-16) has been discussed by Kinoshita and Sirlin in [15]. It is obvious that replacing the lower and upper limits of the integral by $\omega_{\min }$ and $E_{0}-E_{e}$, respectively, and setting $\mu$ zero in the integrand the function $J(\beta)$, given by

$$
J(\beta)=2 \ln \left(\frac{E_{0}-E_{e}}{\omega_{\min }}\right)\left[\frac{1}{2 \beta} \ln \left(\frac{1+\beta}{1-\beta}\right)-1\right],
$$

defines the infrared divergent part of the functions $g_{\beta_{c}^{-} \gamma}^{(1)}\left(E_{e}, \omega_{\min }\right)$ and $g_{\beta_{c}^{-} \gamma}^{(2)}\left(E_{e}, \omega_{\min }\right)$ in Eq. (B-15).

Now let us calculate the integral Eq.(B-16) for $\mu \neq 0$. Following Kinoshita and Sirlin [15], we may rewrite the integral Eq.(B-16) as follows

$$
J\left(\beta ; q_{2}, q_{1}\right)=\frac{\beta^{2}}{2} \int_{-1}^{+1} d x \int_{q_{1}}^{q_{2}} \frac{d q q^{2}}{q_{0}^{3}} \frac{1-v^{2} x^{2}}{(1-\beta v x)^{2}}
$$

Making a change of variables $q \rightarrow v$, proposed by Kinoshita and Sirlin [15], we arrive at the integral

$$
J\left(\beta ; q_{2}, q_{1}\right)=\frac{\beta^{2}}{2} \int_{-1}^{+1} d x \int_{v_{1}}^{v_{2}} \frac{d v v^{2}}{1-v^{2}} \frac{1-v^{2} x^{2}}{(1-\beta v x)^{2}}
$$

Integrating over $x$

$$
J\left(\beta ; q_{2}, q_{1}\right)=\int_{v_{1}}^{v_{2}}\left[1+\frac{1}{1-\beta^{2} v^{2}}-\frac{2}{1-v^{2}}+\frac{1}{\beta} \frac{v}{1-v^{2}} \ln \left(\frac{1+\beta v}{1-\beta v}\right)\right] d v
$$

and over $v$ we obtain

$$
\begin{aligned}
& J\left(\beta ; q_{2}, q_{1}\right)=\ln \left[\frac{\left(1-v_{1}\right)}{\left(1-v_{2}\right)} \frac{\left(1+v_{2}\right)}{\left(1+v_{1}\right)}\right]\left[\frac{1}{2 \beta} \ln \left(\frac{1+\beta}{1-\beta}\right)-1\right]+\left(v_{2}-v_{1}\right)+\frac{1}{2 \beta} \ln \left[\frac{\left(1+\beta v_{2}\right)}{\left(1-\beta v_{2}\right)} \frac{\left(1-\beta v_{1}\right)}{\left(1+\beta v_{1}\right)}\right] \\
& -\frac{1}{2 \beta} L\left(\frac{\beta}{1+\beta}\left(1-v_{2}\right)\right)+\frac{1}{2 \beta} L\left(\frac{\beta}{1+\beta}\left(1-v_{1}\right)\right)+\frac{1}{2 \beta} L\left(-\frac{\beta}{1-\beta}\left(1-v_{2}\right)\right)-\frac{1}{2 \beta} L\left(-\frac{\beta}{1-\beta}\left(1-v_{1}\right)\right) \\
& +\frac{1}{2 \beta} L\left(\frac{\beta}{1+\beta}\left(1+v_{2}\right)\right)-\frac{1}{2 \beta} L\left(\frac{\beta}{1+\beta}\left(1+v_{1}\right)\right)-\frac{1}{2 \beta} L\left(-\frac{\beta}{1-\beta}\left(1+v_{2}\right)\right)+\frac{1}{2 \beta} L\left(-\frac{\beta}{1-\beta}\left(1+v_{1}\right)\right),
\end{aligned}
$$

where $L(x)$ is the Spence function, defined by [15, 103.

$$
L(x)=\int_{0}^{x} \frac{d t}{t} \ell n|1-t| .
$$


For $q_{1} \gg \mu$ the function $J(\beta)$ reduces to the form

$$
J\left(\beta ; q_{2}, q_{1}\right)=2 \ln \left(\frac{q_{2}}{q_{1}}\right)\left[\frac{1}{2 \beta} \ln \left(\frac{1+\beta}{1-\beta}\right)-1\right] .
$$

One may see that non-trivial finite and energy-dependent terms appear in the function $J(\beta)$, given by Eq. (B-21), for $q_{1}=0$ and the integration over the region $0 \leq q \leq q_{2}$. To show this we set $q_{1}=0$ and $q_{2}=q_{\max }$ in Eq. (B-18) and arrive at the expression

$$
J\left(\beta ; q_{\max }, 0\right)=2 \ln \left(\frac{2 q_{\max }}{\mu}\right)\left[\frac{1}{2 \beta} \ln \left(\frac{1+\beta}{1-\beta}\right)-1\right]+1+\frac{1}{2 \beta} \ln \left(\frac{1+\beta}{1-\beta}\right)+\frac{1}{2 \beta}\left[L\left(\frac{2 \beta}{1+\beta}\right)-L\left(-\frac{2 \beta}{1-\beta}\right)\right] .
$$

Due to the relation between Spence's functions [19, 21, 103]

$$
L\left(\frac{2 \beta}{1+\beta}\right)-L\left(-\frac{2 \beta}{1-\beta}\right)=2 L\left(\frac{2 \beta}{1+\beta}\right)-\frac{1}{2} \ln ^{2}\left(\frac{1+\beta}{1-\beta}\right)
$$

we derive the following expression for $J(\beta)$

$$
J\left(\beta ; q_{2}, q_{1}\right)=2 \ln \left(\frac{2 q_{\max }}{\mu}\right)\left[\frac{1}{2 \beta} \ln \left(\frac{1+\beta}{1-\beta}\right)-1\right]+1+\frac{1}{2 \beta} \ln \left(\frac{1+\beta}{1-\beta}\right)-\frac{1}{4 \beta} \ln ^{2}\left(\frac{1+\beta}{1-\beta}\right)+\frac{1}{\beta} L\left(\frac{2 \beta}{1+\beta}\right) .
$$

In turn, using the relation [19, 21, 103]

$$
L\left(\frac{2 \beta}{1+\beta}\right)=L(\beta)-L(-\beta)+\frac{1}{2}\left[L\left(\frac{1-\beta}{2}\right)-L\left(\frac{1+\beta}{2}\right)\right]+\frac{1}{2} \ln \left(\frac{1+\beta}{2}\right) \ln \left(\frac{1+\beta}{1-\beta}\right)
$$

we reduce Eq.(B-26) to the form, obtained by Kinoshita and Sirlin (see Eq. (C-4) of [15]).

As a result, the functions $g_{\beta_{c}^{-} \gamma}^{(1)}\left(E_{e}, \mu\right)$ and $g_{\beta_{c}^{-} \gamma}^{(2)}\left(E_{e}, \mu\right)$, having the form

$$
\begin{aligned}
& g_{\beta_{c}^{-} \gamma}^{(1)}\left(E_{e}, \mu\right)=\left[2 \ln \left(\frac{2\left(E_{0}-E_{e}\right)}{\mu}\right)-3+\frac{2}{3} \frac{E_{0}-E_{e}}{E_{e}}\left(1+\frac{1}{8} \frac{E_{0}-E_{e}}{E_{e}}\right)\right]\left[\frac{1}{2 \beta} \ln \left(\frac{1+\beta}{1-\beta}\right)-1\right]+1 \\
& +\frac{1}{12} \frac{\left(E_{0}-E_{e}\right)^{2}}{E_{e}^{2}}+\frac{1}{2 \beta} \ln \left(\frac{1+\beta}{1-\beta}\right)-\frac{1}{4 \beta} \ln ^{2}\left(\frac{1+\beta}{1-\beta}\right)+\frac{1}{\beta} L\left(\frac{2 \beta}{1+\beta}\right), \\
& g_{\beta_{c}^{-} \gamma}^{(2)}\left(E_{e}, \mu\right)=\left[2 \ln \left(\frac{2\left(E_{0}-E_{e}\right)}{\mu}\right)-3+\frac{2}{3} \frac{E_{0}-E_{e}}{\beta^{2} E_{e}}\left(1+\frac{1}{8} \frac{E_{0}-E_{e}}{E_{e}}\right)\right]\left[\frac{1}{2 \beta} \ln \left(\frac{1+\beta}{1-\beta}\right)-1\right]+1 \\
& +\frac{1}{2 \beta} \ln \left(\frac{1+\beta}{1-\beta}\right)-\frac{1}{4 \beta} \ln ^{2}\left(\frac{1+\beta}{1-\beta}\right)+\frac{1}{\beta} L\left(\frac{2 \beta}{1+\beta}\right)
\end{aligned}
$$

agree fully with the functions, obtained in 25] (see Eqs.(12) and (13) of Ref. 25]). We note that the same result, given by Eq. (B-26), for the logarithmically divergent integral Eq. (B-16) may be obtained within the dimensional regularization [21]. We will use the functions Eq.(B-28) for the calculation of the radiative corrections to the rate and the correlation coefficients of the $\beta^{-}$-decay of the neutron (see Appendix D).

\section{Appendix C: Analysis of infrared divergences of radiative corrections to continuum-state $\beta^{-}-$decay of neutron}

The radiative corrections, caused by one-virtual photon exchanges, are described by three irreducible diagrams. They are shown in Fig. 3. The diagrams in Figs. 3a and 3b define the self-energy corrections to the masses and wave functions of the proton and electron [13]- 18], respectively. As has been shown in [13]- 18], the self--energy corrections to the masses my be removed by the mass renormalisation, whereas the contributions of the self-energy diagrams to the wave functions cannot be removed fully by renormalisation of the wave functions of the proton and electron and give some observable terms. We calculate the contributions of the self-energy diagrams in Appendix D. In this Appendix we analyse the contribution of the vertex diagram in Fig. 3c.

The contribution of the vertex diagram to the amplitude of the continuum-state $\beta^{-}-$decay of the neutron may be written as (see Appendix D)

$$
M^{(\gamma)}\left(n \rightarrow p e^{-} \bar{\nu}_{e}\right)=e^{2} \frac{G_{F}}{\sqrt{2}} V_{u d} \mathcal{M}^{(\gamma)}
$$



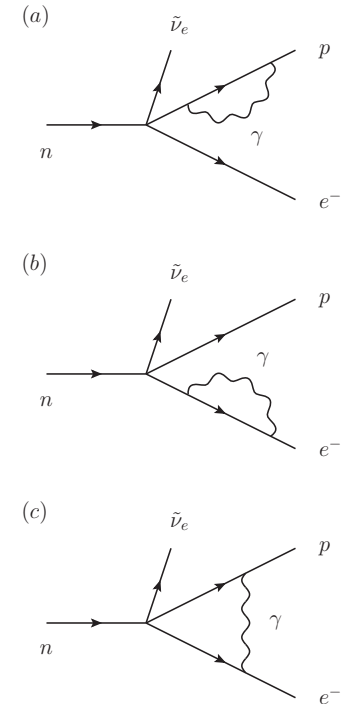

FIG. 3: Feynman diagrams of radiative corrections to the continuum-state $\beta^{-}$-decay of the neutron, caused by one-virtual photon exchanges.

The amplitude $\mathcal{M}^{(\gamma)}$ is defined by

$$
\mathcal{M}^{(\gamma)}=4\left(k_{e} \cdot k_{p}\right)\left[\bar{u}_{p} \gamma^{\mu}\left(1+\lambda \gamma^{5}\right) u_{n}\right]\left[\bar{u}_{e} \gamma_{\mu}\left(1-\gamma^{5}\right) v_{\bar{\nu}}\right] \int \frac{d^{4} q}{(2 \pi)^{4} i} \frac{1}{\left[q^{2}+i 0\right]\left[q^{2}-2 k_{e} \cdot q+i 0\right]\left[q^{2}+2 k_{p} \cdot q+i 0\right]}+\ldots,
$$

where we have kept only the term, which suffers from the infrared divergences. The detailed calculation of the vertex diagram in Fig. 3c is given in Appendix D.

For the calculation of the integral over the 4-momentum $q$ we follow the standard procedure, using Feynman's parametrization of the integrals [13, 18] (see also [104]). We perform the calculation of the 4-momentum integral in Eq. (C-2) by using the finite-photon mass regularization [13]- 18].

\section{A. finite-photon mass regularization}

Below we calculate the integral in Eq. (C-2) by using the finite-photon mass (FPM) regularization of the infrared divergences. Applying Feynman's parametrization [104] we transform the integral in Eq. (C-2) into the form

$$
J\left(E_{e} ; \mu\right)=\frac{1}{16 \pi^{2}} \int_{0}^{1} d x \int_{0}^{1} d y 2 y \int \frac{d^{4} q}{\pi^{2} i} \frac{1}{\left[(q-p(x) y)^{2}-p^{2}(x) y^{2}-\mu^{2}(1-y)+i 0\right]^{3}},
$$

where $p(x)=k_{e} x-k_{p}(1-x)$ and $\mu$ is an infinitesimal photon mass, introduced for the infrared regularization of the 4-momentum integrals [18, 104]. For the derivation of Eq. (C-3) we have used the relation [104]

$$
\frac{1}{A_{e} A_{p}}=\int_{0}^{1} \frac{d x}{\left[A_{e} x+A_{p}(1-x)\right]^{2}} \quad, \quad \frac{1}{A^{2} B}=\int_{0}^{1} \frac{2 y d y}{[A y+B(1-y)]^{3}},
$$

where $A_{e}=Q^{2}-2 k_{e} \cdot Q+i 0, A_{p}=Q^{2}+2 k_{p} \cdot Q+i 0, A=Q^{2}-2 p(x) \cdot Q+i 0$ and $B=Q^{2}-\mu^{2}+i 0$, respectively. Making a shift of variables $q-p(x) y \rightarrow q$, a Wick rotation $q_{0} \rightarrow i q_{4}$ [104] and integrating over $y$ we arrive at the expression [18, 19]

$$
J\left(E_{e} ; \mu\right)=-\frac{1}{32 \pi^{2}} \int_{0}^{1} \frac{d x}{p^{2}(x)} \ln \left[\frac{p^{2}(x)}{\mu^{2}}\right]
$$

where $p^{2}(x)=m_{e}^{2} x^{2}+m_{p}^{2}(1-x)^{2}-2 m_{e} m_{p} \gamma x(1-x)$ with $\gamma=1 / \sqrt{1-\beta^{2}}$. The r.h.s. of Eq. C-5 may be represented in the form

$$
J\left(E_{e} ; \mu\right)=-\frac{1}{32 \pi^{2}} \frac{1}{m_{e} m_{p} c} \int_{0}^{1} \frac{d x}{(a-x)^{2}-b^{2}} \ln \left[\frac{m_{e} m_{p} c}{\mu^{2}}\left((a-x)^{2}-b^{2}\right)\right],
$$


where we have denoted

$$
a=\frac{\rho+\gamma}{c}, b=\frac{\sqrt{\gamma^{2}-1}}{c}, c=\frac{1}{\rho}+\rho+2 \gamma
$$

with $\rho=m_{p} / m_{e}$. Following then [18, 19], we make a change of variables $a-x=b \operatorname{coth} \varphi$. This gives

$$
\begin{aligned}
& J\left(E_{e} ; \mu\right)=-\frac{1}{32 \pi^{2}} \frac{1}{m_{e} m_{p} b c} \int_{\varphi_{1}}^{\varphi_{2}} d \varphi \ln \left[\frac{m_{e} m_{p} c}{\mu^{2}} \frac{b^{2}}{\sinh ^{2} \varphi}\right]= \\
& =-\frac{1}{32 \pi^{2}} \frac{1}{m_{e} m_{p} b c}\left\{\ln \left[\frac{4 m_{e} m_{p} c}{\mu^{2}} b^{2}\right]\left(\varphi_{2}-\varphi_{1}\right)-\left(\varphi_{2}^{2}-\varphi_{1}^{2}\right)-2 \int_{\varphi_{1}}^{\varphi_{2}} d \varphi \ln \left(1-e^{-2 \varphi}\right)\right\},
\end{aligned}
$$

where $\varphi_{2}$ and $\varphi_{1}$ are equal to

$$
\begin{aligned}
& \varphi_{2}=\frac{1}{2} \ln \left(\frac{1-a-b}{1-a+b}\right)=\frac{1}{2} \ln \left(\frac{\frac{1}{\rho}+\gamma-\sqrt{\gamma^{2}-1}}{\frac{1}{\rho}+\gamma+\sqrt{\gamma^{2}-1}}\right), \\
& \varphi_{1}=\frac{1}{2} \ln \left(\frac{a+b}{a-b}\right)=\frac{1}{2} \ln \left(\frac{\rho+\gamma+\sqrt{\gamma^{2}-1}}{\rho+\gamma-\sqrt{\gamma^{2}-1}}\right) .
\end{aligned}
$$

For the calculation of the last integral in Eq. (C-8) we make a change of variables $\varphi=-\frac{1}{2} \ell$ lnt. This gives

$$
J(\beta ; \mu)=-\frac{1}{32 \pi^{2}} \frac{1}{m_{e} m_{p} b c}\left\{\ln \left[\frac{4 m_{e} m_{p} c}{\mu^{2}} b^{2}\right]\left(\varphi_{2}-\varphi_{1}\right)-\left(\varphi_{2}^{2}-\varphi_{1}^{2}\right)+L\left(e^{-2 \varphi_{2}}\right)-L\left(e^{-2 \varphi_{1}}\right)\right\},
$$

where the last two terms are the Spence functions, defined by Eq. (B-22).

Keeping the leading order contributions in the large $\rho$ expansion we arrive at the expression

$$
\begin{aligned}
J\left(E_{e} ; \mu\right) & =-\frac{1}{32 \pi^{2}} \frac{1}{m_{e} m_{p} \sqrt{\gamma^{2}-1}}\left\{-\ln \left[\frac{4 m_{e}^{2}}{\mu^{2}}\left(\gamma^{2}-1\right)\right] \frac{1}{2} \ln \left(\frac{\gamma+\sqrt{\gamma^{2}-1}}{\gamma-\sqrt{\gamma^{2}-1}}\right)-\frac{1}{4} \ln ^{2}\left(\frac{\gamma+\sqrt{\gamma^{2}-1}}{\gamma-\sqrt{\gamma^{2}-1}}\right)\right. \\
& \left.+L\left(\frac{\gamma+\sqrt{\gamma^{2}-1}}{\gamma-\sqrt{\gamma^{2}-1}}\right)-L(1)\right\} .
\end{aligned}
$$

In terms of the electron velocity $\beta$ it reads

$$
J(\beta ; \mu)=\frac{1}{32 \pi^{2}} \frac{1}{E_{e} m_{p} \beta}\left\{\ln \left[\frac{4 m_{e}^{2}}{\mu^{2}} \frac{\beta^{2}}{1-\beta^{2}}\right] \frac{1}{2} \ln \left(\frac{1+\beta}{1-\beta}\right)+\frac{1}{4} \ln ^{2}\left(\frac{1+\beta}{1-\beta}\right)-L\left(\frac{1+\beta}{1-\beta}\right)+L(1)\right\},
$$

where $E_{e}=m_{e} / \sqrt{1-\beta^{2}}$. For further transformation of the r.h.s. of Eq. (C-12) we use the following relation for the Spence functions 103 ]

$$
L\left(\frac{1+\beta}{1-\beta}\right)-L(1)=L\left(\frac{2 \beta}{1+\beta}\right)+\ln \left(\frac{2 \beta}{1-\beta}\right) \ln \left(\frac{1+\beta}{1-\beta}\right)-\frac{1}{2} \ln ^{2}\left(\frac{1+\beta}{1-\beta}\right) .
$$

Substituting Eq. (C-13) into Eq. (C-12) we obtain

$$
J(\beta ; \mu)=\frac{1}{32 \pi^{2}} \frac{1}{E_{e} m_{p} \beta}\left\{\ln \left(\frac{m_{e}}{\mu}\right) \ln \left(\frac{1+\beta}{1-\beta}\right)+\frac{1}{4} \ln ^{2}\left(\frac{1+\beta}{1-\beta}\right)-L\left(\frac{2 \beta}{1+\beta}\right)\right\} .
$$

Thus, the integral under consideration is equal to

$$
\begin{aligned}
& J\left(E_{e} ; \mu\right)=\int \frac{d^{4} q}{(2 \pi)^{4} i} \frac{1}{\left[q^{2}+i 0\right]\left[q^{2}-2 k_{e} \cdot q+i 0\right]\left[q^{2}+2 k_{p} \cdot q+i 0\right]} \\
& =\frac{1}{32 \pi^{2}} \frac{1}{E_{e} m_{p} \beta}\left\{\ln \left(\frac{m_{e}}{\mu}\right) \ln \left(\frac{1+\beta}{1-\beta}\right)+\frac{1}{4} \ln ^{2}\left(\frac{1+\beta}{1-\beta}\right)-L\left(\frac{2 \beta}{1+\beta}\right)\right\} .
\end{aligned}
$$

This result agrees with the expression, obtained in [18] and [19] (see Eq.(B21) of Ref. [19]). 


\section{Appendix D: Total contribution of one-virtual photon exchanges to continuum-state $\beta^{-}-$decay of neutron}

The contributions of one-virtual photon exchanges to the continuum-state $\beta^{-}$-decay of the neutron are shown in Fig. 3. The correction to the amplitude of the continuum-state $\beta^{-}$-decay of the neutron, caused by one-virtual photon exchanges, we represent in the following form

$$
M^{(\gamma)}\left(n \rightarrow p e^{-} \bar{\nu}_{e}\right)=-\frac{G_{F}}{\sqrt{2}} V_{u d}\left(\mathcal{M}_{p p}^{(\gamma)}+\mathcal{M}_{e e}^{(\gamma)}+\mathcal{M}_{p e}^{(\gamma)}\right)
$$

where the amplitudes $\mathcal{M}_{p p}^{(\gamma)}$ and $\mathcal{M}_{e e}^{(\gamma)}$ define the contributions of the self-energy diagrams of the proton and electron in Fig. 3a and Fig. 3b, respectively, and $\mathcal{M}_{p e}^{(\gamma)}$ is defined by the vertex diagram in Fig. 3c. Following [19] we calculate the diagrams in Fig. 3 in the Feynman gauge. As a result they are determined by the following analytical expressions

$$
\begin{aligned}
& \mathcal{M}_{p p}^{(\gamma)}=e^{2} \int \frac{d^{4} q}{(2 \pi)^{4} i} \frac{1}{q^{2}+i 0}\left[\bar{u}_{p} \gamma^{\alpha} \frac{1}{m_{p}-\hat{k}_{p}+\hat{q}-i 0} \gamma_{\alpha} \frac{1}{m_{p}-\hat{k}_{p}-i 0} \gamma^{\mu}\left(1+\lambda \gamma^{5}\right) u_{n}\right]\left[\bar{u}_{e} \gamma_{\mu}\left(1-\gamma^{5}\right) v_{\bar{\nu}}\right] \\
& +\left[\bar{u}_{p}\left(-\delta m_{p}+\frac{Z_{2}^{(p)}-1}{2}\left(m_{p}-\hat{k}_{p}\right)\right) \frac{1}{m_{p}-\hat{k}_{p}-i 0} \gamma^{\mu}\left(1+\lambda \gamma^{5}\right) u_{n}\right]\left[\bar{u}_{e} \gamma_{\mu}\left(1-\gamma^{5}\right) v_{\bar{\nu}}\right] \\
& \mathcal{M}_{e e}^{(\gamma)}=e^{2}\left[\bar{u}_{p} \gamma^{\mu}\left(1+\lambda \gamma^{5}\right) u_{n}\right] \int \frac{d^{4} q}{(2 \pi)^{4} i} \frac{1}{q^{2}+i 0}\left[\bar{u}_{e} \gamma^{\alpha} \frac{1}{m_{e}-\hat{k}_{e}-\hat{q}-i 0} \gamma_{\alpha} \frac{1}{m_{e}-\hat{k}_{e}-i 0} \gamma_{\mu}\left(1-\gamma^{5}\right) v_{\bar{\nu}}\right] \\
& +\left[\bar{u}_{p} \gamma^{\mu}\left(1+\lambda \gamma^{5}\right) u_{n}\right]\left[\bar{u}_{e}\left(-\delta m_{e}+\frac{Z_{2}^{(e)}-1}{2}\left(m_{e}-\hat{k}_{e}\right)\right) \frac{1}{m_{e}-\hat{k}_{e}-i 0} \gamma_{\mu}\left(1-\gamma^{5}\right) v_{\bar{\nu}}\right] \\
& \mathcal{M}_{p e}^{(\gamma)}=-e^{2} \int \frac{d^{4} q}{(2 \pi)^{4} i} \frac{1}{q^{2}+i 0}\left[\bar{u}_{p} \gamma^{\alpha} \frac{1}{m_{p}-\hat{k}_{p}+\hat{q}-i 0} \gamma^{\mu}\left(1+\lambda \gamma^{5}\right) u_{n}\right]\left[\bar{u}_{e} \gamma_{\alpha} \frac{1}{m_{e}-\hat{k}_{e}-\hat{q}-i 0} \gamma_{\mu}\left(1-\gamma^{5}\right) v_{\bar{\nu}}\right],(D)
\end{aligned}
$$

where $\left(\delta m_{p}, Z_{2}^{(p)}\right)$ and $\left(\delta m_{e}, Z_{2}^{(e)}\right)$ are the renormalisation constants of the masses and wave functions of the proton and electron, respectively.

The dependence on the electron energy is defined by the amplitude $\mathcal{M}_{p e}^{(\gamma)}$ only. For the calculation of $\mathcal{M}_{p e}^{(\gamma)}$ we reduce it to the form

$$
\begin{aligned}
\mathcal{M}_{p e}^{(\gamma)}= & -\frac{\alpha}{4 \pi} \int \frac{d^{4} q}{\pi^{2} i} \frac{1}{q^{2}+i 0} \frac{1}{q^{2}-2 k_{p} \cdot q+i 0} \frac{1}{q^{2}+2 k_{e} \cdot q+i 0} \\
& \times\left[\bar{u}_{p} \gamma^{\alpha}\left(m_{p}+\hat{k}_{p}-\hat{q}\right) \gamma^{\mu}\left(1+\lambda \gamma^{5}\right) u_{n}\right]\left[\bar{u}_{e} \gamma_{\alpha}\left(m_{e}+\hat{k}_{e}+\hat{q}\right) \gamma_{\mu}\left(1-\gamma^{5}\right) v_{\bar{\nu}}\right]
\end{aligned}
$$

where we have set $e^{2}=4 \pi \alpha$. The numerator of the integrand of $\mathcal{M}_{p e}^{(\gamma)}$ we transcribe into the form

$$
\begin{aligned}
& {\left[\bar{u}_{p} \gamma^{\alpha}\left(m_{p}+\hat{k}_{p}-\hat{q}\right) \gamma^{\mu}\left(1+\lambda \gamma^{5}\right) u_{n}\right]\left[\bar{u}_{e} \gamma_{\alpha}\left(m_{e}+\hat{k}_{e}+\hat{q}\right) \gamma_{\mu}\left(1-\gamma^{5}\right) v_{\bar{\nu}}\right]=} \\
& \left.=\left[\bar{u}_{p}\left(\left(m_{p}-\hat{k}_{p}+\hat{q}\right) \gamma^{\alpha}+2\left(k_{p}-q\right)^{\alpha}\right)\right) \gamma^{\mu}\left(1+\lambda \gamma^{5}\right) u_{n}\right]\left[\bar{u}_{e}\left(\left(m_{e}-\hat{k}_{e}-\hat{q}\right) \gamma_{\alpha}+2\left(k_{e}+q\right)_{\alpha}\right) \gamma_{\mu}\left(1-\gamma^{5}\right) v_{\bar{\nu}}\right]= \\
& \left.=\left[\bar{u}_{p}\left(\hat{q} \gamma^{\alpha}+2\left(k_{p}-q\right)^{\alpha}\right)\right) \gamma^{\mu}\left(1+\lambda \gamma^{5}\right) u_{n}\right]\left[\bar{u}_{e}\left(-\hat{q} \gamma_{\alpha}+2\left(k_{e}+q\right)_{\alpha}\right) \gamma_{\mu}\left(1-\gamma^{5}\right) v_{\bar{\nu}}\right]= \\
& =-\left[\bar{u}_{p} \hat{q} \gamma^{\alpha} \gamma^{\mu}\left(1+\lambda \gamma^{5}\right) u_{n}\right]\left[\bar{u}_{e} \hat{q} \gamma_{\alpha} \gamma_{\mu}\left(1-\gamma^{5}\right) v_{\bar{\nu}}\right]+\left[\bar{u}_{p} 2 \hat{q}\left(\hat{k}_{e}+\hat{q}\right) \gamma_{\mu}\left(1+\lambda \gamma^{5}\right) u_{n}\right]\left[\bar{u}_{e} \gamma_{\mu}\left(1-\gamma^{5}\right) v_{\bar{\nu}}\right] \\
& -\left[\bar{u}_{p} \gamma_{\mu}\left(1+\lambda \gamma^{5}\right) u_{n}\right]\left[\bar{u}_{e} 2 \hat{q}\left(\hat{k}_{p}-\hat{q}\right) \gamma_{\mu}\left(1-\gamma^{5}\right) v_{\bar{\nu}}\right]+4\left(k_{e}+q\right) \cdot\left(k_{p}-q\right)\left[\bar{u}_{p} \gamma^{\mu}\left(1+\lambda \gamma^{5}\right) u_{n}\right]\left[\bar{u}_{e} \gamma_{\mu}\left(1-\gamma^{5}\right) v_{\bar{\nu}}\right]= \\
& =-\left[\bar{u}_{p} \hat{q} \gamma^{\alpha} \gamma^{\mu}\left(1+\lambda \gamma^{5}\right) u_{n}\right]\left[\bar{u}_{e} \hat{q} \gamma_{\alpha} \gamma_{\mu}\left(1-\gamma^{5}\right) v_{\bar{\nu}}\right]+2 q^{2}\left[\bar{u}_{p} \gamma^{\mu}\left(1+\lambda \gamma^{5}\right) u_{n}\right]\left[\bar{u}_{e} \gamma_{\mu}\left(1-\gamma^{5}\right) v_{\bar{\nu}}\right] \\
& +\left(q^{2}+2 k_{e} \cdot q\right)\left[\bar{u}_{p} \gamma^{\mu}\left(1+\lambda \gamma^{5}\right) u_{n}\right]\left[\bar{u}_{e} \gamma_{\mu}\left(1-\gamma^{5}\right) v_{\bar{\nu}}\right]+\left(q^{2}-2 k_{p} \cdot q\right)\left[\bar{u}_{p} \gamma^{\mu}\left(1+\lambda \gamma^{5}\right) u_{n}\right]\left[\bar{u}_{e} \gamma_{\mu}\left(1-\gamma^{5}\right) v_{\bar{\nu}}\right] \\
& -2 i\left[\bar{u}_{p} \sigma_{\alpha \beta} q^{\alpha} k_{e}^{\beta} \gamma^{\mu}\left(1+\lambda \gamma^{5}\right) u_{n}\right]\left[\bar{u}_{e} \gamma_{\mu}\left(1-\gamma^{5}\right) v_{\bar{\nu}}\right]+2 i\left[\bar{u}_{p} \gamma^{\mu}\left(1+\lambda \gamma^{5}\right) u_{n}\right]\left[\bar{u}_{e} \sigma_{\alpha \beta} q^{\alpha} k_{p}^{\beta} \gamma_{\mu}\left(1-\gamma^{5}\right) v_{\bar{\nu}}\right] \\
& -2\left(q^{2}+2 k_{e} \cdot q\right)\left[\bar{u}_{p} \gamma^{\mu}\left(1+\lambda \gamma^{5}\right) u_{n}\right]\left[\bar{u}_{e} \gamma_{\mu}\left(1-\gamma^{5}\right) v_{\bar{\nu}}\right]-2\left(q^{2}-2 k_{p} \cdot q\right)\left[\bar{u}_{p} \gamma^{\mu}\left(1+\lambda \gamma^{5}\right) u_{n}\right]\left[\bar{u}_{e} \gamma_{\mu}\left(1-\gamma^{5}\right) v_{\bar{\nu}}\right] \\
& +4\left(k_{e} \cdot k_{p}\right)\left[\bar{u}_{p} \gamma^{\mu}\left(1+\lambda \gamma^{5}\right) u_{n}\right]\left[\bar{u}_{e} \gamma_{\mu}\left(1-\gamma^{5}\right) v_{\bar{\nu}}\right]=-\left[\bar{u}_{p} \hat{q} \gamma^{\alpha} \gamma^{\mu}\left(1+\lambda \gamma^{5}\right) u_{n}\right]\left[\bar{u}_{e} \hat{q} \gamma_{\alpha} \gamma_{\mu}\left(1-\gamma^{5}\right) v_{\bar{\nu}}\right] \\
& +2 q^{2}\left[\bar{u}_{p} \gamma^{\mu}\left(1+\lambda \gamma^{5}\right) u_{n}\right]\left[\bar{u}_{e} \gamma_{\mu}\left(1-\gamma^{5}\right) v_{\bar{\nu}}\right]-2 i\left[\bar{u}_{p} \sigma_{\alpha \beta} q^{\alpha} k_{e}^{\beta} \gamma^{\mu}\left(1+\lambda \gamma^{5}\right) u_{n}\right]\left[\bar{u}_{e} \gamma_{\mu}\left(1-\gamma^{5}\right) v_{\bar{\nu}}\right] \\
& +2 i\left[\bar{u}_{p} \gamma^{\mu}\left(1+\lambda \gamma^{5}\right) u_{n}\right]\left[\bar{u}_{e} \sigma_{\alpha \beta} q^{\alpha} k_{p}^{\beta} \gamma_{\mu}\left(1-\gamma^{5}\right) v_{\bar{\nu}}\right]-\left(q^{2}+2 k_{e} \cdot q\right)\left[\bar{u}_{p} \gamma^{\mu}\left(1+\lambda \gamma^{5}\right) u_{n}\right]\left[\bar{u}_{e} \gamma_{\mu}\left(1-\gamma^{5}\right) v_{\bar{\nu}}\right] \\
& -\left(q^{2}-2 k_{p} \cdot q\right)\left[\bar{u}_{p} \gamma^{\mu}\left(1+\lambda \gamma^{5}\right) u_{n}\right]\left[\bar{u}_{e} \gamma_{\mu}\left(1-\gamma^{5}\right) v_{\bar{\nu}}\right]+4\left(k_{e} \cdot k_{p}\right)\left[\bar{u}_{p} \gamma^{\mu}\left(1+\lambda \gamma^{5}\right) u_{n}\right]\left[\bar{u}_{e} \gamma_{\mu}\left(1-\gamma^{5}\right) v_{\bar{\nu}}\right] \text {, }
\end{aligned}
$$

where we have used the identity $\gamma^{\alpha} \gamma^{\beta}=g^{\alpha \beta}-i \sigma^{\alpha \beta}$. For the transformation of the product

$$
-\left[\bar{u}_{p} \hat{q} \gamma^{\alpha} \gamma^{\mu}\left(1+\lambda \gamma^{5}\right) u_{n}\right]\left[\bar{u}_{e} \hat{q} \gamma_{\alpha} \gamma_{\mu}\left(1-\gamma^{5}\right) v_{\bar{\nu}}\right]
$$


we propose to use the relations

$$
\begin{aligned}
& \hat{q} \gamma^{\alpha} \gamma^{\mu}=\gamma^{\mu} q^{\alpha}-\gamma^{\alpha} q^{\mu}+\hat{q} g^{\alpha \mu}+i \varepsilon^{\beta \alpha \mu \lambda} \gamma_{\lambda} \gamma^{5} q_{\beta} \\
& \varepsilon^{\beta \alpha \mu \lambda} \varepsilon_{\rho \alpha \mu \varphi} q_{\beta} q^{\rho}=-2 q^{2} g_{\varphi}^{\lambda}+2 q^{\lambda} q_{\varphi} \\
& \lambda+\gamma^{5}=-\left(1+\lambda \gamma^{5}\right)+(\lambda+1)\left(1+\gamma^{5}\right)
\end{aligned}
$$

This gives

$$
\begin{aligned}
& -\left[\bar{u}_{p} \hat{q} \gamma^{\alpha} \gamma^{\mu}\left(1+\lambda \gamma^{5}\right) u_{n}\right]\left[\bar{u}_{e} \hat{q} \gamma_{\alpha} \gamma_{\mu}\left(1-\gamma^{5}\right) v_{\bar{\nu}}\right]=-4 q^{2}\left[\bar{u}_{p} \gamma^{\mu}\left(1+\lambda \gamma^{5}\right) u_{n}\right]\left[\bar{u}_{e} \gamma_{\mu}\left(1-\gamma^{5}\right) v_{\bar{\nu}}\right] \\
& -2(\lambda+1)\left[\bar{u}_{p} \hat{q}\left(1+\gamma^{5}\right) u_{n}\right]\left[\bar{u}_{e} \hat{q}\left(1-\gamma^{5}\right) v_{\bar{\nu}}\right]+2(\lambda+1) q^{2}\left[\bar{u}_{p} \gamma^{\mu}\left(1+\gamma^{5}\right) u_{n}\right]\left[\bar{u}_{e} \gamma_{\mu}\left(1-\gamma^{5}\right) v_{\bar{\nu}}\right] .
\end{aligned}
$$

Substituting Eq. (D-6) into Eq. (D-4) we arrive at the following expression of the numerator of the integrand of $\mathcal{M}_{p e}^{(\gamma)}$ in Eq. (D-3)

$$
\begin{aligned}
& {\left[\bar{u}_{p} \gamma^{\alpha}\left(m_{p}+\hat{k}_{p}-\hat{q}\right) \gamma^{\mu}\left(1+\lambda \gamma^{5}\right) u_{n}\right]\left[\bar{u}_{e} \gamma_{\alpha}\left(m_{e}+\hat{k}_{e}+\hat{q}\right) \gamma_{\mu}\left(1-\gamma^{5}\right) v_{\bar{\nu}}\right]=} \\
& =-\left(q^{2}+2 k_{e} \cdot q\right)\left[\bar{u}_{p} \gamma^{\mu}\left(1+\lambda \gamma^{5}\right) u_{n}\right]\left[\bar{u}_{e} \gamma_{\mu}\left(1-\gamma^{5}\right) v_{\bar{\nu}}\right]-\left(q^{2}-2 k_{p} \cdot q\right)\left[\bar{u}_{p} \gamma^{\mu}\left(1+\lambda \gamma^{5}\right) u_{n}\right]\left[\bar{u}_{e} \gamma_{\mu}\left(1-\gamma^{5}\right) v_{\bar{\nu}}\right] \\
& -2 q^{2}\left[\bar{u}_{p} \gamma^{\mu}\left(1+\lambda \gamma^{5}\right) u_{n}\right]\left[\bar{u}_{e} \gamma_{\mu}\left(1-\gamma^{5}\right) v_{\bar{\nu}}\right]+4\left(k_{e} \cdot k_{p}\right)\left[\bar{u}_{p} \gamma^{\mu}\left(1+\lambda \gamma^{5}\right) u_{n}\right]\left[\bar{u}_{e} \gamma_{\mu}\left(1-\gamma^{5}\right) v_{\bar{\nu}}\right] \\
& -2(\lambda+1)\left[\bar{u}_{p} \hat{q}\left(1+\gamma^{5}\right) u_{n}\right]\left[\bar{u}_{e} \hat{q}\left(1-\gamma^{5}\right) v_{\bar{\nu}}\right]+2(\lambda+1) q^{2}\left[\bar{u}_{p} \gamma^{\mu}\left(1+\gamma^{5}\right) u_{n}\right]\left[\bar{u}_{e} \gamma_{\mu}\left(1-\gamma^{5}\right) v_{\bar{\nu}}\right] \\
& -2 i\left[\bar{u}_{p} \sigma_{\alpha \beta} q^{\alpha} k_{e}^{\beta} \gamma^{\mu}\left(1+\lambda \gamma^{5}\right) u_{n}\right]\left[\bar{u}_{e} \gamma_{\mu}\left(1-\gamma^{5}\right) v_{\bar{\nu}}\right]+2 i\left[\bar{u}_{p} \gamma^{\mu}\left(1+\lambda \gamma^{5}\right) u_{n}\right]\left[\bar{u}_{e} \sigma_{\alpha \beta} q^{\alpha} k_{p}^{\beta} \gamma_{\mu}\left(1-\gamma^{5}\right) v_{\bar{\nu}}\right] .
\end{aligned}
$$

For the subsequent calculations it is convenient to represent the amplitude $\mathcal{M}_{p e}^{(\gamma)}$ as a sum of two contributions

$$
\mathcal{M}_{p e}^{(\gamma)}=\overline{\mathcal{M}}_{p e}^{(\gamma)}+\delta \mathcal{M}_{p e}^{(\gamma)}
$$

where $\overline{\mathcal{M}}_{p e}^{(\gamma)}$ and $\delta \mathcal{M}_{p e}^{(\gamma)}$ are given by

$$
\begin{aligned}
& \overline{\mathcal{M}}_{p e}^{(\gamma)}=\frac{\alpha}{4 \pi}\left[\bar{u}_{p} \gamma^{\mu}\left(1+\lambda \gamma^{5}\right) u_{n}\right]\left[\bar{u}_{e} \gamma_{\mu}\left(1-\gamma^{5}\right) v_{\bar{\nu}}\right]\left\{\int \frac{d^{4} q}{\pi^{2} i} \frac{1}{q^{2}+i 0} \frac{1}{q^{2}-2 k_{p} \cdot q+i 0}+\int \frac{d^{4} q}{\pi^{2} i} \frac{1}{q^{2}+i 0} \frac{1}{q^{2}+2 k_{e} \cdot q+i 0}\right. \\
& \left.+2 \int \frac{d^{4} q}{\pi^{2} i} \frac{1}{q^{2}-2 k_{p} \cdot q+i 0} \frac{1}{q^{2}+2 k_{e} \cdot q+i 0}-4\left(k_{e} \cdot k_{p}\right) \int \frac{d^{4} q}{\pi^{2} i} \frac{1}{q^{2}+i 0} \frac{1}{q^{2}-2 k_{p} \cdot q+i 0} \frac{1}{q^{2}+2 k_{e} \cdot q+i 0}\right\} .
\end{aligned}
$$

and

$$
\begin{aligned}
& \delta \mathcal{M}_{p e}^{(\gamma)}=\delta^{(1)} \mathcal{M}_{p e}^{(\gamma)}+\delta^{(2)} \mathcal{M}_{p e}^{(\gamma)}+\delta^{(3)} \mathcal{M}_{p e}^{(\gamma)}= \\
& =\frac{\alpha}{4 \pi}\left\{-2(\lambda+1)\left[\bar{u}_{p} \gamma^{\mu}\left(1+\gamma^{5}\right) u_{n}\right]\left[\bar{u}_{e} \gamma_{\mu}\left(1-\gamma^{5}\right) v_{\bar{\nu}}\right] \int \frac{d^{4} q}{\pi^{2} i} \frac{1}{q^{2}-2 k_{p} \cdot q+i 0} \frac{1}{q^{2}+2 k_{e} \cdot q+i 0}\right. \\
& +2(\lambda+1) \int \frac{d^{4} q}{\pi^{2} i} \frac{1}{q^{2}+i 0} \frac{1}{q^{2}-2 k_{p} \cdot q+i 0} \frac{1}{q^{2}+2 k_{e} \cdot q+i 0}\left[\bar{u}_{p} \hat{q}\left(1+\gamma^{5}\right) u_{n}\right]\left[\bar{u}_{e} \hat{q}\left(1-\gamma^{5}\right) v_{\bar{\nu}}\right] \\
& +2 i \int \frac{d^{4} q}{\pi^{2} i} \frac{1}{q^{2}+i 0} \frac{1}{q^{2}-2 k_{p} \cdot q+i 0} \frac{1}{q^{2}+2 k_{e} \cdot q+i 0}\left(\left[\bar{u}_{p} \sigma_{\alpha \beta} q^{\alpha} k_{e}^{\beta} \gamma^{\mu}\left(1+\lambda \gamma^{5}\right) u_{n}\right]\left[\bar{u}_{e} \gamma_{\mu}\left(1-\gamma^{5}\right) v_{\bar{\nu}}\right]\right. \\
& \left.\left.-\left[\bar{u}_{p} \gamma^{\mu}\left(1+\lambda \gamma^{5}\right) u_{n}\right]\left[\bar{u}_{e} \sigma_{\alpha \beta} q^{\alpha} k_{p}^{\beta} \gamma_{\mu}\left(1-\gamma^{5}\right) v_{\bar{\nu}}\right]\right)\right\}
\end{aligned}
$$

respectively. Now let us proceed to calculate $\overline{\mathcal{M}}_{p e}^{\gamma}$, given by Eq. (D-9). Since, the last term in Eq.(D-9) is calculated in Appendix C, so we should calculate the first three terms only. Using the Pauli-Villars regularization for the ultra-violet divergent integrals 35

$$
\frac{1}{q^{2}+i 0} \rightarrow \frac{1}{q^{2}+i 0}-\frac{1}{q^{2}-\Lambda^{2}+i 0}=-\frac{1}{q^{2}+i 0} \frac{\Lambda^{2}}{q^{2}-\Lambda^{2}+i 0},
$$

where $\Lambda$ is an ultraviolet cut-off, then Feynman's unification of the denominators, the shift of the virtual 4-momentum and the Wick rotation [35] for the first three integrals in Eq. (D-9) we obtain the following expressions

$$
\begin{aligned}
& \int \frac{d^{4} q}{\pi^{2} i} \frac{1}{q^{2}+i 0} \frac{1}{q^{2}-2 k_{p} \cdot q+i 0} \rightarrow \int \frac{d^{4} q}{\pi^{2} i}\left[\frac{1}{q^{2}+i 0} \frac{1}{q^{2}-2 k_{p} \cdot q+i 0}-\frac{1}{q^{2}-\Lambda^{2}+i 0} \frac{1}{q^{2}-2 k_{p} \cdot q+i 0}\right]= \\
& =\int_{0}^{1} d x \int \frac{d^{4} q}{\pi^{2}}\left[\frac{1}{\left(q^{2}+m_{p}^{2} x^{2}\right)^{2}}-\frac{1}{\left(q^{2}+m_{p}^{2} x^{2}+\Lambda^{2}(1-x)\right)^{2}}\right]=\int_{0}^{1} d x \ln \left[\frac{\Lambda^{2}(1-x)}{m_{p}^{2} x^{2}}\right]=2 \ln \left(\frac{\Lambda}{m_{p}}\right)+1 \\
& \int \frac{d^{4} q}{\pi^{2} i} \frac{1}{q^{2}+i 0} \frac{1}{q^{2}+2 k_{e} \cdot q+i 0}=2 \ln \left(\frac{\Lambda}{m_{e}}\right)+1
\end{aligned}
$$




$$
\begin{aligned}
& \int \frac{d^{4} q}{\pi^{2} i} \frac{1}{q^{2}-2 k_{p} \cdot q+i 0} \frac{1}{q^{2}+2 k_{e} \cdot q+i 0}=\int \frac{d^{4} q}{\pi^{2} i} \frac{1}{q^{2}+i 0} \frac{q^{2}}{q^{2}-2 k_{p} \cdot q+i 0} \frac{1}{q^{2}+2 k_{e} \cdot q+i 0} \rightarrow \\
& \rightarrow \int_{0}^{1} d x \int_{0}^{1} d y 2 y \int \frac{d^{4} q}{\pi^{2} i}\left[\frac{q^{2}}{\left.(q-p(x) y)^{2}-p^{2}(x) y^{2}-\mu^{2}(1-y)\right)^{3}}-\frac{q^{2}}{\left.(q-p(x) y)^{2}-p^{2}(x) y^{2}-\Lambda^{2}(1-y)\right)^{3}}\right]= \\
& =\int_{0}^{1} d x \int_{0}^{1} d y 2 y \int \frac{d^{4} q}{\pi^{2}}\left[\frac{q^{2}-p^{2}(x) y^{2}}{\left(q^{2}+p^{2}(x) y^{2}+\mu^{2}(1-y)\right)^{3}}-\frac{1}{\left(q^{2}+p^{2}(x) y^{2}+\Lambda^{2}(1-y)\right)^{3}}\right]= \\
& =\int_{0}^{1} d x \int_{0}^{1} d y 2 y \int \frac{d^{4} q}{\pi^{2}}\left\{\frac{1}{\left(q^{2}+p^{2}(x) y^{2}+\mu^{2}(1-y)\right)^{2}}-\frac{2 p^{2}(x) y^{2}+\Lambda^{2}(1-y)}{\left(q^{2}+p^{2}(x) y^{2}+\Lambda^{2}(1-y)\right)^{2}}\right. \\
& \left.-\frac{2 p^{2}(x) y^{2}}{\left(q^{2}+p^{2}(x) y^{2}+\mu^{2}(1-y)\right)^{3}}+\frac{p^{2}}{\left(q^{2}+p^{2}(x) y^{2}+\Lambda^{2}(1-y)\right)^{3}}\right\}=\int_{0}^{1} d x \int_{0}^{1} d y 2 y\left\{\ln \left[\frac{\Lambda^{2}(1-y)}{p^{2}(x) y^{2}}\right]-\frac{1}{2}\right\}= \\
& =\int_{0}^{1} d x\left\{\ln \left[\frac{\Lambda^{2}}{p^{2}(x)}\right]-1\right\}=2 \ln \left(\frac{\Lambda}{m_{p}}\right)+1 .
\end{aligned}
$$

For the calculation of the third integral we have replaced $p^{2}(x)=\left(k_{e} x-k_{p}(1-x)\right)^{2}$ by $m_{p}^{2}(1-x)^{2}$. For the calculation of the amplitudes $\mathcal{M}_{e e}^{(\gamma)}$ and $\mathcal{M}_{p p}^{(\gamma)}$ we have to perform the following standard transformations

$$
\begin{aligned}
& \int \frac{d^{4} q}{\pi^{2} i} \bar{u} \frac{1}{q^{2}+i 0} \gamma^{\alpha} \frac{1}{\hat{k}+\hat{q}-m} \gamma_{\alpha}=\bar{u} \int_{0}^{1} d x \int \frac{d^{4} q}{\pi^{2} i} \frac{4 m-2 \hat{k}-2 \hat{q}}{\left[(q+k x)-m^{2} x+k^{2} x(1-x)\right]^{2}}= \\
& =\int_{0}^{1} d x \bar{u} \int \frac{d^{4} q}{\pi^{2}} \frac{4 m-2 \hat{k}(1-x)}{\left[q^{2}+m^{2} x-k^{2} x(1-x)\right]^{2}}=\int_{0}^{1} d x \bar{u} \int \frac{d^{4} q}{\pi^{2}} \frac{2 m(1+x)+2(1-x)(m-\hat{k})}{\left[q^{2}+m^{2} x-k^{2} x(1-x)\right]^{2}}= \\
& =\int_{0}^{1} d x \bar{u}\left[\int \frac{d^{4} q}{\pi^{2}} \frac{2 m(1+x)}{\left[q^{2}+m^{2} x-k^{2} x(1-x)\right]^{2}}+\frac{2(1-x)}{\left[q^{2}+m^{2} x-k^{2} x(1-x)\right]^{2}}(m-\hat{k})-\frac{4 m x\left(1-x^{2}\right)(m+\hat{k})}{\left[q^{2}+m_{e}^{2} x-k^{2} x(1-x)\right]^{3}}\right. \\
& \times(m-\hat{k})]=\bar{u}\left\{\int_{0}^{1} d x \int \frac{d^{4} q}{\pi^{2}} \frac{2 m(1+x)}{\left(q^{2}+m^{2} x^{2}\right)^{2}}+(m-\hat{k}) \int_{0}^{1} d x \int \frac{d^{4} q}{\pi^{2}}\left[\frac{2(1-x)}{\left(q^{2}+m^{2} x^{2}\right)^{2}}-\frac{8 m^{2} x\left(1-x^{2}\right)}{\left(q^{2}+m^{2} x^{2}\right)^{3}}\right]\right\} .
\end{aligned}
$$

This results in the relation

$$
\begin{aligned}
\int \frac{d^{4} q}{\pi^{2} i} \bar{u} \frac{1}{q^{2}+i 0} \gamma^{\alpha} \frac{1}{\hat{q}+\hat{k}-m} \gamma_{\alpha} & =\bar{u}\left\{\int_{0}^{1} d x \int \frac{d^{4} q}{\pi^{2}} \frac{2 m(1+x)}{\left(q^{2}+m^{2} x^{2}\right)^{2}}\right. \\
& \left.+(m-\hat{k}) \int_{0}^{1} d x \int \frac{d^{4} q}{\pi^{2}}\left[\frac{2(1-x)}{\left(q^{2}+m^{2} x^{2}\right)^{2}}-\frac{8 m^{2} x\left(1-x^{2}\right)}{\left(q^{2}+m^{2} x^{2}\right)^{3}}\right]\right\}
\end{aligned}
$$

where $m=m_{e}$ and $m=m_{p}$ for the electron and proton self-energy contributions, respectively. The term, which is not proportional to $(m-\hat{k})$, can be removed by the mass renormalisation. So the amplitudes $\mathcal{M}_{e e}^{(\gamma)}$ and $\mathcal{M}_{p p}^{(\gamma)}$ are defined by the contributions of the terms, proportional to $\left(m_{e}-\hat{k}_{e}\right)$ and $\left(m_{p}-\hat{k}_{p}\right)$, respectively. This gives

$$
\begin{aligned}
& \mathcal{M}_{e e}^{(\gamma)}=\left[\bar{u}_{p} \gamma^{\mu}\left(1+\lambda \gamma^{5}\right) u_{n}\right]\left[\bar{u}_{e} \gamma_{\mu}\left(1-\gamma^{5}\right) v_{\bar{\nu}}\right]\left(\frac{\alpha}{4 \pi} I\left(m_{e}\right)+\frac{Z_{2}^{(e)}-1}{2}\right), \\
& \mathcal{M}_{p p}^{(\gamma)}=\left[\bar{u}_{p} \gamma^{\mu}\left(1+\lambda \gamma^{5}\right) u_{n}\right]\left[\bar{u}_{e} \gamma_{\mu}\left(1-\gamma^{5}\right) v_{\bar{\nu}}\right]\left(\frac{\alpha}{4 \pi} I\left(m_{p}\right)+\frac{Z_{2}^{(p)}-1}{2}\right),
\end{aligned}
$$

where $I(m)$ is given by

$$
I(m)=\int_{0}^{1} d x \int \frac{d^{4} q}{\pi^{2}}\left[-\frac{2(1-x)}{\left(q^{2}+m^{2} x^{2}\right)^{2}}+\frac{8 m^{2} x\left(1-x^{2}\right)}{\left(q^{2}+m^{2} x^{2}+\mu^{2}(1-x)\right)^{3}}\right]
$$

for $m=m_{e}$ and $m_{p}$, respectively. For the ultra-violet and infrared regularization we use the following expressions for the photon Green function

$$
\frac{1}{q^{2}+i 0} \rightarrow \frac{1}{q^{2}-\mu^{2}+i 0}-\frac{1}{q^{2}-\Lambda^{2}+i 0}
$$

The regularized function $I(m)$ takes the form

$$
\begin{aligned}
I(m) & =\int_{0}^{1} d x \int \frac{d^{4} q}{\pi^{2}}\left[-\frac{2(1-x)}{\left(q^{2}+m^{2} x^{2}\right)^{2}}+\frac{2(1-x)}{\left(q^{2}+m^{2} x^{2}+\Lambda^{2}(1-x)\right)^{2}}\right. \\
& \left.+\frac{8 m^{2} x\left(1-x^{2}\right)}{\left(q^{2}+m^{2} x^{2}+\mu^{2}(1-x)\right)^{3}}-\frac{8 m^{2} x\left(1-x^{2}\right)}{\left(q^{2}+m^{2} x^{2}+\Lambda^{2}(1-x)\right)^{3}}\right] .
\end{aligned}
$$


The calculation of the integrals in Eq. (D-18) runs as follows. For the first two integrals, which are ultra-violet divergent, we obtain the expression

$$
\int_{0}^{1} d x \int \frac{d^{4} q}{\pi^{2}}\left[\frac{2(1-x)}{\left(q^{2}+m^{2} x^{2}+\Lambda^{2}(1-x)\right)^{2}}-\frac{2(1-x)}{\left(q^{2}+m^{2} x^{2}\right)^{2}}\right]=-\int_{0}^{1} d x 2(1-x) \ln \left[\frac{(1-x) \Lambda^{2}}{x^{2} m^{2}}\right]-2 \ln \left(\frac{\Lambda}{m}\right)-\frac{5}{2} .
$$

The third and fourth integrals in Eq.(D-18) are ultra-violet convergent. Moreover in the limit $\Lambda \rightarrow \infty$ the fourth integral vanishes. The third integral is infrared divergent and its calculation gives

$$
\int_{0}^{1} d x \int \frac{d^{4} q}{\pi^{2}} \frac{8 m^{2} x\left(1-x^{2}\right)}{\left(q^{2}+\mu^{2}(1-x)+m^{2} x^{2}\right)^{3}}=4 m^{2} \int_{0}^{1} d x \frac{x\left(1-x^{2}\right)}{\mu^{2}(1-x)+m^{2} x^{2}}=-4 \ln \left(\frac{\mu}{m}\right)-2 .
$$

Thus, the function $I(m)$ takes the form

$$
I(m)=-2 \ln \left(\frac{\Lambda}{m}\right)-4 \ln \left(\frac{\mu}{m}\right)-\frac{9}{2} .
$$

Summing up the contributions of the amplitudes $\mathcal{M}_{e e}^{(\gamma)}, \mathcal{M}_{p p}^{(\gamma)}$ and $\overline{\mathcal{M}}_{p e}^{(\gamma)}$ we obtain the following expression

$$
\begin{aligned}
& \mathcal{M}_{e e}^{(\gamma)}+\mathcal{M}_{p p}^{(\gamma)}+\overline{\mathcal{M}}_{p e}^{(\gamma)}=\left[\bar{u}_{p} \gamma^{\mu}\left(1+\lambda \gamma^{5}\right) u_{n}\right]\left[\bar{u}_{e} \gamma_{\mu}\left(1-\gamma^{5}\right) v_{\bar{\nu}}\right]\left\{\frac{Z_{2}^{(e)}-1}{2}+\frac{Z_{2}^{(p)}-1}{2}\right. \\
& +\frac{\alpha}{4 \pi}\left[-2 \ln \left(\frac{\Lambda}{m_{e}}\right)-4 \ln \left(\frac{\mu}{m_{e}}\right)-\frac{9}{2}-2 \ln \left(\frac{\Lambda}{m_{p}}\right)-4 \ln \left(\frac{\mu}{m_{p}}\right)-\frac{9}{2}+2 \ln \left(\frac{\Lambda}{m_{p}}\right)+1\right. \\
& \left.\left.+2 \ln \left(\frac{\Lambda}{m_{e}}\right)+1+4 \ln \left(\frac{\Lambda}{m_{p}}\right)+2+2\left\{\ln \left(\frac{\mu}{m_{e}}\right) \frac{1}{\beta} \ln \left(\frac{1+\beta}{1-\beta}\right)-\frac{1}{4 \beta} \ln ^{2}\left(\frac{1+\beta}{1-\beta}\right)+\frac{1}{\beta} L\left(\frac{2 \beta}{1+\beta}\right)\right\}\right]\right\}
\end{aligned}
$$

After the cancellation of some terms we arrive at the expression

$$
\begin{aligned}
& \mathcal{M}_{e e}^{(\gamma)}+\mathcal{M}_{p p}^{(\gamma)}+\overline{\mathcal{M}}_{p e}^{(\gamma)}=\left[\bar{u}_{p} \gamma^{\mu}\left(1+\lambda \gamma^{5}\right) u_{n}\right]\left[\bar{u}_{e} \gamma_{\mu}\left(1-\gamma^{5}\right) v_{\bar{\nu}}\right]\left\{\frac{Z_{2}^{(e)}-1}{2}+\frac{Z_{2}^{(p)}-1}{2}\right. \\
& \left.+\frac{\alpha}{2 \pi}\left[2 \ln \left(\frac{\Lambda}{m_{p}}\right)-2 \ln \left(\frac{\mu}{m_{e}}\right)-2 \ln \left(\frac{\mu}{m_{p}}\right)-\frac{5}{2}+\frac{1}{\beta} \ln \left(\frac{\mu}{m_{e}}\right) \ln \left(\frac{1+\beta}{1-\beta}\right)-\frac{1}{4 \beta} \ln ^{2}\left(\frac{1+\beta}{1-\beta}\right)+\frac{1}{\beta} L\left(\frac{2 \beta}{1+\beta}\right)\right]\right\} .
\end{aligned}
$$

Then, we transcribe the r.h.s. of Eq.(D-23) into the following symmetric form

$$
\begin{aligned}
& \mathcal{M}_{e e}^{(\gamma)}+\mathcal{M}_{p p}^{(\gamma)}+\overline{\mathcal{M}}_{p e}^{(\gamma)}=\left[\bar{u}_{p} \gamma^{\mu}\left(1+\lambda \gamma^{5}\right) u_{n}\right]\left[\bar{u}_{e} \gamma_{\mu}\left(1-\gamma^{5}\right) v_{\bar{\nu}}\right] \\
& \times\left\{\left[\frac{Z_{2}^{(e)}-1}{2}-\frac{\alpha}{2 \pi}\left(\frac{1}{2} \ln \left(\frac{\Lambda}{m_{e}}\right)+\ln \left(\frac{\mu}{m_{e}}\right)+\frac{9}{8}\right)\right]+\left[\frac{Z_{2}^{(p)}-1}{2}-\frac{\alpha}{2 \pi}\left(\frac{1}{2} \ln \left(\frac{\Lambda}{m_{p}}\right)+\ln \left(\frac{\mu}{m_{p}}\right)+\frac{9}{8}\right)\right]\right. \\
& \left.+\frac{\alpha}{2 \pi}\left[3 \ln \left(\frac{\Lambda}{m_{p}}\right)+\frac{3}{2} \ln \left(\frac{m_{p}}{m_{e}}\right)-\frac{1}{4}+2 \ln \left(\frac{\mu}{m_{e}}\right)\left[\frac{1}{2 \beta} \ln \left(\frac{1+\beta}{1-\beta}\right)-1\right]-\frac{1}{4 \beta} \ln \left(\frac{1+\beta}{1-\beta}\right)+\frac{1}{\beta} L\left(\frac{2 \beta}{1+\beta}\right)\right]\right\} .
\end{aligned}
$$

Defining the constants of the renormalisation of the wave functions of the electron and proton in the standard form as 35 .

$$
\begin{aligned}
& \frac{Z_{2}^{(e)}-1}{2}=\frac{\alpha}{2 \pi}\left[\frac{1}{2} \ln \left(\frac{\Lambda}{m_{e}}\right)+\ln \left(\frac{\mu}{m_{e}}\right)+\frac{9}{8}\right] \\
& \frac{Z_{2}^{(p)}-1}{2}=\frac{\alpha}{2 \pi}\left[\frac{1}{2} \ln \left(\frac{\Lambda}{m_{p}}\right)+\ln \left(\frac{\mu}{m_{p}}\right)+\frac{9}{8}\right]
\end{aligned}
$$

we reduce the r.h.s. of Eq. (D-24) to the form

$$
\begin{aligned}
& \mathcal{M}_{e e}^{(\gamma)}+\mathcal{M}_{p p}^{(\gamma)}+\overline{\mathcal{M}}_{p e}^{(\gamma)}=\left[\bar{u}_{p} \gamma^{\mu}\left(1+\lambda \gamma^{5}\right) u_{n}\right]\left[\bar{u}_{e} \gamma_{\mu}\left(1-\gamma^{5}\right) v_{\bar{\nu}}\right] \\
& \times \frac{\alpha}{2 \pi}\left\{3 \ln \left(\frac{\Lambda}{m_{p}}\right)+\frac{3}{2} \ln \left(\frac{m_{p}}{m_{e}}\right)-\frac{1}{4}+2 \ln \left(\frac{\mu}{m_{e}}\right)\left[\frac{1}{2 \beta} \ln \left(\frac{1+\beta}{1-\beta}\right)-1\right]+\frac{1}{\beta} L\left(\frac{2 \beta}{1+\beta}\right)-\frac{1}{4 \beta} \ln ^{2}\left(\frac{1+\beta}{1-\beta}\right)\right\} .
\end{aligned}
$$


In the non-relativistic approximation for the proton Eq. (D-26) reads

$$
\begin{aligned}
& \mathcal{M}_{e e}^{(\gamma)}+\mathcal{M}_{p p}^{(\gamma)}+\overline{\mathcal{M}}_{p e}^{(\gamma)}=\frac{\alpha}{2 \pi}\left\{3 \ln \left(\frac{\Lambda}{m_{p}}\right)+\frac{3}{2} \ln \left(\frac{m_{p}}{m_{e}}\right)-\frac{1}{4}+2 \ln \left(\frac{\mu}{m_{e}}\right)\left[\frac{1}{2 \beta} \ln \left(\frac{1+\beta}{1-\beta}\right)-1\right]\right. \\
& \left.+\frac{1}{\beta} L\left(\frac{2 \beta}{1+\beta}\right)-\frac{1}{4 \beta} \ln ^{2}\left(\frac{1+\beta}{1-\beta}\right)\right\}\left\{\left[\varphi_{p}^{\dagger} \varphi_{n}\right]\left[\bar{u}_{e} \gamma^{0}\left(1-\gamma^{5}\right) v_{\bar{\nu}}\right]-\lambda\left[\varphi_{p}^{\dagger} \vec{\sigma} \varphi_{n}\right] \cdot\left[\bar{u}_{e} \vec{\gamma}\left(1-\gamma^{5}\right) v_{\bar{\nu}}\right]\right\} .
\end{aligned}
$$

Now let us take into account the contribution of the first two terms in $\delta \mathcal{M}_{p e}^{(\gamma)}$, given by Eq. (D-10). The first term in Eq.(D-10) is equal to

$$
\begin{aligned}
& \delta^{(1)} \mathcal{M}_{p e}^{(\gamma)}=-(\lambda+1) \frac{\alpha}{2 \pi}\left[\bar{u}_{p} \gamma^{\mu}\left(1+\gamma^{5}\right) u_{n}\right]\left[\bar{u}_{e} \gamma_{\mu}\left(1-\gamma^{5}\right) v_{\bar{\nu}}\right] \int \frac{d^{4} q}{\pi^{2} i} \frac{1}{q^{2}-2 k_{p} \cdot q+i 0} \frac{1}{q^{2}+2 k_{e} \cdot q+i 0}= \\
& =-(\lambda+1) \frac{\alpha}{2 \pi}\left[2 \ell n\left(\frac{\Lambda}{m_{p}}\right)+1\right]\left[\bar{u}_{p} \gamma^{\mu}\left(1+\gamma^{5}\right) u_{n}\right]\left[\bar{u}_{e} \gamma_{\mu}\left(1-\gamma^{5}\right) v_{\bar{\nu}}\right]
\end{aligned}
$$

where we have used Eq. (D-12). In the non-relativistic approximation for the proton it reads

$$
\delta^{(1)} \mathcal{M}_{p e}^{(\gamma)}=-2 m_{n}(\lambda+1) \frac{\alpha}{2 \pi}\left[2 \ln \left(\frac{\Lambda}{m_{p}}\right)+1\right]\left\{\left[\varphi_{p}^{\dagger} \varphi_{n}\right]\left[\bar{u}_{e} \gamma^{0}\left(1-\gamma^{5}\right) v_{\bar{\nu}}\right]-\left[\varphi_{p}^{\dagger} \vec{\sigma} \varphi_{n}\right] \cdot\left[\bar{u}_{e} \vec{\gamma}\left(1-\gamma^{5}\right) v_{\bar{\nu}}\right]\right\}
$$

The calculation of the second term in Eq. (D-10) runs as follows

$$
\begin{aligned}
& \delta^{(2)} \mathcal{M}_{p e}^{(\gamma)}=(\lambda+1) \frac{\alpha}{2 \pi}\left[\bar{u}_{p} \gamma^{\alpha}\left(1+\gamma^{5}\right) u_{n}\right]\left[\bar{u}_{e} \gamma^{\beta}\left(1-\gamma^{5}\right) v_{\bar{\nu}}\right] \int \frac{d^{4} q}{\pi^{2} i} \frac{q_{\alpha} q_{\beta}}{q^{2}+i 0} \frac{1}{q^{2}-2 k_{p} \cdot q+i 0} \frac{1}{q^{2}+2 k_{e} \cdot q+i 0} \rightarrow \\
& \rightarrow(\lambda+1) \frac{\alpha}{2 \pi}\left[\bar{u}_{p} \gamma^{\alpha}\left(1+\gamma^{5}\right) u_{n}\right]\left[\bar{u}_{e} \gamma^{\beta}\left(1-\gamma^{5}\right) v_{\bar{\nu}}\right] \int \frac{d^{4} q}{\pi^{2} i}\left[\frac{q_{\alpha} q_{\beta}}{q^{2}+i 0}-\frac{q_{\alpha} q_{\beta}}{q^{2}-\Lambda^{2}+i 0}\right] \frac{1}{q^{2}-2 k_{p} \cdot q+i 0} \frac{1}{q^{2}+2 k_{e} \cdot q+i 0}= \\
& =(\lambda+1) \frac{\alpha}{2 \pi}\left[\bar{u}_{p} \gamma^{\alpha}\left(1+\gamma^{5}\right) u_{n}\right]\left[\bar{u}_{e} \gamma^{\beta}\left(1-\gamma^{5}\right) v_{\bar{\nu}}\right] \int_{0}^{1} d x \int_{0}^{1} d y 2 y \int \frac{d^{4} q}{\pi^{2} i}\left[\frac{q_{\beta}}{\left.(q+p(x) y)^{2}-p^{2}(x) y^{2}\right)^{3}}\right. \\
& \left.-\frac{q_{\alpha} q_{\beta}}{\left.(q+p(x) y)^{2}-p^{2}(x) y^{2}-\Lambda^{2}(1-y)\right)^{3}}\right] .
\end{aligned}
$$

After the shift of variables, the integration over the 4-dimensional solid angle and the Wick rotation we arrive at the expression

$$
\begin{aligned}
& \delta^{(2)} \mathcal{M}_{p e}^{(\gamma)}=(\lambda+1) \frac{\alpha}{8 \pi}\left[\bar{u}_{p} \gamma^{\alpha}\left(1+\gamma^{5}\right) u_{n}\right]\left[\bar{u}_{e} \gamma^{\beta}\left(1-\gamma^{5}\right) v_{\bar{\nu}}\right] \int_{0}^{1} d x \int_{0}^{1} d y 2 y \int \frac{d^{4} q}{\pi^{2}}\left[\frac{q^{2} g_{\alpha \beta}-4 y^{2} p_{\alpha}(x) p_{\beta}(x)}{\left(q^{2}+p^{2}(x) y^{2}\right)^{3}}\right. \\
& \left.-\frac{q^{2} g_{\alpha \beta}-4 y^{2} p_{\alpha}(x) p_{\beta}(x)}{\left(q^{2}+p^{2}(x) y^{2}+\Lambda^{2}(1-y)\right)^{3}}\right]=(\lambda+1) \frac{\alpha}{8 \pi}\left[\bar{u}_{p} \gamma^{\alpha}\left(1+\gamma^{5}\right) u_{n}\right]\left[\bar{u}_{e} \gamma^{\beta}\left(1-\gamma^{5}\right) v_{\bar{\nu}}\right]\left\{\int_{0}^{1} d x \int_{0}^{1} d y 2 y \int \frac{d^{4} q}{\pi^{2}} \frac{g_{\alpha \beta}}{\left(q^{2}+p^{2}(x) y^{2}\right)^{2}}\right. \\
& -\int_{0}^{1} d x \int_{0}^{1} d y 2 y^{3} \int \frac{d^{4} q}{\pi^{2}} \frac{p^{2}(x) g_{\alpha \beta}+4 p_{\alpha}(x) p_{\beta}(x)}{\left(q^{2}+p^{2}(x) y^{2}\right)^{3}}-\int_{0}^{1} d x \int_{0}^{1} d y 2 y \int \frac{d^{4} q}{\pi^{2}} \frac{g_{\alpha \beta}}{\left(q^{2}+p^{2}(x) y^{2}+\Lambda^{2}(1-y)\right)^{2}} \\
& \left.+\int_{0}^{1} d x \int_{0}^{1} d y 2 y \int \frac{d^{4} q}{\pi^{2}} \frac{\left(p^{2}(x) y^{2}+\Lambda^{2}(1-y)\right) g_{\alpha \beta}}{\left(q^{2}+p^{2}(x) y^{2}+\Lambda^{2}(1-y)\right)^{3}}+\int_{0}^{1} d x \int_{0}^{1} d y 2 y \int \frac{d^{4} q}{\pi^{2}} \frac{4 y^{2} p_{\alpha}(x) p_{\beta}(x)}{\left(q^{2}+p^{2}(x) y^{2}+\Lambda^{2}(1-y)\right)^{3}}\right\} .
\end{aligned}
$$

After the integration over $q$ the term $\delta^{(2)} \mathcal{M}_{p e}^{(\gamma)}$ is

$\delta^{(2)} \mathcal{M}_{p e}^{(\gamma)}=(\lambda+1) \frac{\alpha}{8 \pi}\left[\bar{u}_{p} \gamma^{\alpha}\left(1+\gamma^{5}\right) u_{n}\right]\left[\bar{u}_{e} \gamma^{\beta}\left(1-\gamma^{5}\right) v_{\bar{\nu}}\right]\left\{g_{\alpha \beta} \int_{0}^{1} d x \int_{0}^{1} d y 2 y \ln \left[\frac{\Lambda^{2}(1-y)}{p^{2}(x) y^{2}}\right]-2 \int_{0}^{1} d x \frac{p_{\alpha}(x) p_{\beta}(x)}{p^{2}(x)}\right\}$

Keeping the leading terms in the large $m_{p}$ expansion, i.e. replacing $p^{2}(x)=\left(k_{e} x-k_{p}(1-x)\right)^{2}$ by $p^{2}(x) \rightarrow m_{p}^{2}(1-x)^{2}$, and integrating over $y$ and $x$ we obtain

$$
\delta^{(2)} \mathcal{M}_{p e}^{(\gamma)}=(\lambda+1) \frac{\alpha}{4 \pi}\left[\bar{u}_{p} \gamma^{\alpha}\left(1+\gamma^{5}\right) u_{n}\right]\left[\bar{u}_{e} \gamma^{\beta}\left(1-\gamma^{5}\right) v_{\bar{\nu}}\right]\left\{g_{\alpha \beta}\left[\ln \left(\frac{\Lambda}{m_{p}}\right)+\frac{3}{4}\right]-g_{o \alpha} g_{0 \beta}\right\} .
$$


In the non-relativistic approximation for the proton Eq. (D-33) takes the form

$$
\begin{aligned}
& \delta^{(2)} \mathcal{M}_{p e}^{(\gamma)}=2 m_{n} \frac{\alpha}{2 \pi}\left\{(\lambda+1)\left[\frac{1}{2} \ln \left(\frac{\Lambda}{m_{p}}\right)+\frac{1}{4}\right]\right\}\left[\varphi_{p}^{\dagger} \varphi_{n}\right]\left[\bar{u}_{e} \gamma^{0}\left(1-\gamma^{5}\right) v_{\bar{\nu}}\right] \\
& +2 m_{n} \frac{\alpha}{2 \pi}\left\{(\lambda+1)\left[-\frac{1}{2} \ln \left(\frac{\Lambda}{m_{p}}\right)-\frac{3}{4}\right]\right\}\left[\varphi_{p}^{\dagger} \vec{\sigma} \varphi_{n}\right] \cdot\left[\bar{u}_{e} \vec{\gamma}\left(1-\gamma^{5}\right) v_{\bar{\nu}}\right] .
\end{aligned}
$$

The sum of the contributions of $\delta^{(1)} \mathcal{M}_{p e}^{(\gamma)}$ and $\delta^{(2)} \mathcal{M}_{p e}^{(\gamma)}$ is

$$
\begin{aligned}
\delta^{(1)} \mathcal{M}_{p e}^{(\gamma)}+\delta^{(2)} \mathcal{M}_{p e}^{(\gamma)} & =2 m_{n}\left\{\frac{\alpha}{2 \pi}(\lambda+1)\left[-\frac{3}{2} \ln \left(\frac{\Lambda}{m_{p}}\right)-\frac{3}{4}\right]\right\}\left[\varphi_{p}^{\dagger} \varphi_{n}\right]\left[\bar{u}_{e} \gamma^{0}\left(1-\gamma^{5}\right) v_{\bar{\nu}}\right] \\
& +2 m_{n}\left\{\frac{\alpha}{2 \pi}(\lambda+1)\left[+\frac{3}{2} \ln \left(\frac{\Lambda}{m_{p}}\right)+\frac{1}{4}\right]\right\}\left[\varphi_{p}^{\dagger} \vec{\sigma} \varphi_{n}\right] \cdot\left[\bar{u}_{e} \vec{\gamma}\left(1-\gamma^{5}\right) v_{\bar{\nu}}\right]
\end{aligned}
$$

Now we have to calculate the contribution of the last term $\delta^{(3)} \mathcal{M}_{p e}^{(\gamma)}$ to the amplitude $\delta \mathcal{M}_{p e}^{(\gamma)}$, given by

$$
\begin{aligned}
& \delta^{(3)} \mathcal{M}_{p e}^{(\gamma)}=\frac{\alpha}{2 \pi} \int \frac{d^{4} q}{\pi^{2} i} \frac{1}{q^{2}+i 0} \frac{1}{q^{2}-2 k_{p} \cdot q+i 0} \frac{1}{q^{2}+2 k_{e} \cdot q+i 0} \\
& \times\left(\left[\bar{u}_{p} i \sigma_{\alpha \beta} q^{\alpha} k_{e}^{\beta} \gamma^{\mu}\left(1+\lambda \gamma^{5}\right) u_{n}\right]\left[\bar{u}_{e} \gamma_{\mu}\left(1-\gamma^{5}\right) v_{\bar{\nu}}\right]-\left[\bar{u}_{p} \gamma^{\mu}\left(1+\lambda \gamma^{5}\right) u_{n}\right]\left[\bar{u}_{e} i \sigma_{\alpha \beta} q^{\alpha} k_{p}^{\beta} \gamma_{\mu}\left(1-\gamma^{5}\right) v_{\bar{\nu}}\right]\right) .
\end{aligned}
$$

Following the procedure expounded above, we reduce the r.h.s of Eq. (D-36) to the form

$$
\begin{aligned}
& \delta^{(3)} \mathcal{M}_{p e}^{(\gamma)}=\frac{\alpha}{2 \pi}\left\{\left[\bar{u}_{p} i \sigma_{\alpha \beta} k_{e}^{\alpha} k_{p}^{\beta} \gamma^{\mu}\left(1+\lambda \gamma^{5}\right) u_{n}\right]\left[\bar{u}_{e} \gamma_{\mu}\left(1-\gamma^{5}\right) v_{\bar{\nu}}\right] \int_{0}^{1} \frac{d x(1-x)}{p^{2}(x)}\right. \\
& \left.-\left[\bar{u}_{p} \gamma^{\mu}\left(1+\lambda \gamma^{5}\right) u_{n}\right]\left[\bar{u}_{e} i \sigma_{\alpha \beta} k_{e}^{\alpha} k_{p}^{\beta} \gamma_{\mu}\left(1-\gamma^{5}\right) v_{\bar{\nu}}\right] \int_{0}^{1} \frac{d x x}{p^{2}(x)}\right\}
\end{aligned}
$$

where $p^{2}(x)=\left(k_{e} x-k_{p}(1-x)\right)^{2}=m_{e}^{2} x^{2}+m_{p}^{2}(1-x)^{2}-2 m_{e} m_{p} \gamma x(1-x)$.

The calculation of the integrals over $x$ in Eq.(D-37) has been carried out in detail in Appendix C. Using these results we obtain

$$
\begin{aligned}
& \int_{0}^{1} \frac{d x}{p^{2}(x)}=-\frac{1}{m_{e} m_{p}} \frac{\sqrt{1-\beta^{2}}}{2 \beta} \ln \left(\frac{1+\beta}{1-\beta}\right) \\
& \int_{0}^{1} \frac{d x x}{p^{2}(x)}=-\frac{1}{m_{e} m_{p}} \frac{\sqrt{1-\beta^{2}}}{2 \beta} \ln \left(\frac{1+\beta}{1-\beta}\right) \\
& -\frac{1}{m_{p}^{2}}\left[\ln \left(\frac{m_{p}}{m_{e}}\right)-\frac{1}{2 \beta} \ln \left(\frac{1+\beta}{1-\beta}\right)\right]+\ldots \\
& \int_{0}^{1} \frac{d x(1-x)}{p^{2}(x)}=\frac{1}{m_{p}^{2}}\left[\ln \left(\frac{m_{p}}{m_{e}}\right)-\frac{1}{2 \beta} \ln \left(\frac{1+\beta}{1-\beta}\right)\right]+\ldots
\end{aligned}
$$

where the ellipses denote the contributions of the terms of higher order in the large $m_{p}$ expansion.

Keeping the leading terms in the large $m_{p}$ expansion, the contribution of $\delta^{(3)} \mathcal{M}_{p e}^{(\gamma)}$ is

$$
\delta^{(3)} \mathcal{M}_{p e}^{(\gamma)}=\frac{\alpha}{2 \pi}\left[\frac{\sqrt{1-\beta^{2}}}{2 \beta} \ln \left(\frac{1+\beta}{1-\beta}\right)\right]\left[\bar{u}_{p} \gamma^{\mu}\left(1+\lambda \gamma^{5}\right) u_{n}\right]\left[\bar{u}_{e} \frac{E_{e}-m_{e} \gamma^{0}}{m_{e}} \gamma_{\mu}\left(1-\gamma^{5}\right) v_{\bar{\nu}}\right],
$$

where we have used the Dirac equation for the free electron $\bar{u}_{e}\left(\vec{k}_{e} \cdot \vec{\gamma}\right)=\bar{u}_{e}\left(E_{e} \gamma^{0}-m_{e}\right)$. Thus $\delta^{(3)} \mathcal{M}_{p e}^{(\gamma)}$ is given by

$$
\begin{aligned}
\delta^{(3)} \mathcal{M}_{p e}^{(\gamma)} & =\frac{\alpha}{2 \pi}\left[\frac{1}{2 \beta} \ln \left(\frac{1+\beta}{1-\beta}\right)\right]\left[\bar{u}_{p} \gamma^{\mu}\left(1+\lambda \gamma^{5}\right) u_{n}\right]\left[\bar{u}_{e} \gamma_{\mu}\left(1-\gamma^{5}\right) v_{\bar{\nu}}\right] \\
& -\frac{\alpha}{2 \pi}\left[\frac{\sqrt{1-\beta^{2}}}{2 \beta} \ln \left(\frac{1+\beta}{1-\beta}\right)\right]\left[\bar{u}_{p} \gamma^{\mu}\left(1+\lambda \gamma^{5}\right) u_{n}\right]\left[\bar{u}_{e} \gamma^{0} \gamma_{\mu}\left(1-\gamma^{5}\right) v_{\bar{\nu}}\right]
\end{aligned}
$$


In the non-relativistic approximation for the proton Eq. (D-40) reads

$$
\begin{aligned}
& \delta^{(3)} \mathcal{M}_{p e}^{(\gamma)}=2 m_{n} \frac{\alpha}{2 \pi} \frac{1}{2 \beta} \ln \left(\frac{1+\beta}{1-\beta}\right)\left\{\left[\varphi_{p}^{\dagger} \varphi_{n}\right]\left[\bar{u}_{e} \gamma^{0}\left(1-\gamma^{5}\right) v_{\bar{\nu}}\right]-\lambda\left[\varphi_{p}^{\dagger} \vec{\sigma} \varphi_{n}\right] \cdot\left[\bar{u}_{e} \vec{\gamma}\left(1-\gamma^{5}\right) v_{\bar{\nu}}\right]\right\} \\
& -2 m_{n} \frac{\alpha}{2 \pi} \frac{\sqrt{1-\beta^{2}}}{2 \beta} \ln \left(\frac{1+\beta}{1-\beta}\right)\left\{\left[\varphi_{p}^{\dagger} \varphi_{n}\right]\left[\bar{u}_{e}\left(1-\gamma^{5}\right) v_{\bar{\nu}}\right]-\lambda\left[\varphi_{p}^{\dagger} \vec{\sigma} \varphi_{n}\right] \cdot\left[\bar{u}_{e} \gamma^{0} \vec{\gamma}\left(1-\gamma^{5}\right) v_{\bar{\nu}}\right]\right\} .
\end{aligned}
$$

The second term can be identified with the contributions of the scalar and tensor lepton-nucleon weak interactions. In order to show this we use the Hamilton density operator of weak interactions, taken in the following form [10]

$$
\begin{aligned}
\mathcal{H}_{W}(x) & =\frac{G_{F}}{\sqrt{2}} V_{u d}\left\{\left[\bar{\psi}_{p}(x) \gamma_{\mu}\left(1+\lambda \gamma^{5}\right) \psi_{n}(x)\right]\left[\bar{\psi}_{e}(x) \gamma^{\mu}\left(1-\gamma^{5}\right) \psi_{\nu_{e}}(x)\right]\right. \\
& \left.+g_{S}\left[\bar{\psi}_{p}(x) \psi_{n}(x)\right]\left[\bar{\psi}_{e}(x)\left(1-\gamma^{5}\right) \psi_{\nu_{e}}(x)\right]+\frac{1}{2} g_{T}\left[\bar{\psi}_{p}(x) \sigma_{\mu \nu} \gamma^{5} \psi_{n}(x)\right]\left[\bar{\psi}_{e}(x) \sigma^{\mu \nu}\left(1-\gamma^{5}\right) \psi_{\nu_{e}}(x)\right]\right\}
\end{aligned}
$$

where $g_{S}$ and $g_{T}$ are the constants of scalar and tensor weak interactions and $\sigma_{\mu \nu}=\frac{i}{2}\left(\gamma_{\mu} \gamma_{\nu}-\gamma_{\nu} \gamma_{\mu}\right)$ is the Dirac matrix.

In the rest frame of the neutron and in the non-relativistic approximation for the proton the amplitude of the continuum-state $\beta^{-}$-decay of the neutron takes the form [10]

$M\left(n \rightarrow p+e^{-}+\tilde{\nu}_{e}\right)=-2 m_{n} \frac{G_{F}}{\sqrt{2}} V_{u d}\left\{\left[\varphi_{p}^{\dagger} \varphi_{n}\right]\left[\bar{u}_{e}\left(1+g_{S} \gamma^{0}\right) \gamma^{0}\left(1-\gamma^{5}\right) v_{\bar{\nu}}\right]+\left[\varphi_{p}^{\dagger} \vec{\sigma} \varphi_{n}\right] \cdot\left[\bar{u}_{e}\left(-\lambda+g_{T} \gamma^{0}\right) \vec{\gamma}\left(1-\gamma^{5}\right) v_{\bar{\nu}}\right\}\right.$

From the comparison Eq.(D-41) with Eq.(D-43) we define the scalar and tensor coupling constants as

$$
\begin{aligned}
& g_{S}\left(E_{e}\right)=-\frac{\alpha}{2 \pi} \frac{\sqrt{1-\beta^{2}}}{2 \beta} \ln \left(\frac{1+\beta}{1-\beta}\right)=-\frac{\alpha}{2 \pi} g_{F}\left(E_{e}\right), \\
& g_{T}\left(E_{e}\right)=+\frac{\alpha}{2 \pi} \lambda \frac{\sqrt{1-\beta^{2}}}{2 \beta} \ln \left(\frac{1+\beta}{1-\beta}\right)=+\frac{\alpha}{2 \pi} \lambda g_{F}\left(E_{e}\right) .
\end{aligned}
$$

Using the results, obtained in [10], we may define the contribution of the electromagnetic Fierz term [10]

$$
b_{F}^{(\mathrm{em})}\left(E_{e}\right)=2 \frac{g_{S}\left(E_{e}\right)-3 \lambda g_{T}\left(E_{e}\right)}{1+3 \lambda^{2}}=-\frac{\alpha}{\pi} g_{F}\left(E_{e}\right),
$$

induced by one-virtual photon exchanges. Summing up the contributions of $\delta^{(j)} \mathcal{M}_{p e}$ with $j=1,2,3$ we obtain the following expression for $\delta \mathcal{M}_{p e}^{(\gamma)}$

$$
\begin{aligned}
& \delta \mathcal{M}_{p e}^{(\gamma)}=\delta^{(1)} \mathcal{M}_{p e}^{(\gamma)}+\delta^{(2)} \mathcal{M}_{p e}^{(\gamma)}+\delta^{(3)} \mathcal{M}_{p e}^{(\gamma)}=2 m_{n} \frac{\alpha}{2 \pi}\left\{(\lambda+1)\left[-\frac{3}{2} \ln \left(\frac{\Lambda}{m_{p}}\right)-\frac{3}{4}\right]+\frac{1}{2 \beta} \ln \left(\frac{1+\beta}{1-\beta}\right)\right\} \\
& \times\left[\varphi_{p}^{\dagger} \varphi_{n}\right]\left[\bar{u}_{e} \gamma^{0}\left(1-\gamma^{5}\right) v_{\bar{\nu}}\right]+2 m_{n} \frac{\alpha}{2 \pi}\left\{(\lambda+1)\left[+\frac{3}{2} \ln \left(\frac{\Lambda}{m_{p}}\right)+\frac{1}{4}\right]-\lambda \frac{1}{2 \beta} \ln \left(\frac{1+\beta}{1-\beta}\right)\right\}\left[\varphi_{p}^{\dagger} \vec{\sigma} \varphi_{n}\right] \cdot\left[\bar{u}_{e} \vec{\gamma}\left(1-\gamma^{5}\right) v_{\bar{\nu}}\right] \\
& -2 m_{n} \frac{\alpha}{2 \pi} g_{F}\left(E_{e}\right)\left\{\left[\varphi_{p}^{\dagger} \varphi_{n}\right]\left[\bar{u}_{e}\left(1-\gamma^{5}\right) v_{\bar{\nu}}\right]-\lambda\left[\varphi_{p}^{\dagger} \vec{\sigma} \varphi_{n}\right] \cdot\left[\bar{u}_{e} \gamma^{0} \vec{\gamma}\left(1-\gamma^{5}\right) v_{\bar{\nu}}\right]\right\}
\end{aligned}
$$

Summing up the contributions of Eq. (D-27) and Eq. (D-46) we calculate $\mathcal{M}_{p p}^{(\gamma)}+\mathcal{M}_{e e}^{(\gamma)}+\mathcal{M}_{p e}^{(\gamma)}$. Using this expression we get the amplitude of the continuum-state $\beta^{-}$-decay of the neutron, calculated in the non-relativistic approximation for the proton and taking into account the contributions of one--virtual photon exchanges. We represent it in the form

$$
\begin{aligned}
& M\left(n \rightarrow p e^{-} \bar{\nu}_{e}\right)=-2 m_{n} \frac{G_{F}^{(r)}}{\sqrt{2}} V_{u d}\left\{\left(1+\frac{\alpha}{2 \pi} f_{\beta_{c}^{-}}\left(E_{e}, \mu\right)\right)\left[\varphi_{p}^{\dagger} \varphi_{n}\right]\left[\bar{u}_{e} \gamma^{0}\left(1-\gamma^{5}\right) v_{\bar{\nu}}\right]\right. \\
& -\lambda^{(r)}\left(1+\frac{\alpha}{2 \pi} f_{\beta_{c}^{-}}\left(E_{e}, \mu\right)\right)\left[\varphi_{p}^{\dagger} \vec{\sigma} \varphi_{n}\right] \cdot\left[\bar{u}_{e} \vec{\gamma}\left(1-\gamma^{5}\right) v_{\bar{\nu}}\right]-\frac{\alpha}{2 \pi} g_{F}\left(E_{e}\right)\left[\varphi_{p}^{\dagger} \varphi_{n}\right]\left[\bar{u}_{e}\left(1-\gamma^{5}\right) v_{\bar{\nu}}\right] \\
& \left.+\frac{\alpha}{2 \pi} \lambda^{(r)} g_{F}\left(E_{e}\right)\left[\varphi_{p}^{\dagger} \vec{\sigma} \varphi_{n}\right] \cdot\left[\bar{u}_{e} \gamma^{0} \vec{\gamma}\left(1-\gamma^{5}\right) v_{\bar{\nu}}\right]\right\} .
\end{aligned}
$$

Here $G_{F}^{(r)}$ and $g_{A}^{(r)}$ are the renormalized Fermi and axial coupling constants

$$
G_{F}^{(r)}=G_{F}\left(1+\frac{\alpha}{2 \pi} d_{V}\right), \lambda^{(r)}=\lambda\left(1+\frac{\alpha}{2 \pi} d_{A}\right)
$$


where $d_{V}$ and $d_{A}$ are ultra-violet divergent constants. In our calculation of the radiative corrections they are equal to

$$
\begin{aligned}
& d_{V}=3 \ln \left(\frac{\Lambda}{m_{p}}\right)-\frac{1}{4}-c_{S}-(\lambda+1)\left[\frac{3}{2} \ln \left(\frac{\Lambda}{m_{p}}\right)+\frac{3}{4}\right] \\
& d_{A}=-\frac{\lambda+1}{\lambda}\left[\frac{3}{2} \ln \left(\frac{\Lambda}{m_{p}}\right)+\frac{1}{4}\right]+(\lambda+1)\left[\frac{3}{2} \ln \left(\frac{\Lambda}{m_{p}}\right)+\frac{3}{4}\right] .
\end{aligned}
$$

The function $f_{\beta_{c}^{-}}\left(E_{e}, \mu\right)$, given by

$$
f_{\beta_{c}^{-}}\left(E_{e}, \mu\right)=\frac{3}{2} \ln \left(\frac{m_{p}}{m_{e}}\right)+c_{S}+2 \ln \left(\frac{\mu}{m_{e}}\right)\left[\frac{1}{2 \beta} \ln \left(\frac{1+\beta}{1-\beta}\right)-1\right]+\frac{1}{\beta} L\left(\frac{2 \beta}{1+\beta}\right)-\frac{1}{4 \beta} \ln ^{2}\left(\frac{1+\beta}{1-\beta}\right)+\frac{1}{2 \beta} \ln \left(\frac{1+\beta}{1-\beta}\right),
$$

contains two terms $(3 / 2) \ln \left(m_{p} / m_{e}\right)$ and $c_{S}$ independent of the electron energy $E_{e}$. As we show in Appendix E the term $(3 / 2) \ln \left(m_{p} / m_{e}\right)$ is fixed by the KLN theorem. In our calculation the constant $c_{S}$ reflects an ambiguous decomposition of the contribution of one-virtual photon exchanges to the amplitude of the neutron $\beta^{-}-$decay into the renormalisation constant $d_{V}$ of the Fermi coupling constant and the radiative corrections to the lifetime of the neutron. Nevertheless, following Sirlin [18] and Abers et al. [19] one can show that due to a requirement of gauge invariance of the observable radiative corrections the value of the constant $c_{S}$ is fixed and is equal to $c_{S}=-11 / 8$. We show this in Appendix F.

The contribution of the $W$-boson and $Z$-boson exchanges and the QCD corrections [28] we describe by the constant $C_{W Z}$ [32]. The numerical value $C_{W Z}=10.249$ we discuss below Eq.(D-58). As a result the function $f_{\beta_{c}^{-}}\left(E_{e}, \mu\right)$ is

$$
\begin{aligned}
& f_{\beta_{c}^{-}}\left(E_{e}, \mu\right)=\frac{3}{2} \ln \left(\frac{m_{p}}{m_{e}}\right)-\frac{11}{8}+2 \ln \left(\frac{\mu}{m_{e}}\right)\left[\frac{1}{2 \beta} \ln \left(\frac{1+\beta}{1-\beta}\right)-1\right]+\frac{1}{\beta} L\left(\frac{2 \beta}{1+\beta}\right)-\frac{1}{4 \beta} \ln ^{2}\left(\frac{1+\beta}{1-\beta}\right)+\frac{1}{2 \beta} \ln \left(\frac{1+\beta}{1-\beta}\right) \\
& +C_{W Z} .
\end{aligned}
$$

Making a replacement $G_{F}^{(r)} \rightarrow G_{F}$ and $\lambda^{(r)} \rightarrow \lambda$ and taking into account the contributions of the "weak magnetism" and the proton recoil the amplitude of the continuum-state $\beta^{-}$-decay of the neutron takes the form

$$
\begin{aligned}
& M\left(n \rightarrow p e^{-} \bar{\nu}_{e}\right)=-2 m_{n} \frac{G_{F}}{\sqrt{2}} V_{u d}\left\{\left(1+\frac{\alpha}{2 \pi} f_{\beta_{c}^{-}}\left(E_{e}, \mu\right)\right)\left[\varphi_{p}^{\dagger} \varphi_{n}\right]\left[\bar{u}_{e} \gamma^{0}\left(1-\gamma^{5}\right) v_{\bar{\nu}}\right]\right. \\
& -\tilde{\lambda}\left(1+\frac{\alpha}{2 \pi} f_{\beta_{c}^{-}}\left(E_{e}, \mu\right)\right)\left[\varphi_{p}^{\dagger} \vec{\sigma} \varphi_{n}\right] \cdot\left[\bar{u}_{e} \vec{\gamma}\left(1-\gamma^{5}\right) v_{\bar{\nu}}\right]-\frac{\alpha}{2 \pi} g_{F}\left(E_{e}\right)\left[\varphi_{p}^{\dagger} \varphi_{n}\right]\left[\bar{u}_{e}\left(1-\gamma^{5}\right) v_{\bar{\nu}}\right] \\
& +\frac{\alpha}{2 \pi} \tilde{\lambda} g_{F}\left(E_{e}\right)\left[\varphi_{p}^{\dagger} \vec{\sigma} \varphi_{n}\right] \cdot\left[\bar{u}_{e} \gamma^{0} \vec{\gamma}\left(1-\gamma^{5}\right) v_{\bar{\nu}}\right]-\frac{m_{e}}{2 M}\left[\varphi_{p}^{\dagger} \varphi_{n}\right]\left[\bar{u}_{e}\left(1-\gamma^{5}\right) v_{\bar{\nu}}\right] \\
& \left.+\frac{\tilde{\lambda}}{2 M}\left[\varphi_{p}^{\dagger}\left(\vec{\sigma} \cdot \vec{k}_{p}\right) \varphi_{n}\right]\left[\bar{u}_{e} \gamma^{0}\left(1-\gamma^{5}\right) v_{\bar{\nu}}\right]-i \frac{\kappa+1}{2 M}\left[\varphi_{p}^{\dagger}\left(\vec{\sigma} \times \vec{k}_{p}\right) \varphi_{n}\right] \cdot\left[\bar{u}_{e} \vec{\gamma}\left(1-\gamma^{5}\right) v_{\bar{\nu}}\right]\right\},
\end{aligned}
$$

where $\tilde{\lambda}=\lambda\left(1-E_{0} / 2 M\right)$ and $\vec{k}_{p}=-\vec{k}_{e}-\vec{k}$ is the proton 3 -momentum in the rest frame of the neutron. The amplitude Eq.(D-52) has been used for the calculation of the electron-energy and angular distribution Eq.(66).

The radiative corrections to the rate of the continuum-state $\beta^{-}$-decay of the neutron, which we denote as $g_{\beta_{c}^{-}}\left(E_{e}, \mu\right)$, acquire an additional contribution of the electromagnetic Fierz term

$$
\begin{aligned}
& g_{\beta_{c}^{-}}\left(E_{e}, \mu\right)=f_{\beta_{c}^{-}}\left(E_{e}, \mu\right)+\frac{\pi}{\alpha} b_{F}^{(\mathrm{em}]}\left(E_{e}\right) \frac{m_{e}}{E_{e}}=f_{\beta_{c}^{-}}\left(E_{e}, \mu\right)-g_{F}\left(E_{e}\right) \frac{m_{e}}{E_{e}}=\frac{3}{2} \ln \left(\frac{m_{p}}{m_{e}}\right)-\frac{11}{8} \\
& +2 \ln \left(\frac{\mu}{m_{e}}\right)\left[\frac{1}{2 \beta} \ln \left(\frac{1+\beta}{1-\beta}\right)-1\right]+\frac{1}{\beta} L\left(\frac{2 \beta}{1+\beta}\right)-\frac{1}{4 \beta} \ln ^{2}\left(\frac{1+\beta}{1-\beta}\right)+\frac{\beta}{2} \ln \left(\frac{1+\beta}{1-\beta}\right)+C_{W Z},
\end{aligned}
$$

where the term, proportional to $\beta / 2$, is defined by

$$
\frac{\beta}{2} \ln \left(\frac{1+\beta}{1-\beta}\right)=\frac{1}{2 \beta} \ln \left(\frac{1+\beta}{1-\beta}\right)-g_{F}\left(E_{e}\right) \frac{m_{e}}{E_{e}} .
$$

For the calculation of the contributions of the effective scalar and tensor interactions, induced by one-virtual photon exchanges, to the correlation coefficients of the electron-energy and angular distribution of the neutron $\beta^{-}-$decay we 
use the results obtained in [10] (see Eq.(28) of Ref.[10]). The corrections to the correlation coefficients from the scalar and tensor lepton-nucleon weak interactions with the left-handed neutrinos take the form

$$
\begin{aligned}
\delta \zeta\left(E_{e}\right) & =b_{F} \frac{m_{e}}{E_{e}}=2 \frac{g_{S}-3 \lambda g_{T}}{1+3 \lambda^{2}} \frac{m_{e}}{E_{e}} \\
\delta a\left(E_{e}\right) & =0 \\
\delta A\left(E_{e}\right) & =0 \\
\delta B\left(E_{e}\right) & =2 \frac{\left(g_{T}-\lambda g_{S}\right)-2 \lambda g_{T}}{1+3 \lambda^{2}} \frac{m_{e}}{E_{e}} .
\end{aligned}
$$

where we have kept only the linear terms in the scalar and tensor coupling constant expansions. Replacing the scalar and tensor coupling constants by their expressions, given in Eq.(D-44), we obtain

$$
\begin{aligned}
\delta \zeta\left(E_{e}\right) & =b_{F} \frac{m_{e}}{E_{e}}=2 \frac{g_{S}-3 \lambda g_{T}}{1+3 \lambda^{2}} \frac{m_{e}}{E_{e}}=-\frac{\alpha}{\pi} g_{F} \frac{m_{e}}{E_{e}}, \\
\delta a\left(E_{e}\right) & =0 \\
\delta A\left(E_{e}\right) & =0, \\
\delta B\left(E_{e}\right) & =2 \frac{\left(g_{T}-\lambda g_{S}\right)-2 \lambda g_{T}}{1+3 \lambda^{2}} \frac{m_{e}}{E_{e}}=-B_{0} \frac{\alpha}{\pi} g_{F} \frac{m_{e}}{E_{e}} .
\end{aligned}
$$

This gives the following radiative corrections to the correlation coefficients

$$
\begin{aligned}
& \zeta\left(E_{e}\right)=\left(1+\frac{\alpha}{\pi} g_{n}\left(E_{e}\right)\right)+O(1 / M), \\
& a\left(E_{e}\right)=a_{0}\left(1+\frac{\alpha}{\pi} f_{n}\left(E_{e}\right)\right)+O(1 / M), \\
& A\left(E_{e}\right)=A_{0}\left(1+\frac{\alpha}{\pi} f_{n}\left(E_{e}\right)\right)+O(1 / M), \\
& B\left(E_{e}\right)=B_{0}+O(1 / M),
\end{aligned}
$$

where the terms $-g_{F} m_{e} / E_{e}$ are absorbed by the function $g_{n}\left(E_{e}\right)$ (see Eq.(D-53) $)$. The terms of order of $O(1 / M)$ are adduced in Eqs.(9) - (13).

The functions $g_{n}\left(E_{e}\right)$ and $f_{n}\left(E_{e}\right)$ are defined by

$$
\begin{aligned}
& g_{n}\left(E_{e}\right)=\lim _{\mu \rightarrow 0}\left[g_{\beta_{c}^{-}}\left(E_{e}, \mu\right)+g_{\beta_{c}^{-} \gamma}^{(1)}\left(E_{e}, \mu\right)\right]=\frac{3}{2} \ln \left(\frac{m_{p}}{m_{e}}\right)-\frac{3}{8}+2\left[\frac{1}{2 \beta} \ln \left(\frac{1+\beta}{1-\beta}\right)-1\right]\left[\ln \left(\frac{2\left(E_{0}-E_{e}\right)}{m_{e}}\right)\right. \\
& \left.-\frac{3}{2}+\frac{1}{3} \frac{E_{0}-E_{e}}{E_{e}}\right]+\frac{2}{\beta} L\left(\frac{2 \beta}{1+\beta}\right)+\frac{1}{2 \beta} \ln \left(\frac{1+\beta}{1-\beta}\right)\left[\left(1+\beta^{2}\right)+\frac{1}{12} \frac{\left(E_{0}-E_{e}\right)^{2}}{E_{e}^{2}}-\ln \left(\frac{1+\beta}{1-\beta}\right)\right]+C_{W Z}, \\
& f_{n}\left(E_{e}\right)=\lim _{\mu \rightarrow 0}\left[g_{\beta_{c}^{-} \gamma}^{(2)}\left(E_{e}, \mu\right)-g_{\beta_{c}^{-} \gamma}^{(1)}\left(E_{e}, \mu\right)\right]+g_{F}\left(E_{e}\right) \frac{m_{e}}{E_{e}}=\frac{2}{3} \frac{E_{0}-E_{e}}{E_{e}}\left(1+\frac{1}{8} \frac{E_{0}-E_{e}}{E_{e}}\right) \frac{1-\beta^{2}}{\beta^{2}} \\
& \times\left[\frac{1}{2 \beta} \ln \left(\frac{1+\beta}{1-\beta}\right)-1\right]-\frac{1}{12} \frac{\left(E_{0}-E_{e}\right)^{2}}{E_{e}^{2}}+\frac{1-\beta^{2}}{2 \beta} \ln \left(\frac{1+\beta}{1-\beta}\right)
\end{aligned}
$$

where the functions $g_{\beta_{c}^{-} \gamma}^{(1)}\left(E_{e}, \mu\right)$ and $g_{\beta_{c}^{-} \gamma}^{(2)}\left(E_{e}, \mu\right)$ are given in Eq. (B-28).

The $g_{n}\left(E_{e}\right)$ and $f_{n}\left(E_{e}\right)$, multiplied by $\alpha / \pi$, are in analytical agreement with results, obtained in [18] -30] and [31, 32], respectively. The constant $C_{W Z}$, defined by the contributions of the $W$-boson and $Z$-boson exchanges and the QCD corrections [28], is equal to $C_{W Z}=10.249$. This numerical value is obtained from the fit of the radiative corrections to the lifetime of the neutron $(\alpha / \pi)\left\langle g_{n}\left(E_{e}\right)\right\rangle=0.03886(39)[1]$ and $(\alpha / \pi)\left\langle g_{n}\left(E_{e}\right)\right\rangle=0.0390(8)$ [28], averaged over the phase volume of the neutron decay.

The radiative corrections $(\alpha / \pi) g_{n}\left(E_{e}\right)$ and $(\alpha / \pi) f_{n}\left(E_{e}\right)$, weighted with the electron energy spectrum density

$$
\rho_{\beta_{c}^{-}}\left(E_{e}\right)=\left(E_{0}-E_{e}\right)^{2} \sqrt{E_{e}^{2}-m_{e}^{2}} E_{e} \zeta\left(E_{e}\right) \frac{F\left(E_{e}, Z=1\right)}{f_{n}\left(E_{0}, Z=1\right)},
$$

where the functions $\zeta\left(E_{e}\right)$ and $F\left(E_{e}, Z=1\right)$ are given in Eq. (17) and Eq.(15), respectively, and $f_{n}\left(E_{0}, Z=1\right)$ is the Fermi integral Eq.41], are plotted in Fig. 1. 
Finally we would like to note that having attributed the terms, proportional to $(\lambda+1)$ to the renormalisation constants of the Fermi and axial coupling constants only, we arrive at the radiative corrections, described by the function

$$
\begin{aligned}
g\left(E_{e}\right) & =g_{n}\left(E_{e}\right)+3 \ln \left(\frac{\Lambda}{m_{p}}\right)+\frac{9}{8}-C_{W Z}= \\
& =3 \ln \left(\frac{\Lambda}{m_{p}}\right)+\frac{3}{2} \ln \left(\frac{m_{p}}{m_{e}}\right)+\frac{3}{4}+2\left[\frac{1}{2 \beta} \ln \left(\frac{1+\beta}{1-\beta}\right)-1\right]\left[\ln \left(\frac{2\left(E_{0}-E_{e}\right)}{m_{e}}\right)-\frac{3}{2}+\frac{1}{3} \frac{E_{0}-E_{e}}{E_{e}}\right] \\
& +\frac{2}{\beta} L\left(\frac{2 \beta}{1+\beta}\right)+\frac{1}{2 \beta} \ln \left(\frac{1+\beta}{1-\beta}\right)\left[\left(1+\beta^{2}\right)+\frac{1}{12} \frac{\left(E_{0}-E_{e}\right)^{2}}{E_{e}^{2}}-\ln \left(\frac{1+\beta}{1-\beta}\right)\right]
\end{aligned}
$$

The function $g\left(E_{e}\right)$, multiplied by $\alpha / \pi$, agrees analytically with the result, calculated by Kinoshita and Sirlin 15$]$.

\section{Appendix E: Kinoshita-Lee-Nauenberg theorem for radiative corrections of neutron $\beta^{-}-$decay}

As has been shown by Kinoshita and Sirlin [15], the radiative corrections to the rates of the muon decay $\mu^{-} \rightarrow$ $e^{-}+\nu_{\mu}+\bar{\nu}_{e}$ and of the neutron $\beta^{-}$-decay, taken in the limit $m_{e} \rightarrow 0$ and integrated over the phase volume, does not depend on the electron mass. This is so-called the Kinoshita-Lee-Nauenberg (KLN) theorem 36]. Thus, the radiative corrections to the rate of the neutron decay, described by the function $g_{n}\left(E_{e}\right)$, should obey the KLN theorem. This means that the result of the integration of the function $g_{n}\left(E_{e}\right)$, taken in the limit $m_{e} \rightarrow 0$, over the phase volume should not depend on $m_{e}$.

Sirlin's function $\bar{g}\left(E_{e}\right)=g_{n}\left(E_{e}\right)-C_{W Z}$, defining the radiative corrections, caused by one-virtual photon exchanges, to the lifetime of the neutron, takes the form [18]

$$
\begin{aligned}
& \bar{g}\left(E_{e}\right)=g_{n}\left(E_{e}\right)-C_{W Z}=\frac{3}{2} \ln \left(\frac{m_{p}}{m_{e}}\right)-\frac{3}{8}+2\left[\frac{1}{2 \beta} \ln \left(\frac{1+\beta}{1-\beta}\right)-1\right]\left[\ln \left(\frac{2\left(E_{0}-E_{e}\right)}{m_{e}}\right)-\frac{3}{2}+\frac{1}{3} \frac{E_{0}-E_{e}}{E_{e}}\right] \\
& +\frac{2}{\beta} L\left(\frac{2 \beta}{1+\beta}\right)+\frac{1}{2 \beta} \ln \left(\frac{1+\beta}{1-\beta}\right)\left[\left(1+\beta^{2}\right)+\frac{1}{12} \frac{\left(E_{0}-E_{e}\right)^{2}}{E_{e}^{2}}-\ln \left(\frac{1+\beta}{1-\beta}\right)\right] .
\end{aligned}
$$

Taking the limit $E_{e} \gg m_{e}$, corresponding to $\beta \rightarrow 1$, and introducing the variable $x=E_{e} / E_{0}$ we transcribe the function $g\left(E_{e}\right)$ into the form

$$
\begin{aligned}
& \bar{g}\left(E_{e}\right) \rightarrow \bar{g}\left(x, m_{e}\right)=\frac{3}{2} \ln \left(\frac{m_{p}}{2 E_{0}}\right)+\frac{3}{2} \ln \left(\frac{2 E_{0}}{m_{e}}\right)-\frac{3}{8}+2\left(\ln x+\ln \left(\frac{2 E_{0}}{m_{e}}\right)-1\right)\left[\ln \left(\frac{1-x}{x}\right)-\frac{3}{2}+\frac{1}{3} \frac{1-x}{x}\right. \\
& \left.+\left(\ln x+\ln \left(\frac{2 E_{0}}{m_{e}}\right)\right)\right]+2 L(1)+\left(\ln x+\ln \left(\frac{2 E_{0}}{m_{e}}\right)\right)\left[2+\frac{1}{12} \frac{(1-x)^{2}}{x^{2}}-2\left(\ln x+\ln \left(\frac{2 E_{0}}{m_{e}}\right)\right)\right] .
\end{aligned}
$$

Thus, the function $\bar{g}\left(x, m_{e}\right)$ is equal to

$$
\begin{aligned}
& \bar{g}\left(x, m_{e}\right)=\frac{3}{2} \ln \left(\frac{m_{p}}{2 E_{0}}\right)-\frac{3}{8}-\frac{\pi^{2}}{3}+2(\ln x-1)\left[\ln \left(\frac{1-x}{x}\right)-\frac{3}{2}+\frac{1}{3} \frac{1-x}{x}\right] \\
& +\frac{1}{12} \frac{(1-x)^{2}}{x^{2}} \ln x+\ln \left(\frac{2 E_{0}}{m_{e}}\right)\left[2 \ln \left(\frac{1-x}{x}\right)-\frac{3}{2}+\frac{2}{3} \frac{1-x}{x}+\frac{1}{12} \frac{(1-x)^{2}}{x^{2}}\right] .
\end{aligned}
$$

For the derivation Eq. E-3) we have used $L(1)=-\pi^{2} / 6$ and the approximation

$$
\ln \left(\frac{1+\beta}{1-\beta}\right) \rightarrow 2 \ln x+2 \ln \left(\frac{2 E_{0}}{m_{e}}\right) .
$$

According to the KLN theorem [36] (see also [15]), the function $\bar{g}\left(x, m_{e}\right)$, integrated over the phase volume, taken in the limit $m_{e} \rightarrow 0$, should not depend on $m_{e}$. We would like to emphasize that the quadratic terms $\ell n^{2}\left(2 E_{0} / m_{e}\right)$ are cancelled in the function $\bar{g}\left(x, m_{e}\right)$ without integration over the phase volume.

Now let check the contribution of the linear terms $\ln \left(2 E_{0} / m_{e}\right)$. For this aim we have to integrate the function $g\left(x, m_{e}\right)$ over the phase volume. The integration of the function $g\left(x, m_{e}\right)$ over the phase volume with a dimensionless element $(1-x)^{2} x^{2} d x$, obtained at $m_{e} \rightarrow 0$, gives the result independent of $m_{e}$, since

$$
\int_{0}^{1}\left[2 \ln \left(\frac{1-x}{x}\right)-\frac{3}{2}+\frac{2}{3} \frac{1-x}{x}+\frac{1}{12} \frac{(1-x)^{2}}{x^{2}}\right](1-x)^{2} x^{2} d x=0 .
$$


Thus, the term $\ln \left(2 E_{0} / m_{e}\right)$ vanishes. This reproduces the results, obtained in [15], and confirms the KLN theorem [36].

We would like to note that the term $-3 / 2$ in the integrand of Eq. (E-5), playing an important role for the vanishing of the integral, is given by $-3 / 2=-3+3 / 2$, where -3 and $+3 / 2$ come from the energy depending part of the function $\bar{g}\left(E_{e}\right)$ and the term $(3 / 2) \ell n\left(m_{p} / m_{e}\right)$, respectively.

\section{Appendix F: Comparison with Sirlin's calculation of radiative corrections [18]}

In this Appendix we compare our calculation of the radiative corrections to the continuum-state $\beta^{-}-$decay of the neutron, caused by one-virtual photon exchanges, with the calculation, carried out by Sirlin in his well-known paper [18].

According to Sirlin [18], the radiative corrections to the amplitude of the continuum-state $\beta^{-}$-decay of the neutron are defined by Eqs.(9a), (11), (14) and (19) of Ref.[18]. Now let us compare our expressions with Sirlin's ones.

Sirlin's Eq.(9a) corresponds to our amplitude $\mathcal{M}_{p e}$. In order to show this we rewrite the amplitude $\mathcal{M}_{p e}$, given by Eq. (D-2), as follows

$$
\mathcal{M}_{p e}^{(\gamma)}=-\frac{\alpha}{4 \pi} \int \frac{d^{4} q}{\pi^{2} i} D_{\alpha \beta}(q)\left[\bar{u}_{e} \gamma^{\alpha} \frac{1}{m_{e}-\hat{k}_{e}-\hat{q}-i 0} O_{\mu} v_{\bar{\nu}}\right]\left[\bar{u}_{p} \gamma^{\beta} \frac{1}{m_{p}-\hat{k}_{p}+\hat{q}-i 0} W^{\mu} u_{n}\right],
$$

where $D_{\alpha \beta}(q)$ is the photon Green function

$$
D_{\alpha \beta}(q)=\frac{1}{q^{2}+i 0}\left(g_{\alpha \beta}-\xi \frac{q_{\alpha} q_{\beta}}{q^{2}+i 0}\right)
$$

in the arbitrary gauge with a gauge parameter $\xi, W^{\mu}$ and $O_{\mu}$ are the products of the Dirac matrices defined by

$$
W^{\mu}=\gamma^{\mu}\left(1+\lambda \gamma^{5}\right) \quad, \quad O_{\mu}=\gamma_{\mu}\left(1-\gamma^{5}\right)
$$

After some algebraical transformations Eq. F-1) can be reduced to Sirlin's form

$$
\mathcal{M}_{p e}^{(\gamma)}=-\frac{\alpha}{4 \pi} \int \frac{d^{4} q}{\pi^{2} i} D_{\alpha \beta}(q) \frac{\left[\bar{u}_{e}\left(2 k_{e}^{\alpha}+\gamma^{\alpha} \hat{q}\right) O_{\mu} v_{\bar{\nu}}\right]}{q^{2}+2 k_{e} \cdot q+i 0} \bar{u}_{p}\left[\frac{\left(2 k_{p}^{\beta}-q^{\beta}\right) W^{\mu}}{q^{2}-2 k_{p} \cdot q+i 0}+T^{\beta \mu}\right] u_{n},
$$

where $T^{\beta \mu}$ is given by

$$
T^{\beta \mu}=\frac{R^{\beta \mu}}{q^{2}-2 k_{p} \cdot q+i 0}
$$

and $R^{\beta \mu}$ is equal to $R^{\beta \mu}=i \sigma^{\beta \lambda} q_{\lambda} W^{\mu}$. As has been pointed out by Sirlin [18], the tensor $T^{\beta \mu}$ is obviously transverse. $q_{\beta} T^{\beta \mu}=0$. Then, we propose to rewrite the amplitude Eq. (F-4) as follows

$$
\mathcal{M}_{p e}^{(\gamma)}=\mathcal{M}_{p e}^{(\mathrm{SC})}+\delta \mathcal{M}_{p e}^{(\mathrm{SLI})}
$$

where the indices (SC) and (SLI) mean "Sirlin's Corrections" and "Strong low-energy interactions". The amplitudes $\mathcal{M}_{p e}^{(\mathrm{SC})}$ and $\delta \mathcal{M}_{p e}^{(\mathrm{SLI})}$ are equal to

$$
\begin{aligned}
& \mathcal{M}_{p e}^{(\mathrm{SC})}=-\frac{\alpha}{4 \pi} \int \frac{d^{4} q}{\pi^{2} i} D_{\alpha \beta}(q) \frac{\left[\bar{u}_{e}\left(2 k_{e}^{\alpha}+\gamma^{\alpha} \hat{q}\right) O_{\mu} v_{\bar{\nu}}\right]}{q^{2}+2 k_{e} \cdot q+i 0} \frac{\left[\bar{u}_{p}\left(2 k_{p}^{\beta}-q^{\beta}\right) W^{\mu} u_{n}\right]}{q^{2}-2 k_{p} \cdot q+i 0} \\
& \delta \mathcal{M}_{p e}^{(\mathrm{SLI})}=-\frac{\alpha}{4 \pi} \int \frac{d^{4} q}{\pi^{2} i} D_{\alpha \beta}(q) \frac{\left[\bar{u}_{e}\left(2 k_{e}^{\alpha}+\gamma^{\alpha} \hat{q}\right) O_{\mu} v_{\bar{\nu}}\right]}{q^{2}+2 k_{e} \cdot q+i 0}\left[\bar{u}_{p} T^{\beta \mu} u_{n}\right] .
\end{aligned}
$$

The contribution of Sirlin's Eq.(11) coincides with the part of the amplitude $\mathcal{M}_{e e}^{(\gamma)}$, defining renormalisation of the wave function of the electron by electromagnetic interactions. The amplitude $\mathcal{M}_{e e}^{(\gamma)}$, given by Eq. (D-2), we rewrite as

$$
\mathcal{M}_{e e}^{(\gamma)}=\mathcal{M}_{e e}^{(\mathrm{SC})}+\delta \mathcal{M}_{e e}^{(\mathrm{SLI})}
$$


where the amplitudes $\mathcal{M}_{e e}^{(\mathrm{SC})}$ and $\delta \mathcal{M}_{e e}^{(\mathrm{SLI})}$ are equal to

$$
\begin{aligned}
& \mathcal{M}_{e e}^{(\mathrm{SC})}=-\frac{\alpha}{8 \pi m_{e}}\left[\bar{u}_{p} W^{\mu} u_{n}\right] \int \frac{d^{4} q}{\pi^{2} i} D_{\alpha \beta}(q) \frac{\left[\bar{u}_{e}\left(2 k_{e}^{\alpha}+\gamma^{\alpha} \hat{q}\right) \hat{k}_{e}\left(2 k_{e}^{\beta}+\hat{q} \gamma^{\beta}\right) O_{\mu} v_{\bar{\nu}}\right]}{\left(q^{2}+2 k_{e} \cdot q+i 0\right)^{2}} \\
& \delta \mathcal{M}_{e e}^{(\mathrm{SLI})}=\frac{\alpha}{4 \pi}\left[\bar{u}_{p} W^{\mu} u_{n}\right] \int \frac{d^{4} q}{\pi^{2} i} D_{\alpha \beta}(q)\left[\bar{u}_{e} \gamma^{\alpha} \frac{1}{m_{e}-\hat{k}_{e}-\hat{q}-i 0} \gamma^{\beta} \frac{1}{m_{e}-\hat{k}_{e}-i 0} O_{\mu} v_{\bar{\nu}}\right] \\
& +\left[\bar{u}_{p} W^{\mu} u_{n}\right]\left[\bar{u}_{e}\left(-\delta m_{e}+\frac{Z_{2}^{(e)}-1}{2}\left(m_{e}-\hat{k}_{e}\right)\right) \frac{1}{m_{e}-\hat{k}_{e}-i 0} O_{\mu} v_{\bar{\nu}}\right] \\
& +\frac{\alpha}{8 \pi m_{e}}\left[\bar{u}_{p} W^{\mu} u_{n}\right] \int \frac{d^{4} q}{\pi^{2} i} D_{\alpha \beta}(q) \frac{\left[\bar{u}_{e}\left(2 k_{e}^{\alpha}+\gamma^{\alpha} \hat{q}\right) \hat{k}_{e}\left(2 k_{e}^{\beta}+\hat{q} \gamma^{\beta}\right) O_{\mu} v_{\bar{\nu}}\right]}{\left(q^{2}+2 k_{e} \cdot q+i 0\right)^{2}},
\end{aligned}
$$

respectively. For the derivation of Sirlin's term $\mathcal{M}_{e e}^{(\mathrm{SC})}$ one has to use the definition of the renormalisation constant $Z_{2}^{(e)}-1$ of the electron wave function [19, 35.

$\frac{Z_{2}^{(e)}-1}{2}=-\left.\frac{\alpha}{8 \pi} \frac{k_{e}^{\lambda}}{m_{e}} \int \frac{d^{4} q}{\pi^{2} i} D_{\alpha \beta}(q) \frac{\partial}{\partial k^{\lambda}} \gamma^{\alpha} \frac{1}{m_{e}-\hat{k}_{e}-\hat{q}} \gamma^{\beta}\right|_{\hat{k}_{e}=m_{e}}=-\left.\frac{\alpha}{8 \pi} \frac{k_{e}^{\lambda}}{m_{e}} \int \frac{d^{4} q}{\pi^{2} i} D_{\alpha \beta}(q) \frac{\partial}{\partial q^{\lambda}} \gamma^{\alpha} \frac{1}{m_{e}-\hat{k}_{e}-\hat{q}} \gamma^{\beta}\right|_{\hat{k}_{e}=m_{e}}=$

$=-\left.\frac{\alpha}{8 \pi m_{e}} \int \frac{d^{4} q}{\pi^{2} i} D_{\alpha \beta}(q) \frac{\left(2 k_{e}^{\alpha}+\gamma^{\alpha} \hat{q}\right) \hat{k}_{e}\left(2 k_{e}^{\beta}+\hat{q} \gamma^{\beta}\right)}{\left(q^{2}+2 k_{e} \cdot q+i 0\right)^{2}}\right|_{\hat{k}_{e}=m_{e}}$.

In comparison with Sirlin's expression, we have taken away the operator $\left(\hat{k}_{e}+m_{e}\right) / 2 m_{e}$, which is equal to unity at $\hat{k}_{e}=m_{e}$.

As has been pointed out by Sirlin, Eq.(14) of Ref. [18] is related to the emission and absorption of a photon by the proton. This means that Eq.(14) of Ref.[18] should be a part of the amplitude $\mathcal{M}_{p p}^{(\gamma)}$. This allows us to represent the amplitude $\mathcal{M}_{p p}^{(\gamma)}$ in the form

$$
\mathcal{M}_{p p}^{(\gamma)}=\mathcal{M}_{p p}^{(\mathrm{SC})}+\delta \mathcal{M}_{p p}^{(\mathrm{SLI})}
$$

with $\mathcal{M}_{p p}^{(\mathrm{SC})}$ and $\delta \mathcal{M}_{p p}^{(\mathrm{SLI})}$, given by

$$
\begin{aligned}
& \mathcal{M}_{p p}^{(\mathrm{SC})}=-\frac{\alpha}{8 \pi}\left[\bar{u}_{p} W^{\mu} u_{n}\right]\left[\bar{u}_{e} O_{\mu} v_{\bar{\nu}}\right] \int \frac{d^{4} q}{\pi^{2} i} D_{\alpha \beta}(q) \frac{\left(2 k_{p}-q\right)^{\alpha}\left(2 k_{p}-q\right)^{\beta}}{\left(q^{2}-2 k_{p} \cdot q+i 0\right)^{2}} \\
& \delta \mathcal{M}_{p p}^{(\mathrm{SLI})}=\frac{\alpha}{4 \pi} \int \frac{d^{4} q}{\pi^{2} i} D_{\alpha \beta}(q)\left[\bar{u}_{p} \gamma^{\alpha} \frac{1}{m_{p}-\hat{k}_{p}+\hat{q}-i 0} \gamma^{\beta} \frac{1}{m_{p}-\hat{k}_{p}-i 0} W^{\mu} u_{n}\right]\left[\bar{u}_{e} O_{\mu} v_{\bar{\nu}}\right] \\
& +\left[\bar{u}_{p}\left(-\delta m_{p}+\frac{Z_{2}^{(p)}-1}{2}\left(m_{p}-\hat{k}_{p}\right)\right) \frac{1}{m_{p}-\hat{k}_{p}-i 0} W^{\mu} u_{n}\right]\left[\bar{u}_{e} O_{\mu} v_{\bar{\nu}}\right] \\
& +\frac{\alpha}{8 \pi}\left[\bar{u}_{p} W^{\mu} u_{n}\right]\left[\bar{u}_{e} O_{\mu} v_{\bar{\nu}}\right] \int \frac{d^{4} q}{\pi^{2} i} D_{\alpha \beta}(q) \frac{\left(2 k_{p}-q\right)^{\alpha}\left(2 k_{p}-q\right)^{\beta}}{\left(q^{2}-2 k_{p} \cdot q+i 0\right)^{2}}
\end{aligned}
$$

respectively. As a result, according to Sirlin [18], the observable radiative corrections to the amplitude of the continuum-state $\beta^{-}$-decay of the neutron, caused by one--virtual photon exchange, are defined by the amplitude

$$
\mathcal{M}_{\mathrm{RC}}^{(\mathrm{SC})}=\mathcal{M}_{p e}^{(\mathrm{SC})}+\mathcal{M}_{e e}^{(\mathrm{SC})}+\mathcal{M}_{p p}^{(\mathrm{SC})}
$$

which is gauge invariant, i.e. invariant under the gauge transformation of the photon Green function $D_{\alpha \beta}(q) \rightarrow$ $D_{\alpha \beta}(q)+c\left(q^{2}\right) q_{\alpha} q_{\beta}$ with an arbitrary function $c\left(q^{2}\right)$, and suffers from the infrared divergences only [18]. The subscript RC means "Radiative Corrections". As we show below the additional contribution, described by the amplitude

$$
\delta \mathcal{M}_{\mathrm{RC}}^{(\mathrm{SLI})}=\delta \mathcal{M}_{p e}^{(\mathrm{SLI})}+\delta \mathcal{M}_{e e}^{(\mathrm{SLI})}+\delta \mathcal{M}_{p p}^{(\mathrm{SLI})}
$$

does not depend on the electron energy and should be absorbed by renormalisation of the Fermi coupling constant $G_{F}$ and axial coupling constant $\lambda$.

Since the amplitude $\mathcal{M}_{\mathrm{RC}}^{(\mathrm{SC})}$ is invariant under gauge transformations of the photon Green function $D_{\alpha \beta}(q) \rightarrow$ $D_{\alpha \beta}(q)+c\left(q^{2}\right) q_{\alpha} q_{\beta}$, we may calculate it by using the Feynman gauge for the photon Green function. The calculation 
of the amplitude $\delta \mathcal{M}_{\mathrm{RC}}^{(\mathrm{SLI})}$, describing the contributions to renormalisation constants of the Fermi and axial coupling constants, can be also carried out in the Feynman gauge.

The amplitude $\mathcal{M}_{p e}^{(\mathrm{SC})}$ can be rewritten as follows

$$
\begin{aligned}
& \mathcal{M}_{p e}^{(\mathrm{SC})}=\frac{\alpha}{4 \pi}\left[\bar{u}_{p} W^{\mu} u_{n}\right]\left\{[ \overline { u } _ { e } O _ { \mu } v _ { \overline { \nu } } ] \left[\int \frac{d^{4} q}{\pi^{2} i} \frac{1}{q^{2}+i 0} \frac{1}{q^{2}-2 k_{p} \cdot q+i 0}+\int \frac{d^{4} q}{\pi^{2} i} \frac{1}{q^{2}+i 0} \frac{1}{q^{2}+2 k_{e} \cdot q+i 0}\right.\right. \\
& \left.-\int \frac{d^{4} q}{\pi^{2} i} \frac{1}{q^{2}-2 k_{p} \cdot q+i 0} \frac{1}{q^{2}+2 k_{e} \cdot q+i 0}-4\left(k_{e} \cdot k_{p}\right) \int \frac{d^{4} q}{\pi^{2} i} \frac{1}{q^{2}+i 0} \frac{1}{q^{2}-2 k_{p} \cdot q+i 0} \frac{1}{q^{2}+2 k_{e} \cdot q+i 0}\right] \\
& \left.-2 i \int \frac{d^{4} q}{\pi^{2} i} \frac{1}{q^{2}+i 0} \frac{1}{q^{2}-2 k_{p} \cdot q+i 0} \frac{1}{q^{2}+2 k_{e} \cdot q+i 0}\left[\bar{u}_{e} \sigma_{\alpha \beta} q^{\alpha} k_{p}^{\beta} O_{\mu} v_{\bar{\nu}}\right]\right\} .
\end{aligned}
$$

All momentum integrals in Eq. ultra-violet divergent integrals and the FPM regularization for the infrared divergent contributions. Using the results, obtained in Appendix C and Appendix D (see Eqs.(C-15), (D-12) and (D-40), the amplitude $\mathcal{M}_{p e}^{(\mathrm{SC})}$ takes the form

$$
\begin{aligned}
& \mathcal{M}_{p e}^{(\mathrm{SC})}=\left[\bar{u}_{p} W^{\mu} u_{n}\right] \frac{\alpha}{2 \pi}\left\{[ \overline { u } _ { e } O _ { \mu } v _ { \overline { \nu } } ] \left[\ln \left(\frac{\Lambda}{m_{e}}\right)+\frac{1}{2}+\ln \left(\frac{\mu}{m_{e}}\right) \frac{1}{\beta} \ln \left(\frac{1+\beta}{1-\beta}\right)+\frac{1}{\beta} L\left(\frac{2 \beta}{1+\beta}\right)\right.\right. \\
& \left.\left.-\frac{1}{4 \beta} \ln ^{2}\left(\frac{1+\beta}{1-\beta}\right)+\frac{1}{2 \beta} \ln \left(\frac{1+\beta}{1-\beta}\right)\right]-\left[\bar{u}_{e} \gamma^{0} O_{\mu} v_{\bar{\nu}}\right] \frac{\sqrt{1-\beta^{2}}}{2 \beta} \ln \left(\frac{1+\beta}{1-\beta}\right)\right\} .
\end{aligned}
$$

The amplitude $\mathcal{M}_{e e}^{(\mathrm{SC})}$ can be transcribed into the form

$$
\begin{aligned}
& \mathcal{M}_{e e}^{(\mathrm{SC})}=-\frac{\alpha}{8 \pi m_{e}}\left[\bar{u}_{p} W^{\mu} u_{n}\right] \int \frac{d^{4} q}{\pi^{2} i} D_{\alpha \beta}(q) \frac{\left[\bar{u}_{e}\left(2 k_{e}^{\alpha}+\gamma^{\alpha} \hat{q}\right) \hat{k}_{e}\left(2 k_{e}^{\beta}+\hat{q} \gamma^{\beta}\right) O_{\mu} v_{\bar{\nu}}\right]}{\left(q^{2}+2 k_{e} \cdot q+i 0\right)^{2}} \\
& =-\frac{\alpha}{4 \pi m_{e}}\left[\bar{u}_{p} W^{\mu} u_{n}\right] \int \frac{d^{4} q}{\pi^{2} i} \frac{1}{q^{2}+i 0} \frac{\left[\bar{u}_{e}\left(2 m_{e}^{3}+2 m_{e} \hat{q} \hat{k}_{e}-\hat{q} \hat{k}_{e} \hat{q}\right) O_{\mu} v_{\bar{\nu}}\right]}{\left(q^{2}+2 k_{e} \cdot q+i 0\right)^{2}} \rightarrow-\frac{\alpha}{4 \pi m_{e}}\left[\bar{u}_{p} W^{\mu} u_{n}\right] \\
& \times \int_{0}^{1} d x 2 x \int \frac{d^{4} q}{\pi^{2} i}\left\{\frac{\left[\bar{u}_{e}\left(2 m_{e}^{3}+2 m_{e} \hat{q} \hat{k}_{e}-\hat{q} \hat{k}_{e} \hat{q}\right) O_{\mu} v_{\bar{\nu}}\right]}{\left(\left(q+k_{e} x\right)^{2}-m_{e}^{2} x^{2}-\mu^{2}(1-x)\right)^{3}}-\frac{\left[\bar{u}_{e}\left(2 m_{e}^{3}+2 m_{e} \hat{q} \hat{k}_{e}-\hat{q} \hat{k}_{e} \hat{q}\right) O_{\mu} v_{\bar{\nu}}\right]}{\left(\left(q+k_{e} x\right)^{2}-m_{e}^{2} x^{2}-\Lambda^{2}(1-x)\right)^{3}}=\right. \\
& =\frac{\alpha}{8 \pi}\left[\bar{u}_{p} W^{\mu} u_{n}\right]\left[\bar{u}_{e} O_{\mu} v_{\bar{\nu}}\right] \int_{0}^{1} d x 2 x \int \frac{d^{4} q}{\pi^{2}}\left[\frac{4 m_{e}^{2}\left(1-x-x^{2}\right)-q^{2}}{\left(q^{2}+m_{e}^{2} x^{2}+\mu^{2}(1-x)\right)^{3}}-\frac{m_{e}^{2}\left(4-4 x-2 x^{2}\right)-q^{2}}{\left(q^{2}+m_{e}^{2} x^{2}+\Lambda^{2}(1-x)\right)^{3}}\right]= \\
& =\frac{\alpha}{8 \pi}\left[\bar{u}_{p} W^{\mu} u_{n}\right]\left[\bar{u}_{e} O_{\mu} v_{\bar{\nu}}\right] \int_{0}^{1} d x 2 x \int \frac{d^{4} q}{\pi^{2}}\left[\frac{m_{e}^{2}\left(4-4 x-x^{2}\right)}{\left(q^{2}+m_{e}^{2} x^{2}+\mu^{2}(1-x)\right)^{3}}-\frac{m_{e}^{2}\left(4-4 x-x^{2}\right)+\Lambda^{2}(1-x)}{\left(q^{2}+m_{e}^{2} x^{2}+\Lambda^{2}(1-x)\right)^{3}}\right. \\
& \times-\frac{\alpha}{16 \pi}\left[\bar{u}_{p} W^{\mu} u_{n}\right]\left[\bar{u}_{e} O_{\mu} v_{\bar{\nu}}\right] \\
& \times \int_{0}^{1} d x 2 x\left\{\frac{m_{e}^{2}\left(4-4 x-x^{2}\right)}{m_{e}^{2} x^{2}+\mu^{2}(1-x)}-\frac{m_{e}^{2}\left(4-4 x-x^{2}\right)+\Lambda^{2}(1-x)}{m_{e}^{2} x^{2}+\mu^{2}+\Lambda^{2}(1-x)}-2 \ell n\left[\frac{\Lambda^{2}(1-x)}{m_{e}^{2} x^{2}}\right]\right\} .
\end{aligned}
$$

Having integrated over $x$ we arrive at the following expression for the amplitude $\mathcal{M}_{e e}^{(\mathrm{SC})}$

$$
\mathcal{M}_{e e}^{(\mathrm{SC})}=\left[\bar{u}_{p} W^{\mu} u_{n}\right]\left[\bar{u}_{e} O_{\mu} v_{\bar{\nu}}\right] \frac{\alpha}{2 \pi}\left[-\frac{1}{2} \ln \left(\frac{\Lambda}{m_{e}}\right)-\ln \left(\frac{\mu}{m_{e}}\right)-\frac{9}{8}\right] .
$$

In the Feynman gauge the amplitude $\mathcal{M}_{p p}^{(\mathrm{SC})}$ takes the form

$$
\begin{aligned}
& \mathcal{M}_{p p}^{(\mathrm{SC})}=-\frac{\alpha}{8 \pi}\left[\bar{u}_{p} W^{\mu} u_{n}\right]\left[\bar{u}_{e} O_{\mu} v_{\bar{\nu}}\right] \int \frac{d^{4} q}{\pi^{2} i} \frac{1}{q^{2}+i 0} \frac{4 m_{p}^{2}-4\left(k_{p} \cdot q\right)+q^{2}}{\left(q^{2}-2 k_{p} \cdot q+i 0\right)^{2}} \rightarrow-\frac{\alpha}{8 \pi}\left[\bar{u}_{p} W^{\mu} u_{n}\right]\left[\bar{u}_{e} O_{\mu} v_{\bar{\nu}}\right] \\
& \times \int_{0}^{1} d x 2 x \int \frac{d^{4} q}{\pi^{2} i}\left[\frac{4 m_{p}^{2}-4\left(k_{p} \cdot q\right)+q^{2}}{\left(\left(q-k_{p} x\right)^{2}-m_{p}^{2} x^{2}-\mu^{2}(1-x)\right)^{3}}-\frac{4\left(k_{p} \cdot q\right)+q^{2}}{\left(\left(q-k_{p} x\right)^{2}-m_{p}^{2} x^{2}-\Lambda^{2}(1-x)\right)^{3}}\right]= \\
& =\frac{\alpha}{8 \pi}\left[\bar{u}_{p} W^{\mu} u_{n}\right]\left[\bar{u}_{e} O_{\mu} v_{\bar{\nu}}\right] \int_{0}^{1} d x 2 x \int \frac{d^{4} q}{\pi^{2}}\left[\frac{m_{p}^{2}\left(4-4 x+x^{2}\right)-q^{2}}{\left(q^{2}+m_{p}^{2} x^{2}+\mu^{2}(1-x)\right)^{3}}-\frac{m_{p}^{2}\left(4-4 x+x^{2}\right)-q^{2}}{\left(q^{2}+m_{p}^{2} x^{2}+\Lambda^{2}(1-x)\right)^{3}}\right]= \\
& =\frac{\alpha}{8 \pi}\left[\bar{u}_{p} W^{\mu} u_{n}\right]\left[\bar{u}_{e} O_{\mu} v_{\bar{\nu}}\right] \int_{0}^{1} d x 2 x \int \frac{d^{4} q}{\pi^{2}}\left[\frac{m_{p}^{2}\left(4-4 x+2 x^{2}\right)}{\left(q^{2}+m_{p}^{2} x^{2}+\mu^{2}(1-x)\right)^{3}}-\frac{m_{p}^{2}\left(4-4 x+2 x^{2}\right)+\Lambda^{2}(1-x)}{\left(q^{2}+m_{p}^{2} x^{2}+\Lambda^{2}(1-x)\right)^{3}}\right.
\end{aligned}
$$




$$
\begin{aligned}
& \left.-\frac{1}{\left(q^{2}+m_{p}^{2} x^{2}+\mu^{2}(1-x)\right)^{2}}+\frac{1}{\left(q^{2}+m_{p}^{2} x^{2}+\Lambda^{2}(1-x)\right)^{2}}\right]=\frac{\alpha}{8 \pi}\left[\bar{u}_{p} W^{\mu} u_{n}\right]\left[\bar{u}_{e} O_{\mu} v_{\bar{\nu}}\right] \int_{0}^{1} d x 2 x\left\{\frac{m_{p}^{2}\left(2-2 x+x^{2}\right)}{m_{p}^{2} x^{2}+\mu^{2}(1-x)}\right. \\
& \left.-\frac{1}{2}-\ln \left[\frac{\Lambda^{2}(1-x)}{m_{p}^{2} x^{2}}\right]\right\} .
\end{aligned}
$$

Using the results, obtained in Appendix D, we define $\mathcal{M}_{p p}^{(\mathrm{SC})}$ as follows

$$
\mathcal{M}_{p p}^{(\mathrm{SC})}=\left[\bar{u}_{p} W^{\mu} u_{n}\right]\left[\bar{u}_{e} O_{\mu} v_{\bar{\nu}}\right] \frac{\alpha}{2 \pi}\left[-\frac{1}{2} \ell n\left(\frac{\Lambda}{m_{p}}\right)-\ell n\left(\frac{\mu}{m_{p}}\right)-\frac{3}{4}\right] .
$$

After the summation of the contributions the amplitude $\mathcal{M}_{\mathrm{RC}}^{(\mathrm{SC})}$ takes the form

$$
\begin{aligned}
& \mathcal{M}_{\mathrm{RC}}^{(\mathrm{SC})}=\left[\bar{u}_{p} W^{\mu} u_{n}\right] \frac{\alpha}{2 \pi}\left\{[ \overline { u } _ { e } O _ { \mu } v _ { \overline { \nu } } ] \left[\frac{3}{2} \ln \left(\frac{m_{p}}{m_{e}}\right)-\frac{11}{8}+2 \ln \left(\frac{\mu}{m_{e}}\right)\left[\frac{1}{2 \beta} \ln \left(\frac{1+\beta}{1-\beta}\right)-1\right]\right.\right. \\
& \left.\left.+\frac{1}{\beta} L\left(\frac{2 \beta}{1+\beta}\right)-\frac{1}{4 \beta} \ln ^{2}\left(\frac{1+\beta}{1-\beta}\right)+\frac{1}{2 \beta} \ln \left(\frac{1+\beta}{1-\beta}\right)\right]-\left[\bar{u}_{e} \gamma^{0} O_{\mu} v_{\bar{\nu}}\right] \frac{\sqrt{1-\beta^{2}}}{2 \beta} \ln \left(\frac{1+\beta}{1-\beta}\right)\right\} .
\end{aligned}
$$

Thus, the value of the constant $c_{S}$ is $c_{S}=-11 / 8$ and the radiative corrections do not depend on the ultra-violet cut-off. It is caused by the requirement of gauge invariance of the observable part of the radiative corrections to the amplitude of the continuum-state $\beta^{-}$-decay of the neutron.

Now let us proceed to calculating the contribution of $\delta \mathcal{M}_{\mathrm{RC}}^{(\mathrm{SLI})}$. The term $\mathcal{M}_{p e}^{(\mathrm{SLI})}$ is defined by

$$
\begin{aligned}
& \delta \mathcal{M}_{p e}^{(\mathrm{SLI})}=-\frac{\alpha}{4 \pi} \int \frac{d^{4} q}{\pi^{2} i} D_{\alpha \beta}(q) \frac{\left[\bar{u}_{e}\left(2 k_{e}^{\alpha}+\gamma^{\alpha} \hat{q}\right) O_{\mu} v_{\bar{\nu}}\right]}{q^{2}+2 k_{e} \cdot q+i 0}\left[\bar{u}_{p} T^{\beta \mu} u_{n}\right]=\frac{\alpha}{4 \pi}\left\{\left[\bar{u}_{p} W^{\mu} u_{n}\right]\left[\bar{u}_{e} O_{\mu} v_{\bar{\nu}}\right]\right. \\
& \times 3 \int \frac{d^{4} q}{\pi^{2} i} \frac{1}{q^{2}-2 k_{p} \cdot q+i 0} \frac{1}{q^{2}+2 k_{e} \cdot q+i 0}+2(\lambda+1)\left[\bar{u}_{p} R^{\mu} u_{n}\right]\left[\bar{u}_{e} O_{\mu} v_{\bar{\nu}}\right] \int \frac{d^{4} q}{\pi^{2} i} \frac{1}{q^{2}-2 k_{p} \cdot q+i 0} \frac{1}{q^{2}+2 k_{e} \cdot q+i 0} \\
& -2(\lambda+1)\left[\bar{u}_{p} R^{\alpha} u_{n}\right]\left[\bar{u}_{e} O^{\beta} v_{\bar{\nu}}\right] \int \frac{d^{4} q}{\pi^{2} i} \frac{q_{\alpha} q_{\beta}}{q^{2}+i 0} \frac{1}{q^{2}-2 k_{p} \cdot q+i 0} \frac{1}{q^{2}+2 k_{e} \cdot q+i 0} \\
& \left.\left.-2 i \int \frac{d^{4} q}{\pi^{2} i} \frac{1}{q^{2}+i 0} \frac{1}{q^{2}-2 k_{p} \cdot q+i 0} \frac{1}{q^{2}+2 k_{e} \cdot q+i 0}\left[\bar{u}_{p} \sigma_{\alpha \beta} k_{e}^{\alpha} q^{\beta} W^{\mu} u_{n}\right]\left[\bar{u}_{e} O_{\mu}\right) v_{\bar{\nu}}\right]\right\},
\end{aligned}
$$

where $R^{\lambda}=\gamma^{\lambda}\left(1+\gamma^{5}\right)$. Using the results, obtained in Appendix D (see Eq.(D-12), Eq.(D-28), Eq.(D-33) and Eq.(D-38) $)$ we obtain

$$
\begin{aligned}
& \delta \mathcal{M}_{p e}^{(\mathrm{SLI})}=\frac{\alpha}{2 \pi}\left[3 \ln \left(\frac{\Lambda}{m_{p}}\right)+\frac{3}{2}\right]\left[\bar{u}_{p} W^{\mu} u_{n}\right]\left[\bar{u}_{e} O_{\mu} v_{\bar{\nu}}\right] \\
& +\frac{\alpha}{2 \pi}(\lambda+1)\left[-2 \ln \left(\frac{\Lambda}{m_{p}}\right)-1\right]\left[\bar{u}_{p} R^{\mu} u_{n}\right]\left[\bar{u}_{e} O_{\mu} v_{\bar{\nu}}\right] \\
& +\frac{\alpha}{2 \pi}(\lambda+1)\left\{g_{\alpha \beta}\left[\frac{1}{2} \ln \left(\frac{\Lambda}{m_{p}}\right)+\frac{3}{4}\right]-\frac{1}{2} g_{o \alpha} g_{0 \beta}\right\}\left[\bar{u}_{p} R^{\alpha} u_{n}\right]\left[\bar{u}_{e} O^{\beta} v_{\bar{\nu}}\right] .
\end{aligned}
$$

For $\mathcal{M}_{e e}^{(\mathrm{SLI})}$ and $\mathcal{M}_{p p}^{(\mathrm{SLI})}$ we calculate the following expressions

$\mathcal{M}_{e e}^{(\mathrm{SLI})}=\left[\bar{u}_{p} W^{\mu} u_{n}\right]\left[\bar{u}_{e} O_{\mu} v_{\bar{\nu}}\right]\left\{\frac{Z_{2}^{(e)}-1}{2}+\frac{\alpha}{2 \pi}\left(-\ln \left(\frac{\Lambda}{m_{e}}\right)-2 \ln \left(\frac{\mu}{m_{e}}\right)-\frac{9}{4}\right)+\frac{\alpha}{2 \pi}\left(\frac{1}{2} \ln \left(\frac{\Lambda}{m_{e}}\right)+\ln \left(\frac{\mu}{m_{e}}\right)+\frac{9}{8}\right)\right\}=$

$=\left[\bar{u}_{p} W^{\mu} u_{n}\right]\left[\bar{u}_{e} O_{\mu} v_{\bar{\nu}}\right]\left\{\frac{Z_{2}^{(e)}-1}{2}-\frac{\alpha}{2 \pi}\left(\frac{1}{2} \ln \left(\frac{\Lambda}{m_{e}}\right)+\ln \left(\frac{\mu}{m_{e}}\right)+\frac{9}{8}\right)\right\}$

and

$\mathcal{M}_{p p}^{(\mathrm{SLI})}=\left[\bar{u}_{p} W^{\mu} u_{n}\right]\left[\bar{u}_{e} O_{\mu} v_{\bar{\nu}}\right]\left\{\frac{Z_{2}^{(p)}-1}{2}+\frac{\alpha}{2 \pi}\left(-\ln \left(\frac{\Lambda}{m_{p}}\right)-2 \ln \left(\frac{\mu}{m_{p}}\right)-\frac{9}{4}\right)+\frac{\alpha}{2 \pi}\left(\frac{1}{2} \ln \left(\frac{\Lambda}{m_{p}}\right)+\ln \left(\frac{\mu}{m_{p}}\right)+\frac{3}{4}\right)\right\}=$

$=\left[\bar{u}_{p} W^{\mu} u_{n}\right]\left[\bar{u}_{e} O_{\mu} v_{\bar{\nu}}\right]\left\{\frac{Z_{2}^{(e)}-1}{2}-\frac{\alpha}{2 \pi}\left(\frac{1}{2} \ln \left(\frac{\Lambda}{m_{p}}\right)+\ln \left(\frac{\mu}{m_{p}}\right)+\frac{3}{2}\right)\right\}$, 
respectively. Using the definition of the renormalisation constants $Z_{2}^{(e)}$ and $Z_{2}^{(p)}$ Eq. (D-25) we obtain

$$
\begin{aligned}
& \mathcal{M}_{e e}^{(\mathrm{SLI})}=0, \\
& \mathcal{M}_{p p}^{(\mathrm{SLI})}=\left[\bar{u}_{p} W^{\mu} u_{n}\right]\left[\bar{u}_{e} O_{\mu} v_{\bar{\nu}}\right] \frac{\alpha}{2 \pi}\left(-\frac{3}{8}\right) .
\end{aligned}
$$

The sum of the contributions to $\mathcal{M}_{\mathrm{RC}}^{(\mathrm{SLI})}$ gives one

$$
\begin{aligned}
& \mathcal{M}_{\mathrm{RC}}^{(\mathrm{SLI})}=\frac{\alpha}{2 \pi}\left[3 \ln \left(\frac{\Lambda}{m_{p}}\right)+\frac{3}{8}\right]\left[\bar{u}_{p} W^{\mu} u_{n}\right]\left[\bar{u}_{e} O_{\mu} v_{\bar{\nu}}\right] \\
& +\frac{\alpha}{2 \pi}(\lambda+1)\left[-2 \ln \left(\frac{\Lambda}{m_{p}}\right)-1\right]\left[\bar{u}_{p} R^{\mu} u_{n}\right]\left[\bar{u}_{e} O_{\mu} v_{\bar{\nu}}\right] \\
& +\frac{\alpha}{2 \pi}(\lambda+1)\left\{g_{\alpha \beta}\left[\frac{1}{2} \ell n\left(\frac{\Lambda}{m_{p}}\right)+\frac{3}{4}\right]-\frac{1}{2} g_{o \alpha} g_{0 \beta}\right\}\left[\bar{u}_{p} R^{\alpha} u_{n}\right]\left[\bar{u}_{e} O^{\beta} v_{\bar{\nu}}\right] .
\end{aligned}
$$

This shows that gauge non-invariant contributions do not depend on the electron energy $E_{e}$ and the infrared cut-off $\mu$ and may be fully absorbed by the renormalisation constants of the Fermi and axial coupling constants (see Eq. (D-48) and Eq.(D-49) $)$.

In order to prove the correctness of the term $-3 / 8$ in Eq. (D-59), giving the contribution to the observable radiative corrections to the lifetime of the neutron, we propose to sum up the contributions of $\mathcal{M}_{\mathrm{RC}}^{(\mathrm{SC})}$ and the terms, proportional to $\left[\bar{u}_{p} W^{\mu} u_{n}\right]\left[\bar{u}_{e} O_{\mu} v_{\bar{\nu}}\right]$ from $\mathcal{M}_{\mathrm{RC}}^{(\mathrm{SLI})}$, which we denote as $\overline{\mathcal{M}}_{\mathrm{RC}}^{(\mathrm{SLI})}$. This gives

$$
\mathcal{M}_{\mathrm{RC}}=\mathcal{M}_{\mathrm{RC}}^{(\mathrm{SC})}+\overline{\mathcal{M}}_{\mathrm{RC}}^{(\mathrm{SLI})}=\frac{\alpha}{2 \pi}\left\{\left[\bar{u}_{p} W^{\mu} u_{n}\right]\left[\bar{u}_{e} O_{\mu} v_{\bar{\nu}}\right] F\left(E_{e}, \mu\right)-\left[\bar{u}_{p} W^{\mu} u_{n}\right]\left[\bar{u}_{e} \gamma^{0} O_{\mu} v_{\bar{\nu}}\right] \frac{\sqrt{1-\beta^{2}}}{2 \beta} \ell n\left(\frac{1+\beta}{1-\beta}\right),\right.
$$

where the function $F\left(E_{e}, \mu\right)$ takes the form

$$
\begin{aligned}
F\left(E_{e}, \mu\right) & =3 \ln \left(\frac{\Lambda}{m_{p}}\right)+\frac{3}{2} \ln \left(\frac{m_{p}}{m_{e}}\right)-\frac{1}{4}+2 \ln \left(\frac{\mu}{m_{e}}\right)\left[\frac{1}{2 \beta} \ln \left(\frac{1+\beta}{1-\beta}\right)-1\right]+\frac{1}{\beta} L\left(\frac{2 \beta}{1+\beta}\right) \\
& -\frac{1}{4 \beta} \ln ^{2}\left(\frac{1+\beta}{1-\beta}\right)+\frac{1}{2 \beta} \ln \left(\frac{1+\beta}{1-\beta}\right) .
\end{aligned}
$$

The contribution of the radiative corrections to the lifetime of the neutron is defined by the function

$$
\begin{aligned}
g\left(E_{e}\right) & =\lim _{\mu \rightarrow 0}\left[F\left(E_{e}, \mu\right)+g_{\beta_{c}^{-}}^{(1)}\left(E_{e}, \mu\right)\right]= \\
& =3 \ln \left(\frac{\Lambda}{m_{p}}\right)+\frac{3}{2} \ln \left(\frac{m_{p}}{m_{e}}\right)+\frac{3}{4}+2\left[\frac{1}{2 \beta} \ln \left(\frac{1+\beta}{1-\beta}\right)-1\right]\left[\ln \left(\frac{2\left(E_{0}-E_{e}\right)}{m_{e}}\right)-\frac{3}{2}+\frac{1}{3} \frac{E_{0}-E_{e}}{E_{e}}\right] \\
& +\frac{2}{\beta} L\left(\frac{2 \beta}{1+\beta}\right)+\frac{1}{2 \beta} \ln \left(\frac{1+\beta}{1-\beta}\right)\left[\left(1+\beta^{2}\right)+\frac{1}{12} \frac{\left(E_{0}-E_{e}\right)^{2}}{E_{e}^{2}}-\ln \left(\frac{1+\beta}{1-\beta}\right)\right]
\end{aligned}
$$

where the function $g_{\beta_{c}^{-}}^{(1)}\left(E_{e}, \mu\right)$ is given by Eq.(B-28) and describes the contribution of the radiative $\beta^{-}-$decay of the neutron.

The function Eq. (F-30) reproduces our result, obtained in Appendix D (see Eq. (D-60)). Then, being multiplied by $(\alpha / \pi)$, it reproduces also the radiative corrections to the lifetime of the neutron, calculated by Kinoshita and Sirlin [15]. This corroborates the correctness of our calculation of $\mathcal{M}_{\mathrm{RC}}^{(\mathrm{SC})}$ and $\mathcal{M}_{\mathrm{RC}}^{(\mathrm{SLI})}$.

Thus, the analysis of the radiative corrections to the neutron $\beta^{-}$-decay, carried out in this Appendix, confirms Sirlin's assertion that the requirement of gauge invariance of the amplitude of the radiative corrections defines unambiguously the observable radiative corrections to the lifetime of the neutron. They are independent of the axial coupling constant $\lambda$, i.e. of strong low-energy interactions. The part of radiative corrections depending on the axial coupling constant $\lambda$, i.e. on strong low-energy interactions, is unobservable and absorbed by renormalisation constants of the Fermi and axial coupling constants.

\section{Appendix G: Contribution of weak lepton-nucleon couplings beyond SM to correlation coefficients of neutron $\beta^{-}$-decay}

In this Appendix we take into account the contributions of the weak lepton-nucleon interactions beyond the SM with left-handed and right-handed neutrinos. For this aim we use the following Hamiltonian of phenomenological 
weak lepton-nucleon interactions [4] - []

$$
\begin{aligned}
\mathcal{H}_{W}(x) & =\frac{G_{F}}{\sqrt{2}} V_{u d}\left\{\left[\bar{\psi}_{p}(x) \gamma_{\mu} \psi_{n}(x)\right]\left[\bar{\psi}_{e}(x) \gamma^{\mu}\left(C_{V}+\bar{C}_{V} \gamma^{5}\right) \psi_{\nu_{e}}(x)\right]+\left[\bar{\psi}_{p}(x) \gamma_{\mu} \gamma^{5} \psi_{n}(x)\right]\left[\bar{\psi}_{e}(x) \gamma^{\mu}\left(\bar{C}_{A}+C_{A} \gamma^{5}\right) \psi_{\nu_{e}}(x)\right]\right. \\
& +\left[\bar{\psi}_{p}(x) \psi_{n}(x)\right]\left[\bar{\psi}_{e}(x)\left(C_{S}+\bar{C}_{S} \gamma^{5}\right) \psi_{\nu_{e}}(x)\right]+\left[\bar{\psi}_{p}(x) \gamma^{5} \psi_{n}(x)\right]\left[\bar{\psi}_{e}(x)\left(C_{P}+\bar{C}_{P} \gamma^{5}\right) \psi_{\nu_{e}}(x)\right] \\
& +\frac{1}{2}\left[\bar{\psi}_{p}(x) \sigma^{\mu \nu} \gamma^{5} \psi_{n}(x)\right]\left[\bar{\psi}_{e}(x) \sigma_{\mu \nu}\left(\bar{C}_{T}+C_{T} \gamma^{5}\right) \psi_{\nu_{e}}(x)\right\}
\end{aligned}
$$

This is the most general form of the effective low-energy weak interactions, where the coupling constants $C_{i}$ and $\bar{C}_{i}$ for $i=V, A, S, P$ and $T$ can be induced by the left-handed and right-handed hadronic and leptonic currents [4]- 7] and supersymmetric interactions [3] as well. They are related to the coupling constants, analogous to those which were introduced by Herczeg [6], as follows

$$
\begin{aligned}
C_{V} & =1+a_{L L}^{h}+a_{L R}^{h}+a_{R R}^{h}+a_{R L}^{h}, \\
\bar{C}_{V} & =-1-a_{L L}^{h}-a_{L R}^{h}+a_{R R}^{h}+a_{R L}^{h}, \\
C_{A} & =-\lambda+a_{L L}^{h}-a_{L R}^{h}+a_{R R}^{h}-a_{R L}^{h}, \\
\bar{C}_{A} & =\lambda-a_{L L}^{h}+a_{L R}^{h}+a_{R R}^{h}-a_{R L}^{h}, \\
C_{S} & =A_{L L}^{h}+A_{L R}^{h}+A_{R R}^{h}+A_{R L}^{h}, \\
\bar{C}_{S} & =-A_{L L}^{h}-A_{L R}^{h}+A_{R R}^{h}+A_{R L}^{h}, \\
C_{P} & =-A_{L L}^{h}+A_{L R}^{h}+A_{R R}^{h}-A_{R L}^{h}, \\
\bar{C}_{P} & =A_{L L}^{h}-A_{L R}^{h}+A_{R R}^{h}-A_{R L}^{h}, \\
C_{T} & =2\left(\alpha_{L L}^{h}+\alpha_{R R}^{h}\right), \\
\bar{C}_{T} & =2\left(-\alpha_{L L}^{h}+\alpha_{R R}^{h}\right),
\end{aligned}
$$

where the index $h$ means that the coupling constants are introduced at the hadronic level but not at the quark level as it has been done by Herczeg [6]. In addition in comparison with Herczeg [6] we have taken away the common factor $G_{F} V_{u d} / \sqrt{2}$ and defined the coupling constants $a_{L L}^{h}$ and $a_{L R}^{h}$ as deviations from the coupling constants of the SM [105]. Analogous to Herczeg [6], the Hamiltonian of phenomenological lepton-nucleon weak interactions beyond the SM may be written in the following form [105]

$$
\begin{aligned}
& \mathcal{H}_{W}(x)=\frac{G_{F}}{\sqrt{2}} V_{u d}\left\{[ \overline { \psi } _ { e } ( x ) \gamma _ { \mu } ( 1 - \gamma ^ { 5 } ) \psi _ { \nu _ { e } } ( x ) ] \left[\left(\frac{1-\lambda}{2}+a_{L L}^{h}\right)\left[\bar{\psi}_{p}(x) \gamma^{\mu}\left(1-\gamma^{5}\right) \psi_{n}(x)\right]\right.\right. \\
& \left.+\left(\frac{1+\lambda}{2}+a_{L R}^{h}\right)\left[\bar{\psi}_{p}(x) \gamma^{\mu}\left(1+\gamma^{5}\right) \psi_{n}(x)\right]\right]+\left[\bar{\psi}_{e}(x) \gamma_{\mu}\left(1+\gamma^{5}\right) \psi_{\nu_{e}}(x)\right]\left(a_{R L}^{h}\left[\bar{\psi}_{p}(x) \gamma^{\mu}\left(1-\gamma^{5}\right) \psi_{n}(x)\right]\right. \\
& \left.+a_{R R}^{h}\left[\bar{\psi}_{p}(x) \gamma^{\mu}\left(1+\gamma^{5}\right) \psi_{n}(x)\right]\right)+\left[\bar{\psi}_{e}(x)\left(1-\gamma^{5}\right) \psi_{\nu_{e}}(x)\right]\left(A_{L L}^{h}\left[\bar{\psi}_{p}(x)\left(1-\gamma^{5}\right) \psi_{n}(x)\right]+A_{L R}^{h}\left[\bar{\psi}_{p}(x)\left(1+\gamma^{5}\right) \psi_{n}(x)\right]\right) \\
& \left.+\left[\bar{\psi}_{e}(x)\left(1+\gamma^{5}\right) \psi_{\nu_{e}}(x)\right]\left(A_{R L}^{h}\left[\bar{\psi}_{p}(x)\left(1-\gamma^{5}\right) \psi_{n}(x)\right]\right)+A_{R R}^{h}\left[\bar{\psi}_{p}(x)\left(1+\gamma^{5}\right) \psi_{n}(x)\right]\right)+\frac{1}{2} \alpha_{L L}^{h}\left[\bar{\psi}_{e}(x) \sigma_{\mu \nu}\left(1-\gamma^{5}\right) \psi_{\nu_{e}}(x)\right] \\
& \left.\times\left[\bar{\psi}_{p}(x) \sigma^{\mu \nu}\left(1-\gamma^{5}\right) \psi_{n}(x)\right]+\frac{1}{2} \alpha_{R R}^{h}\left[\bar{\psi}_{e}(x) \sigma_{\mu \nu}\left(1+\gamma^{5}\right) \psi_{\nu_{e}}(x)\right]\left[\bar{\psi}_{p}(x) \sigma^{\mu \nu}\left(1+\gamma^{5}\right) \psi_{n}(x)\right]\right\} .
\end{aligned}
$$

In order to express the coupling constants $C_{T}$ and $\bar{C}_{T}$ in terms of the coupling constants $\alpha_{L L}^{h}$ and $\alpha_{R R}^{h}$ we have used the relation $\sigma_{\mu \nu} \gamma^{5}=\frac{i}{2} \varepsilon_{\mu \nu \alpha \beta} \sigma^{\alpha \beta}[35]$.

The $\mathrm{SM}$ is defined by the coupling constants $C_{S}=\bar{C}_{S}=C_{P}=\bar{C}_{P}=C_{T}=\bar{C}_{T}=0, C_{V}=-\bar{C}_{V}=1$ and $C_{A}=-\bar{C}_{A}=-\lambda$. The coupling constants $a_{i j}^{h}, A_{i j}^{h}$ and $\alpha_{j j}^{h}$ for $i(j)=L$ or $R$ are induced by interactions beyond the SM.

The contributions of the pseudoscalar weak interactions with the coupling constants $C_{P}$ and $\bar{C}_{P}$ to the amplitude of the neutron $\beta^{-}$-decay are of order $O\left(C_{P} / M\right)$ and $O\left(\bar{C}_{P} / M\right)$, which are caused by the proton recoil. Since these corrections are of order $10^{-6}$ or even smaller, the contributions of the pseudoscalar weak interactions with the coupling constants $C_{P}$ and $\bar{C}_{P}$ to the correlation coefficients of the neutron $\beta^{-}$-decay may be neglected in comparison with contributions of order $10^{-4}$ of our interest.

The amplitude of the continuum-state $\beta^{-}$-decay of the neutron, calculated with the Hamiltonian of weak interactions Eq. G-1 to leading order in the large proton mass expansion, takes the form

$$
\begin{aligned}
M\left(n \rightarrow p e^{-} \bar{\nu}_{e}\right)= & -2 m_{n} \frac{G_{F}}{\sqrt{2}} V_{u d}\left\{\left[\varphi_{p}^{\dagger} \varphi_{n}\right]\left[\bar{u}_{e} \gamma^{0}\left(C_{V}+\bar{C}_{V} \gamma^{5}\right) v_{\bar{\nu}}\right]-\left[\varphi_{p}^{\dagger} \vec{\sigma} \varphi_{n}\right] \cdot\left[\bar{u}_{e} \vec{\gamma}\left(\bar{C}_{A}+C_{A} \gamma^{5}\right) v_{\bar{\nu}}\right]\right. \\
& +\left[\varphi_{p}^{\dagger} \varphi_{n}\right]\left[\bar{u}_{e}\left(C_{S}+\bar{C}_{S} \gamma^{5}\right) v_{\bar{\nu}}\right]+\left[\varphi_{p}^{\dagger} \vec{\sigma} \varphi_{n}\right] \cdot\left[\bar{u}_{e} \gamma^{0} \vec{\gamma}_{\left.\left.\left(\bar{C}_{T}+C_{T} \gamma^{5}\right) v_{\bar{\nu}}\right]\right\}}\right.
\end{aligned}
$$


The hermitian conjugate amplitude is

$$
\begin{aligned}
M^{\dagger}\left(n \rightarrow p e^{-} \bar{\nu}_{e}\right)= & -2 m_{n} \frac{G_{F}}{\sqrt{2}} V_{u d}^{*}\left\{\left[\varphi_{n}^{\dagger} \varphi_{p}\right]\left[\bar{v}_{\bar{\nu}} \gamma^{0}\left(C_{V}^{*}+\bar{C}_{V}^{*} \gamma^{5}\right) u_{e}\right]-\left[\varphi_{n}^{\dagger} \vec{\sigma} \varphi_{p}\right] \cdot\left[\bar{v}_{\bar{\nu}} \vec{\gamma}\left(\bar{C}_{A}^{*}+C_{A}^{*} \gamma^{5}\right) u_{e}\right]\right. \\
& \left.+\left[\varphi_{n}^{\dagger} \varphi_{p}\right]\left[\bar{v}_{\bar{\nu}}\left(C_{S}^{*}-\bar{C}_{S}^{*} \gamma^{5}\right) u_{e}\right]-\left[\varphi_{n}^{\dagger} \vec{\sigma} \varphi_{p}\right] \cdot\left[\bar{v}_{\bar{\nu}} \gamma^{0} \vec{\gamma}\left(\bar{C}_{T}^{*}-C_{T}^{*} \gamma^{5}\right) u_{e}\right]\right\}
\end{aligned}
$$

The squared absolute value of the amplitude Eq. (G-4) for the polarized neutron and unpolarised proton and electron is given by

$$
\sum_{\text {pol }}\left|M\left(n \rightarrow p e^{-} \bar{\nu}_{e}\right)\right|^{2}=8 m_{n}^{2} G_{F}^{2}\left|V_{u d}\right|^{2} E_{e} E \xi\left(1+b \frac{m_{e}}{E_{e}}+a \frac{\vec{k}_{e} \cdot \vec{k}}{E_{e} E}+A \frac{\vec{\xi}_{n} \cdot \vec{k}_{e}}{E_{e}}+B \frac{\vec{\xi}_{n} \cdot \vec{k}}{E}+D \frac{\vec{\xi}_{n} \cdot\left(\vec{k}_{e} \times \vec{k}\right)}{E_{e} E}\right)
$$

The correlation coefficients of the neutron $\beta^{-}$-decay, expressed in terms of the phenomenological coupling constants $C_{j}$ and $\bar{C}_{j}$ for $j=V, A, S$ and $T$ and calculated to leading order in the large $M$ expansion, are equal to

$$
\begin{aligned}
\xi= & \left|C_{V}\right|^{2}+\left|\bar{C}_{V}\right|^{2}+3\left|C_{A}\right|^{2}+3\left|\bar{C}_{A}\right|^{2}+\left|C_{S}\right|^{2}+\left|\bar{C}_{S}\right|^{2}+3\left|C_{T}\right|^{2}+3\left|\bar{C}_{T}\right|^{2}, \\
\xi a= & \left|C_{V}\right|^{2}+\left|\bar{C}_{V}\right|^{2}-\left|C_{A}\right|^{2}-\left|\bar{C}_{A}\right|^{2}+\left|C_{T}\right|^{2}+\left|\bar{C}_{T}\right|^{2}-\left|C_{S}\right|^{2}-\left|\bar{C}_{S}\right|^{2}, \\
\xi b= & 2 \operatorname{Re}\left(\left(C_{V} C_{S}^{*}+\bar{C}_{V} \bar{C}_{S}^{*}\right)-3\left(C_{A} C_{T}^{*}+\bar{C}_{A} \bar{C}_{T}^{*}\right)\right), \\
\xi A= & 2 \operatorname{Re}\left(2 C_{A} \bar{C}_{A}^{*}-2 C_{T} \bar{C}_{T}^{*}-\left(C_{V} \bar{C}_{A}^{*}+\bar{C}_{V} C_{A}^{*}\right)-\left(C_{S} \bar{C}_{T}^{*}+\bar{C}_{S} C_{T}^{*}\right)\right), \\
\xi B= & -2 \operatorname{Re}\left(2 C_{A} \bar{C}_{A}^{*}+2 C_{T} \bar{C}_{T}^{*}+\left(C_{V} \bar{C}_{A}^{*}+\bar{C}_{V} C_{A}^{*}\right)-\left(C_{S} \bar{C}_{T}^{*}+\bar{C}_{S} C_{T}^{*}\right)\right) \\
& +2 \operatorname{Re}\left(\left(C_{V} \bar{C}_{T}^{*}+\bar{C}_{V} C_{T}^{*}\right)-\left(C_{A} \bar{C}_{S}^{*}+\bar{C}_{A} C_{S}^{*}\right)+2\left(C_{A} \bar{C}_{T}^{*}+\bar{C}_{A} C_{T}^{*}\right)\right) \frac{m_{e}}{E_{e}}, \\
\xi D= & 2 \operatorname{Im}\left(\left(C_{V} C_{A}^{*}+\bar{C}_{V} \bar{C}_{A}^{*}\right)+\left(C_{S} C_{T}^{*}+\bar{C}_{S} \bar{C}_{T}^{*}\right)\right) .
\end{aligned}
$$

The correlation coefficients Eq. [G-7] reproduce well the structure of the correlation coefficients, calculated in [4]-7]. In the linear approximation with respect to the deviations of the coupling constants $C_{j}$ and $\bar{C}_{j}$ for $j=V, A, S$ and $T$ from the coupling constants of the SM the correlation coefficients $\xi, a, b, A, B$ and $D$ read

$$
\begin{aligned}
\xi= & 2\left(1+3 \lambda^{2}\right)\left[1+\frac{1}{1+3 \lambda^{2}}\left(\operatorname{Re}\left(\delta C_{V}-\delta \bar{C}_{V}\right)-3 \lambda \operatorname{Re}\left(\delta C_{A}-\delta \bar{C}_{A}\right)\right)\right] \\
a= & a_{0}+\frac{1}{1+3 \lambda^{2}}\left[\operatorname{Re}\left(\delta C_{V}-\delta \bar{C}_{V}\right)+\lambda \operatorname{Re}\left(\delta C_{A}-\delta \bar{C}_{A}\right)-a_{0}\left(\operatorname{Re}\left(\delta C_{V}-\delta \bar{C}_{V}\right)-3 \lambda \operatorname{Re}\left(\delta C_{A}-\delta \bar{C}_{A}\right)\right)\right] \\
b= & \frac{1}{1+3 \lambda^{2}}\left(\operatorname{Re}\left(C_{S}-\bar{C}_{S}\right)+3 \lambda \operatorname{Re}\left(C_{T}-\bar{C}_{T}\right)\right), \\
A= & A_{0}+\frac{1}{1+3 \lambda^{2}}\left[-\lambda \operatorname{Re}\left(\delta C_{V}-\delta \bar{C}_{V}\right)+(1+2 \lambda) \operatorname{Re}\left(\delta C_{A}-\delta \bar{C}_{A}\right)-A_{0}\left(\operatorname{Re}\left(\delta C_{V}-\delta \bar{C}_{V}\right)-3 \lambda \operatorname{Re}\left(\delta C_{A}-\delta \bar{C}_{A}\right)\right)\right], \\
B= & B_{0}+\frac{1}{1+3 \lambda^{2}}\left[-\lambda \operatorname{Re}\left(\delta C_{V}-\delta \bar{C}_{V}\right)+(1-2 \lambda) \operatorname{Re}\left(\delta C_{A}-\delta \bar{C}_{A}\right)-B_{0}\left(\operatorname{Re}\left(\delta C_{V}-\delta \bar{C}_{V}\right)-3 \lambda \operatorname{Re}\left(\delta C_{A}-\delta \bar{C}_{A}\right)\right)\right. \\
& \left.+\left(-\lambda \operatorname{Re}\left(C_{S}-\bar{C}_{S}\right)-(1-2 \lambda) \operatorname{Re}\left(C_{T}-\bar{C}_{T}\right)\right) \frac{m_{e}}{E_{e}}\right] \\
D= & \frac{1}{1+3 \lambda^{2}}\left(-\lambda \operatorname{Im}\left(\delta C_{V}-\delta \bar{C}_{V}\right)-\operatorname{Im}\left(\delta C_{A}-\delta \bar{C}_{A}\right)\right),
\end{aligned}
$$

where the correlation coefficients $a_{0}, A_{0}$ and $B_{0}$ are defined in Eq.(8) and the phenomenological coupling constants $C_{J}$ and $\bar{C}_{j}$ for $j=V, A$ are taken in the form $C_{V}=1+\delta C_{V}, \bar{C}_{V}=-1+\delta \bar{C}_{V}, C_{A}=-\lambda+\delta C_{A}$ and $\bar{C}_{A}=\lambda+\delta \bar{C}_{A}$. The coupling constants $\delta C_{j}$ and $\delta \bar{C}_{j}$ for $j=V, A$ and the coupling constants $C_{j}$ and $\bar{C}_{j}$ for $j=S, T$ are caused by interactions beyond the SM. For the analysis of contributions of order $10^{-4}$ beyond the SM we may maintain the corrections, caused deviations of the phenomenological coupling constants $\bar{C}_{j}$ for $j=V, A, S$ and $T$ from the coupling constants of the SM, to linear order only.

In terms of the correlation coefficients Eq.(G-7) the rate of the neutron $\beta^{-}$-decay is equal to

$$
\lambda_{n}=\xi \frac{G_{F}^{2}\left|V_{u d}\right|^{2}}{4 \pi^{3}} f_{n}\left(E_{0}, Z=1\right)\left(1+b\left\langle\frac{m_{e}}{E_{e}}\right\rangle\right)
$$


where the Fermi integral $f_{n}\left(E_{0}, Z=1\right)$ is given by

$$
f_{n}\left(E_{0}, Z=1\right)=\int_{m_{e}}^{E_{0}}\left(E_{0}-E_{e}\right)^{2} \sqrt{E_{e}^{2}-m_{e}^{2}} E_{e} F\left(E_{e}, Z=1\right) d E_{e}
$$

The correlation coefficients of the neutron $\beta^{-}$-decay, calculated in this paper with the contributions of the "weak magnetism", the proton recoil, radiative corrections and contributions of interactions beyond the SM, are equal to

$$
\begin{aligned}
\zeta\left(E_{e}\right) & =\zeta_{\mathrm{SM}}\left(E_{e}\right)+\zeta_{\mathrm{NP}}\left(E_{e}\right), \\
a\left(E_{e}\right) & =a_{\mathrm{SM}}\left(E_{e}\right)+a_{\mathrm{NP}}\left(E_{e}\right), \\
A\left(E_{e}\right) & =A_{\mathrm{SM}}\left(E_{e}\right)+A_{\mathrm{NP}}\left(E_{e}\right), \\
B\left(E_{e}\right) & =B_{\mathrm{SM}}\left(E_{e}\right)+B_{\mathrm{NP}}\left(E_{e}\right), \\
D\left(E_{e}\right) & =D_{\mathrm{SM}}\left(E_{e}\right)+D_{\mathrm{NP}}\left(E_{e}\right),
\end{aligned}
$$

where the correlation coefficients $\zeta_{\mathrm{SM}}\left(E_{e}\right), a_{\mathrm{SM}}\left(E_{e}\right), A_{\mathrm{SM}}\left(E_{e}\right)$ and $B_{\mathrm{SM}}\left(E_{e}\right)$ are given by Eqs. (10) - (13), respectively. They are calculated within the SM with the contributions of the "weak magnetism", the proton recoil and radiative corrections. The correlation coefficient $D_{\mathrm{SM}}\left(E_{e}\right)$ has been calculated in [54]-[58]. For the electron kinetic energies $250 \mathrm{keV} \leq T_{e} \leq 455 \mathrm{keV}$ and the axial coupling constant $\lambda=-1,2750$ one may estimate that $D_{\mathrm{SM}}\left(E_{e}\right) \sim 10^{-5}[54]$. The correlations coefficients $\zeta_{\mathrm{NP}}\left(E_{e}\right), a_{\mathrm{NP}}\left(E_{e}\right), A_{\mathrm{NP}}\left(E_{e}\right), B_{\mathrm{NP}}\left(E_{e}\right)$ and $D_{\mathrm{NP}}\left(E_{e}\right)$ are defined by the contributions of interactions beyond the SM or a new physics (NP). They are taken to linear approximation with respect to the deviations of the phenomenological coupling constants $C_{j}$ and $\bar{C}_{j}$ for $j=V, A, S$ and $T$ from the coupling constants of the SM

$$
\begin{aligned}
\zeta_{\mathrm{NP}}\left(E_{e}\right) & =\frac{1}{1+3 \lambda^{2}}\left(\operatorname{Re}\left(\delta C_{V}-\delta \bar{C}_{V}\right)-3 \lambda \operatorname{Re}\left(\delta C_{A}-\delta \bar{C}_{A}\right)\right)+b_{F} \frac{m_{e}}{E_{e}} \\
a_{\mathrm{NP}}\left(E_{e}\right) & =\frac{1}{1+3 \lambda^{2}}\left(\operatorname{Re}\left(\delta C_{V}-\delta \bar{C}_{V}\right)+\lambda \operatorname{Re}\left(\delta C_{A}-\delta \bar{C}_{A}\right)\right. \\
& -a_{0}\left(\frac{1}{1+3 \lambda^{2}}\left(\operatorname{Re}\left(\delta C_{V}-\delta \bar{C}_{V}\right)-3 \lambda \operatorname{Re}\left(\delta C_{A}-\delta \bar{C}_{A}\right)\right)+b_{F} \frac{m_{e}}{E_{e}}\right) \\
A_{\mathrm{NP}}\left(E_{e}\right) & =\frac{1}{1+3 \lambda^{2}}\left(-\lambda \operatorname{Re}\left(\delta C_{V}-\delta \bar{C}_{V}\right)+(1+2 \lambda) \operatorname{Re}\left(\delta C_{A}-\delta \bar{C}_{A}\right)\right) \\
& -A_{0}\left(\frac{1}{1+3 \lambda^{2}}\left(\operatorname{Re}\left(\delta C_{V}-\delta \bar{C}_{V}\right)-3 \lambda \operatorname{Re}\left(\delta C_{A}-\delta \bar{C}_{A}\right)\right)+b_{F} \frac{m_{e}}{E_{e}}\right) \\
B_{\mathrm{NP}}\left(E_{e}\right) & =\frac{1}{1+3 \lambda^{2}} \operatorname{Re}\left(-\lambda \operatorname{Re}\left(\delta C_{V}-\delta \bar{C}_{V}\right)+(1-2 \lambda) \operatorname{Re}\left(\delta C_{A}-\delta \bar{C}_{A}\right)\right) \\
& +\frac{1}{1+3 \lambda^{2}}\left(-\lambda \operatorname{Re}\left(C_{S}-\bar{C}_{S}\right)-(1-2 \lambda) \operatorname{Re}\left(C_{T}-\bar{C}_{T}\right)\right) \frac{m_{e}}{E_{e}} \\
& -B_{0}\left(\frac{1}{1+3 \lambda^{2}}\left(\operatorname{Re}\left(\delta C_{V}-\delta \bar{C}_{V}\right)-3 \lambda \operatorname{Re}\left(\delta C_{A}-\delta \bar{C}_{A}\right)\right)+b_{F} \frac{m_{e}}{E_{e}}\right) \\
D_{\mathrm{NP}}\left(E_{e}\right) & =\frac{1}{1+3 \lambda^{2}}\left(-\lambda \operatorname{Im}\left(\delta C_{V}-\delta \bar{C}_{V}\right)-\operatorname{Im}\left(\delta C_{A}-\delta \bar{C}_{A}\right)\right)
\end{aligned}
$$

where $b_{F}$ is the Fierz term [1, 2, 4, 7], defined by

$$
b_{F}=\frac{1}{1+3 \lambda^{2}}\left(\operatorname{Re}\left(C_{S}-\bar{C}_{S}\right)+3 \lambda \operatorname{Re}\left(C_{T}-\bar{C}_{T}\right)\right) .
$$

The rate of the neutron $\beta^{-}$-decay, corrected by the contributions of interactions beyond the SM, takes the form

$$
\lambda_{n}=\left(\lambda_{n}\right)_{\mathrm{SM}}\left(1+\frac{1}{1+3 \lambda^{2}}\left(\operatorname{Re}\left(\delta C_{V}-\delta \bar{C}_{V}\right)-3 \lambda \operatorname{Re}\left(\delta C_{A}-\delta \bar{C}_{A}\right)\right)+b_{F}\left\langle\frac{m_{e}}{E_{e}}\right\rangle_{\mathrm{SM}}\right),
$$

where $\left(\lambda_{n}\right)_{\mathrm{SM}}$ is defined by Eq.(40) and $\left\langle m_{e} / E_{e}\right\rangle_{\mathrm{SM}}$ is the average value, calculated with the electron-energy spectrum Eq.(D-59). The lifetime of the neutron is equal to

$$
\tau_{n}=\left(\tau_{n}\right)_{\mathrm{SM}}\left(1-\frac{1}{1+3 \lambda^{2}}\left(\operatorname{Re}\left(\delta C_{V}-\delta \bar{C}_{V}\right)-3 \lambda \operatorname{Re}\left(\delta C_{A}-\delta \bar{C}_{A}\right)\right)-b_{F}\left\langle\frac{m_{e}}{E_{e}}\right\rangle_{\mathrm{SM}}\right) .
$$

In terms of the correlation coefficients Eq. (G-11) and Eq. G-12 the analysis of the sensitivity of the asymmetries $A_{\exp }\left(E_{e}\right), B_{\exp }\left(E_{e}\right)$ and $C_{\text {exp }}$, the proton-electron energy distribution $a\left(E_{e}, T_{p}\right)$, the proton-energy spectrum $a\left(T_{p}\right)$ and the lifetime of the neutron $\tau_{n}$ is given in section [X] 


\section{Appendix H: Contribution of proton recoil, caused by electron-proton final state Coulomb interaction}

In this Appendix we calculate the contribution of the proton recoil, caused by the electron-proton Coulomb interaction in the final state of the decay. A velocity of a relative motion of the electron-proton pair is equal to

$$
\vec{v}=\frac{\vec{k}_{p}}{m_{p}}-\frac{\vec{k}_{e}}{E_{e}}
$$

The Coulomb corrections, caused by a proton recoil and calculated to order $\alpha / M$, change the Fermi function Eq. (5) as follows

$$
F\left(E_{e}, Z=1\right) \rightarrow F\left(E_{e}, Z=1\right)\left(1-\frac{\pi \alpha}{\beta} \frac{E_{e}}{M}-\frac{\pi \alpha}{\beta^{3}} \frac{E_{0}-E_{e}}{M} \frac{\vec{k}_{e} \cdot \vec{k}}{E_{e} E}\right) .
$$

As a result the function $\zeta\left(E_{e}\right)$ acquires the following correction

$$
\zeta\left(E_{e}\right) \rightarrow \zeta\left(E_{e}\right) \eta\left(E_{e}\right)=\zeta\left(E_{e}\right)\left(1-\frac{\pi \alpha}{\beta} \frac{E_{e}}{M}\left(1+\frac{1}{3} a_{0} \frac{E_{0}-E_{e}}{E_{e}}\right)\right) .
$$

The function $\eta\left(E_{e}\right)$, averaged over the electron energy spectrum $\rho_{\beta_{c}^{-}}\left(E_{e}\right)$ Eq.(D-59) and equal to $\left\langle\eta\left(E_{e}\right)\right\rangle=1-$ $2.7 \times 10^{-5}$, defines a correction to the lifetime of the neutron of order $10^{-5}$. The correlation coefficients acquire the following corrections

$$
\begin{aligned}
\delta a\left(E_{e}\right) & =\frac{1}{3} a_{0}^{2} \frac{\pi \alpha}{\beta} \frac{E_{0}-E_{e}}{M}-\frac{\pi \alpha}{\beta^{3}} \frac{E_{0}-E_{e}}{M}, \delta A\left(E_{e}\right)=\frac{1}{3} a_{0} A_{0} \frac{\pi \alpha}{\beta} \frac{E_{0}-E_{e}}{M}, \delta B\left(E_{e}\right)=\frac{1}{3} a_{0} B_{0} \frac{\pi \alpha}{\beta} \frac{E_{0}-E_{e}}{M}, \\
\delta K_{n}\left(E_{e}\right) & =-A_{0} \frac{\pi \alpha}{\beta^{3}} \frac{E-E_{e}}{M}, \delta Q_{n}\left(E_{e}\right)=-B_{0} \frac{\pi \alpha}{\beta^{3}} \frac{E-E_{e}}{M} .
\end{aligned}
$$

In the electron energy region $250 \mathrm{keV} \leq T_{e} \leq 455 \mathrm{keV}$ and at $\lambda=-1.2750$ the obtained corrections are of order $\delta a\left(E_{e}\right) / a\left(E_{e}\right) \sim 10^{-4}, \delta A\left(E_{e}\right) / A\left(E_{e}\right)=\delta B\left(E_{e}\right) / B\left(E_{e}\right) \sim-10^{-6}, \delta K_{n}\left(E_{e}\right) / K_{n}\left(E_{e}\right) \sim \delta Q_{n}\left(E_{e}\right) / Q_{n}\left(E_{e}\right) \sim 10^{-2}$ or $\delta K_{n}\left(E_{e}\right) \sim 10^{-6}$ and $\delta Q_{n}\left(E_{e}\right) \sim-10^{-5}$. Thus, the relative corrections to all correlation coefficients except $a\left(E_{e}\right)$ and $Q_{n}\left(E_{e}\right)$, induced by the proton recoil in the Coulomb electron-proton interaction in the final state of the neutron $\beta^{-}$-decay and calculated to order $\alpha / M$, are smaller compared with corrections of order $10^{-4}$ of interactions beyond the $\mathrm{SM}$ in the experimentally used electron energy region $250 \mathrm{keV} \leq T_{e} \leq 455 \mathrm{keV}$. In turn, the correction Eq. (H-4) to correlation coefficient $a\left(E_{e}\right)$ should be taken into account for the analysis of interactions beyond the SM at the level of $10^{-4}$. It is important that the contribution of $\delta Q_{n}\left(E_{e}\right)$ to $A^{(W)}\left(E_{e}\right)$ (see Eq.(20)) is at the level of $10^{-5}$.

\section{Appendix I: Electron-proton energy distribution and proton recoil asymmetry of neutron $\beta^{-}-$decay}

The correlation coefficient $a\left(E_{e}\right)$, which analytical expression is given in Eq.(11), can be hardly used directly for the experimental determination of the correlation coefficient $a_{0}$ due to impossibility to determine experimentally a correlation between the electron and antineutrino 3-momenta. For the experimental determination of the correlation coefficient $a_{0}$ one needs to measure the correlations of charged particles, i. e. the correlations between 3-momenta of the proton and electron. For the experimental analysis of the proton recoil asymmetry we need also to have the electron-proton energy and angular distribution, including the correlations between the neutron spin and the proton 3-momentum.

After the integration over the antineutrino 3-momentum the electron-proton energy-momentum and angular distribution of the neutron $\beta^{-}$-decay takes the form

$\frac{d^{6} \lambda_{\beta_{c}^{-}}\left(E_{e}, \vec{k}_{e}, \vec{k}_{p}, \vec{\xi}_{n}\right)}{d E_{e} d k_{p} d \Omega_{p} d \Omega_{e p}}=\left(1+3 \lambda^{2}\right) \frac{G_{F}^{2}\left|V_{u d}\right|^{2}}{32 \pi^{5}}\left|\mathcal{M}_{\beta_{c}^{-}}\right|^{2}\left(1+\frac{\alpha}{\pi} g_{n}\left(E_{e}\right)\right) F\left(E_{e}, Z=1\right) \delta\left(m_{n}-E_{p}-E_{e}-E\right) \frac{m_{n}}{E_{p}} k_{e} E_{e} k_{p}^{2}$,

where $E=\left|\vec{k}_{p}+\vec{k}_{e}\right|, d \Omega_{p}=\sin \theta_{p} d \theta_{p} d \phi_{p}$ is the infinitesimal element of the solid angle of the 3-momentum of the proton relative to the polarization vector $\vec{\xi}_{n}$ of the neutron, i.e. $\vec{\xi}_{n} \cdot \vec{k}_{p}=P k_{p} \cos \theta_{p}$ with $P=\left|\vec{\xi}_{n}\right| \leq 1$. Then, $d \Omega_{e p}=\sin \theta_{e p} d \theta_{e p} d \phi_{e p}$ is the infinitesimal element of the solid angle of the correlations of the electron-proton 3momenta $\vec{k}_{e} \cdot \vec{k}_{p}=k_{e} k_{p} \cos \theta_{e p}$ and $\vec{\xi}_{n} \cdot \vec{k}_{e}=P k_{e}\left(\cos \theta_{p} \cos \theta_{e p}+\sin \theta_{p} \sin \theta_{e p} \cos \left(\phi_{p}-\phi_{e p}\right)\right)$. In the non-relativistic approximation for the proton and to next-to-leading order in the large $M$ expansion we replace $m_{n} / E_{p}$ by 


$$
\frac{m_{n}}{E_{p}} \rightarrow 1+\frac{E_{0}}{M}
$$

Then, $\left|\mathcal{M}_{\beta_{c}^{-}}\right|^{2}$, multiplied by the factor Eq.(I-2) ), is (see Eq.(A-17)

$$
\begin{aligned}
& \left(1+\frac{E_{0}}{M}\right)\left|\mathcal{M}_{\beta_{c}^{-}}\right|^{2}=1+\tilde{a}\left(E_{e}\right) \frac{\vec{k}_{e} \cdot \vec{k}}{E_{e} E}+\frac{1}{M} \frac{1}{1+3 \lambda^{2}}\left\{E_{0}-\frac{m_{e}^{2}}{E_{e}}-\left(\lambda^{2}+2(\kappa+1) \lambda\right) \frac{\vec{k} \cdot \vec{k}_{p}}{E}-\left(\lambda^{2}-2(\kappa+1) \lambda\right) \frac{\vec{k}_{e} \cdot \vec{k}_{p}}{E_{e}}\right\} \\
& +\tilde{A}\left(E_{e}\right) \frac{\vec{\xi}_{n} \cdot \vec{k}_{e}}{E_{e}}+\tilde{B}\left(E_{e}\right) \frac{\vec{\xi}_{n} \cdot \vec{k}}{E}+\frac{1}{M} \frac{1}{1+3 \lambda^{2}}\left\{-(2 \kappa+1) \lambda\left(\vec{\xi}_{n} \cdot \vec{k}_{p}\right)+\lambda \frac{\left(\vec{\xi}_{n} \cdot \vec{k}_{p}\right)\left(\vec{k}_{e} \cdot \vec{k}\right)}{E_{e} E}+\left(\lambda^{2}+(\kappa+1) \lambda+(\kappa+1)\right)\right. \\
& \left.\times \frac{\left(\vec{\xi}_{n} \cdot \vec{k}_{e}\right)\left(\vec{k} \cdot \vec{k}_{p}\right)}{E_{e} E}-\left(\lambda^{2}-(\kappa+1) \lambda+(\kappa+1)\right) \frac{\left(\vec{\xi}_{n} \cdot \vec{k}\right)\left(\vec{k}_{e} \cdot \vec{k}_{p}\right)}{E_{e} E}\right\},
\end{aligned}
$$

where $\vec{k}=-\vec{k}_{p}-\vec{k}_{e}, E=\left|\vec{k}_{p}+\vec{k}_{e}\right|$ and we have denoted

$$
\begin{aligned}
& \tilde{a}\left(E_{e}\right)=a_{0}\left(1+\frac{1}{1-\lambda^{2}} \frac{E_{0}}{M}\right)\left(1+\frac{\alpha}{\pi} f_{n}\left(E_{e}\right)\right), \\
& \tilde{A}\left(E_{e}\right)=A_{0}\left(1+\frac{1}{2(1+\lambda)} \frac{E_{0}}{M}\right)\left(1+\frac{\alpha}{\pi} f_{n}\left(E_{e}\right)\right), \\
& \tilde{B}\left(E_{e}\right)=B_{0}\left(1+\frac{1}{2(1-\lambda)} \frac{1}{M}\left(E_{0}-\frac{m_{e}^{2}}{E_{e}}\right)\right) .
\end{aligned}
$$

Substituting Eq. (I-3) into Eq. (I-1) we obtain the electron-proton energy-momentum and angular distribution of the neutron $\beta^{-}$-decay with polarized neutron and unpolarised electron and proton

$$
\begin{aligned}
& \frac{d^{6} \lambda_{\beta_{c}^{-}}\left(E_{e}, \vec{k}_{e}, \vec{k}_{p}, \vec{\xi}_{n}\right)}{d E_{e} d k_{p} d \Omega_{p} d \Omega_{e p}}=\left(1+3 \lambda^{2}\right) \frac{G_{F}^{2}\left|V_{u d}\right|^{2}}{32 \pi^{5}}\left\{1+\tilde{a}\left(E_{e}\right) \frac{\vec{k}_{e} \cdot \vec{k}}{E_{e} E}+\frac{1}{M} \frac{1}{1+3 \lambda^{2}}\left(E_{0}-\frac{m_{e}^{2}}{E_{e}}-\left(\lambda^{2}+2(\kappa+1) \lambda\right) \frac{\vec{k} \cdot \vec{k}_{p}}{E}\right.\right. \\
& \left.-\left(\lambda^{2}-2(\kappa+1) \lambda\right) \frac{\vec{k}_{e} \cdot \vec{k}_{p}}{E_{e}}\right)+\tilde{A}\left(E_{e}\right) \frac{\vec{\xi}_{n} \cdot \vec{k}_{e}}{E_{e}}+\tilde{B}\left(E_{e}\right) \frac{\vec{\xi}_{n} \cdot \vec{k}}{E}+\frac{1}{M} \frac{1}{1+3 \lambda^{2}}\left(-(2 \kappa+1) \lambda\left(\vec{\xi}_{n} \cdot \vec{k}_{p}\right)+\lambda \frac{\left(\vec{\xi}_{n} \cdot \vec{k}_{p}\right)\left(\vec{k}_{e} \cdot \vec{k}\right)}{E_{e} E}\right. \\
& \left.\left.+\left(\lambda^{2}+(\kappa+1) \lambda+(\kappa+1)\right) \frac{\left(\vec{\xi}_{n} \cdot \vec{k}_{e}\right)\left(\vec{k} \cdot \vec{k}_{p}\right)}{E_{e} E}-\left(\lambda^{2}-(\kappa+1) \lambda+(\kappa+1)\right) \frac{\left(\vec{\xi}_{n} \cdot \vec{k}\right)\left(\vec{k}_{e} \cdot \vec{k}_{p}\right)}{E_{e} E}\right)\right\} \\
& \times\left(1+\frac{\alpha}{\pi} g_{n}\left(E_{e}\right)\right) F\left(E_{e}, Z=1\right) \delta\left(m_{n}-E_{p}-E_{e}-E\right) k_{e} E_{e} k_{p}^{2},
\end{aligned}
$$

Now let us analyse the case of unpolarised neutrons. Integrating over the solid angles $d \Omega_{p}$ and $d \Omega_{e p}$ we obtain the electron-proton energy spectrum

$$
\frac{d^{2} \lambda_{\beta_{c}^{-}}\left(E_{e}, T_{p}\right)}{d E_{e} d T_{p}}=M\left(1+3 \lambda^{2}\right) \frac{G_{F}^{2}\left|V_{u d}\right|^{2}}{4 \pi^{3}} a\left(E_{e}, T_{p}\right)\left(1+\frac{\alpha}{\pi} g_{n}\left(E_{e}\right)\right) F\left(E_{e}, Z=1\right) E_{e}
$$

where $T_{p}=k_{p}^{2} / 2 M$ is the kinetic energy of the proton. The electron-proton energy distribution $a\left(E_{e}, T_{p}\right)$ takes the form

$$
a\left(E_{e}, T_{p}\right)=\zeta_{1}\left(E_{e}, T_{p}\right)+\tilde{a}\left(E_{e}\right) \zeta_{2}\left(E_{e}, T_{p}\right)
$$

The functions $\zeta_{1}\left(E_{e}, T_{p}\right)$ and $\zeta_{2}\left(E_{e}, T_{p}\right)$ are determined by the integrals over $\cos \theta_{e p}=\vec{k}_{e} \cdot \vec{k}_{p} / k_{e} k_{p}$

$$
\begin{gathered}
\zeta_{1}\left(E_{e}, T_{p}\right)=k_{e} k_{p} \int_{-1}^{+1}\left\{1+\frac{1}{M} \frac{1}{1+3 \lambda^{2}}\left[E_{0}-\frac{m_{e}^{2}}{E_{e}}+\left(\lambda^{2}+2(\kappa+1) \lambda\right) \frac{k_{p}^{2}+\vec{k}_{e} \cdot \vec{k}_{p}}{\left|\vec{k}_{p}+\vec{k}_{e}\right|}-\left(\lambda^{2}-2(\kappa+1) \lambda\right) \frac{\vec{k}_{e} \cdot \vec{k}_{p}}{E_{e}}\right]\right\} \\
\times \delta\left(m_{n}-E_{p}-E_{e}-\left|\vec{k}_{p}+\vec{k}_{e}\right|\right) d \cos \theta_{e p}
\end{gathered}
$$

and

$$
\zeta_{2}\left(E_{e}, T_{p}\right)=-k_{e} k_{p} \int_{-1}^{+1} \frac{k_{e}^{2}+\vec{k}_{e} \cdot \vec{k}_{p}}{E_{e}\left|\vec{k}_{p}+\vec{k}_{e}\right|} \delta\left(m_{n}-E_{p}-E_{e}-\left|\vec{k}_{p}+\vec{k}_{e}\right|\right) d \cos \theta_{e p}
$$


The integration over $\cos \theta_{e p}$ we perform by making a change of variables $E=\left|\vec{k}_{p}+\vec{k}_{e}\right|$. This gives

$$
\begin{aligned}
\zeta_{1}\left(E_{e}, T_{p}\right)= & \left\{E+\frac{1}{M} \frac{1}{1+3 \lambda^{2}}\left[\left(E_{0}-\frac{m_{e}^{2}}{E_{e}}\right) E+\frac{1}{2}\left(\lambda^{2}+2(\kappa+1) \lambda\right)\left(E^{2}+k_{p}^{2}-k_{e}^{2}\right)\right.\right. \\
& \left.\left.-\frac{1}{2} \frac{E}{E_{e}}\left(\lambda^{2}-2(\kappa+1) \lambda\right)\left(E^{2}-k_{p}^{2}-k_{e}^{2}\right)\right]\right\}, \\
\zeta_{2}\left(E_{e}, T_{p}\right)= & -\frac{1}{2} \frac{1}{E_{e}}\left(E^{2}-k_{p}^{2}+k_{e}^{2}\right)
\end{aligned}
$$

at $E=m_{n}-E_{p}-E_{e}$. The energy region of the definition of the distribution $a\left(E_{e}, T_{p}\right)$ is given by [106]

$$
\begin{aligned}
0 & \leq T_{p} \leq\left(T_{p}\right)_{\max }=\frac{\left(m_{n}-m_{p}\right)^{2}-m_{e}^{2}}{2 m_{n}}=\frac{E_{0}^{2}-m_{e}^{2}}{2 M}=0.751 \mathrm{keV}, \\
\left(E_{e}\right)_{\min } & \leq E_{e} \leq\left(E_{e}\right)_{\max },
\end{aligned}
$$

where $\left(E_{e}\right)_{\min / \max }$ are equal to

$$
\left(E_{e}\right)_{\min / \max }=\frac{\left(m_{n}-E_{p} \mp k_{p}\right)^{2}+m_{e}^{2}}{2\left(m_{n}-E_{p} \mp k_{p}\right)}=\frac{\left(E_{0} \mp \sqrt{2 M T_{p}}\right)^{2}+m_{e}^{2}}{2\left(E_{0} \mp \sqrt{2 M T_{p}}\right)}+\frac{1}{2}\left(1-\frac{m_{e}^{2}}{\left(E_{0} \mp \sqrt{2 M T_{p}}\right)^{2}}\right)\left(\left(T_{p}\right)_{\max }-T_{p}\right) .
$$

Here we have kept the next-to-leading terms in the large $M$ expansion and used the relation

$$
m_{n}-m_{p}=E_{0}+\frac{E_{0}^{2}-m_{e}^{2}}{2 M}=E_{0}+\left(T_{p}\right)_{\max }
$$

To next-to-leading order in the large $M$ expansion the variable $E$ is defined by

$$
E=m_{n}-E_{p}-E_{e}=E_{0}-E_{e}+\left(\left(T_{p}\right)_{\max }-T_{p}\right) .
$$

The functions $\zeta_{1}\left(E_{e}, T_{p}\right)$ and $\zeta_{2}\left(E_{e}, T_{p}\right)$, determined to order $1 / M$, are equal to

$$
\begin{aligned}
& \zeta_{1}\left(E_{e}, T_{p}\right)=\left(E_{0}-E_{e}\right)+\left(\left(T_{p}\right)_{\max }-T_{p}\right)+\frac{1}{M} \frac{1}{1+3 \lambda^{2}}\left[\left(E_{0}-\frac{m_{e}^{2}}{E_{e}}\right)\left(E_{0}-E_{e}\right)\right. \\
& -\frac{1}{2} \frac{E_{0}}{E_{e}}\left(\left(E_{0}-E_{e}\right)^{2}+E_{e}^{2}-m_{e}^{2}-2 M T_{p}\right)+\frac{1}{2}\left(\lambda^{2}+2(\kappa+1) \lambda\right)\left(\left(E_{0}-E_{e}\right)^{2}-E_{e}^{2}+m_{e}^{2}-2 M T_{p}\right) \\
& \left.-\frac{1}{2}\left(\lambda^{2}-2(\kappa+1) \lambda\right) \frac{E_{0}-E_{e}}{E_{e}}\left(\left(E_{0}-E_{e}\right)^{2}-E_{e}^{2}+m_{e}^{2}-2 M T_{p}\right)\right], \\
& \zeta_{2}\left(E_{e}, T_{p}\right)=-\frac{1}{2} \frac{1}{E_{e}}\left(\left(E_{0}-E_{e}\right)^{2}+E_{e}^{2}-m_{e}^{2}-2 M T_{p}+2\left(E_{0}-E_{e}\right)\left(\left(T_{p}\right)_{\max }-T_{p}\right)\right) .
\end{aligned}
$$

Integrating the electron-proton energy spectrum Eq.(I-6) over the electron energy we obtain the proton-energy spectrum

$$
\frac{d \lambda_{\beta_{c}^{-}}\left(T_{p}\right)}{d T_{p}}=M\left(1+3 \lambda^{2}\right) \frac{G_{F}^{2}\left|V_{u d}\right|^{2}}{4 \pi^{3}} a\left(T_{p}\right)
$$

where $a\left(T_{p}\right)$ is defined by

$$
a\left(T_{p}\right)=g_{1}\left(T_{p}\right)+a_{0}\left(1+\frac{1}{1-\lambda^{2}} \frac{E_{0}}{M}\right) g_{2}\left(T_{p}\right)
$$

with the functions $g_{1}\left(T_{p}\right)$ and $g_{2}\left(T_{p}\right)$, given by the integrals

$$
\begin{aligned}
& g_{1}\left(T_{p}\right)=\int_{\left(E_{e}\right)_{\min }}^{\left(E_{e}\right)_{\max }} \zeta_{1}\left(E_{e}, T_{p}\right)\left(1+\frac{\alpha}{\pi} g_{n}\left(E_{e}\right)\right) F\left(E_{e}, Z=1\right) E_{e} d E_{e} \\
& g_{2}\left(T_{p}\right)=\int_{\left(E_{e}\right)_{\min }}^{\left(E_{e}\right)_{\max }} \zeta_{2}\left(E_{e}, T_{p}\right)\left(1+\frac{\alpha}{\pi} g_{n}\left(E_{e}\right)+\frac{\alpha}{\pi} f_{n}\left(E_{e}\right)\right) F\left(E_{e}, Z=1\right) E_{e} d E_{e} .
\end{aligned}
$$


For the calculation of the proton recoil asymmetry $C$, first, we have to integrate over the azimuthal angles. After the integration we arrive at the expression

$$
\begin{aligned}
& \frac{d^{4} \lambda_{\beta_{c}^{-}}\left(E_{e}, k_{p}, \theta_{p}, \theta_{e p}, P\right)}{d E_{e} d k_{p} d \cos \theta_{p} d \cos \theta_{e p}}=\left(1+3 \lambda^{2}\right) \frac{G_{F}^{2}\left|V_{u d}\right|^{2}}{8 \pi^{3}}\left\{1-\tilde{a}\left(E_{e}\right) \frac{k_{e}^{2}+k_{e} k_{p} \cos \theta_{e p}}{E_{e} E}+\frac{1}{M} \frac{1}{1+3 \lambda^{2}}\right. \\
& \times\left(E_{0}-\frac{m_{e}^{2}}{E_{e}}+\left(\lambda^{2}+2(\kappa+1) \lambda\right) \frac{k_{p}^{2}+k_{e} k_{p} \cos \theta_{e p}}{E}-\left(\lambda^{2}-2(\kappa+1) \lambda\right) \frac{k_{e} k_{p} \cos \theta_{e p}}{E_{e}}\right) \\
& +P \cos \theta_{p}\left[\tilde{A}\left(E_{e}\right) \frac{k_{e} \cos \theta_{e p}}{E_{e}}-\tilde{B}\left(E_{e}\right) \frac{k_{e} \cos \theta_{e p}+k_{p}}{E}+\frac{1}{M} \frac{1}{1+3 \lambda^{2}}\left(\left(-(2 \kappa+1) \lambda-\lambda \frac{k_{e}^{2}+k_{e} k_{p} \cos \theta_{e p}}{E_{e} E}\right) k_{p}\right.\right. \\
& \left.\left.\left.-2(\kappa+1) \lambda \frac{k_{e} k_{p} \cos \theta_{e p}}{E_{e} E}\left(k_{p}+k_{e} \cos \theta_{e p}\right)\right)\right]\right\}\left(1+\frac{\alpha}{\pi} g_{n}\left(E_{e}\right)\right) F\left(E_{e}, Z=1\right) \delta\left(m_{n}-E_{p}-E_{e}-E\right) k_{e} E_{e} k_{p}^{2} .
\end{aligned}
$$

Having integrated over $\cos \theta_{e p}$ we obtain the following result

$$
\begin{aligned}
& \frac{d^{3} \lambda_{\beta_{c}^{-}}\left(E_{e}, k_{p}, \theta_{p}, P\right)}{d E_{e} d k_{p} d \cos \theta_{p}}=\left(1+3 \lambda^{2}\right) \frac{G_{F}^{2}\left|V_{u d}\right|^{2}}{16 \pi^{3}}\left\{2\left(E_{0}-E_{e}\right) E_{e} k_{p}-\tilde{a}\left(E_{e}\right)\left(\left(E_{0}-E_{e}\right)^{2}-k_{p}^{2}+k_{e}^{2}\right) k_{p}+\frac{1}{M} \frac{1}{1+3 \lambda^{2}}\right. \\
& \times\left(2\left(E_{0} E_{e}-m_{e}^{2}\right)\left(E_{0}-E_{e}\right) k_{p}+\left(1+3 \lambda^{2}\right) E_{e}\left(E_{0}^{2}-m_{e}^{2}-k_{p}^{2}\right) k_{p}-\left(1-\lambda^{2}\right)\left(E_{0}-E_{e}\right)\left(E_{0}^{2}-m_{e}^{2}-k_{p}^{2}\right) k_{p}\right. \\
& \left.+\left(\lambda^{2}+2(\kappa+1) \lambda\right) E_{e}\left(\left(E_{0}-E_{e}\right)^{2}+k_{p}^{2}-k_{e}^{2}\right) k_{p}-\left(\lambda^{2}-2(\kappa+1) \lambda\right)\left(E_{0}-E_{e}\right)\left(\left(E_{0}-E_{e}\right)^{2}-k_{p}^{2}-k_{e}^{2}\right) k_{p}\right) \\
& +P \cos \theta_{p}\left[\tilde{A}\left(E_{e}\right)\left(E_{0}-E_{e}\right)\left(\left(E_{0}-E_{e}\right)^{2}-k_{p}^{2}-k_{e}^{2}\right)-\tilde{B}\left(E_{e}\right) E_{e}\left(\left(E_{0}-E_{e}\right)^{2}+k_{p}^{2}-k_{e}^{2}\right)+\frac{1}{M} \frac{1}{1+3 \lambda^{2}}\right. \\
& \times\left(-(2 \kappa+1) \lambda 2\left(E_{0}-E_{e}\right) E_{e} k_{p}^{2}-\lambda\left(\left(E_{0}-E_{e}\right)^{2}-k_{p}^{2}+k_{e}^{2}\right) k_{p}^{2}-(\kappa+1) \lambda\left(\left(\left(E_{0}-E_{e}\right)^{2}-k_{e}^{2}\right)^{2}-k_{p}^{4}\right)\right. \\
& \left.\left.\left.-\lambda(1+\lambda)\left(3\left(E_{0}-E_{e}\right)^{2}-k_{p}^{2}-k_{e}^{2}\right)\left(E_{0}^{2}-m_{e}^{2}-k_{p}^{2}\right)+\lambda(1-\lambda) 2\left(E_{0}-E_{e}\right) E_{e}\left(E_{0}^{2}-m_{e}^{2}-k_{p}^{2}\right)\right)\right]\right\} \\
& \times\left(1+\frac{\alpha}{\pi} g_{n}\left(E_{e}\right)\right) F\left(E_{e}, Z=1\right),
\end{aligned}
$$

where we have kept the terms of order $1 / M$ and the radiative corrections of order $\alpha / \pi$. The integration over the electron energy $E_{e}$ and the proton momentum $k_{p}$ gives one

$$
\begin{aligned}
& \frac{d \lambda_{\beta_{c}^{-}}\left(\theta_{p}, P\right)}{d \cos \theta_{p}}=\left(1+3 \lambda^{2}\right) \frac{G_{F}^{2}\left|V_{u d}\right|^{2}}{16 \pi^{3}}\left\{X_{1}+\frac{\alpha}{\pi} X_{2}+\frac{1}{M} \frac{1}{1+3 \lambda^{2}}\left[X_{3}+\left(1+3 \lambda^{2}\right)\left(X_{4}+Y_{1}\right)-\left(1-\lambda^{2}\right)\left(X_{5}+Y_{2}\right)\right.\right. \\
& \left.+\left(\lambda^{2}+2(\kappa+1) \lambda\right) X_{6}-\left(\lambda^{2}-2(\kappa+1) \lambda\right) X_{7}\right]+P \cos \theta_{p}\left[-\left(A_{0}+B_{0}\right) X_{8}+A_{0} X_{9}+A_{0} \frac{\alpha}{\pi} X_{10}-B_{0} \frac{\alpha}{\pi} X_{11}\right. \\
& \left.\left.+\frac{1}{M} \frac{1}{1+3 \lambda^{2}}\left(\lambda X_{12}-(\kappa+1) \lambda X_{13}-(2 \kappa+1) \lambda X_{14}-\lambda(1+\lambda)\left(X_{15}+Y_{3}\right)+\lambda(1-\lambda)\left(X_{16}+Y_{4}\right)\right)\right]\right\} .
\end{aligned}
$$

The factors $X_{j}$ for $j=1, \ldots, 16$ are calculated for the limits of the integration over $E_{e}$ and $k_{p}$ equal to

$$
\begin{gathered}
0 \leq k_{p} \leq\left(k_{p}\right)_{\max }=\sqrt{E_{0}^{2}-m_{e}^{2}}, \\
\left(E_{e}\right)_{\min }=\frac{\left(E_{0}-k_{p}\right)^{2}+m_{e}^{2}}{2\left(E_{0}-k_{p}\right)} \leq E_{e} \leq\left(E_{e}\right)_{\max }=\frac{\left(E_{0}+k_{p}\right)^{2}+m_{e}^{2}}{2\left(E_{0}+k_{p}\right)} .
\end{gathered}
$$

The factors $Y_{j}$ for $j=1,2,3,4$ are defined by the contributions of the $1 / M$ corrections to the limits of the integration over $E_{e}$ and $k_{p}$. The $1 / M$ corrections to the limits of the integration over $E_{e}$ are given in Eq. (I-12). The limits of the integration over $k_{p}$ have no $1 / M$ corrections

$$
\left(k_{p}\right)_{\max }=\sqrt{\frac{m_{p}}{m_{n}}\left(\left(m_{n}-m_{p}\right)^{2}-m_{e}^{2}\right)}=\sqrt{E_{0}^{2}-m_{e}^{2}} .
$$


The account for the $1 / M$ corrections to the limits of the integration over $E_{e}$ can be carried out by the formula

$$
\begin{aligned}
& \int_{0}^{\left(k_{p}\right)_{\max }} \int_{\left(E_{e}\right)_{\min }+\Delta E_{-}\left(k_{p}\right)}^{\left(E_{e}\right)_{\max }+\Delta E_{+}\left(k_{p}\right)} \Phi\left(E_{e}, k_{p}\right) d E_{e} d k_{p}=\int_{0}^{\left(k_{p}\right)_{\max }} \int_{\left(E_{e}\right)_{\min }\left(k_{p}\right)}^{\left(E_{e}\right)_{\max }\left(k_{p}\right)} \Phi\left(E_{e}, k_{p}\right) d E_{e} d k_{p} \\
& +\int_{0}^{\left(k_{p}\right)_{\max }}\left[\Phi\left(\left(E_{e}\right)_{\max }, k_{p}\right) \Delta E_{+}\left(k_{p}\right)-\Phi\left(\left(E_{e}\right)_{\min }, k_{p}\right) \Delta E_{-}\left(k_{p}\right)\right] d k_{p},
\end{aligned}
$$

where $\Delta E_{ \pm}\left(k_{p}\right)$ are equal to

$$
\Delta E_{ \pm}\left(k_{p}\right)=\frac{1}{2}\left(1-\frac{m_{e}^{2}}{\left(E_{0} \pm \sqrt{2 M T_{p}}\right)^{2}}\right)\left(\left(T_{p}\right)_{\max }-T_{p}\right) .
$$

The results of the integration are

$$
\begin{aligned}
& X_{1}=\int_{0}^{\left(k_{p}\right)_{\max }} \int_{\left(E_{e}\right)_{\min }}^{\left(E_{e}\right)_{\max }} 2\left(E_{0}-E_{e}\right) E_{e} k_{p} F\left(E_{e}, Z=1\right) d E_{e} d k_{p}=0.235040 \mathrm{MeV}^{5}, \\
& X_{2}=\int_{0}^{\left(k_{p}\right)_{\max }} \int_{\left(E_{e}\right)_{\min }}^{\left(E_{e}\right)_{\max }} 2\left(E_{0}-E_{e}\right) E_{e} k_{p} g_{n}\left(E_{e}\right) F\left(E_{e}, Z=1\right) d E_{e} d k_{p}=3.932201 \mathrm{MeV}^{5} \text {, } \\
& X_{3}=\int_{0}^{\left(k_{p}\right)_{\max }} \int_{\left(E_{e}\right)_{\min }}^{\left(E_{e}\right)_{\max }} 2\left(E_{0} E_{e}-m_{e}^{2}\right)\left(E_{0}-E_{e}\right) k_{p} F\left(E_{e}, Z=1\right) d E_{e} d k_{p}=0.225097 \mathrm{MeV}^{6} \text {, } \\
& X_{4}=\int_{0}^{\left(k_{p}\right)_{\max }} \int_{\left(E_{e}\right)_{\min }}^{\left(E_{e}\right)_{\max }} E_{e}\left(E_{0}^{2}-m_{e}^{2}-k_{p}^{2}\right) k_{p} F\left(E_{e}, Z=1\right) d E_{e} d k_{p}=0.190889 \mathrm{MeV}^{6}, \\
& X_{5}=\int_{0}^{\left(k_{p}\right)_{\max }} \int_{\left(E_{e}\right)_{\min }}^{\left(E_{e}\right)_{\max }}\left(E_{0}-E_{e}\right)\left(E_{0}^{2}-m_{e}^{2}-k_{p}^{2}\right) k_{p} F\left(E_{e}, Z=1\right) d E_{e} d k_{p}=0.112935 \mathrm{MeV}^{6}, \\
& X_{6}=\int_{0}^{\left(k_{p}\right)_{\max }} \int_{\left(E_{e}\right)_{\min }}^{\left(E_{e}\right)_{\max }} E_{e}\left(\left(E_{0}-E_{e}\right)^{2}+k_{p}^{2}-k_{e}^{2}\right) k_{p} F\left(E_{e}, Z=1\right) d E_{e} d k_{p}=0.112935 \mathrm{MeV}^{6}, \\
& X_{7}=\int_{0}^{\left(k_{p}\right)_{\max }} \int_{\left(E_{e}\right)_{\min }}^{\left(E_{e}\right)_{\max }}\left(E_{0}-E_{e}\right)\left(\left(E_{0}-E_{e}\right)^{2}-k_{p}^{2}-k_{e}^{2}\right) k_{p} F\left(E_{e}, Z=1\right) d E_{e} d k_{p}=-0.112162 \mathrm{MeV}^{6}, \\
& X_{8}=\int_{0}^{\left(k_{p}\right)_{\max }} \int_{\left(E_{e}\right)_{\min }}^{\left(E_{e}\right)_{\max }} E_{e}\left(\left(E_{0}-E_{e}\right)^{2}+k_{p}^{2}-k_{e}^{2}\right) F\left(E_{e}, Z=1\right) d E_{e} d k_{p}=0.129699 \mathrm{MeV}^{5}, \\
& X_{9}=\int_{0}^{\left(k_{p}\right)_{\max }} \int_{\left(E_{e}\right)_{\min }}^{\left(E_{e}\right)_{\max }}\left[\left(E_{0}-E_{e}\right)\left(\left(E_{0}-E_{e}\right)^{2}-k_{p}^{2}-k_{e}^{2}\right)+E_{e}\left(\left(E_{0}-E_{e}\right)^{2}+k_{p}^{2}-k_{e}^{2}\right)\right] F\left(E_{e}, Z=1\right) d E_{e} d k_{p}= \\
& =0.001059 \mathrm{MeV}^{5} \text {, } \\
& X_{10}=\int_{0}^{\left(k_{p}\right)_{\max }} \int_{\left(E_{e}\right)_{\min }}^{\left(E_{e}\right)_{\max }}\left(E_{0}-E_{e}\right)\left(\left(E_{0}-E_{e}\right)^{2}-k_{p}^{2}-k_{e}^{2}\right)\left(g_{n}\left(E_{e}\right)+f_{n}\left(E_{e}\right)\right) F\left(E_{e}, Z=1\right) d E_{e} d k_{p}= \\
& =-2.157085 \mathrm{MeV}^{5} \text {, } \\
& X_{11}=\int_{0}^{\left(k_{p}\right)_{\max }} \int_{\left(E_{e}\right)_{\min }}^{\left(E_{e}\right)_{\max }} E_{e}\left(\left(E_{0}-E_{e}\right)^{2}+k_{p}^{2}-k_{e}^{2}\right) g_{n}\left(E_{e}\right) F\left(E_{e}, Z=1\right) d E_{e} d k_{p}=2.244146 \mathrm{MeV}^{5} \\
& X_{12}=\int_{0}^{\left(k_{p}\right)_{\max }} \int_{\left(E_{e}\right)_{\min }}^{\left(E_{e}\right)_{\max }}\left[E_{0} E_{e}\left(\left(E_{0}-E_{e}\right)^{2}+k_{p}^{2}-k_{e}^{2}\right)-E_{0}\left(E_{0}-E_{e}\right)\left(\left(E_{0}-E_{e}\right)^{2}-k_{p}^{2}-k_{e}^{2}\right)\right. \\
& \left.-\left(\left(E_{0}-E_{e}\right)^{2}-k_{p}^{2}+k_{e}^{2}\right) k_{p}^{2}\right] F\left(E_{e}, Z=1\right) d E_{e} d k_{p}=0.353804 \mathrm{MeV}^{6} \text {, } \\
& X_{13}=\int_{0}^{\left(k_{p}\right)_{\max }} \int_{\left(E_{e}\right)_{\min }}^{\left(E_{e}\right)_{\max }}\left(\left(\left(E_{0}-E_{e}\right)^{2}-k_{e}^{2}\right)^{2}-k_{p}^{4}\right) F\left(E_{e}, Z=1\right) d E_{e} d k_{p}=-0.119181 \mathrm{MeV}^{6} \text {, } \\
& X_{14}=\int_{0}^{\left(k_{p}\right)_{\max }} \int_{\left(E_{e}\right)_{\min }}^{\left(E_{e}\right)_{\max }} 2\left(E_{0}-E_{e}\right) E_{e} k_{p}^{2} F\left(E_{e}, Z=1\right) d E_{e} d k_{p}=0.186815 \mathrm{MeV}^{6} \\
& X_{15}=\int_{0}^{\left(k_{p}\right)_{\max }} \int_{\left(E_{e}\right)_{\min }}^{\left(E_{e}\right)_{\max }}\left(3\left(E_{0}-E_{e}\right)^{2}-k_{p}^{2}-k_{e}^{2}\right)\left(E_{0}^{2}-m_{e}^{2}-k_{p}^{2}\right) F\left(E_{e}, Z=1\right) d E_{e} d k_{p}=-0.002707 \mathrm{MeV}^{6},
\end{aligned}
$$




$$
\begin{aligned}
& X_{16}=\int_{0}^{\left(k_{p}\right)_{\max }} \int_{\left(E_{e}\right)_{\min }}^{\left(E_{e}\right)_{\max }} 2\left(E_{0}-E_{e}\right) E_{e}\left(E_{0}^{2}-m_{e}^{2}-k_{p}^{2}\right) F\left(E_{e}, Z=1\right) d E_{e} d k_{p}=0.291289 \mathrm{MeV}^{6}, \\
& X_{17}=\int_{0}^{\left(k_{p}\right)_{\max }} \int_{\left(E_{e}\right)_{\min }}^{\left(E_{e}\right)_{\max }} \frac{m_{e}}{E_{e}}\left(E_{0}-E_{e}\right)\left(\left(E_{0}-E_{e}\right)^{2}-k_{p}^{2}-k_{e}^{2}\right) F\left(E_{e}, Z=1\right) d E_{e} d k_{p}=-0.076306 \mathrm{MeV}^{5}, \\
& X_{18}=\int_{0}^{\left(k_{p}\right)_{\max }} \int_{\left(E_{e}\right)_{\min }}^{\left(E_{e}\right)_{\max }} m_{e}\left(\left(E_{0}-E_{e}\right)^{2}+k_{p}^{2}-k_{e}^{2}\right) F\left(E_{e}, Z=1\right) d E_{e} d k_{p}=0.093560 \mathrm{MeV}^{5} .
\end{aligned}
$$

The factors $X_{17}$ and $X_{18}$ are related to contributions of interactions beyond the SM (see section IX). The factors $Y_{j}$ for $j=1,2,3,4$ are given by

$$
\begin{aligned}
& Y_{1}=\frac{1}{2} \int_{0}^{\left(k_{p}\right)_{\max }}\left[\left(E_{0}-\left(E_{e}\right)_{\max }\right)\left(E_{e}\right)_{\max }\left(1-\frac{m_{e}^{2}}{\left(E_{0}+k_{p}\right)^{2}}\right) F\left(\left(E_{e}\right)_{\max }, Z=1\right)\right. \\
& \left.-\left(E_{0}-\left(E_{e}\right)_{\min }\right)\left(E_{e}\right)_{\min }\left(1-\frac{m_{e}^{2}}{\left(E_{0}-k_{p}\right)^{2}}\right) F\left(\left(E_{e}\right)_{\min }, Z=1\right)\right]\left(E_{0}^{2}-m_{e}^{2}-k_{p}^{2}\right) k_{p} d k_{p}=0.077953 \mathrm{MeV}^{6}, \\
& Y_{2}=\frac{1}{4} \int_{0}^{\left(k_{p}\right)_{\max }}\left[\left(\left(E_{0}-\left(E_{e}\right)_{\max }\right)^{2}-k_{p}^{2}+\left(E_{e}\right)_{\max }^{2}-m_{e}^{2}\right)\left(1-\frac{m_{e}^{2}}{\left(E_{0}+k_{p}\right)^{2}}\right)\right. \\
& \times F\left(\left(E_{e}\right)_{\max }, Z=1\right)-\left(\left(E_{0}-\left(E_{e}\right)_{\min }\right)^{2}-k_{p}^{2}+\left(E_{e}\right)_{\min }^{2}-m_{e}^{2}\right)\left(1-\frac{m_{e}^{2}}{\left(E_{0}-k_{p}\right)^{2}}\right) \\
& \left.\times F\left(\left(E_{e}\right)_{\min }, Z=1\right)\right]\left(E_{0}^{2}-m_{e}^{2}-k_{p}^{2}\right) k_{p} d k_{p}=-0.000772 \mathrm{MeV}^{6}, \\
& Y_{3}=\frac{1}{2} \int_{0}^{\left(k_{p}\right)_{\max }}\left[\left(E_{0}-\left(E_{e}\right)_{\max }\right)\left(\left(E_{0}-\left(E_{e}\right)_{\max }\right)^{2}-k_{p}^{2}-\left(E_{e}\right)_{\max }^{2}+m_{e}^{2}\right)\left(1-\frac{m_{e}^{2}}{\left(E_{0}+k_{p}\right)^{2}}\right)\right. \\
& \times F\left(\left(E_{e}\right)_{\max }, Z=1\right)-\left(E_{0}-\left(E_{e}\right)_{\min }\right)\left(\left(E_{0}-\left(E_{e}\right)_{\min }\right)^{2}-k_{p}^{2}-\left(E_{e}\right)_{\min }^{2}+m_{e}^{2}\right)\left(1-\frac{m_{e}^{2}}{\left(E_{0}-k_{p}\right)^{2}}\right) \\
& \left.\times F\left(\left(E_{e}\right)_{\min }, Z=1\right)\right]\left(E_{0}^{2}-m_{e}^{2}-k_{p}^{2}\right) d k_{p}=-0.204003 \mathrm{MeV}^{6}, \\
& Y_{4}=\frac{1}{2} \int_{0}^{\left(k_{p}\right)_{\max }}\left[\left(E_{e}\right)_{\max }\left(\left(E_{0}-\left(E_{e}\right)_{\max }\right)^{2}+k_{p}^{2}-\left(E_{e}\right)_{\max }^{2}+m_{e}^{2}\right)\left(1-\frac{m_{e}^{2}}{\left(E_{0}+k_{p}\right)^{2}}\right)\right. \\
& \times F\left(\left(E_{e}\right)_{\max }, Z=1\right)-\left(E_{e}\right)_{\min }\left(\left(E_{0}-\left(E_{e}\right)_{\min }\right)^{2}+k_{p}^{2}-\left(E_{e}\right)_{\min }^{2}+m_{e}^{2}\right)\left(1-\frac{m_{e}^{2}}{\left(E_{0}-k_{p}\right)^{2}}\right) \\
& \left.\times F\left(\left(E_{e}\right)_{\min }, Z=1\right)\right]\left(E_{0}^{2}-m_{e}^{2}-k_{p}^{2}\right) d k_{p}=-0.082343 \mathrm{MeV}^{6} .
\end{aligned}
$$

The correctness of our calculation of the electron-proton energy distribution $a\left(E_{e}, T_{p}\right)$ we may verify by calculating the lifetime of the neutron. Having integrated Eq.(I-21) over $\cos \theta_{p}$ we obtain the rate of the neutron $\beta^{-}$-decay

$$
\begin{aligned}
\left(\lambda_{\beta_{c}^{-}}\right)_{\mathrm{SM}} & =\left(1+3 \lambda^{2}\right) \frac{G_{F}^{2}\left|V_{u d}\right|^{2}}{8 \pi^{3}}\left\{X_{1}+\frac{\alpha}{\pi} X_{2}+\frac{1}{M} \frac{1}{1+3 \lambda^{2}}\left[X_{3}+\left(1+3 \lambda^{2}\right)\left(X_{4}+Y_{1}\right)-\left(1-\lambda^{2}\right)\left(X_{5}+Y_{2}\right)\right.\right. \\
& \left.\left.+\left(\lambda^{2}+2(\kappa+1) \lambda\right) X_{6}-\left(\lambda^{2}-2(\kappa+1) \lambda\right) X_{7}\right]\right\}
\end{aligned}
$$

related to the lifetime as $\left(\lambda_{\beta_{c}^{-}}\right)_{\mathrm{SM}}^{-1}=\left(\tau_{n}\right)_{\mathrm{SM}}$. The numerical factors $X_{j}$ and $Y_{k}$ for $j=1, \ldots, 7$ and $k=1,2$ define the lifetime of the neutron with the account for the radiative $\left(X_{2} \neq 0\right)$ and the $1 / M\left(X_{j} \neq 0\right.$ for $j=3, \ldots, 7$ and $Y_{k} \neq 0$ for $k=1,2$ ) corrections.

Let us consider three possibilities: 1) $X_{j}=0$ for $j=2, \ldots, 7$ and $Y_{k}=0$ for $k=1,2$ (i.e. without radiative and $1 / M$ corrections), 2) $X_{k} \neq 0$ for $k=1,2$ and $X_{j}=0$ for $j=3, \ldots, 7$ and $Y_{k}=0$ for $k=1,2$ (i.e. with radiative but without $1 / M$ corrections) and 3$) X_{k} \neq 0$ for $k=1, \ldots, 7$ and $Y_{k} \neq 0$ for $k=1,2$ (with radiative and $1 / M$ corrections). For these cases we obtain 1) $\left.\left(\tau_{n}\right)_{\mathrm{SM}}=915.3 \mathrm{~s}, 2\right)\left(\tau_{n}\right)_{\mathrm{SM}}=881.0 \mathrm{~s}$ and 3$)\left(\tau_{n}\right)_{\mathrm{SM}}=879.6 \mathrm{~s}$, respectively. These results agree well with the lifetimes of the neutron, adduced in Table I (see section $\mathrm{X}$ ).

The correlation of the neutron spin and the proton 3-momentum is described by the angular distribution 72 ]

$$
4 \pi \frac{d W\left(\theta_{p}\right)}{d \Omega_{p}}=1+2 P C \cos \theta_{p}
$$


where $P$ is a neutron polarization. The correlation coefficient $C$ is defined by

$$
\begin{aligned}
& C=-\frac{1}{2} \frac{X_{8}}{X_{1}}\left(A_{0}+B_{0}\right)+\frac{1}{2} \frac{X_{9}}{X_{1}} A_{0}+\frac{\alpha}{\pi} \frac{1}{2} \frac{X_{10}}{X_{1}} A_{0}-\frac{\alpha}{\pi} \frac{1}{2} \frac{X_{11}}{X_{1}} B_{0}+\frac{1}{M} \frac{1}{1+3 \lambda^{2}}\left(\lambda \frac{1}{2} \frac{X_{12}}{X_{1}}-(\kappa+1) \lambda \frac{1}{2} \frac{X_{13}}{X_{1}}\right. \\
& \left.-(2 \kappa+1) \lambda \frac{1}{2} \frac{X_{14}}{X_{1}}-\lambda(1+\lambda) \frac{1}{2} \frac{X_{15}+Y_{3}}{X_{1}}+\lambda(1-\lambda) \frac{1}{2} \frac{X_{16}+Y_{4}}{X_{1}}\right)+\left(A_{0}+B_{0}\right) \frac{X_{8}}{X_{1}}\left\{\frac{\alpha}{\pi} \frac{1}{2} \frac{X_{2}}{X_{1}}+\frac{1}{M} \frac{1}{1+3 \lambda^{2}}\right. \\
& \left.\times\left(\frac{1}{2} \frac{X_{3}}{X_{1}}+\left(1+3 \lambda^{2}\right) \frac{1}{2} \frac{X_{4}+Y_{1}}{X_{1}}-\left(1-\lambda^{2}\right) \frac{1}{2} \frac{X_{5}+Y_{2}}{X_{1}}+\left(\lambda^{2}+2(\kappa+1) \lambda\right) \frac{1}{2} \frac{X_{6}}{X_{1}}-\left(\lambda^{2}-2(\kappa+1) \lambda\right) \frac{1}{2} \frac{X_{7}}{X_{1}}\right)\right\},
\end{aligned}
$$

where the factor $x_{C}=X_{8} / 2 X_{1}=0.27591$ agrees well with the factor $x_{C}=0.27594$, calculated by Glück [73]. The apperance of the term $A_{0} X_{9} / 2 X_{1}$ is related to the deviation of the Fermi function $F\left(E_{e}, Z=1\right)$ from unity.

The contributions of interactions beyond the SM (see Appendix G) changes the electron-proton energy distribution $a\left(E_{e}, T_{p}\right)$ as follows

$$
\begin{aligned}
& a_{\mathrm{eff}}\left(E_{e}, T_{p}\right)=\left(1+\frac{1}{1+3 \lambda^{2}}\left(\operatorname{Re}\left(\delta C_{V}-\delta \bar{C}_{V}\right)-3 \lambda \operatorname{Re}\left(\delta C_{A}-\delta \bar{C}_{A}\right)\right)+b_{F} \frac{m_{e}}{E_{e}}\right) \zeta_{1}\left(E_{e}, T_{p}\right) \\
& +\left[a_{0}+\frac{1}{1+3 \lambda^{2}}\left(\operatorname{Re}\left(\delta C_{V}-\delta \bar{C}_{V}\right)+\lambda \operatorname{Re}\left(\delta C_{A}-\delta \bar{C}_{A}\right)\right)\right]\left(1+\frac{1}{1-\lambda^{2}} \frac{E_{0}}{M}\right)\left(1+\frac{\alpha}{\pi} f_{n}\left(E_{e}\right)\right) \zeta_{2}\left(E_{e}, T_{p}\right)
\end{aligned}
$$

Multiplying $a\left(E_{e}, T_{p}\right)$ by the lifetime of the neutron $\tau_{n}$ (see Eq. (G-15) we obtain

$$
\begin{aligned}
& \tau_{n} a_{\mathrm{eff}}\left(E_{e}, T_{p}\right)=\left(\tau_{n}\right)_{\mathrm{SM}}\left\{\left(1+b_{F} \frac{m_{e}}{E_{e}}-b_{F}\left\langle\frac{m_{e}}{E_{e}}\right\rangle_{\mathrm{SM}}\right) \zeta_{1}\left(E_{e}, T_{p}\right)+\left[a_{0}+\frac{1}{1+3 \lambda^{2}}\left(\operatorname{Re}\left(\delta C_{V}-\delta \bar{C}_{V}\right)+\lambda\right.\right.\right. \\
& \left.\left.\times \operatorname{Re}\left(\delta C_{A}-\delta \bar{C}_{A}\right)\right)-a_{0}\left(\frac{1}{1+3 \lambda^{2}}\left(\operatorname{Re}\left(\delta C_{V}-\delta \bar{C}_{V}\right)-3 \lambda \operatorname{Re}\left(\delta C_{A}-\delta \bar{C}_{A}\right)\right)+b_{F}\left\langle\frac{m_{e}}{E_{e}}\right\rangle_{\mathrm{SM}}\right)\right]\left(1+\frac{1}{1-\lambda^{2}} \frac{E_{0}}{M}\right) \\
& \left.\times\left(1+\frac{\alpha}{\pi} f_{n}\left(E_{e}\right)\right) \zeta_{2}\left(E_{e}, T_{p}\right)\right\} .
\end{aligned}
$$

This allows to reduce the analysis of contributions of vector and axial-vector interactions beyond the SM to the electron-proton energy distribution to the analysis of these contributions to the axial coupling constant $\lambda$ (see section $\mathrm{VI}$ and section $[\mathrm{IX}$ ). Contributions of interactions beyond the SM to the proton recoil asymmetry $C$ are given in section IX

The radiative corrections to the electron-proton energy distribution $a\left(E_{e}, T_{p}\right)$ Eq. (I-7) or the proton-energy spectrum $a\left(T_{p}\right)$ Eq. (I-17) and the proton recoil asymmetry $C$ Eq. (I-30) have been calculated at the neglect the contributions of the proton-photon correlations in the proton recoil energy and angular distribution of the radiative $\beta^{-}-$decay of the neutron. As has been pointed out by Glück 77], the contributions of the radiative $\beta^{-}-$decay of the neutron to the proton recoil energy and angular distribution, caused by the proton-photon correlations, demand a detailed analysis. For the aim of a consistent calculation of the contributions of the nucleus-photon and hadron-photon correlations in the radiative nuclear and hadronic $\beta$-decays Glück has used the Monte Carlo simulation method. The calculation of the proton-photon correlations has been recently performed in [78]. There has been shown that the contributions of the proton-photon correlations to the lifetime of the neutron $\tau_{n}$ and the electron-proton energy distribution $a\left(E_{e}, T_{p}\right)$ (or the proton-energy spectrum $a\left(T_{p}\right)$ ) are of order $10^{-5}$ and can be neglected with respect to the contributions of order $10^{-4}$ of interactions beyond the SM. Such a neglect of the contributions of the proton-photon correlations confirms the correctness of the use the functions $(\alpha / \pi) g_{n}\left(E_{e}\right)$ and $(\alpha / \pi) f_{n}\left(E_{e}\right)$ for the description of the radiative corrections in the lifetime of the neutron and the electron-proton energy distribution (or the proton-energy spectrum), respectively. In turn, the contributions of the proton-photon correlations to the proton recoil angular distribution and the proton recoil asymmetry $C$ are of order $10^{-4}$. Hence they should be taken into account for the correct determination of contributions of order $10^{-4}$ beyond the SM. The contributions of these corrections changes the proton recoil asymmetry $C$ as follows [78]

$$
\begin{aligned}
& C=-\left(x_{C}+\frac{\alpha}{\pi} x_{\text {eff }}\right)\left(A_{0}+B_{0}\right)+\frac{1}{2} \frac{X_{9}}{X_{1}} A_{0}+\frac{1}{M} \frac{1}{1+3 \lambda^{2}}\left(\lambda \frac{1}{2} \frac{X_{12}}{X_{1}}-(\kappa+1) \lambda \frac{1}{2} \frac{X_{13}}{X_{1}}-(2 \kappa+1) \lambda \frac{1}{2} \frac{X_{14}}{X_{1}}\right. \\
& \left.-\lambda(1+\lambda) \frac{1}{2} \frac{X_{15}+Y_{3}}{X_{1}}+\lambda(1-\lambda) \frac{1}{2} \frac{X_{16}+Y_{4}}{X_{1}}\right)+\left(A_{0}+B_{0}\right) \frac{X_{8}}{X_{1}}\left\{\frac{\alpha}{\pi} \frac{1}{2} \frac{X_{2}}{X_{1}}+\frac{1}{M} \frac{1}{1+3 \lambda^{2}}\left(\frac{1}{2} \frac{X_{3}}{X_{1}}+\left(1+3 \lambda^{2}\right)\right.\right. \\
& \left.\left.\times \frac{1}{2} \frac{X_{4}+Y_{1}}{X_{1}}-\left(1-\lambda^{2}\right) \frac{1}{2} \frac{X_{5}+Y_{2}}{X_{1}}+\left(\lambda^{2}+2(\kappa+1) \lambda\right) \frac{1}{2} \frac{X_{6}}{X_{1}}-\left(\lambda^{2}-2(\kappa+1) \lambda\right) \frac{1}{2} \frac{X_{7}}{X_{1}}\right)\right\},
\end{aligned}
$$

where $x_{\mathrm{eff}}=X_{\mathrm{eff}} / 2 X_{1}=4.712120$ 78. One may see that the contributions of the proton-photon correlations make the radiative corrections to the proton recoil asymmetry $C$ symmetric with respect to a change $A_{0} \longleftrightarrow B_{0}$ as well as 
the main term $C_{0}=-x_{C}\left(A_{0}+B_{0}\right)$.

[1] H. Abele, Progr. Part. Nucl. Phys. 60, 1 (2008).

[2] J. S. Nico, J. Phys. G: Nucl. Part. Phys. 36, 104001 (2009).

[3] M. J. Ramsey-Musolf and S. Su, Phys. Rep. 456, 1 (2008) and references therein.

[4] J. D. Jackson, S. B. Treiman, and H. W. Wyld Jr., Phys. Rev. 106, 517 (1957); Nucl. Phys. 4, 206 (1957).

[5] M. E. Ebel and G. Feldman, Nucl. Phys. 4, 213 (1957).

[6] P. Herczeg, Progr. Part. Nucl. Phys. 46, 413 (2001).

[7] N. Severijns, M. Beck, and O. Naviliat-Cuncic, Rev. Mod. Phys. 78, 991 (2006).

[8] There is also the pseudoscalar lepton-baryon weak coupling constant, the contribution of which can be neglected with respect to the contributions of order $10^{-4}$.

[9] J. Beringer et al. (Particle Data Group), Phys. Rev. D $\underline{86}, 010001$ (2012).

[10] M. Faber et al., Phys. Rev. C 80, 035503 (2009).

[11] V. De Alfaro et al., in Currents in hadron physics, North-Holland Publishing Co., Amsterdam-London, 1973.

[12] A. P. Serebrov et al., Phys. Rev. C 78, 035505 (2008).

[13] R. E. Behrends, R. J. Finkelstein, and A. Sirlin, Phys. Rev. 101, 866 (1956).

[14] S. M. Berman, Phys. Rev. 112, 267 (1958).

[15] T. Kinoshita and A. Sirlin, Phys. Rev. 113, 1652 (1959).

[16] S. M. Berman and A. Sirlin, Ann. Phys. (N.Y.) 20, 20 (1962).

[17] G. Källén, Nucl. Phys. B 1, 225 (1967).

[18] A. Sirlin, Phys. Rev. 164, 1767 (1967).

[19] E. S. Abers, D. A. Dicus, R. E. Norton, and H. R. Queen, Phys. Rev. 167, 1461 (1968).

[20] R. T. Shann, Nuovo Cimento A 5, 591 (1971).

[21] A. Sirlin, Nucl. Phys. B 71, 29 (1974).

[22] W. J. Marciano and A. Sirlin, Nucl. Phys. B 88, 86 (1975).

[23] A. Sirlin, Nucl. Phys. B 100, 291 (1975).

[24] A. Sirlin, Rev. Mod. Phys. 50, 573 (1978); Rev. Mod. Phys. 50, 905 (1978).

[25] A. García and M. Maya, Phys. Rev. D 17, 1376 (1978).

[26] A. Sirlin, Nucl. Phys. B 196, 83 (1982).

[27] W. J. Marciano and A. Sirlin, Phys. Rev. Lett. 56, 22 (1986).

[28] A. Czarnecki, W. J. Marciano, and A. Sirlin, Phys. Rev. D 70, 093006 (2004).

[29] W. J. Marciano and A. Sirlin, Phys. Rev. Lett. 96, 032002 (2006).

[30] A. Czarnecki, W. J. Marciano, and A. Sirlin, Phys. Rev. Lett. 99, 032003 (2007).

[31] S. Ando et al., Phys. Lett. B 595, 250 (2004).

[32] V. Gudkov, G. I. Greene, and J. R. Calarco, Phys. Rev. C 73, 035501 (2006).

[33] B. R. Holstein, Rev. Mod. Phys. 46, 789 (1974).

[34] D. H. Wilkinson, Nucl. Phys. A 377, 474 (1982).

[35] C. Itzykson and J.-B. Zuber, in Quantum Field Theory, McGraw-Hill Inc., New York, 1980.

[36] T. Kinoshita, J. Math. Phys. 3, 650 (1962); T. D. Lee and M. Nauenberg, Phys. Rev. 133, 1549 (1964).

[37] D. Mund, PhD. thesis, University of Heidelberg, 2006.

[38] M. Schumann et al., Phys. Rev. Lett. 99, 191803 (2007).

[39] M. Schumann, PhD. thesis, University of Heidelberg, 2007, www.ub.uni-heidelberg.de/archiv/7357.

[40] J. Byrne et al., J. Phys. G: Nucl. Part. Phys. 28, 1325 (2002).

[41] C. Stratowa, R. Dobrozemsky, and P. Weinzierl, Phys. Rev. D 18, 3970 (1978).

[42] M. Schumann et al., Phys. Rev. Lett. 100, 151801 (2008).

[43] T. Bhattacharya et al., Phys. Rev. D 85, 054512 (2012).

[44] V. Cirigliano, J. Jenkins, and M. Gonzáles-Alonso, Nucl. Phys. B 830, 95 (2010); V. Cirigliano, M. Gonzáles-Alonso, and M. L. Graesser, J. High Energy Phys. 02, 046 (2013).

[45] M. Gonzáles-Alonso, (private communication).

[46] J. S. Nico et al., Nature, 444, 1059 (2006).

[47] R. L. Cooper et al., Phys. Rev. C 81, 035503 (2010).

[48] R. L. Cooper, PhD Thesis, University of Michigan, 2008.

[49] Yu. V. Gaponov and R. U. Khafisov, Phys. At. Nucl. 59, 1213 (1996) (Yad. Fiz. 59, 1270 (1996)); Phys. Lett. B 379, 7 (1996); Nucl. Instr. Meth. in Phys. Res., A 440, 557 (2000).

[50] V. Bernard et al., Phys. Lett. B 593, 105 (2004), Erratum Phys. Lett. B 599, 348 (2004).

[51] J. F. Donoghue, E. Golowich, and B. R. Holstein, in Dynamics of the Standard Model, Cambridge University Press, 1992.

[52] J. C. Hardy and I. S. Towner, Phys. Rev. C 79, 055502 (2009); I. S. Towner and J. C. Hardy, Rep. Prog. Phys. 73, 046301 (2010).

[53] R. Pohl et al., Nature 466, 213 (2010).

[54] C. G. Callan and S. B. Treiman, Phys. Rev. 162, 1494 (1967). 
[55] H. H. Chen, Phys. Rev. 185, 2003 (1969).

[56] J. C. Brodine, Phys. Rev. D 1, 100 (1970); Phys. Rev. D 2, 2090 (1970).

[57] B. R. Holstein, Phys. Rev. C 5, 1529 (1972); Phys. Rev. C 28, 342 (1983).

[58] P. Herczeg and I. B. Khriplovich, Phys. Rev. D 56, 80 (1997).

[59] J. D. Jackson, S. B. Treiman, and H. W. Wyld, Jr., Z. Phys. 150, 640 (1958).

[60] E. K. Konopinski, in The theory of beta radioactivity, Oxford at the Clarendon Press, Oxford, 1966.

[61] S. I. Ando, J. A. McGovern, and T. Sato, Phys. Lett. B 677, 109 (2009).

[62] D. Dubbers et al., Nucl. Instr. and Meth. Phys. Res. A 596, 238 (2008).

[63] In comparison with [34], we have replaced $\lambda \rightarrow-\lambda$ and included the factor $2(\lambda-\kappa-1)$ into the definition of the coefficients $A_{j}^{(\mathrm{W})}$ for $j=1,2,3$.

[64] F. Glück, I. Joó, and J. Last, Nucl. Phys. A 593, 125 (1995).

[65] F. Glück, Phys. Lett. B 436, 25 (1998).

[66] A. P. Serebrov et al., Zh. Exsp. Teor. Fiz. 113, 1963 (1998), J. Exp. Theor.Phys. 86, 1074 (1998).

[67] I. A. Kuznetzov et al., Phys. Rev. Lett. 75, 794 (1995).

[68] M. Kreuz et al., Phys. Lett. B 619, 263 (2005).

[69] G. Konrad, PhD Thesis, Johannes Gutenberg - Universität Mainz, 2011 and references therein.

[70] X. Wang, G. Konrad, and H. Abele, Nucl. Instr. and Meth. Phys. Res. A 701, 254 (2013); arXiv:1209.6595 [physics.insdet].

[71] F. Glück, Phys. Rev. D 47, 2840 (1993).

[72] S. B. Treiman, Phys. Rev. 110, 448 (1958).

[73] F. Glück, Phys. Lett. B 376, 25 (1996).

[74] S. Gardner and C. Zhang, Phys. Rev. Lett. 86, 5666 (2001).

[75] S. K. L. Sjue, Phys. Rev. C 72, 045501 (2005).

[76] V. Gudkov, Phys. Rev. C 77, 045502 (2008).

[77] F. Glück, Computer Physics Communications 101, 223 (1997).

[78] A. N. Ivanov, R. Höllwieser, N. I. Troitskaya, and M. Wellenzohn, Phys. Rev. D 88, 065026 (2013).

[79] A. Pichlmaier et al., Phys. Lett. B 693, 221 (2010).

[80] S. S. Arzumanov et al., JETPL 95, 224 (2012).

[81] A. P. Serebrov and A. K. Fomin, Phys. Rev. C 82, 035501 (2010); A. P. Serebrov, A. K. Fomin, arXiv: 1104.4238 [nucl-ex].

[82] D. Dubbers and M. G. Schmidt, Rev. Mod. Phys. 83, 1111 (2011).

[83] H. P. Mumm et al., Phys. Rev. Lett. 107, 102301 (2011).

[84] D. Mund et al., Phys. Rev. Lett. 110, 172502 (2013); arXiv: 1204.0013 [hep-ex].

[85] G. Konrad et al., in Proceedings of the 5th International BEYOND 2010 Conference, Cape Town, South Africa (2010) (World Scientific, 2011), pp. 660-672, arXiv: 1007.3027 [nucl-ex].

[86] S. Paul, Nucl. Instrum. Meth. Phys. Res. A 611, 157 (2009); arXiv: 0902.0169v2 [hep-ex], http://www.e18.physik.tu-muenchen.de/research

[87] J. Liu et al., Phys. Rev. Lett. 105, 181803 (2010); Phys. Rev. Lett. 105, 219903 (2010) (Erratum).

[88] M. P. Mendenhall et al., Phys. Rev. C 87, 032501 (2013).

[89] P. Liaud et al., Nucl. Phys. A 612, 53 (1997).

[90] B. G. Erozolimsky et at., Phys. Lett. B 412, 240 (1997).

[91] P. Bopp et al., Phys. Rev. Lett. 56, 919 (1986).

[92] A. Sirlin, AIP Conf. Proc. 698, 385 (2004).

[93] A. Sirlin, Phys. Rev. D 35, 3423 (1987); A. Sirlin and R. Zucchini, Phys. Rev. Lett. 57, 1994 (1986).

[94] A. Kurylov, M. J. Ramsey-Musolf, and P. Vogel, Phys. Rev. C 67, 035502 (2003); Phys. Rev. C 65, 055501 (2002).

[95] A. N. Ivanov et al., arXiv:1306.1995 [hep-ph].

[96] P. Vogel, Phys. Rev. D 29, 1918 (1984).

[97] S. A. Fayans, Yad. Fiz. 42, 929 (1985) [Sov. J. Nucl. Phys. 42, 590 (1985).]

[98] M. Fukugita and T. Kubota, Acta Phys. Pol. B 35, 1687 (2004); Acta Phys. Pol. B 37, 2741(E) (2006).

[99] U. Raha, F. Myhrer, and K. Kudobera, Phys. Rev. C 85, 045502 (2012); Erratum: Phys. Rev. C 86, 039903 (2012).

[100] A. Sirlin, Phys. Rev. D 84, 014021 (2011).

[101] A. Sirlin and A. Ferroglia, Rev. Mod. Phys. 85, 263 (2013).

[102] In Eq.(3) of Ref.[47] the sum of the momenta $\left(\vec{p}_{e}+2 \vec{k}\right)$ should be replaced by $\left(2 \vec{p}_{e}+\vec{k}\right)$. After such a replacement Eq. (B-10) for the unpolarised neutron coincides fully with Eq. (3) of Ref. 47].

[103] Handbook of Mathematical Functions with Formulas, Graphs, and Mathematical Tables, ed. by M. Abramowitz and I. A. Stegun, Tenth Printing with corrections, National Bureau of Standards Applied Mathematics Series • 55, p.1004, 1972; K. Mitchell, Phil. Mag. 40, 351 (1949); E. S. Ginsberg and D. Zaborowski, Communications of the ACM, 18, 200 (1975).

[104] J. M. Jauch and F. Rohrlich, in The Theory of Photons and Electrons, The Relativistic Quantum Field Theory of Charged Particles with Spin One-Half, Second expended edition, Springer-Verlag, Berlin, 1976.

[105] With respect to the definition of the coupling constants in the original paper [6] we have extracted the common factor $G_{F} V_{u d} / \sqrt{2}$ and replaced $a_{L L}^{h}$ and $a_{L R}^{h}$ by $a_{L L}^{h} \rightarrow a_{L L}^{h}+(1-\lambda) / 2$ and $a_{L R}^{h} \rightarrow a_{L R}^{h}+(1+\lambda) / 2$, respectively, where the coupling constants $a_{L L}^{h}$ and $a_{L R}^{h}$ describe deviations from the coupling constants of the SM.

[106] O. Nachtmann, Z. Phys. 215, 505 (1968). 